University of Tennessee Health Science Center UTHSC Digital Commons

\title{
Development and Evaluation of Nano-scale Systems for Targeted Delivery to Treat Liver Fibrosis
}

\author{
Ningning Yang \\ University of Tennessee Health Science Center
}

Follow this and additional works at: https://dc.uthsc.edu/dissertations

Part of the Pharmaceutics and Drug Design Commons

\section{Recommended Citation}

Yang, Ningning , "Development and Evaluation of Nano-scale Systems for Targeted Delivery to Treat Liver Fibrosis" (2011). Theses and Dissertations (ETD). Paper 310. http://dx.doi.org/10.21007/ etd.cghs.2011.0364.

This Dissertation is brought to you for free and open access by the College of Graduate Health Sciences at UTHSC Digital Commons. It has been accepted for inclusion in Theses and Dissertations (ETD) by an authorized administrator of UTHSC Digital Commons. For more information, please contact jwelch30@uthsc.edu. 


\title{
Development and Evaluation of Nano-scale Systems for Targeted Delivery to Treat Liver Fibrosis
}

\begin{abstract}
Excessive deposition of ECM is the common characteristic of liver fibrosis. During hepatic fibrosis, various inflammatory cytokines are released and trigger the activation of quiescent HSCs. The activated HSCs play the major role in producing extra amount collagen. It becomes very crucial to focus on HSCs to find out therapeutics, such as inhibiting collagen synthesis, inhibiting activation to myofibroblasts, or controlling inflammation.

To control excessive collagen synthesis, one triplex forming oligonucleotides (TFO), was systemically administrated to prevent type I collagen mRNA transcription. To enhance circulation time and targeted delivery efficiency, TFO was conjugated to M6P-HPMA and showed efficient targeted delivery to HSCs. Two week short term in vivo i.v. administration also showed the therapeutic effects on liver fibrosis by M6P-HPMA-TFO.
\end{abstract}

Transforming growth factor $\beta 1$ (TGF- $\beta 1$ ) acts as the initial factor for liver fibrosis. TGF- $\beta 1$ gene was demonstrated to be interfered by siRNA in a sequence and dose dependent mode in HSC-T6 cell line. Later, GFAP promoter driven HSC-specific pri-miRNA mimic and pri-miRNA cluster mimic showed HSC-specific TGF- $\beta 1$ gene silencing to avoid nonspecific inhibition of TGF- $\beta 1$ expression in other cells and organs.

The novel LPA antagonist, PTP, can interact with LPA receptor on the surface of cells to inhibit the proliferation, which is also one consequence of HSCs activation. However, the low aqueous solubility affects its in vivo application. Therefore, poly(ethylene glycol)-b-poly(carbonate-co-lactide) copolymers were used to make micellar formulation to enhance solubility. PEG-PCCL micelles were applied to increase the aqueous solubility of PTP. In vivo administration of PTP loaded PEG-PCcL showed therapeutic effects on fibrosis in common bile duct ligated mice.

\section{Document Type}

Dissertation

Degree Name

Doctor of Philosophy (PhD)

Program

Pharmaceutical Sciences

Research Advisor

Ram I. Mahato, Ph.D.

\section{Keywords}

gene therapy, HSC, liver fibrosis, nano, targeted delivery

\section{Subject Categories}

Medicine and Health Sciences | Pharmaceutics and Drug Design | Pharmacy and Pharmaceutical Sciences 


\title{
DEVELOPMENT AND EVALUATION OF NANO-SCALE SYSTEMS FOR TARGETED DELIVERY TO TREAT LIVER FIBROSIS
}

\author{
A Dissertation \\ Presented for \\ The Graduate Studies Council \\ The University of Tennessee \\ Health Science Center
}

In Partial Fulfillment

Of the Requirements for the Degree

Doctor of Philosophy

From The University of Tennessee

By

Ningning Yang

December 2011 
Portions of Chapter 2 (C) 2010 by CRC Press.

Portions of Chapters 3, 4, and 5 (C) 2009 by American Chemical Society.

Portions of Chapter 6 (c) 2011 by Springer.

All other material (C) 2011 by Ningning Yang.

All rights reserved. 


\section{DEDICATION}

This dissertation is dedicated to my parents, Jun Yang and Konghua Liang, my husband, Dr. Manrong Jiang,

my daughter, Grace Ziwei Jiang and my son, Benjamin Zilai Jiang, for their endless love and continuous support. 


\section{ACKNOWLEDGEMENTS}

I could never have reached the heights or explored the depths without the help, support, guidance and efforts of a lot of people. The most important of all, I would like to express my deepest and sincere appreciation to my mentor Dr. Ram I. Mahato for his guidance, understanding, patience, and most importantly, his friendship during my graduate studies at University of Tennessee Health Science Center. His mentorship was paramount in providing a well rounded experience consistent my long-term career goals. I would also like to thank my committee members, Dr. Duane D. Miller, Dr. Sarka Beranova, Dr. Wei Li and Dr. Xin Zhang, for their priceless suggestions, guidance and assistance over the years.

I would also like to thank Drs. Zhaoyang Ye, Guofeng Cheng and Ravikiran Panakanti for their valuable guidance and help early on in my PhD program. I am very grateful for the friendship of all of the members in the research group, Dr. Xiangxu Jia, Dr. Kun Cheng, Dr. Lin Zhu, Feng Li, Michael Danquah, Hao Wu and Dr. Wenli Lu.

I would like to thank the University of Tennessee Health Science Center for providing me with the opportunity to pursue graduate education. I would like to thank my parents and my brothers.

Finally, and most importantly, I would like to thank my husband Dr. Manrong Jiang. His support, encouragement, quiet patience and unwavering love were undeniably the bedrock upon which the past five years of my life have been built. 


\begin{abstract}
Excessive deposition of ECM is the common characteristic of liver fibrosis. During hepatic fibrosis, various inflammatory cytokines are released and trigger the activation of quiescent HSCs. The activated HSCs play the major role in producing extra amount collagen. It becomes very crucial to focus on HSCs to find out therapeutics, such as inhibiting collagen synthesis, inhibiting activation to myofibroblasts, or controlling inflammation.

To control excessive collagen synthesis, one triplex forming oligonucleotides (TFO), was systemically administrated to prevent type I collagen mRNA transcription. To enhance circulation time and targeted delivery efficiency, TFO was conjugated to M6P-HPMA and showed efficient targeted delivery to HSCs. Two week short term in vivo i.v. administration also showed the therapeutic effects on liver fibrosis by M6P-HPMA-TFO.

Transforming growth factor $\beta 1$ (TGF- $\beta 1$ ) acts as the initial factor for liver fibrosis. TGF- $\beta 1$ gene was demonstrated to be interfered by siRNA in a sequence and dose dependent mode in HSC-T6 cell line. Later, GFAP promoter driven HSC-specific pri-miRNA mimic and pri-miRNA cluster mimic showed HSC-specific TGF- $\beta 1$ gene silencing to avoid nonspecific inhibition of TGF- $\beta 1$ expression in other cells and organs.

The novel LPA antagonist, PTP, can interact with LPA receptor on the surface of cells to inhibit the proliferation, which is also one consequence of HSCs activation. However, the low aqueous solubility affects its in vivo application. Therefore, poly(ethylene glycol)-b-poly(carbonate-co-lactide) copolymers were used to make micellar formulation to enhance solubility. PEG-PCcL micelles were applied to increase the aqueous solubility of PTP. In vivo administration of PTP loaded PEG-PCcL showed therapeutic effects on fibrosis in common bile duct ligated mice.
\end{abstract}




\section{TABLE OF CONTENTS}

CHAPTER 1. INTRODUCTION

1.1. Pathogenesis of Liver Fibrosis and Hepatic Stellate Cells (HSCs)

1.1.1. Pathogenesis of Liver Fibrosis ........................................................... 1

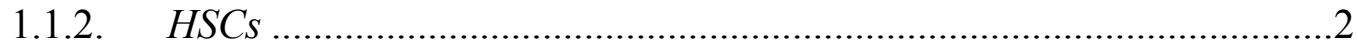

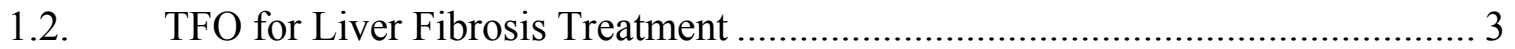

1.3. RNA Interference on TGF- $\beta 1$ to Treat Hepatic Fibrosis ................................... 5

1.4. LPA Antagonist for Liver Fibrosis Therapeutic .......................................... 6

\section{CHAPTER 2. DELIVERY AND TARGETING OF OLIGONUCLEOTIDES}

AND SIRNA ............................................................................................

2.1. Single Stranded Oligonucleotides ............................................................ 8

2.1.1. Antisense Oligodeoxyribonucleotides ...............................................8

2.1.2. Triplex Forming Oligonucleotides....................................................8

2.1.3. Immunomodulatory Oligonucleotides ............................................10

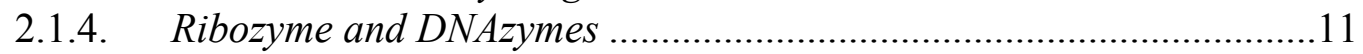

2.1.5. $\quad$ Nucleic Acid Aptamer ...................................................................... 11

2.2. Double Stranded Therapeutic Oligonucleotides ........................................... 12

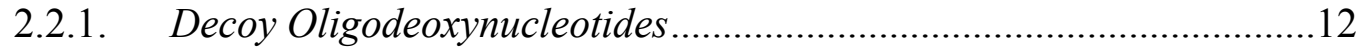

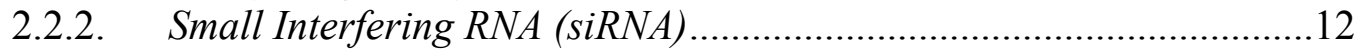

2.3. Barriers to Oligonucleotide Based Therapeutics .......................................... 13

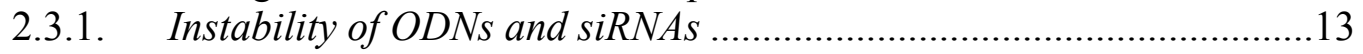

2.3.2. Non-specific Binding and Toxicity ................................................16

2.3.3. Physiological Barriers ................................................................... 17

2.4. Synthetic Carriers for Nucleic Acid Delivery............................................... 20

2.4.1. Complex Formation ..................................................................20

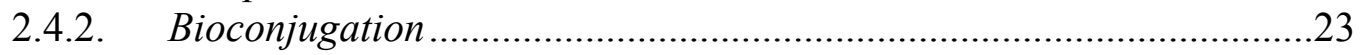

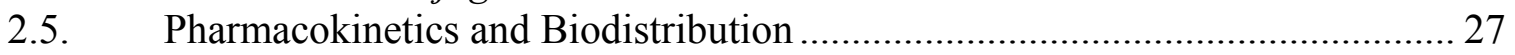

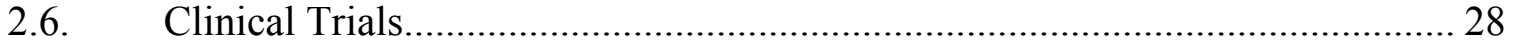

CHAPTER 3. HPMA POLYMER-BASED SITE-SPECIFIC DELIVERY OF OLIGONUCLEOTIDES TO HEPATIC STELLATE CELLS...........31

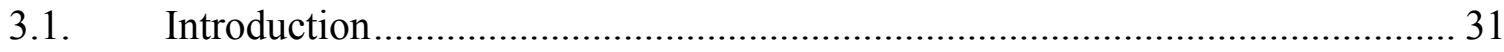

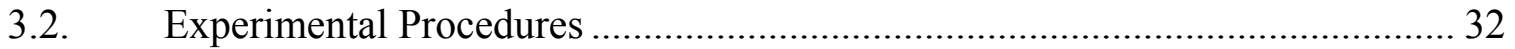

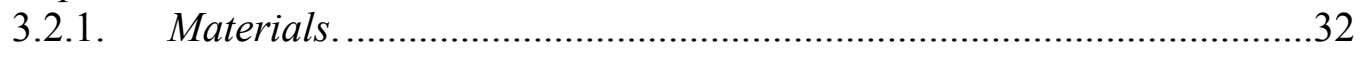

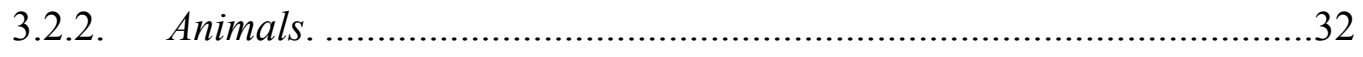

3.2.3. Synthesis of p-Isothiocyanatophenyl-6-phospho- $\alpha$-D-mannopyranoside.................32

3.2.4. Synthesis of M6P-GFLG-HPMA-GFLG-TFO Conjugate ...................33

3.2.5. In Vitro Characterization...............................................................33

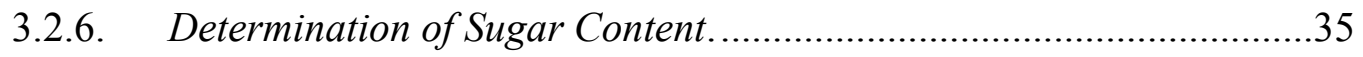

3.2.7. In Vitro Enzymatic Dissociation and Triplex Formation of

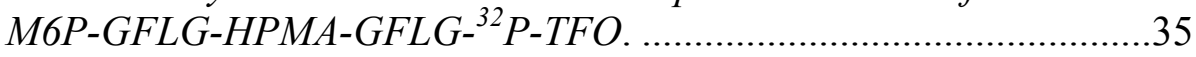

3.2.8. Transfection of TFO and M6P-GFLG-HPMA-GFLG-TFO .................35 
3.2.9. Biodistribution of M6P-GFLG-HPMA-GFLG- ${ }^{32} P-T F O$..........................

3.2.10. Determination of Pharmacokinetic Profiles.....................................36

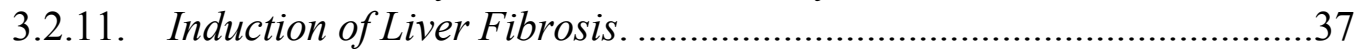

3.2.12. Competition in Hepatic Uptake of M6P-BSA- ${ }^{33} P-T F O$........................... 37

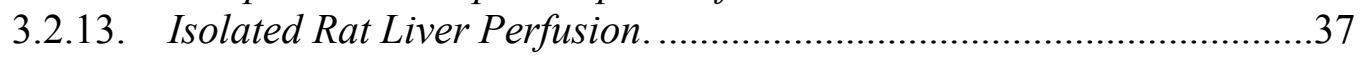

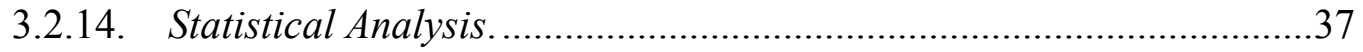

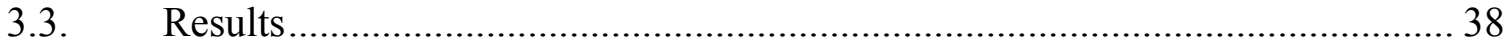

3.3.1. Synthesis and In Vitro Characterization of M6P-GFLG-HPMA-GFLG- ${ }^{32}$ P-TFO. .........................................38

3.3.2. In Vitro Enzymatic Dissociation and Triplex Formation of

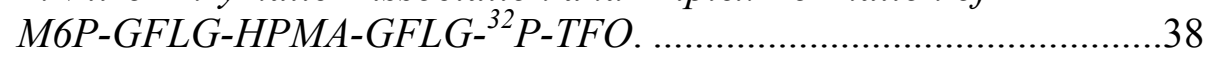

3.3.3. Inhibition of Collagen Type I Gene Expression by M6P-GFLG-HPMA-GFLG-TFO ..............................................43

3.3.4. Biodistribution of M6P-GFLG-HPMA-GFLG-TFO.........................43

3.3.5. Effect of Fibrosis on Biodistribution.................................................49

3.3.6. Competition in Hepatic Uptake. ........................................................49

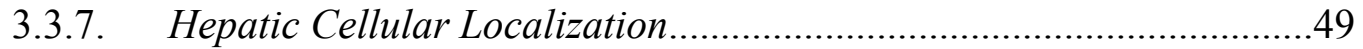

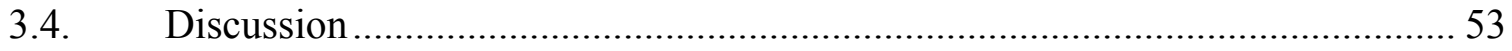

\section{CHAPTER 4. TREATMENT OF LIVER FIBROSIS AFTER SYSTEMIC} ADMINISTRATION OF M6P-HPMA-TFO ....................................55

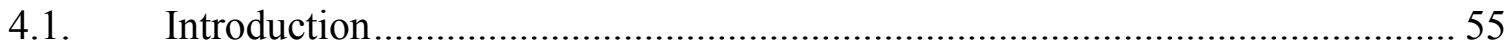

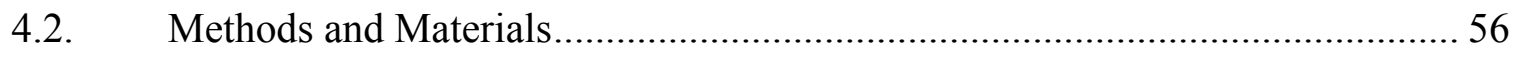

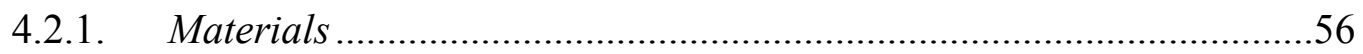

4.2.2. Synthesis and In Vitro Characterization of M6P-HPMA-TFO

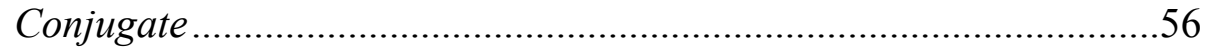

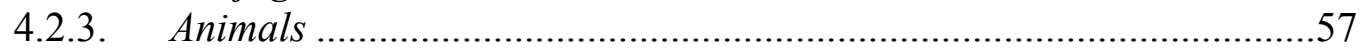

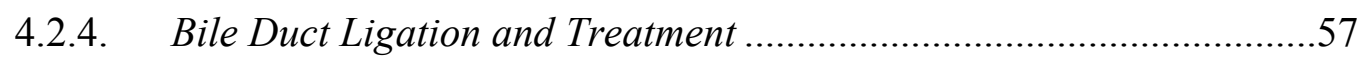

4.2.5. Standard Serum Parameters ..........................................................57

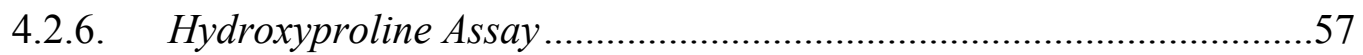

4.2.7. ELISA for Tumor Necrosis Factor $\alpha$.............................................58

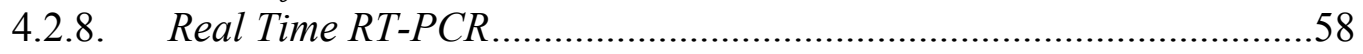

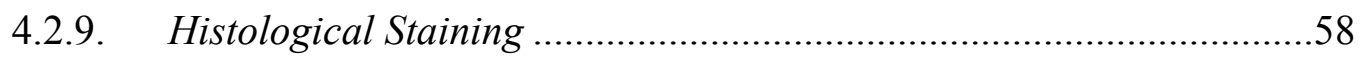

4.2.10. Immunofluorescent Staining .....................................................58

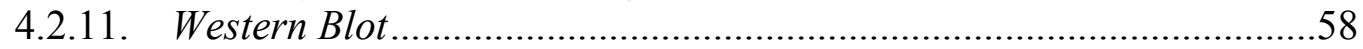

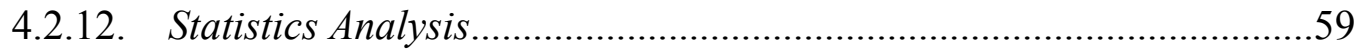

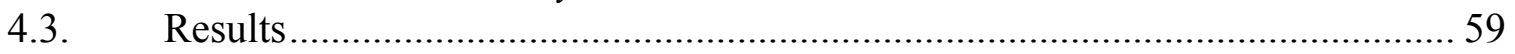

4.3.1. In Vitro Characterization of M6P-HPMA-TFO. ................................59

4.3.2. M6P-HPMA-TFO Inhibits Collagen and Attenuates Liver

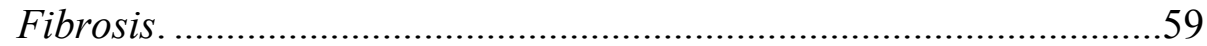

4.3.3. M6P-HPMA-TFO Inhibits Profibrogens .......................................62

4.3.4. Inhibition of Collagen Intrinsic Degradation ...................................62

4.3.5. Inhibition on Myofibroblast Activation .............................................62

4.3.6. Effects on Serum Fibrotic and Pro-inflammatory Markers .................65

4.3.7. Inhibition on Neutrophil Infiltration .............................................65

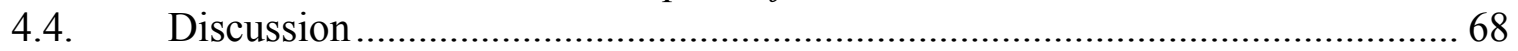




\section{CHAPTER 5. TGF-B1 GENE SILENCING FOR TREATING LIVER}

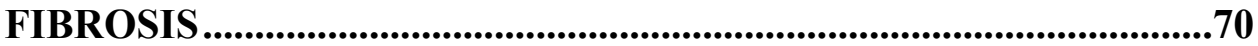

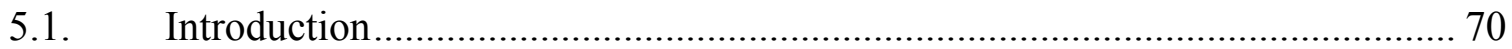

5.2. Materials and Methods.............................................................................. 72

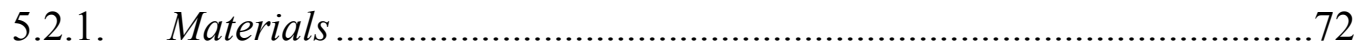

5.2.2. $\quad$ siRNA Design and Synthesis ..........................................................72

5.2.3. Design and Construction of shRNA Expression Plasmids....................72

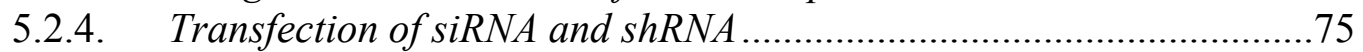

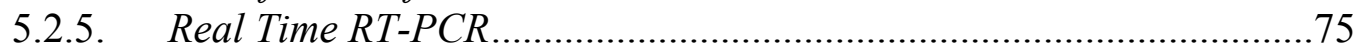

5.2.6. Western Blot Assay .........................................................................77

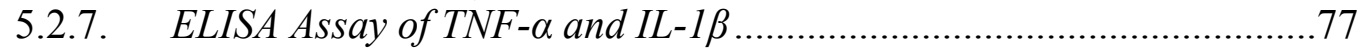

5.2.8. Persistence of TGF- $\beta 1$ Gene Silencing .............................................78

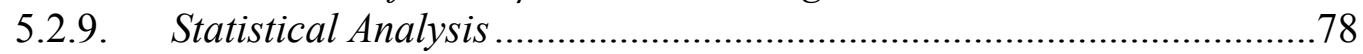

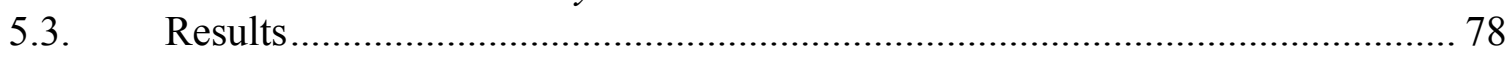

5.3.1. Effect of siRNA Sequences and Dose on TGF- $\beta 1$ Gene

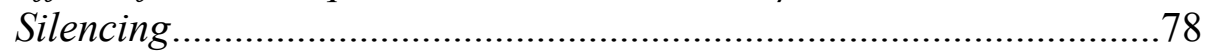

5.3.2. Effect of TGF- $\beta 1$ Gene Silencing on TIMP-1 Expression .....................81

5.3.3. Effect of siRNA on Type $\alpha(I)$ Collagen and $\alpha-S M A$ Expression ...........81

5.3.4. Construction of shRNA Expression Vector ............................................81

5.3.5. Effect of shRNA Expression on TGF- $\beta 1$ Silencing ...............................81

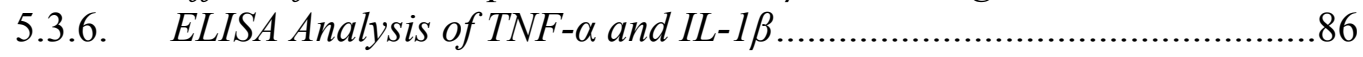

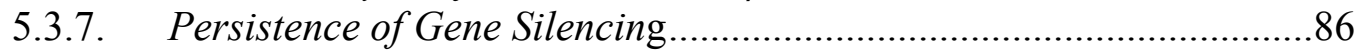

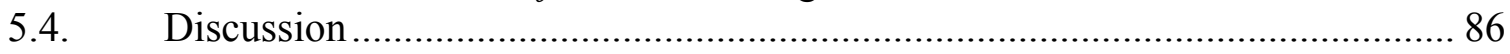

\section{CHAPTER 6. GFAP PROMOTER-DRIVEN RNA INTERFERENCE ON} TGF-B1 TO TREAT LIVER FIBROSIS ..............................................91

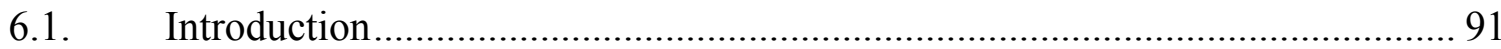

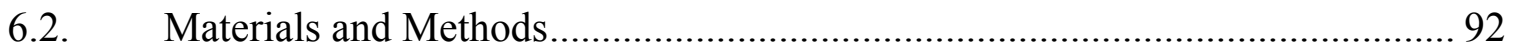

6.2.1. Materials ..........................................................................92

6.2.2. Construction of shRNA Expression Plasmids ………….....................92

6.2.3. Transfection ............................................................................92

6.2.4. Real Time PCR ........................................................................99

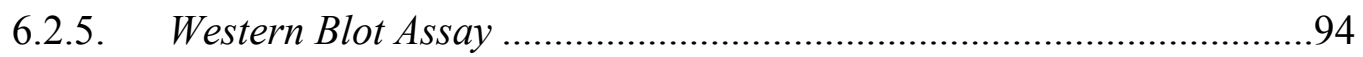

6.2.6. Trypan Blue Staining ………………………................................99

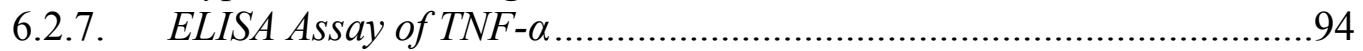

6.2.8. HSC Wound Healing Assay ..............................................................94

6.2.9. Cell Migration Assay ....................................................................95

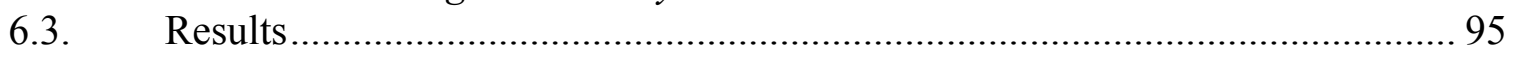

6.3.1. Effect of shRNA Sequence on TGF-B1 Silencing ...............................95

6.3.2. Effect of Promoters and Pri-miRNA Mimics on TGF- $\beta 1$ Gene

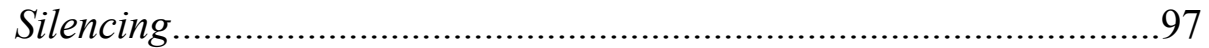

6.3.3. HSC-T6 Proliferation and Apoptosis .................................................97

6.3.4. TGF- $\beta 1$ pGFA-mi-shRNAs Decreases TNF- $\alpha$ Amount in Cell Culture Medium ……...................................................................97

6.3.5. Pmi-shRNAs-TGF- $\beta 1$ Impedes HSC-T6 Migration ............................102

6.3.6. Cell Specificity of GFAP Promoter Driven shRNA Expression..........102 


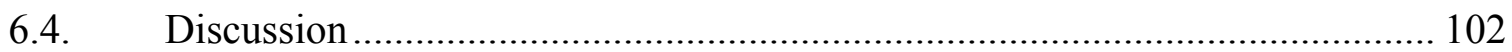

\section{CHAPTER 7. SYSTEMIC ADMINISTRATION OF MICELLAR} FORMULATED PTP, A NOVEL LPA ANTAGONIST, TO

TREAT HEPATIC FIBROSIS.................................................................108

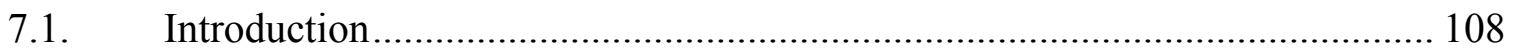

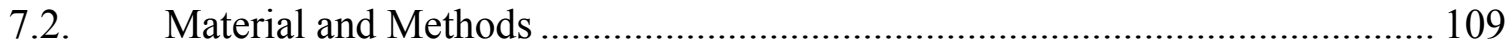

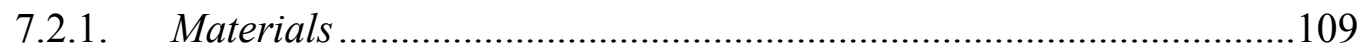

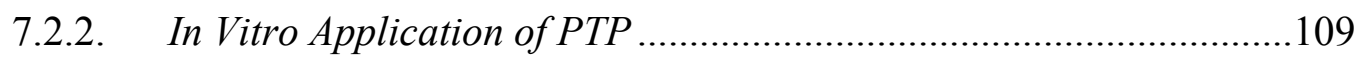

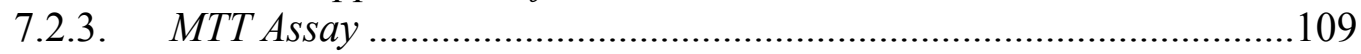

7.2.4. Determination of HSC-T6 Proliferation .............................................110

7.2.5. Measurement of Caspase Activity ..................................................110

7.2.6. Preparation of Micelles ...................................................................110

7.2.7. Determination of Drug Loading Efficiency .......................................110

7.2.8. Particle Size Measurement ..............................................................110

7.2.9. Animal Model and In Vivo Administration ......................................111

7.2.10. Standard Serum Parameters........................................................111

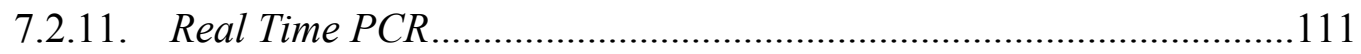

7.2.12. Hydroxyproline Assay .................................................................111

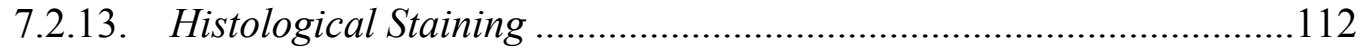

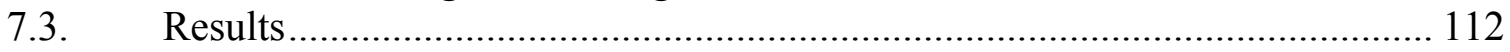

7.3.1. Effects of PTP on HSC-T6 Cell Lines Proliferation and Apoptosis...................................................................................112

7.3.2. In Vitro Characterization of PTP in PEG-PCCL Micells ..................112

7.3.3. Blood Chemistry.........................................................................112

7.3.4. $\quad$ High Dose PTP Micelles Inhibits Both Profibrogens and

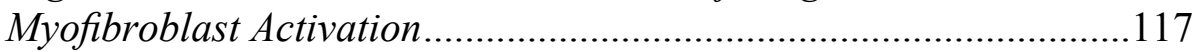

7.3.5. Collagen Levels..........................................................................117

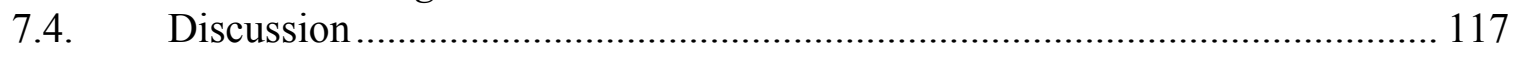

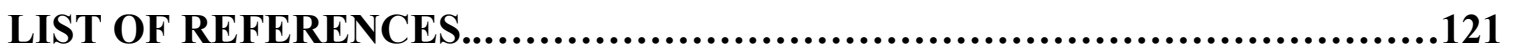

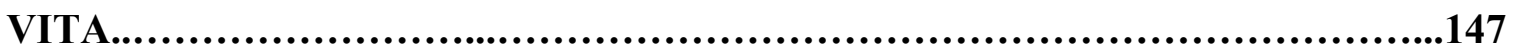




\section{LIST OF TABLES}

Table 2-1 Current clinical trials for oligonucleotides and siRNA .....................29

Table 3-1 Tissue uptake rate index and clearance of ${ }^{32} \mathrm{P}-\mathrm{TFO}$ and M6P-GFLG-HPMA-GFLG- ${ }^{32} \mathrm{P}-\mathrm{TFO}$ after systemic administration into rats ........................................................ 48

Table 5-1 Predesigned siRNAs for rat TGF- $\beta 1$ using BLOCK-iT RNAi designer ................................................ 73

Table 5-2 Sequences of shRNA against different target regions of TGF- $\beta 1 \ldots \ldots \ldots .74$

Table 6-1 Pri-miRNA mimic inserts sequences...............................93

Table 7-1 Particle size measurement of empty and PTP loaded PEG-PCcL micelles ....................................................... 114

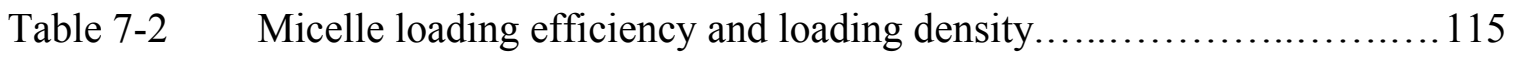




\section{LIST OF FIGURES}

Figure 2-1 Mechanisms of action of antisense-oligodeoxynucleotides (AS-ODNs).... 9

Figure 2-2 Backbone and ribose modifications of ODNs and siRNAs ....................... 14

Figure 2-3 Physiological barriers of oligonucleotides and siRNA............................ 18

Figure 2-4 In vivo efficacy of siRNA formulated with lipidoid $98 \mathrm{~N}_{12}$ with

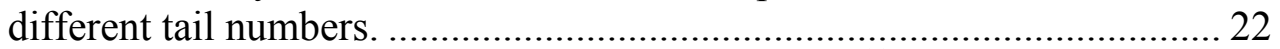

Figure 2-5 Delivery efficiency of M6P-GFLG-HPMA-GFLG- ${ }^{32}$ P-TFO …............... 25

Figure 2-6 Delivery efficiency of Gal-PEG- ${ }^{33}$ P-ODN........................................... 26

Figure 3-1 Synthesis scheme of M6P-GFLG-HPMA-GFLG-TFO. ............................ 34

Figure 3-2 HPLC chromatography of M6P-GFLG-HPMA-GFLG-TFO

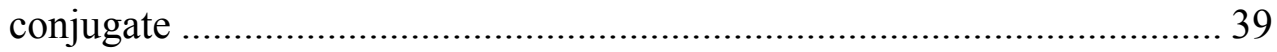

Figure 3-3 Polyacrylamide gel electrophoresis (PAGE) of

M6P-GFLGHPMA-GFLG-TFO ………………………........................ 40

Figure 3-4 TFO dissociation from M6P-GFLG-HPMA-GFLG-TFO by papain......... 41

Figure 3-5 Triplex formation with target duplex DNA after release from M6P-GFLG-HPMA-GFLG ${ }^{32}$ P-TFO conjugate ...................................... 42

Figure 3-6 Inhibition of collagen gene expression .................................................... 44

Figure 3-7 Biodistribution of M6P-GFLG-HPMA-GFLG- ${ }^{32} \mathrm{P}-\mathrm{TFO}$ and free

${ }^{32} \mathrm{P}-\mathrm{TFO}$ after tail vein injection into rats at a dose of $0.2 \mathrm{mg} \mathrm{TFO} / \mathrm{kg}$ of body weight.

Figure 3-8 Concentration time profiles of radioactivity in different organs and the plasma after tail vein injection of M6P-GFLG-HPMA-GFLG- ${ }^{32}$ P-TFO and free ${ }^{32} \mathrm{P}$-TFO into rats at a dose of $0.2 \mathrm{mg}$ TFO $/ \mathrm{kg}$ of body weight.... 46

Figure 3-9 Pharmacokinetic profiles of M6P-GFLG-HPMA-GFLG- ${ }^{32} \mathrm{P}-\mathrm{TFO}$ at 30 min after post vein injection.

Figure 3-10 Effect of fibrosis on hepatic uptake of M6P-GFLG--HPMA-GFLG- ${ }^{32} \mathrm{P}-\mathrm{TFO}$ and HPMA- ${ }^{32} \mathrm{P}-\mathrm{TFO}$ after systemic administration into DMN-induced fibrotic rats 50

Figure 3-11 Effect of excess M6P-GFLG-HPMA on the biodistribution of M6P-GFLG-HPMA-GFLG $-{ }^{32} \mathrm{P}-\mathrm{TFO}$ in fibrotic rats . 51

Figure 3-12 Intrahepatic distribution of M6P-GFLG-HPMA-GFLG- ${ }^{32} \mathrm{P}-\mathrm{TFO}$ in

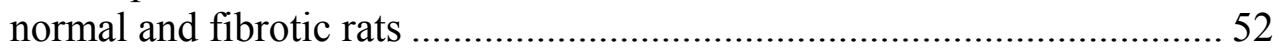

Figure 4-1 NMR characterization of M6P-HPMA-TFO ................................................ 60

Figure 4-2 Measurement of type I collagen and total collagen ................................... 61

Figure 4-3 mRNA and protein expression level screening of fibrotic related

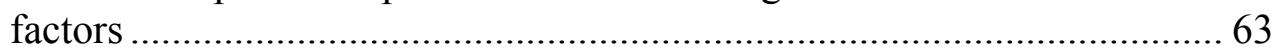

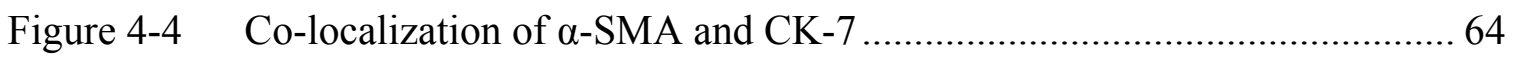

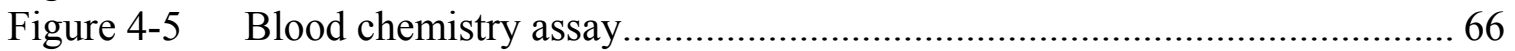

Figure 4-6 CD-11 staining to indicate neutrilphil infiltration...................................... 67

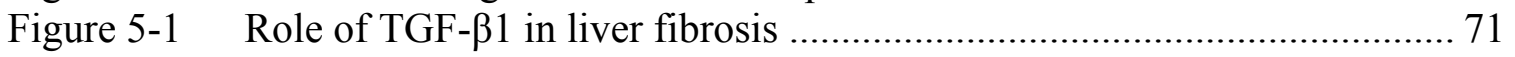

Figure 5-2 The real time PCR plot...................................................................... 76

Figure 5-3 Effect of siRNA sequence on TGF- $\beta 1$ gene silencing ............................... 79

Figure 5-4 Effect of siRNA concentration on TGF- $\beta 1$ silencing ……......................... 80 
Figure 5-5 Western blot analysis for TGF- $\beta 1$ gene silencing after transfection of HSC-T6 cells with Lipofectamine/siRNA complexes

Figure 5-6 Effect of TGF- $\beta 1$ gene silencing on TIMP-1 gene expression after transfection of HSC-T6 cells with Lipofectamine 2000/siRNA complexes.

Figure 5-7 Effect of TGF- $\beta 1$ gene silencing on type $\alpha 1$ (I) collagen and $\alpha$-SMA expression after transfection of HSC-T6 cells Lipofectamine 2000/siRNA-1033, 769 and pool complexes 84

Figure 5-8 Effect of TGF- $\beta 1$ gene silencing on type $\alpha 1$ (I) collagen and TGF- $\beta 1$ expression after transfection of HSC-T6 cells with pshRNA-1033 or pshRNA-769 after complex formation with pyridinium lipid / DOPE

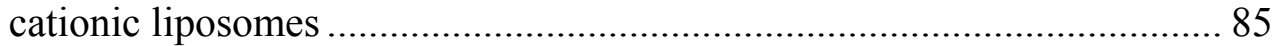

Figure 5-9 Effect of TGF- $\beta 1$ gene silencing on TNF- $\alpha$ and IL- $1 \beta$ secretion .............. 87

Figure 5-10 Persistence of siRNA and shRNA gene silencing..................................... 88

Figure 6-1 Effect of shRNA sequences on TGF- $\beta 1$ gene silencing and its effect on collagen gene expression.................................................................... 96

Figure 6-2 Effects of promoters and pri-miRNA mimics on TGF- $\beta 1$ silencing ......... 98

Figure 6-3 Effects of GFAP-driven TGF- $\beta 1$ pri-miRNA mimics and pri-miRNA cluster mimics on HSC-T6 proliferation ................................................... 99

Figure 6-4 Effects of GFAP-driven TGF- $\beta 1$ pri-miRNA mimics and pri-miRNA cluster mimics on HSC-T6 apoptosis

Figure 6-5 Effects of GFAP-driven TGF- $\beta 1$ pri-miRNA mimics and pri-miRNA cluster mimics on secretion of inflammatory cytokines by HSC-T6 cells.

Figure 6-6 Wound-healing and chamber migration assays........................................ 103

Figure 6-7 Cell specificity of GFAP promoter-driven shRNA expression................. 104

Figure 6-8 The prediction of the secondary structure of the pri-miRNA cluster mimic transcribed from pGFA-mi-shRNA-769+1033 plasmid ................ 106

Figure 7-1 Effects of PTP on HSC-T6 proliferation and apoptosis........................... 113

Figure 7-2 The level of traditional liver disease markers, ALT and AST, were

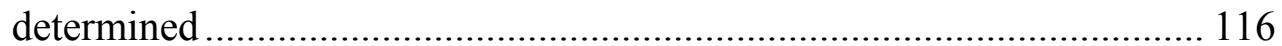

Figure 7-3 mRNA expression level of fibrotic related factors ................................. 118

Figure 7-4 Measurement of type I collagen and total collagen ................................ 119 


\section{LIST OF ABBREVIATIONS}

\begin{tabular}{|c|c|}
\hline$\alpha$-SMA & Alpha-smooth muscle actin \\
\hline $\mathrm{ACN}$ & Acetonitrile \\
\hline apoB & Apolipoprotein B \\
\hline APS & Antiparallel phosphorothioate \\
\hline BBB & Blood brain barrier \\
\hline BSA & Bovine serum albumin \\
\hline CBDL & Common bile duct ligation \\
\hline $\mathrm{CK}$ & Cytokeratin \\
\hline CMV & Cytomegalovirus retinitis \\
\hline DMEM & Dulbecco's modified eagle's medium \\
\hline DOTAP & 1, 2-Dioleoyl-3-trimethylammonium-propane \\
\hline DOTMA & $\mathrm{N}$-[1-(2,3-dioleoyloxy)propel]-N,N,N-trimethylammonium \\
\hline dsRNA & Double-stranded RNA \\
\hline DTT & Dithiothreitol \\
\hline ECM & Extra cellular matrix \\
\hline EMT & Epithelial mesenchymal transition \\
\hline $\mathrm{EtBr}$ & Ethidium bromide \\
\hline FBS & Fetal bovine serum \\
\hline FITC & Fluorescein isothiocyanate \\
\hline Gal & Galactose \\
\hline GPCR & G-protein-coupled receptors \\
\hline HPMA & N-(2- Hydroxypropyl) methacrylamide \\
\hline HSC & Hepatic stellate cell \\
\hline IFN & Interferon \\
\hline IGF & Insulin-like growth factor \\
\hline IL-1 & Interleukin-1 \\
\hline $\mathrm{KC}$ & Kupffer cell \\
\hline M6P & Mannose-6-phosphate \\
\hline miRNA & Micro RNA \\
\hline MMP & Matrix metalloproteinase \\
\hline mRNA & Message RNA \\
\hline MTT & 3-[4,5-Dimethylthiazol-2-yl]-2,5-diphenyltetrazolium bromide \\
\hline ODN & Oligonucleotide \\
\hline PAMAM & Polyamidoamine \\
\hline papM6P & p-Aminophenyl-6-phospho- $\alpha$-D-mannopyranoside \\
\hline PBS & Phosphate buffered saline \\
\hline PDGF & Platelet-derived growth factor \\
\hline pDNA & Plasmid DNA \\
\hline PEG & Poly (ethylene glycol) \\
\hline PEI & Polyethylenimine \\
\hline PLGA & Poly(lactic-co-glycolic acid) \\
\hline PLL & Poly-L-lysine \\
\hline pnpM & p-Nitrophenyl- $\alpha$-D-mannopyranoside \\
\hline
\end{tabular}




$\begin{array}{ll}\text { pnpM6P } & \text { p-Nitrophenyl-6-phospho- } \alpha \text {-D-mannopyranoside } \\ \text { PS } & \text { Phosphorothioate } \\ \text { PSD } & \text { Particle size distribution } \\ \text { RISC } & \text { RNA-induced silencing complex } \\ \text { ROS } & \text { Release oxygen species } \\ \text { SNALP } & \text { Stable nucleic-acid-lipid particle } \\ \text { TFO } & \text { Triplex forming oligonucleotide } \\ \text { TGF } & \text { Transforming growth factor } \\ \text { TIMP } & \text { Tissue inhibitor of metalloproteinase } \\ \text { TNF } & \text { Tumor necrosis factor }\end{array}$




\section{CHAPTER 1. INTRODUCTION}

\subsection{Pathogenesis of Liver Fibrosis and Hepatic Stellate Cells (HSCs)}

\subsubsection{Pathogenesis of Liver Fibrosis}

Excessive deposition of extracellular matrix (ECM) is the common characteristic of liver fibrosis. Liver fibrosis results from sophisticated interplaying among various liver cell types. ${ }^{1}$ Chronic injuries, such as alcoholic abuse, hepatitis, cholestasis and metabolism syndrome, damage the hepatocytes and then trigger infiltration of leukocytes. At the same time, Kupffer cells (KC) are also activated. The filtrated leukocytes and activated KCs continually release oxygen species (ROS) and inflammatory cytokines, which lead to the activation of quiescent HSCs. Part of the activated HSCs go to apoptosis, while part of them survive. The remaining activated HSCs themselves release growth factors, such as transforming growth factor (TGF) and platelet-derived growth factor (PDGF), and further activate quiescent HSCs.

The damage to hepatocytes may be necrosis or apoptosis, ${ }^{2}$ which depends on the type of injury. ${ }^{3,4}$ There are two mechanisms for hepatocytes apoptosis, the extrinsic pathway and the intrinsic pathway. The hepatic diseases involved in the extrinsic pathway of apoptosis include autoimmune or viral hepatitis, alcohol abuse and ischemia/reperfusion associated liver injury. ${ }^{4}$ In contrast, the intrinsic pathway of apoptosis may come from hepatotoxin induced liver injury. ${ }^{5}$ Death of hepatocytes amplifies the inflammation of the liver by recruiting inflammatory cells or directly activating excessive ECM production ${ }^{6}$ by the over made apoptotic bodies. ${ }^{7}$ Meanwhile, the apoptotic bodies by KCs can accelerate hepatocytes apoptosis by inducing expression of death ligands, especially Fas. ${ }^{8}$ In some liver diseases, HSCs can be activated directly without induction of inflammatory by some reagents, such as alcohol metabolites and bile acids. ${ }^{9-11}$ One crucial protein factors triggered by apoptotic bodies is TFG- $\beta 1,{ }^{12,13}$ which is considered as the initial factor in liver fibrosis.

Special soluble growth factors and cytokines, such as IGF-1, ${ }^{14,15} \mathrm{PDGF}^{16}$ and TNF- $\alpha,{ }^{17}$ and fibrotic ECM components ${ }^{18}$ maintain the survival of the activated HSCs. The cytokines like TNF- $\alpha$ may act via the Fas/Fas-ligand (Fas-L) system ${ }^{17}$ to worsen hepatic fibrosis. Compared to the apoptosis inducing ability from other reagents, PDGF has relatively little anti-apoptotic but more proliferating activity. The separation of proliferation and apoptosis in HSCs make the control on HSCs survival regulation easier and safer. Except for the factors directly relating apoptosis and fibrosis, TIMP-1, even not a survival factor, inhibits Matrix metalloproteinases (MMPs) activity ${ }^{19}$ and consequently leads to less HSC apoptosis in an autocrine manner. Part of the increased production of ECM also results from down-regulation of TIMP-1. Besides over-production of ECM, progression of liver fibrosis is caused by remodeling process of ECM, degradation of normal ECM and substitution with scar matrix. ${ }^{20,21}$ The whole process is a kind of complicated network. Activated HSCs, as the principle fibrogenic cells, played the 
critical role in producing major components of ECM, collagen type I and III, in fibrotic livers. ${ }^{22-25}$ This combination of overproduction and remodeling provides a mechanism for the progression of liver fibrosis.

\subsubsection{HSCs}

HSCs, firstly described by Kupper as a vitamin A storing cells, locate in the sub-endothelial space, between the basolateral surface of hepatocytes and the anti-luminal side of sinusoidal endothelial cells.

During liver injury, many properties of HSCs changed eventually. The most significant difference is the lost of lipid droplets. Under microscope, it can be observed that rER enlarges and Golgi apparatus develops more. This phenomenon suggests the up-regulated protein synthesis and indicates activation of quiescent HSCs. ${ }^{26}$ The activated HSCs then play the major role in producing extra amount collagen. It becomes very crucial to focus on HSCs to find out therapeutics. Actually, to treat the hepatic fibrosis, many strategies have been developed based on the understanding of mechanisms, including inhibiting collagen synthesis, inhibiting activation to myofibroblasts, or controlling inflammation. ${ }^{26}$

To control excessive collagen synthesis, we use is one triplex forming oligonucleotides (TFO), which can incorporate with $\mathrm{C} 1$ sequence in the collagen $\alpha$ (I) promoter by hydrogen bonds, leading to transcriptional inhibition on type I collagen. This triplex may prevent both the binding of transcription factors to the gene promoter and duplex unwinding during transcription. TFO was conjugated to M6P-HPMA and showed efficient targeted delivery to HSCs. ${ }^{27}$ Two week short term in vivo i.v. administration suggested M6P-HPMA-TFO might be a potent pro-drug for liver fibrosis.

Transforming Growth factor (TGF- $\beta 1$ ) is believed to be the earliest and most potent stimulus to quiescent HSCs in hepatic fibrosis. ${ }^{28}$ Even though there is no definitive therapeutics basing on anti-TGF- $\beta 1$ found to treat liver fibrosis, many scientific approaches have been achieved to inhibit TGF- $\beta$ action. These approaches include administration of synthetic small molecular compounds, antisense oligonucleotides, small interference RNAs, antioxidants, neutralizing antibodies and etc. In our group, we introduced GFAP promoter driven HSC specific anti-TGF- $\beta 1$ shRNA plasmid to achieve pro-inflammatory control to treat liver fibrosis. ${ }^{29,30}$

The small molecule we applied is one LPA antagonist, PTP, which can interact with LPA receptor on the surface of cells to inhibit the proliferation, which is also one consequence of HSCs activation. 


\subsection{TFO for Liver Fibrosis Treatment}

Triplex forming oligonucleotides (TFOs) perform in a different strategy on gene interference compared with antisense oligonucleotides and siRNAs. Not like antisense, TFO can form triplex with the specific genomic DNA and interfere with transcription, replication, repair and recombination. ${ }^{31}$ TFOs, usually 13-20 nt long, are composed of either polypurine or polypyrimidine, but bind only to the purine-rich strand of their target DNA duplex in the major groove. ${ }^{32}$ According to their base composition, TFOs containing $\mathrm{C}$ and $\mathrm{T}$ nucleotides bind in a parallel and TFOs containing $\mathrm{G}$ and $\mathrm{A}$ or $\mathrm{T}$ nucleotides binds in an anti-parallel orientation to the target strand, respectively. ${ }^{33,34}$

Type I collagen, as the major structural protein in ECM, is an ideal target to treat liver fibrosis. Type I collagen consists of two $\alpha 1$ (I) and one $\alpha 2$ (I) polypeptide chains encoded by the $\alpha 1$ (I) and $\alpha 2$ (I) genes. It was demonstrated that the up-regulated expression of type I collagen by activated HSCs can be at both the transcriptional and post-transcriptional levels.28 It was shown that the synthesis and stability of $\alpha 1$ (I) collagen mRNA were highly increased in the process of hepatic fibrosis. Therefore, there are possibility to prevent fibrosis by inhibiting the transcription of type $\alpha 1$ (I) collagen gene.Mammalian $\alpha 1$ (I) collagen gene promoter contains two contiguous $30-b p$ polypurine tracts $\mathrm{C} 1$ and $\mathrm{C} 2$, located at -141 to -170 and -171 to -200 upstream from the transcription initial site. ${ }^{35}$ It was demonstrated that 18-, 25-, and 30-mer antiparallel phosphorothioate (APS) TFOs specific for $\mathrm{C} 1$ tract inhibit transcription in cultured fibroblasts by forming triplex with the genomic DNA. ${ }^{35,36}$

Actually, systemic delivery of TFOs is been applied a lot for the treatment of both genetic and acquired diseases. In 1998, Fomivirsen (brand name Vitravene), as the first TFO drug, was approved by FDA to treat cytomegalovirus retinitis (CMV) in immunocompromised patients, including those with acquired immune deficiency syndrome (AIDS). The major advantage of TFOs over antisense ODNs and siRNAs is that it interacts with the only gene copy in genomic DNA rather than mRNA, usually existing as hundreds or thousands of copies per cell. Furthermore, specific mRNAs are continuously transcribed from genomic DNA in the nucleus, even though those in the cytoplasm have been silenced. Therefore, inhibition of gene transcription might decrease the mRNA level in a more efficient way at least in some cases.

The TFO investigated in our group have two advantages compared to other TFO. Firstly, this TFO is polypurine TFO without any CpG motifs. Since CpG motifs are considered as immunostimulants in 1997 by Wooldridge et al., ${ }^{37}$ DNA with CpG motifs were utilized to trigger innate immune defense mechanisms. However, stimulation of immune system may worsen liver fibrosis and not good in our case. Secondly, the TFO we are using can forms triplex under physiological conditions, which facilitate triplex formation at target sites. The triplex is more stable to make the silence more efficient. Therefore, the TFO against $\alpha 1$ (I) collagen can be used as a potent anti-fibrotic drug.

We determined the biodistribution of free TFO at whole body, organ, cellular, and subcellular levels after systemic administration in both normal and liver fibrotic rats. ${ }^{38}$ 
The pharmacokinetic profile showed rapid distribution for our TFO to tissues, which was also reported by other groups for PS and G-rich ODNs. ${ }^{39,40}$ PS modification is by far the most extensively studied chemical modification for ODNs to enhance stability and therapeutic time. TFO uptake by tissues was observed as dose dependent but saturated kinetic mode at high dose. The hepatic uptake of the TFO was shown linear at low doses, where nonlinear at higher doses. The hepatic uptake curve of free TFO fit the saturation kinetic equation very well. Liver has the highest affinity to our TFO. The saturation of uptake in liver may suggest a receptor-mediated mechanism for TFOs and lead to redistribution of TFOs to other organs.

However, it was also shown that TFOs are cleared rapidly from the circulation and the accumulation in HSCs is not that promising. To enhance a receptor-mediated TFO delivery to HSCs, BSA was considered as a delivery carrier and M6P as target ligand. ${ }^{41}$ BSA has been utilized for many years as a carrier because it is neither phagocytosed by macrophages in the liver nor excreted by the kidney so as to increase the circulation time of conjugated drugs. The mannose 6-phosphate/insulin like growth factor II (M6P/IGF II) receptor is expressed on the surfaces of HSCs and up-regulated by the stimulation from chronic liver injury. TFO was synthesized to M6P-BSA via a disulfide bond. The percentage of the injected dose accumulated in the liver significantly increased with increase in M6P density. This confirmed the uptake of M6P-BSA- ${ }^{33} \mathrm{P}-\mathrm{TFO}$ is mediated by M6P/IGF II receptor-mediated endocytosis in the liver.

However, because of repeated injections of TFO at high dose to treat liver fibrosis, high molecular weight globular BSA (66430 Da) may not be a suitable carrier for TFO delivery to the HSCs due to possible immune reaction. Furthermore, the high molecular weight of BSA also decreased the total liver up-take, which may results from the narrowed sinusoidal gap in liver after fibrosis. N-(2-Hydroxypropyl) methacrylamide (HPMA) copolymer has shown great potential for delivery of small molecular drugs because of its non-immunogenecity. HPMA has also been used for oligonucleotide delivery by other group, however, no targeting ligands were used achieve site-specific delivery. Therefore, we conjugated M6P to HPMA and then to TFO via GFLG linker, which is known to lysosomal degradable link. ${ }^{27}$ An enzymatic dissociation experiment was applied to M6P-GFLG-HPMA-GFLG-TFO to determine whether TFO could be released from the conjugate. Papain was used as model enzyme because it is a cysteine protease hydrolase enzyme and belongs to the same family as cathepsin B, which is the most important enzyme in the lysosomes to cleave GFLG spacer. It was shown that free TFO release concentration increased with incubation time with papain.

To make sure the TFO released from the conjugate can form a triplex to the target duplex DNA, M6P-GFLG-HPMA-GFLG-TFO was incubated with papain for $24 \mathrm{~h}$ to mimic in vitro release. It was also observed that the TFO released from the conjugate formed a triplex with duplex DNA. After that, in vivo effects of M6P-GFLG-HPMA-GFLG-TFO were determined in common bile duct ligated (CBDL) rats. Following the systemic administration of M6P-HPMA-TFO to CBDL rats three times a week for two weeks, liver samples were analyzed by real time RT-PCR, Western blot, histochemistry and immunofluorescence staining to determine the extent of liver 
fibrosis. M6P-GFLG-HPMA-GFLG-TFO showed the more inhibition ability on collagen synthesis in hepatic fibrotic rats compared to free TFO.

HPMA co-polymers were also proved non-immunogenic. The serum TNF- $\alpha$, an indicator to inflammation, level is slightly different between TFO group and M6P-GFLG-HPMA-GFLG-TFO group after two weeks short term therapeutics.

However, neutrophil infiltration was much less in M6P-GFLG-HPMA-GFLG-TFO group compared to TFO injected group. This result implies that reduced neutrophil accumulation might not only come from exposure to pro-inflammatory factors, which were thought as one reason for neutrophil infiltration. The extra relief of neutrophil infiltration may come from HPMA to protect TFO from the attack by immune system to the intruder.

Another fact unveiled by our data is the continual function of TFO to help the damaged hepatocytes to recover. CK-7 can be expressed in the bile duct epitheliums, no matter in healthy or hepatic fibrotic rats. However, CK-7 can only be expressed in injured hepatocytes before their apoptosis. The staining of CK-7 in liver parenchyma were reduced a lot especially in M6P-GFLG-HPMA-GFLG-TFO group. This result indicates damaged hepatocytes recovered because of the inhibition on synthesizing excessive collagen. M6P-GFLG-HPMA-GFLG-TFO group showed higher effects resulting from target delivery of TFO.

\subsection{RNA Interference on TGF- $\beta 1$ to Treat Hepatic Fibrosis}

After activated by liver injury, HSCs exerts TGF- $\beta 1$ in both autocrine and paracrine ways. ${ }^{42,43}$ For potent fibrogenic effects, autocrine is the most important pattern. Therefore, down-regulation of the TGF- $\beta 1$ produced by HSCs becomes very crucial in treating liver fibrosis.

RNA interference strategies have been frequently applied to TGF- $\beta 1$ silencing recently, since the discovery in 1998. Park and his colleagues constructed a complementary 21-nucleotide sequence, 5'-AACCAAGGAGACGGAATACAG-3', into the plasmid vector pU6-shX, containing promoter regions of the mouse small nuclear RNA U6. ${ }^{44}$ The constructed TGF- $\beta 1$ shRNA expression plasmids were IV injected to fibrotic mice induced by $\mathrm{CCl}_{4}$. After 3 weeks administration of shRNA plasmids after first $\mathrm{CCl}_{4}$ injection, the level of TGF- $\beta 1$, type I collagen, and $\alpha$-SMA in mice were significantly down-regulated, demonstrating that not only the reduced production but also less remodeling of ECM happened. Furthermore, serum ALT/AST level was decreased in the treatment group compared with group without treatment, indicating that TGF- $\beta 1$ siRNA might effectively attenuate liver damage and improve physiological status. It was also reported by our group that several siRNA sequences could silence TGF- $\beta 1$ expression in rat cell line. ${ }^{29}$ Moreover, two selected siRNAs, 769 and 1033, showed pool inhibition of TGF- $\beta 1 \mathrm{mRNA}$. The same results were proved in U6 promoter driven shRNA plasmids. Furthermore, consequent data also demonstrated both TNF- $\alpha$ and IL-1 $\beta$ levels after TGF- $\beta 1$ gene silencing, indicating pro-inflammatory factor relief. 
However, neither U6 nor $\mathrm{H} 1$ promoter can provide spacial or temporal control on silencing TGF- $\beta 1$. The whole body non-specific inhibition of TGF- $\beta 1$ will create disaster. The promoter which can only drive TGF- $\beta 1$ shRNA expression by specific cell types in the body should be utilized for target gene silencing. We utilize glial fibrillary acidic protein (GFAP) promoter to construct an HSC-specific expression plasmid to avoid the side effect of nonspecific TGF- $\beta 1$ gene shut-down. ${ }^{30}$ GFAP promoter driven shRNAs efficiently and specifically inhibit TGF- $\beta 1$ silencing in HSCs.

\subsection{LPA Antagonist for Liver Fibrosis Therapeutic}

LPA is a growth factor-like mediator acting on G-protein-coupled receptors (GPCR) presenting in various physiological fluids. LPA has been reported to involve in many diseases, such as cardiac ischemia, atheriosclerosis, obesity and hepatic fibrosis. It is shown that LPA can activate hepatic myofibroblasts ${ }^{45}$ and increase the proliferation and survival of stellate cells. ${ }^{46,47}$ The correlations between plasma LPA concentration and the histological stages of liver fibrosis markers have been established in patients with chronic hepatitis C. ${ }^{48,49}$ Despite a growing number of LPA antagonists have shown pharmacological ability, few of them have been validated for treatments in animals. It becomes very urgent that to develop more efficient anti-fibrotic drugs that may have important preclinical effects to imply the potent clinical applications in the management of liver fibrosis.

PTP, a novel synthesized LPA antagonist, showed significant inhibition effect on the proliferation of HSC-T6 cell line, which suggests the potent application on liver fibrosis therapeutics. However, the most difficult issue for PTP in vivo administration is the low water solubility. To solve this problem, novel polymers synthesized by our group, ${ }^{50}$ poly(ethylene glycol)-b-poly(carbonate-co-lactide) copolymers, were used to make micellar formulation to enhance solubility. PEG-PCcL micelles were applied to increase the aqueous solubility of PTP. In vivo administration of PTP loaded PEG-PCcL showed therapeutic effects on fibrosis in common bile duct ligated mice. 


\section{CHAPTER 2. DELIVERY AND TARGETING OF OLIGONUCLEOTIDES AND SIRNA}

Oligonucleotides (ODNs) are increasingly being recognized as potential therapeutic agents to modulate aberrant gene expression for treating various diseases, including cancers $^{51-53}$ and viral infections. ${ }^{54,55}$ Concerted efforts have made significant progress in turning these nucleic acids into therapeutics. Apart from immune-stimulation and enzymatic cleavage, the most important feature of ODNs is their ability to block mRNA function by sequence-specific hydridization with target mRNA. ${ }^{56}$ Theoretically, the antisense strategy can be used to target any gene in the body, which makes these nucleic acids achieve broader therapeutic potential than small molecules.

Antisense therapeutic strategies for inhibiting aberrant protein expression have been developed a lot and some of them have already been in clinical trials. ${ }^{57}$ In 2005 , the first antisense ODN drug, Vitravene (Fomivirsen), was approved by the United States Food and Drug Administration (FDA). ${ }^{58}$ After this approval, more and more clinical trials are being conducted, not only for ODNs, but also for other nucleic acids drugs, which are discussed in details in this chapter.

Because of their large molecular weight and negative charge, the delivery of gene drugs is still a big challenge to scientists. For the most popularly used two therapeutic nucleotides, single stranded antisense oligodeoxyribonucleotides (AS-ODNs) and double stranded small interfering RNAs (siRNAs), the molecular weight is at least $6 \mathrm{kDa}$ and $13 \mathrm{kDa}$, respectively. The size of other ODNs is also very big. The large molecular weight prevents them from passing the endothelium smoothly, which is the most important physiological barrier for systemic administration. For many organs and tissues, systemic administration is the only way to be reached by the therapeutic agents in bloodstream. Phosphodiester ODNs are degraded by endo- and exo- nucleases after systemic and local administration. Besides big size and poor biostability, the toxicity induced by these nucleic acids is another big barrier to their therapeutic application. How to achieve efficient gene silencing at non-toxic dose is the most important issue for the success of ODN and siRNA delivery. Various polymer and lipid carriers have been synthesized for their delivery and targeting. In addition, chemical and backbone modifications are used to increase the stability of ODNs.

This chapter discusses about the mode of action, stability and delivery considerations of ODNs and siRNAs as well as ways to overcome their biological barriers. 


\subsection{Single Stranded Oligonucleotides}

\subsubsection{Antisense Oligodeoxyribonucleotides}

An AS-ODN is a short single stranded nucleic acid which binds to specific mRNA and forms a short double stranded hybrid. The length of AS-ODNs is about 13 to 25 base pairs. AS-ODNs can inhibit the translation of mRNA by binding to the mRNA molecules. ${ }^{59}$ The term as "antisense" ODNs is commonly used because their sequences are complementary to target mRNA, which is called the "sense" sequence. The binding affinity and sequence specificity determine the ability of an AS-ODN to form a hybrid with a target mRNA. Binding affinity depends on the number of hydrogen bonds formed between the AS-ODN and the target mRNA sequence. The affinity can be determined by measuring the melting temperature $\left(\mathrm{T}_{\mathrm{m}}\right)$, at which half of the double-stranded hybrid is dissociated into single strands. Binding affinity is also determined by the concentration of AS-ODNs and the ionic strength of the solvent in which hybridization occurs. ${ }^{60}$

Most antisense ODNs follow the two major mechanisms of action (Figure 2-1). One is RNase-H dependent degradation of $\mathrm{mRNA}^{61,62}$ and steric-hindrance of the translational machinery. ${ }^{63}$ RNase-H dependent cleavage of mRNA is the most effective and frequently used mechanism for antisense knockdown. $\mathrm{RNase}-\mathrm{H}$ is a ribonuclease that can recognize RNA-DNA hybrid duplex and cleave 3'-O-P-bond of the mRNA strand in the mRNA-ODN complex. This endonuclease catalyzes the cleavage of RNA via a hydrolytic mechanism with the assistance from an enzyme-bound divalent metal ion. Once the mRNA is cleaved, the AS-ODN dissociates from the duplex and induces another round of RNase-H dependent degradation. Thus, it can be looked as a catalytic process because this prodedure decreases the required concentration of AS-ODNs. Nevertheless, RNase-H recognition is limited to only a few compounds. Some chemically modified AS-ODNs, which have higher stability than unmodified ODNs, cannot be recognized by RNase-H. The steric hindrance mechanism is also termed as 'translational arrest', in which an AS-ODN binds to the single stranded mRNA by Watson-Crick base pairing. This hybrid formation can stop sterically the translation of target mRNA. During the process, the binding of the translational related factors to mRNA may be blocked sterically.

\subsubsection{Triplex Forming Oligonucleotides}

Triplex forming oligonucleotides (TFOs) show different strategy of gene silencing compared with AS-ODNs. TFOs, around 10-30 nt in length, can form triplex with the specific genomic DNA sequences to interfer with transcription, replication, repair and recombination. ${ }^{64}$ TFOs bind to the major groove of duplex DNA, which have runs of purines on one strand and pyrimidines on the other. TFOs are composed of either polypurine or polypyrimidine, but bind only to the purine-rich strand of their target DNA duplex. ${ }^{65}$ According to their base composition, TFOs containing $\mathrm{C}$ and $\mathrm{T}$ nucleotides bind in a parallel and TFOs containing $\mathrm{G}$ and $\mathrm{A}$ or $\mathrm{T}$ nucleotides binds in an anti-parallel 


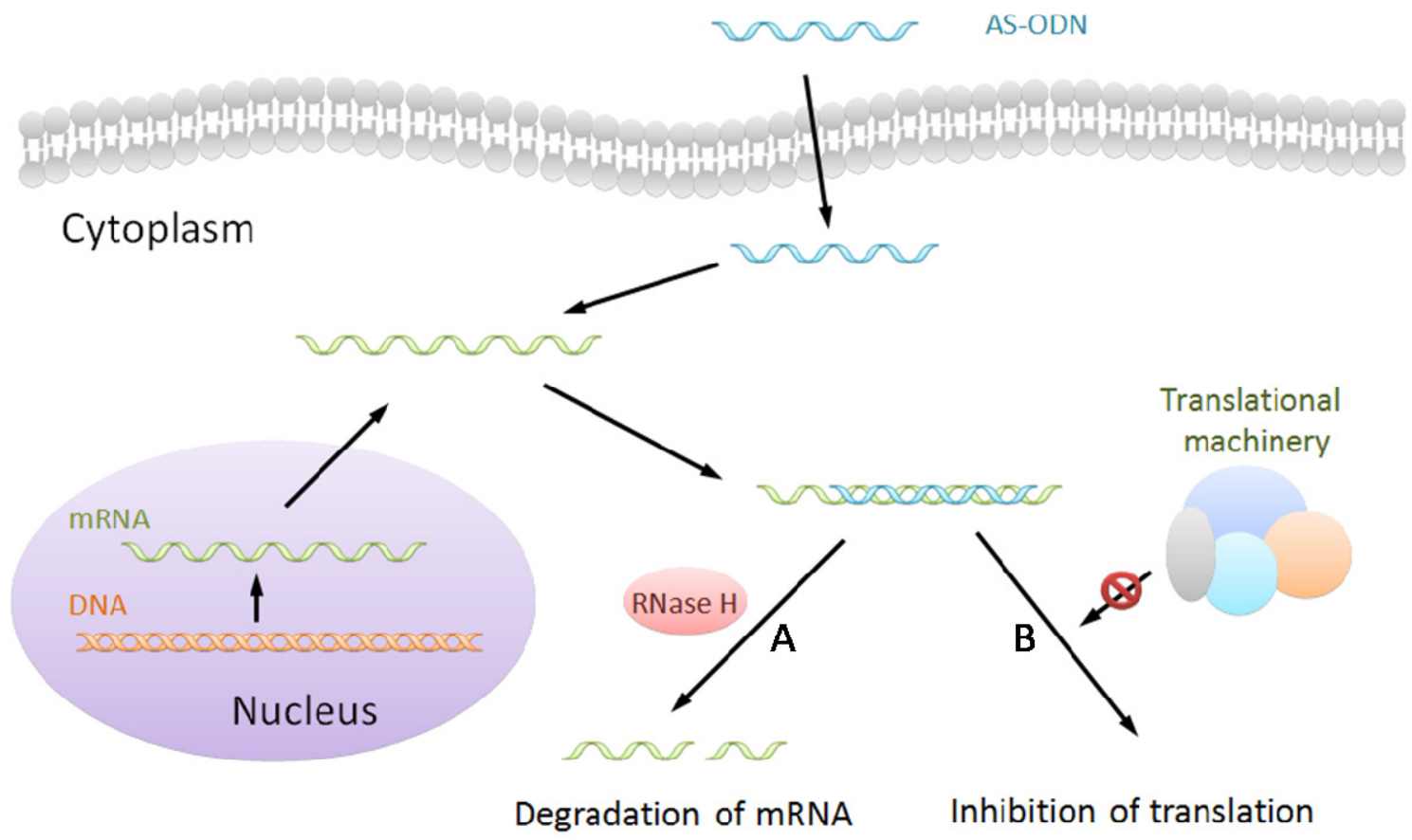

Figure 2-1 Mechanisms of action of antisense-oligodeoxynucleotides (AS-ODNs). A) $\mathrm{RNase}-\mathrm{H}$ dependent degradation of mRNA. In this strategy, RNase-H recognizes RNA-DNA hybrid duplex and cleaves 3'-O-P-bond of the mRNA strand in the mRNA-ODN complex. B) Steric-hindrance of the translational machinery. In this strategy, an AS-ODN binds to the single stranded mRNA by Watson-Crick base pairing strength and forbids the translational machine to move forward. 
orientation to the target strand, respectively. ${ }^{66,67}$ After binding, the transcription of the target gene is blocked.

Transcriptional inhibition gives TFOs several advantages over other gene silencing technologies. ${ }^{68,69}$ Since there are only two copies (two alleles) of a target gene in the genomic DNA, blockage of them means there will be no transcription of DNA into RNA. Since there may be thousands of copies of an mRNA for a specific gene, antisense may not block all these mRNAs. Furthermore, specific mRNAs are continuously transcribed from genomic DNA in the nucleus, even though those in the cytoplasm have been silenced. Therefore, inhibition of gene transcription might decrease the mRNA level in a more efficient way at least in some cases.

\subsubsection{Immunomodulatory Oligonucleotides}

Bacterial DNA can stimulate the proliferation of B cells and the production of inflammatory cytokines by monocytes and other cells, while vertebrate DNA cannot. ${ }^{70}$ Several studies have found that the unmethylated $\mathrm{CpG}$ dinucleotide sequence in DNA is required for this immune-stimulatory activity. ${ }^{71,72}$ Furthermore, single-stranded ODNs containing unmethylated $\mathrm{CpG}$ motifs, which derives from bacterial DNA, are also immunostimulatory, especially with a nuclease-resistant phosphorothioate backbone. CpG ODN, 18-24 bp in length, binds endosomal Toll-like receptor 9 (TLR9) and is taken up by the cells via endocytosis. Once TLR9 is triggered, it may activate numerous signaling transduction pathways and lead to the release of many cytokines, such as IFN- $\gamma$, IL-12, and IL-18. The released cytokines directly stimulate B-lymphocytes, dendritic and natural killer (NK) cells, resulting in innate immunity and antibody-dependent cell cytotoxicity. The signaling pathways activated by CpG DNA in B cells drive them to secrete IL-6, IL-10, and immunoglobulin ${ }^{73,74}$ and to proliferate in a polyclonal T-cell independent manner. ${ }^{75}$ A CpG ODN can also indirectly modulate T-cell responses by increased levels of costimulatory molecules from dendritic cells after application of CpG-ODN. ${ }^{76}$

The activation of Th1-dominant immune responses by $\mathrm{CpG}$ ODN result in the production of several cytokines and $\mathrm{CpG}$ ODNs are promising candicates for treating cancer and allergic diseases as vaccine adjuvants and as immune-therapeutics. A therapeutic application for $\mathrm{CpG}$ ODN is an adjuvant for cancer treatment. Current oncogoing clinical trials combine CpG ODN with chemotherapy or vaccines to treat tumor because $\mathrm{CpG}$ ODN can induce protective immune responses against a lethal tumor challenge. ${ }^{77,78}$ Another important utility of CpG ODN is for treating allergic diseases such as asthma. Due to the favorable Th1-based response induced by CpG ODN, it will redirect the undesirable Th2 responses of allergic disease. ${ }^{72,79}$ Ongoing clinical trials will give us a complete evaluation of this immunomodulatory ODN. 


\subsubsection{Ribozyme and DNAzymes}

A ribozyme, also called RNA enzyme or catalytic RNA, is an RNA molecule that specifically cleaves RNA sequences of choice. Natural ribozymes can form and dissolve covalent bonds by transexterfication, hydrolysis, and peptidyl transfer. ${ }^{80}$ They catalyze not only either the hydrolysis of one of their own phosphodiester bonds or that of bonds in other RNAs, but also the aminotransferase activity of the ribosome. Natural ribozymes can be put into three distinct catogeries: the self-splicing introns (group I and II), ribonuclease P (RNase P), and the small catalytic ribozymes. ${ }^{80-82}$ Groups I and II introns and RNase P belong to the larger, more complicated ribozymes with hundreds of nucleotides in length. The small ribozymes include hammerhead and hairpin ribozymes, which contain 50-70 nucleotides and are commonly used in molecular biology research. ${ }^{83}$

For each category, the specificity of ribozymes for a particular cleavage site is determined by different mechanisms. For the hammerheads, hairpins or group I introns, it is determined by base-pairing between the ribozyme and its RNA target. For the RNase $\mathrm{P}$ category, it is determined by the pairing of a guide RNA with the RNA target. For group II introns, it is determined by the pairing of the ribozyme to its DNA target. For all categories, the target length is another important key.

The ability to engineer small ribozymes that can cleave heterologous RNAs in a sequence-specific manner has enabled the extensive application of hammerhead and hairpin ribozymes as gene knockdown tools and potential therapeutic agents to treat AIDS and cancer patients. ${ }^{84}$ Actually, ribozymes have been investigated to inactivate specific genes for the last two decades and have been used as functional genomic study tools, especially in pre-RNAi era. ${ }^{83}$ Phase I clinical trials using ribozyme to treat AIDS patients have been conducted and got initial success. However, some aspects require further investigation, such as ribozyme stability, ribozyme-substrate colocalization and tissue-specific delivery. ${ }^{85}$

DNAzymes (or deoxyribozymes) are RNA-cleaving analogues of ribozymes. DNAzymes are composed of a catalytic domain flanked by a target-recognition complementary domain. DNAzymes are more stable than ribozymes due to their DNA backbones.

\subsubsection{Nucleic Acid Aptamer}

Aptamers are ODNs or peptides that can bind to their specific targets, ranging from small molecules, ${ }^{86}$ peptides, ${ }^{87}$ amino acids ${ }^{88}$ to proteins. ${ }^{89}$ A nucleic acid aptamer is a linear sequence of nucleotides, typically 15-40 nucleotides long. Mostly, when we talk about aptamers, we refer to nucleic acid aptamers. The intramolecular interaction folds the chain of nucleotides to a complex three-dimensional shape. The shape of the aptamer allows it to bind tightly against the surface of its target. Since some aptamers have tight interaction with their targets, they are also chosen as target ligands for site-specific drug delivery. These aptamers are selected according to pre-defined equilibrium (Kd), rate 
constants (koff, kon) and thermodynamic parameters $(\Delta \mathrm{H}, \Delta \mathrm{S})$ of aptamer-target interaction. Kinetic capillary electrophoresis technology is used for the selection of these smart aptamers. Nucleic acid aptamers are usually created by isolating from synthetic combinatorial nucleic acid libraries by in vitro selection, Systematic Evolution of Ligands by EXponential enrichment (SELEX). The first aptamer-based drug, called Macugen and discovered by OSI Pharmaceuticals, has been approved by the FDA for treating age-related macular degeneration (AMD).

The discovery of the RNA switches made the nucleic acid aptamers be investigated more and more. ${ }^{90-92}$ RNA switches (commonly known as riboswitches) are also capable of binding to small molecule ligands and can control gene expression. Riboswitches are found in the untranslated regions ( $5^{\prime}$-UTR) of mRNA and therefore belong to the noncoding part of the mRNA. Many riboswitches consist of an aptamer domain or a sensor region, which is responsible for ligand binding. Riboswitches modulate gene expression at the level of transcription or translation. Since the similar properties of riboswitches to aptamers, many natural aptamers were found to exist in riboswitches.

\subsection{Double Stranded Therapeutic Oligonucleotides}

\subsubsection{Decoy Oligodeoxynucleotides}

Decoy ODNs are double-stranded DNA sequences which interact with proteins based on Watson-Crick base paring and prevent the targeting transcription factors from their natural interaction partners. Therefore, transcription factors will be removed from their endogenous cis-elements. Decoy ODNs against positive transcription factors can inhibit expression of activated genes and those against negative transcription factors can enhance expression of suppressed genes. ${ }^{93}$ After the first artificial 14 mer E2F decoy ODN, targeted to E2F transcription factor (E2F TF), was synthesized by Morishita et al in $1995,{ }^{94}$ other decoy ODNs to target different proteins such as creb, NF- $\kappa \mathrm{B}$, STAT- 1 , AP-1, have also been found. ${ }^{95-97}$ Decoy ODNs have been applied to treat cancer, renal diseases, viral diseases or cardiovascular diseases because many of these diseases are due to deregulation of different transcription factors. The decoy ODN strategy may not only offer a powerful target-based gene therapy method but also provide a genetic tool to study cellular regulatory processes including upstream transcription regulation and downstream production. ${ }^{93,98}$ In 1996, the FDA approved the clinical application of decoy ODN against E2F to treat neointimal hyperplasia in vein bypass grafts. ${ }^{76}$

\subsection{2. $\quad$ Small Interfering $R N A(\operatorname{siRNA})$}

siRNAs are a class of double stranded RNA sequences, which are 21 nucleotides long. Since the discovery of siRNAs in 1998, more and more investigations have been focused on this RNA interference (RNAi) technology. ${ }^{99}$ RNAi can be initiated not only by siRNA, but also by long double-stranded RNA (dsRNA), plasmid or virus-based short 
hairpin RNA (shRNA), and microRNA (miRNA). Long dsRNA, shRNA, and pre-miRNA are processed by Dicer into 21-23 nt siRNA duplexes with symmetric 2 or 3 nt 3'-overhangs and 5'-phosphate groups. The processed siRNA is incorporated into a protein complex called the RNA induced silencing complex (RISC). Dicer also plays an important role in the early steps of RISC formation. ${ }^{100}$ Argonaute 2, the catalytic component contained within RISC, unwinds siRNA and cleaves the sense strand, which is also called passenger strand. ${ }^{101}$ The activated RISC selectively degrades sequence specific mRNA with the assistance from the antisense strand of siRNA still remaining. ${ }^{102}$ After RISC cleaves the target mRNA, the antisense strand siRNA is not affected. Thus, the RISC can undergo numerous cycles of mRNA cleavage, which further propagates gene silencing. ${ }^{103}$

\subsection{Barriers to Oligonucleotide Based Therapeutics}

\subsubsection{Instability of ODNs and siRNAs}

Native ODNs and siRNAs are rapidly degraded by serum and cellular proteins, and their stability is greatly affected by physiological $\mathrm{pH}$ environments. Clinical applications of these nucleic acids require increasing their stability while retaining their capacity to inhibit aberrant protein expression. One approach is the chemical modifications of ODNs and siRNAs. Figure 2-2 illustrates different structural modifications. These include at backbones, phosphorothioates and boranophosphates, or riboses, 2'position of ribose modification, to enhance their stability without losing their bioactivity.

Since native phosphodiester ODNs are quite unstable, oligonucleotide backbone is often modified not only to improve the stability of ODNs and siRNAs, but also to help them cross the highly impermeable lipid bilayer of the cell membrane. ${ }^{104}$

Phosphorothioate (PS) or boranophosphate modifications of inter-nucleoside linkage are the two types that improve the stability of ODNs and siRNAs.

Phosphorothioates are a variant of natural oligonucleotides in which one of the non-bridging oxygens is replaced by a sulfur atom. This modification lowers the melting temperature $\left(\mathrm{T}_{\mathrm{m}}\right)$ of the mRNA and hybridization efficiency with target mRNA compared to their phosphodiester counterparts. Fortunately, the modified AS-ODN can still be a substrate for RNase-H to trigger an RNase-H dependent mRNA degradation process. Nevertheless, the main drawback of the phosphorothioate modification is that modified nucleotides may induce undesirable effects by binding to plasma proteins. ${ }^{105,106}$

Thioate linkages do not always enhance siRNA stability, because phosphorothioate may reduce the affinity between the two strands of the siRNA duplexes as compared with unmodified RNA. ${ }^{107}$ One interesting study showed that only phosphorothioate modified siRNAs reduced inhibition ability to enhanced green fluorescent protein (EGFP) mRNA. ${ }^{108}$ In this study, the PS linkages were incorporated into the sense strand of siRNA and led to $62 \%$ unmodified siRNA induced inhibition, whereas PS linkages in either the 


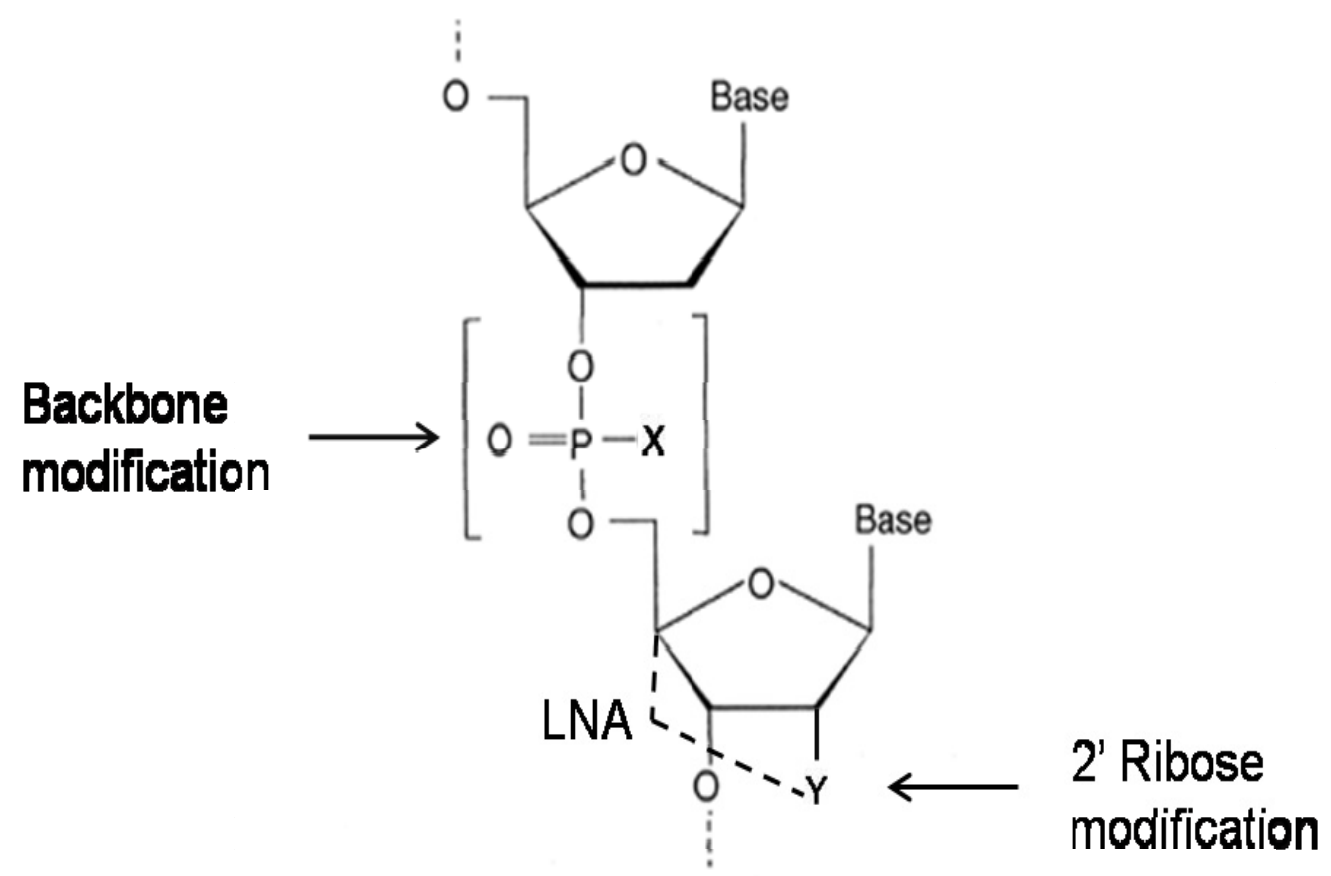

Figure 2-2 Backbone and ribose modifications of ODNs and siRNAs. Backbone and ribose modifications of ODNs and siRNAs. $X$ means backbone modification. $X=-S$ is phosphorothioate, $\mathrm{X}=-\mathrm{BH}_{3}$ is boranophosphate. Y means 2'-position of ribose modification. Including 2'-O-methyl $\left(\mathrm{Y}=-\mathrm{CH}_{3}\right), 2$ '-O-methoxy-ethyl ( $\mathrm{Y}=-\mathrm{O}-\mathrm{CH}_{2}-\mathrm{CH}_{2}-\mathrm{OCH}_{3}$ ), 2'-fluoro ( $\left.\mathrm{Y}=-\mathrm{F}\right)$. In case of Locked nucleic acid (LNA), ribose ring is "locked" by a methylene bridge connecting the 2'-O atom ( $\mathrm{Y}=-\mathrm{O})$ and the 4'-C atom. 
antisense or both strands of the siRNAs led to just less than 50\% inhibition of that observed using unmodified siRNA. However, modification involving both 2'-position and phosphorothiate in the antisense strand showed lower levels of EGFP gene silencing.

Phosphorothioate modification can be easily placed in the nucleic acid sequences at any desired position by two major routes. The first one is the sulfurization in a solution of elemental sulfur in carbon disulfide on a hydrogen phosphonate. ${ }^{109}$ However, the toxicity of carbon disulfide is a barrier for clinical application. The second synthetic method avoids the problem of elemental sulfur's insolubility in most organic solvents and the toxicity of carbon disulfide. This method sulfurize phosphite triesters with either tetraethylthiuram disulfide (TETD) or 3H-1, 2-bensodithiol-3-one 1, 1-dioxide (BDTD) and can yield higher purity phosphorothioates than before. ${ }^{110}$

An alternate backbone modification to increase biological stability of ODNs and siRNAs is the boranophosphate linkage. In boranophosphate ODNs and siRNAs, the non-bridging phosphodiester oxygen is replaced with an isoelectronic borane $\left(-\mathrm{BH}_{3}\right)$ moiety. Boranophosphates have many of the same advantages as phosporothioates. Boranophosphates maintain the ability to make base pair with high specificity and affinity to targets as the unmodified gene drugs. They can also be readily incorporated into DNA and RNA molecules by DNA and RNA polymerases to synthesize stereoregular boranophosphate DNA and RNA. ${ }^{111-113}$ Other additional properties of boranophosphates make them more suitable for clinical use than phosphorothioates. ${ }^{114}$ Since each boranophosphate linkage has a negative charge, the charge distribution of boranophosphates differs from that of normal phosphates and phosphorothioates, and thus increases their hydrophobicity, which facilitates their efficient internalization into the cells. Furthermore, boranophosphate ODNs are minimally toxic to rodents and humans. ${ }^{115}$

Unfortunately, boranophosphate-modified RNAs cannot easily be manufactured using standard chemical synthesis methods. Since boranophosphate bases are incorporated into RNA by in vitro transcription, ${ }^{116}$ which makes specific site selective incorporation of this modification very difficult.

The sugar moiety of oligonucleotides and siRNA can be modified at the 2' position of the ribose, replacing the non-bridging oxygen by 2'-O-methyl (2'-OMe), 2'-O-methoxy-ethyl (2'-MOE) or 2'-fluoro (2'-F).

2'-O-methyl and 2'-O-methoxy-ethyl modifications are the most important members of this class. The DNA/RNA hybrid by AS-ODNs made of these building blocks is very stable. Furthermore, these AS-ODNs are less toxic than phosphorothioates ODNs and even have a slightly enhanced affinity towards their complementary RNAs. ${ }^{117}$ The 2'-O-methyl and 2'-O-methoxy-ethyl modified ODNs are also called the second generation ODNs, while phosphorothioate is called the first generation ODNs. For siRNA, 2'-OMe and 2'-F modified siRNAs have enhanced not only their plasma stability but also their in vivo potency. ${ }^{118}$ 
2'-O-alkyl AS-ODNs cannot trigger RNase $\mathrm{H}$ dependent cleavage of the target mRNA, because the correct width of minor groove of the DNA/RNA hybrid is necessary for the substrate recognition by RNase $\mathrm{H}$. The absence or change of 2'-OH function in DNA/RNA hybrid duplex in the minor groove might alter the interactions between the duplex and the outer sphere $\mathrm{Mg}^{2+}$-water complex in RNase H. ${ }^{119}$ Thus, 2'-O-alkyl AS-ODNs can only take their antisense effect due to a steric block of translation. ${ }^{117,120}$

In contrast to the typical role of AS-ODNs in inhibiting protein expression, blocking of a splice site in an mRNA by an ODN can increase the expression of a specific protein. For example, in one form of $\beta$-thalassemia, a genetic blood disorder, a mutation in intron 2 of the $\beta$-globin gene causes aberrant splicing of $\beta$-globin pre-mRNA and leads to $\beta$-globin deficiency. When 2'-O-methyl ODNs with or without phosphorothioate were targeted to the aberrant splice site, correct splicing was restored generating correct $\beta$-globin mRNA and protein in different mammalian cell lines. ${ }^{121}$

Another interesting study related to 2'-position modified siRNAs has shown that 2'-F modified siRNA may not be more potent than unmodified siRNAs in animals. Even though the modified siRNAs have greatly increased resistance to nuclease degradation in plasma, this increase in stability did not translate into enhanced or prolonged silencing of a target gene in mice after tail vein injection. ${ }^{122}$ In this study, siRNAs modified with 2'-F pyrimidines were functional in cell culture and had greatly increased the stability and prolonged half-life in human plasma, compared to unmodified siRNAs. Although the 2'-F modified siRNAs inhibited the expression of the target gene in mice, the inhibitory ability of modified siRNAs was not better than that of unmodified ones. The reason may be that 2'-F modified siRNAs and unmodified siRNAs have different non-specific binding tendency in vivo.

Locked nucleic acid (LNA), also referred to as inaccessible RNA, is a family of conformationally locked nucleotide analogs in which the ribose ring is "locked" by a methylene bridge connecting the 2'-O of the ribose with the 4'-C atom. LNA nucleotides can be mixed with DNA or RNA bases in the ODNs and siRNAs whenever desired. LNA ODNs showed an enhanced stability against nucleolytic degradation ${ }^{123}$ and high target affinity. However, LNA appears to show hepato-toxicity as indicated by serum transaminases concentration, organ and body weights. ${ }^{124}$ LNA were also compatible with siRNA intracellular machinery, increased nuclease resistance, and furthermore, reduced sequence-related off-target effects. ${ }^{107,125}$

\subsubsection{Non-specific Binding and Toxicity}

Non-specific binding (commonly known as off-target effect) of ODNs has troubled the scientists since the beginning of this area, even though one off-target effect initiated by $\mathrm{CpG}-\mathrm{ODNs}$ is now being investigated for therapeutic purposes. Off-target reaction with un-intended sequences is also adversely affecting the progress of RNAi technology. These off-target effects come from the binding not only to non-target sequences, but also to the plasma proteins. 
High concentration of ODNs and siRNAs increases their interaction with non-target sequences, leading to toxic side effects. Off-target effects of AS-ODN usually occurres if the concentration is higher than $200 \mathrm{nM}{ }^{126}$ Semizarov et al. also found similar results for siRNA that the specificity of siRNA is concentration dependent. ${ }^{127}$ When the concentrations of siRNA reached $100 \mathrm{nM}$, siRNA nonspecifically stimulated a significant number of apoptosis related non-target genes. These evidences suggest that gene silencing experiments should be designed under the concentration threshold. Since a siRNA recognizes its targets by sequence complementarity, potential off-target effects of siRNAs could also be decreased by the proper selection of siRNA sequences within 100 nt from the 5 ' termini of the target mRNA. ${ }^{128}$ Off-target effects of siRNA can also be minimized by using smart pools of siRNAs, which means the mixture of siRNAs targeting different regions of mRNA of the same gene and reduces the off-target effect induced by only one siRNA in the same total concentration, but that is higher than single one in the pool. Moreover, 2'-MOE modification was also reported to reduce the 'off-target' effect, ${ }^{129}$ suggesting that proper chemical modifications can reduce the off-target effects.

The binding to plasma protein also affects the target specificity and gene silencing efficiencies of ODNs and siRNAs. Even though PS modification increases the stability of ODNs and siRNAs, it promotes their binding to plasma proteins. To minimize binding to plasma proteins, while still maintaining high stability, ODNs are often partially phosphorothioated. Since the main reason for the degradation of ODNs and siRNAs is exonuclease attack, the entire sequence can be protected by a few phosphorothioate linkages at the terminals. The incorpration of several central phosphorothioate residues in a potent AS-ODN, that is termed as 'gapmers', can still activate RNase H dependent cleavage but retaining many of the valuable properties of the unmodified nuclei acid sequence. $^{130}$

Besides off-target effects, systemic administration of siRNA duplexes may lead to innate immuo-response, inducing high level of inflammatory cytokines, such as tumor necrosis factor alpha (TNF- $\alpha$ ), interleukin-6 (IL-6) and interferons (IFNs), and innate immunity, which can be mediated by Toll like receptors (TLRs). ${ }^{131}$ Innate immunity of siRNAs can also be triggered by non-TLR-mediated pathways, such as siRNA binding to retinoic acid inducible gene 1 (RIG1) in the cytoplasm. 2'-OMe modified siRNAs have shown to prevent recognition by the innate immune system. ${ }^{132}$ Combined with the ability of reducing off-target effects of siRNA, 2'-OMe modification of ribose does reduce the toxicity of synthetic siRNAs.

\subsubsection{Physiological Barriers}

Different physiological structures prevent the nucleotides from being delivered to the target successfully. The most important three physiological barriers are capillary endothelia, endosome/lysosome membranes and nuclear membranes (Figure 2-3). 


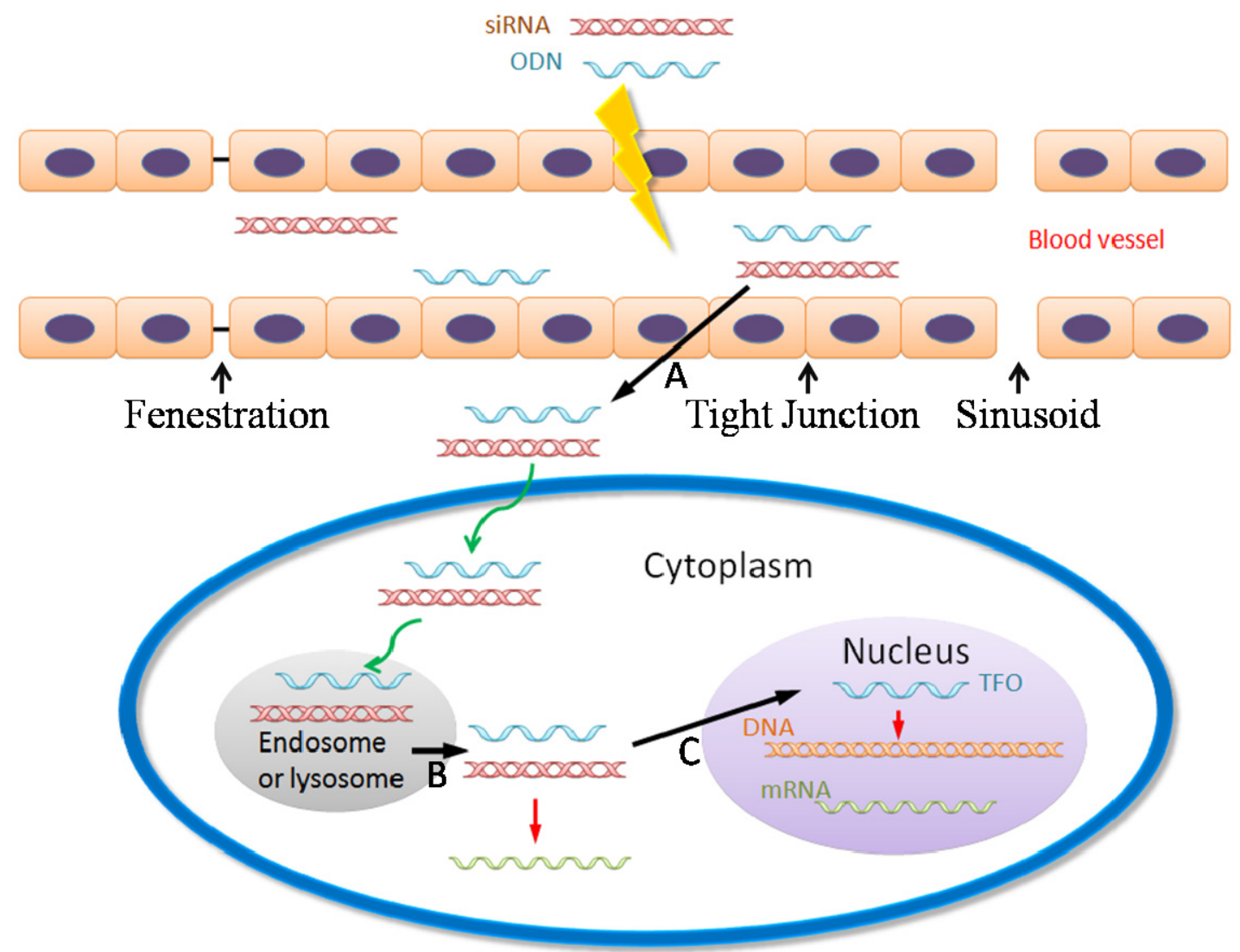

Figure 2-3 Physiological barriers of oligonucleotides and siRNA. A) Capillary endothelium. B) Endosomal and lysosomal membranes. C) Nuclear membranes. 
The most important physiological barrier to ODN and siRNA delivery is the capillary endothelium, which is a thin monolayer of cells that line the interior surface of blood vessels with or without special basement membranes. Although the endothelial cells are also the therapeutic targets in some diseases, for example, high blood pressure, in most situations ODNs and siRNAs have to extravastate the endothelium to reach tissue parenchymal cells.

The capillary endothelia in various organs and tissues have different extravastation properties according to the morphology and continuity of the endothelial layer and the basement membrane. ${ }^{133}$ The capillary endothelia, found in cardiac, smooth muscles, lung, skin, subcutaneous and mucous membranes, have little fenestrations because these endothelial cells are joined by tight junctions and continuous subendothelial basement membranes. ${ }^{134}$ Therefore, the particles larger than $2.0 \mathrm{~nm}$ are very hard to be extravasated. In some organs, the endothelial cells with tight junctions make unique structure for self-protection and filter function, such as the blood-brain barrier (BBB). The capillary endothelia, found in kidney, small intestine and salivary glands, are composed of fenestrated endothelial cells and a continuous basement membrane. ${ }^{135}$ Except the glomerular capillaries in kidney, these type capillaries allow the extravasation of particles less than $11 \mathrm{~nm}$ in diameter. For the glomerular capillaries, the effective permeation is allowed for the particles smaller than $30 \mathrm{~nm}$. Moreover, due to the negative charges on the glomerular capillary walls, the extravasation is also affected by the molecular charge of ODNs and siRNAs or their formulated complexes. The capillary endothelia, found in the liver, spleen and bone marrow, have fenestrations up to 150 $\mathrm{nm} .{ }^{134}$ In addition, the basement membrane is absent in the liver and discontinuous in the spleen and bone marrow. All of these properties allow the ODNs and siRNAs to pass through the sinusoidal gaps of the liver to reach the hepatocytes.

Inflammation, tumor formation and fibrosis lead to changes in endothelial barriers. Inflammation facilitates the distribution of ODNs and siRNAs to the interstitial spaces, not only due to the increased fenestration between endothelial cells, ${ }^{136}$ but also due to the increased permeability of the endothelial cells themselves. ${ }^{137}$ In case of solid tumors, many newly formed tumor vessel endothelial cells are poorly-aligned with wide fenestrations, lacking a smooth muscle layer. Combined with other factors, such as non-effective lymphatic drainage, solid tumor tissues have enhanced permeability and retention (EPR) effect, which will allow efficient distribution of ODNs and siRNAs to the tumor cells. In these tissues, low molecular weight drugs are cleared with short plasma half-lives, with little distribution to the tumor, whereas high molecular weight drugs or nanoparticles accumulate in inflammatory and tumor tissues eventually. ${ }^{138}$ However, for liver fibrosis it is a different case. After fibrosis, sinusoidal gaps, which are up to $150 \mathrm{~nm}$ in width under non-pathological conditions, are almost closed, leading to decreased free exchange flow between hepatocytes and sinusoidal blood. This change makes the delivery of ODNs and siRNAs formulated in large size nanoparticles much more difficult. Cheng et al. showed that the accumulation of TFO in fibrotic rat livers decreased from $44 \%$ to $34 \%$ of total IV injection compared to normal liver. ${ }^{139}$ 
Another barrier is endosomal and lysosomal membranes. Endocytosis appears to be the major pathway for the cellular uptake of ODNs and siRNAs. ${ }^{140}$ After endocytosis, ODNs and siRNAs have to escape from the endosome and lysosome before being degraded. There are several strategies for ODNs and siRNAs to escape into the cytoplasm, including destabilization of endosomal compartment, ${ }^{141}$ an exchange of cationic lipids with anionic phospholipids in cytoplasm-facing membrane monolayer, ${ }^{142}$ and endosomolysis by osmotic swelling. ${ }^{143}$ For cationic liposome formulated ODNs and siRNAs, the choice of proper co-lipids, which can disrupt the endosomal or lysosomal membrane, help them to escape more efficiently.

Unlike AS-ODNs and siRNAs, TFOs must enter the nucleus to form triple helix with genomic DNA to inhibit transcription. Although particles smaller than $30 \mathrm{kD}$ can pass through the nuclear pore complex by passive diffusion, intra-nuclear concentration of TFOs must be high enough to compete with transcriptional factors at the same genome gene site. Fortunately, many sorting signals, such as nuclear localization signal (NLS) peptides, have been discovered, ${ }^{144}$ which can facilitate the nuclear translocation of proteins and RNAs.

\subsection{Synthetic Carriers for Nucleic Acid Delivery}

\subsubsection{Complex Formation}

Cationic lipids are by far the most commonly used transfection agents for ODNs and siRNAs. Cationic liposomes can be used to either encapsulate these nucleic acids or form lipid/nucleic acid lipoplexes. Cationic liposomes have been used for nucleic acid delivery for more than 20 years. In 1987, the efficiency of the cationic lipid N-[1-(2,3-dioleyloxy)propyl]-N,N,N-trimethlyl ammonium chloride (DOTMA) to deliver both DNA and RNA into mouse, rat and human cell lines was firstly investigated. ${ }^{145}$ However, many of the cationic lipids used in early clinical trials, such as 3-[N-(N,N -dimethylaminoethane)-carbamoyl] cholesterol (DC-Chol), 1,2-dimyristyloxypropyl-3-dimethyl-hydroxy ethyl ammonium bromide (DMRIE), and GL-67, did not show high efficiency in vivo. Therefore, more and more cationic lipids were synthesized and tested for nucleic acid delivery.

Recently, for in vivo siRNAs studies, Morrissey and colleagues reported the inhibition of hepatitis B virus (HBV) replication in mice after systemic administration of stable nucleic acid/lipid particles (SNALPs) that targeted HBV mRNA (HBV263M). Dose-dependent reduction in serum HBV DNA levels was observed seven days after three daily intravenous injections of anti-HBV siRNA SNALP at the dose of 3 $\mathrm{mg} / \mathrm{kg} /$ day. Furthermore, the similar reduction in HBV replication had been maintained for more than six weeks. Zimmerman and colleagues also encapsulated ApoB-specific siRNAs in SNALP and injected intravenously to cynomolgus monkeys at doses of 1 or $2.5 \mathrm{mg} / \mathrm{kg} .{ }^{146}$ A single siRNA injection resulted in dose-dependent silencing of ApoB mRNA expression in the liver $48 \mathrm{~h}$ after administration, with gene silencing of more than 
$90 \%$. The silencing effect persisted for 11 days at the highest administered dose of 2.5 $\mathrm{mg} / \mathrm{kg}$.

In our laboratory, Zhu et al. synthesized a series of pyridinium lipids with a heterocyclic positively charged ring linked to different types of fatty acids via ester or amide spacers. ${ }^{147}$ These lipids showed enhanced in vitro transfection efficiency both for plasmid and siRNAs. Transfection efficiency of these pyridionium lipids was dependent on their hydrophobic chain lengths used. Length beyond $16 \mathrm{C}$ decreased the transfection efficiency. Increase in the aliphatic chain length of amphipathic compounds is known to increase both the phase transition temperature and bilayer stiffness of the resulting vesicles, and having a stiff bi-layer is unsuitable for membrane fusion. ${ }^{148}$

Transfection efficiency of cationic liposomes can also been improved by conjugation to targeting ligands. When vitamin-A-coupled liposomes were used for delivery of anti-gp46 siRNA dimethylnitrosamine (DMN) induced liver fibrotic rats, ${ }^{149}$ there was prolonged survival of liver fibrotic rats in a dose dependent manner. Rats were almost cured of liver fibrosis after administrations.

Lipidoids is another class of lipid-like material to deliver siRNAs to the liver after systemic administration. ${ }^{150}$ The basic synthesis idea is to conjugate alkyl-acrylates or alkyl-acrylamides to primary or secondary amines. Among the huge library of lipidoids, $98 \mathrm{~N}_{12}-5$ (5-tail) was found to be optimal for in vivo delivery of siRNA compared to other similar compound (Figure 2-4). Almost $80 \%$ of the injected dose distributed to the liver and could induce persistent gene silencing without loss of activity following repeated administration. The lipidoids showed high safety and efficiency in all the three animal models, mice, rats and nonhuman primates.

Various cationic polymers including polyethyleneimine (PEI) ${ }^{151}$ poly(L-lysine) (PLL), ${ }^{152}$ poly(amidoamine) (PAMAM) dendrimer, ${ }^{153}$ polyallylamine 104 and methacrylate/methacrylamide polymers ${ }^{154}$ have been synthesized for nucleic acid delivery and targeting. Polymeric carriers hold promise due to their versatile chemistries, targetability and low toxicity, but they usually have poor transfection efficiency.

Among various cationic polymers, PEI remains very popular, which has either branched or linear form. PEI is available in a broad molecular weight ranging from less than $1 \mathrm{kDa}$ to $1.6 \times 10^{3} \mathrm{kDa}$, but PEI of 5 to $25 \mathrm{kDa}$ are widely used for gene transfer since high molecular weight PEI is cytotoxic to the cells. ${ }^{155,156}$ Low molecular weight PEI, by contrast, has shown low toxicity in cell culture studies. ${ }^{157,158}$ Forrest et al. has combined the low toxicity properties of low molecular weight PEI with the high transfection efficiency of high molecular weight PEI by coupling low molecular weight PEIs $(800$ Da) together to form conjugates of 14-30 kDa using short diacrylate cross-linkers. ${ }^{159}$ These degradable polymers have similar DNA-binding properties to commercially available $25 \mathrm{kDa}$ PEI, but exhibit two- to sixteen-fold higher transfection efficiency and are essentially nontoxic. Other strategies to reduce the toxicity and improve the stability are synthesizing PEI with graft copolymers such as linear poly(ethylene glycol) (PEG) ${ }^{160,161}$ or glycosylated. ${ }^{162}$ Petersen et al. have synthesized two series of 

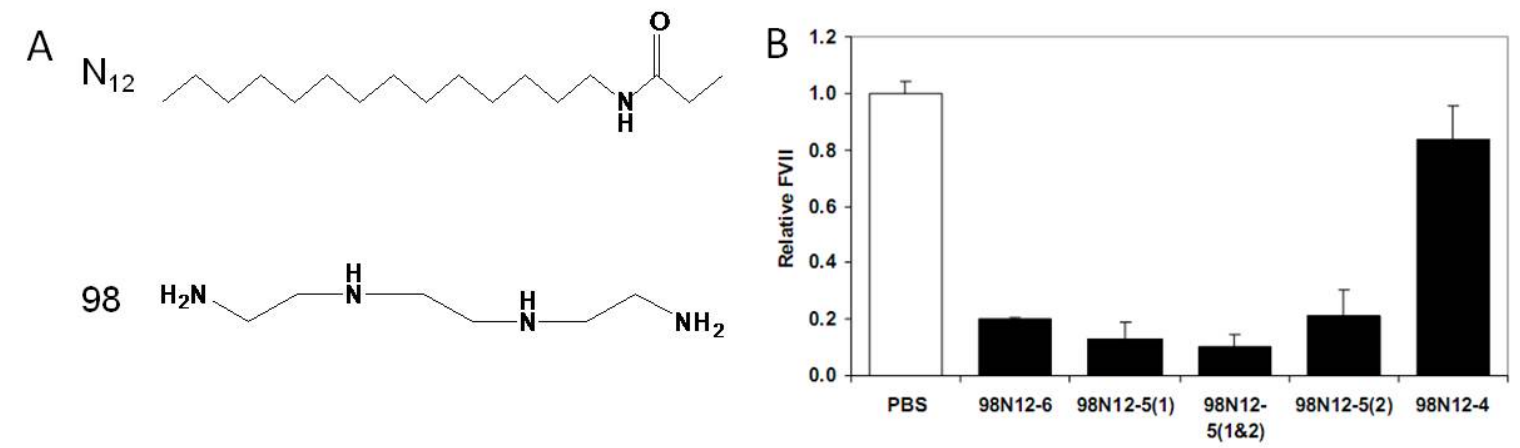

Figure 2-4 In vivo efficacy of siRNA formulated with lipidoid $98 \mathrm{~N}_{12}$ with different tail numbers. A) Structure of $\mathrm{N}_{12}$ and 98 lipidoids; B) Transfection efficiency of different $98 \mathrm{~N}_{12}$ compounds. From left to right are blank control, the 6-tail compound $\left(98 \mathrm{~N}_{12}-6\right)$, one isomer of the 5-tail compound, mixture of the two 5-tail isomers, another isomer of the 5-tail compound and the 4-tail compound. Factor VII-targeting siRNA was formulated using these compounds and administered to C57BL6 mice at $2.5 \mathrm{mg} / \mathrm{kg}$ via single i.v. bolus injection. Twenty-four hours after administration, serum factor VII protein levels were quantified. 
polyethylenimine-graft-poly(ethylene glycol) (PEI-g-PEG) block copolymers112 by grafting PEI $(25 \mathrm{kDa})$ to PEG $(5 \mathrm{kDa})$ or a series of PEG of $550 \mathrm{Da}$ to $20 \mathrm{kDa}$. The size and morphology of resulting polyplexes were drastically changed. PEG $(5 \mathrm{kDa})$ significantly reduced the diameter of complexes from $142 \pm 59$ to $61 \pm 28 \mathrm{~nm}$. Copolymers with PEG (20 kDa) yielded small, compact complexes with DNA while copolymers with PEG (550 Da) resulted in large and diffuse structures. The zeta-potential of complexes was reduced with increasing degree of PEG grafting if molecular weight was more than 5 $\mathrm{kDa}$. Cytotoxicity was independent of PEG molecular weight but was affected by the degree of PEG substitution. The copolymers with more than six PEG blocks formed DNA complexes of low toxicity.

Dendrimers consist of a central core molecule as roots, from which some tree-like branches originate in an ordered way. This unique architecture gives dendrimers various distinctive properties. The intrinsic viscosity of dendrimer solution does not increase linearly with mass, ${ }^{163}$ which make the application of the formulation by polymer dendrimers much easier. Furthermore, the tree-like structure can maximize the exposed surface area, which facilitates the interaction between dendrimers and nucleic acids. The multiple surface groups of dendrimer allow conjugation of various targeting ligands and other moieties to confer site-specificity and reduced toxicity.

Among various dendritic polymers, polyamidoamine (PAMAM) dendrimers have recently attracted interest for nucleic acid delivery because of their well-defined surface functionality, low polydispersity, good water solubility, and non-toxicity. Bielinska et al. transfected ODN/PAMAM complexes into D5 mouse melanoma and Rat2 embryonal fibroblast cell lines in vitro. ${ }^{164}$ The ODN/dendrimer complexes showed good silencing effect with very little cytotoxicity compared to Lipofectamine and DEAE dextran complexes. PAMAM dendrimers also showed strong binding affinity for siRNA molecules. ${ }^{165}$ These nondegraded dendrimers condensed siRNAs into nanoscale particles and protect them from enzymatic degradation, leading to gene silencing.

\subsubsection{Bioconjugation}

Most cationic lipids and polymers used as transfection agents are toxic, which limits their clinical applications. To avoid the use of polycations, Rajur et al. conjugated ODNs to asialoglycoprotein (ASGP) using sulfosuccinimidyl 6-[3'-(pyridyldithio) propionamido] hexanoate (sulfo-LC-SPDP). ${ }^{166}$ Direct conjugation of molecules to the ODNs often tends to disturb the bio-ability of the ODNs, which is essential for errant protein knocking down. Therefore, ODNs were covalently conjugated to carbohydrate cluster for specific delivery to the hepatocytes ${ }^{167}$ and other cells.

Various carriers are also utilized for conjugation of siRNAs, including cholesterol and $\mathrm{VE}$, and PEG. The site for conjugation is crucial for siRNAs. The integrity of the 5 '-terminus of the antisense strand of siRNA which is complementary to the target mRNA and incorporated into RISC to initiate the mRNA cleavage, is crucial for initiation of RNAi. ${ }^{168}$ Therefore, the 5'-terminus cannot be used for conjugation. Either the 3 '- or 
5 -terminus of the sense strand is generally used for conjugation. Moreover, the linkages between carriers and siRNAs should be acid or enzyme sensitive to allow complex formation between RISC and siRNA in the cytoplasm. Since matrix metalloproteinase 1 (MMP1) is upregulated in liver fibrosis, a special six amino acid peptide, substrate for MMP1, is used as an enzyme-sensitive linker.

Then, how to decrease the toxicity and increase the target efficiency of therapeutic oligonucleotides? The most important strategy is the addition of targeting ligands. Many diseases change the physiology of cells, such as special-receptor upregulation. For example, liver fibrosis leads to the activation of hepatic stellate cells (HSCs) which affects the liver architecture and eventually liver function. Since Mannose-6-phosphate (M6P) receptors of HSCs get upregulated upon HSC activation, Mahato's lab synthesized M6P-bovine serum albumin (M6P-BSA) and conjugated the TFOs via a disulfide bond for enhanced TFO delivery to the HSCs. ${ }^{169}$ They also checked the influence of the M6P number per conjugate molecule on the biodistribution and hepatic uptake of M6P-BSA-TFO. ${ }^{170}$ The molar ratio of M6P: BSA to 21 and 27 resulted in an increased liver accumulation to $52.6 \%$ and $67.4 \%$, respectively, whereas free TFO showed liver accumulation about $45 \%$.

Since the treatment of liver fibrosis may require repeated injections of TFO at high doses, high molecular weight globular BSA $(\mathrm{MW}=67000)$ may not be a suitable carrier for TFO delivery to the HSCs due to possible immune reaction. N-(2-Hydroxypropyl) methacrylamide (HPMA) copolymer has shown great potential for delivery of small molecular drugs. ${ }^{171}$ Therefore, Yang et al. synthesized M6P-GFLG-HPMA-GFLG-ONP and conjugated it to TFO via GFLG linker, which is a lysosomally degradable tetrapeptide linker and known to be cleaved by lysosomal enzymes, allowing TFO release the cytoplasm after cellular uptake. The HPMA copolymer (MW=40000Da) conjugate of TFOs increased the liver accumulation of the TFO to $80 \%$ of the total injected dose, which is quite high compared to free TFO (45\%) (Figure 2-5). ${ }^{172}$

PEGylation is known to significantly enhance the ODN stability against exonuclease and reduces renal clearance compared to unmodified ODNs. ${ }^{173}$ Conjugation of PEG to ODNs can decrease the RES clearance of administered nucleotides and prolong the circulating time of them in blood. ${ }^{174,175}$ Zhu et al. conjugated Gal-PEG to ODNs via an acid-labile linker. The conjugation of PEG prolonged the circulation time, but also decreased the binding of ODNs, which were G-rich PS ODNs, to the serum protein. With the assistance of galactose as the ligands, Gal-PEG-ODNs were delivered to the hepatocytes (Figure 2-6). After endocytosis, the low $\mathrm{pH}$ in the endosome made the $\beta$-thiopropionate linkage cleaved and ODN were released from conjugate gradually. After conjugation with PEG, the elimination half life of ODNs increased from 34 min to 118 $\min .139,175$

In 2004, cholesterol was covalently linked to the 3 '-terminus of the sense strand of siRNAs which contain selective stabilizing modification and were designed to target the apolipoprotein B mRNA. ${ }^{176}$ In this case, Soutschek and his colleagues used a pyrrolidone linkage that is not bio-cleavable. Cholesterol-siRNA conjugate (Chol-siRNA) showed not 
A

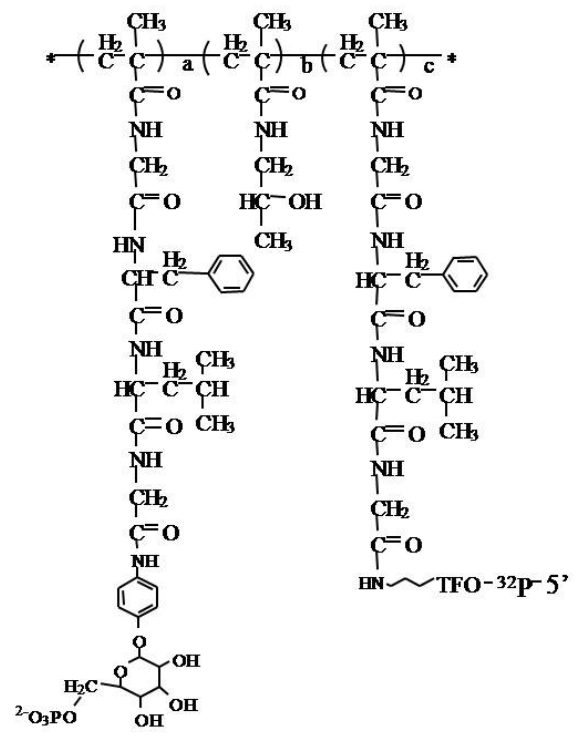

B

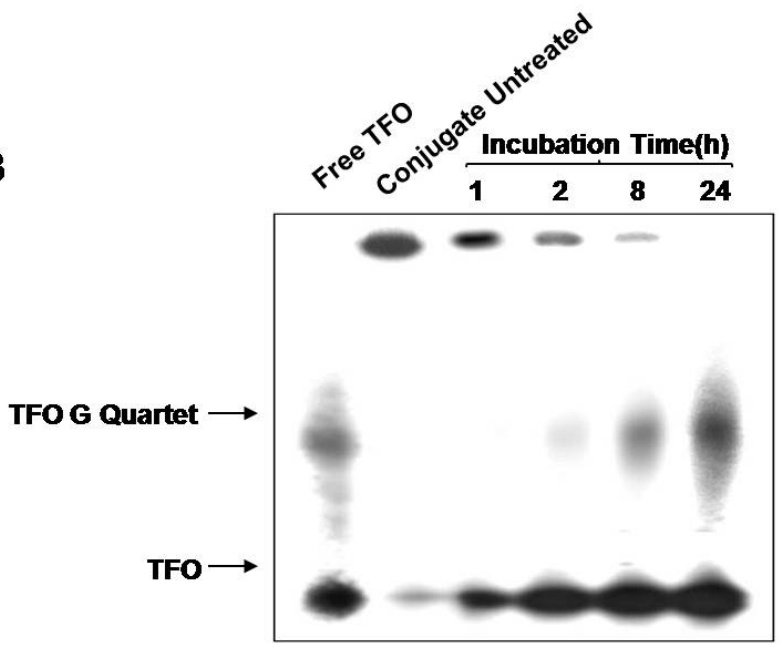

C

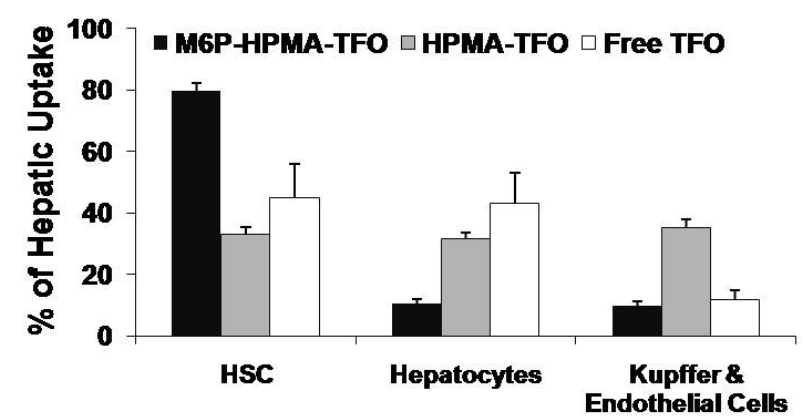

Figure 2-5 Delivery efficiency of M6P-GFLG-HPMA-GFLG- ${ }^{32}$ P-TFO. A) Structure of M6P-GFLG-HPMA-GFLG- ${ }^{32}$ P-TFO. B) Enzymatic dissociation of ${ }^{32} \mathrm{P}-\mathrm{TFO}$ from M6P-GFLG-HPMA-GFLG- ${ }^{32}$ P-TFO by papain. C) Intrahepatic distribution of M6P-GFLG-HPMA-GFLG- ${ }^{32} \mathrm{P}-\mathrm{TFO}$ in fibrotic rats. Cells were isolated at $30 \mathrm{~min}$ post-injection of M6P-GFLG-HPMA-GFLG- ${ }^{32} \mathrm{P}-\mathrm{TFO}, \mathrm{HPMA}-\mathrm{GFLG}-{ }^{32} \mathrm{P}-\mathrm{TFO}$ or ${ }^{32} \mathrm{P}-\mathrm{TFO}$ at dose of $0.2 \mathrm{mg} \mathrm{TFO} / \mathrm{kg}$ of body weight. The associated radioactivity was measured. The contribution of each liver cell type was exposed as percentage of total liver uptake. Results are expressed as the mean \pm SD $(n=3)$. 
A

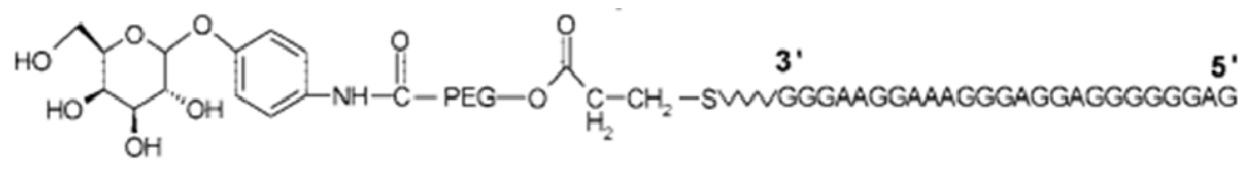

B

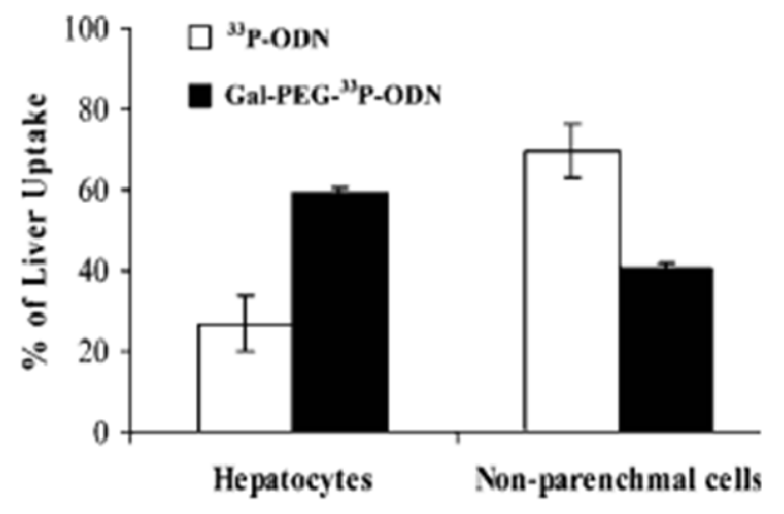

Figure 2-6 Delivery efficiency of Gal-PEG- ${ }^{33} \mathrm{P}-\mathrm{ODN}$. A) Structure of Gal-PEG- ${ }^{33} \mathrm{P}-\mathrm{ODN}$. B) Intrahepatic distribution of ${ }^{33} \mathrm{P}-\mathrm{ODN}$ and Gal-PEG- ${ }^{33} \mathrm{P}-\mathrm{ODNs}$ after systemic administration in rats. Liver cells were isolated at 30 min post-injection of ${ }^{33} \mathrm{P}-\mathrm{ODN}$ or Gal-PEG- ${ }^{33} \mathrm{P}-\mathrm{ODN}$ by liver perfusion. The associated radioactivity was measured. Results are expressed as the mean $\pm \mathrm{SD}(\mathrm{n}=4)$. 
only significantly higher cellular uptake but also enhanced gene silencing compared to the un-conjugated siRNA. Following intravenous injection into mice Chol-siRNAs are taken up by several tissues, including the liver, jejunum, heart, kidneys, lungs and fat tissue. Significant silencing of apoliporotein B $(\mathrm{apoB})$ gene was observed at mRNA and protein levels in the liver and the jejunum. Furthermore, this reduction resulted in a decreased plasma apoB protein level and consequent decreased level of blood cholesterol. However, the siRNA dose $(50 \mathrm{mg} / \mathrm{kg})$ in animal experiments is too high for clinical applications. Cholesterol conjugate was also applied to deliver ODNs. ${ }^{177}$ Cheng et al. conjugated cholesterol to ODNs by a disulfide bond and showed high cellular uptake, because the cholesterol conjugation increases hydrophobicity and cellular association.

In addition to Chol-siRNA conjugate, a series of siRNAs have been conjugated with lipid like carriers, including $\alpha$-tocopherol (vitamin E), steroid and lipids. ${ }^{146,178,179}$ Lipoproteins may facilitate the cellular uptake of these conjugates. A critical factor determining the affinity of fatty acid-conjugated siRNAs to lipoprotein particles is the length of the alkyl chain, a major determinant of lipophilicity. ${ }^{146}$ So far, only lipophilic siRNAs showed lipid-metabolism-related-gene silencing, Apo B. Therefore, here rises a question. Does the lipid-like-molecule-siRNA conjugate only silence lipid metabolism related genes? More research is needed to clarify this question.

\subsection{Pharmacokinetics and Biodistribution}

ODNs are accumulated in most peripheral tissues after systemic administration, particularly kidney and liver, but little distribution to the central nervous system. The biphasic plasma half-lives of ODNs are several minutes, while phosphorothioate ODNs showed distribution half-lives ranging from many minutes to hours. ${ }^{180-183}$ The major route of ODN elimination is the kidneys, even though phosphorothioate ODNs efficiently bind to plasma proteins. This highly protein bound ODNs usually have a longer circulation time than would be expected of a simple phosphodiester ODNs.

Pharmacokinetics profiles of various chemically modified ODNs, especially for the 2'-MEO AS-ODNs, has been determined and found to be similar to those of phosphorothioate ODNs. ${ }^{182,184}$ The in vivo fate of 2'-MEO modified ODNs were also studied and compared in rodents, monkeys and humans. ${ }^{185}$ In this study, plasma pharmacokinetics of 2'-MOE partially modified AS-ODNs was similar in mice, rats, dogs, monkeys, and humans. After intravenous administration, plasma concentration-time profiles are polyphasic as characterized by a rapid distribution phase (half-lives in hours), and followed by slower elimination phase with half-lives, but longer in the study of humans, from 5 to 31 days. The plasma clearance of monkeys and humans was similar, about one tenth of the mice. Allometric comparison of clearance estimated at similar doses across all species was done. From mouse to man, there showed a linear relationship based on body weight alone.

The pharmacokinetic profile of LNA ODNs in rodents is similar to that of PS ODNs, except that there was high urinary excretion of intact LNA ODNs compared to PS 
ODNs. ${ }^{186}$ This is possibly due to extensive binding of PS ODNs to serum proteins leading to poor renal clearance, while LNA ODNs do not bind to serum proteins and thus are easily filtered out of the kidney. ${ }^{187}$ Even though this property of LNA ODNs reduces non-specific interaction, it also makes the clearance of LNA ODNs faster. Furthermore, chimeric DNA/LNA ODNs are more stable than isosequential PS ODNs, which have half-lives of more than 10 hours. Peptide nucleic acids (PNAs) did not show any increase in the distribution half-life. ${ }^{188}$

Native siRNAs had an elimination half-life of 6 min only, ${ }^{176}$ shorter than that of ODNs. The shorter half-life may be partly due to the higher instability of siRNAs compared to ODNs. The biodistribution of radiolabeled siRNAs in mice showed an accumulation primarily in the liver and kidneys, which is similar to that of ODNs. ${ }^{189}$ They were also detected in the heart, spleen and lung. Actually, the high renal uptake facilitates the target delivery of siRNA to this tissue. ${ }^{190}$

Conjugation of cholesterol, ${ }^{176}$ tocopherol,,${ }^{179}$ or other lipid moieties, ${ }^{191}$ enhances the binding of ODNs to serum lipoproteins and/or albumin. This results in enhanced circulation time and, more importantly, hepatic uptake via the low-density lipoprotein receptor. Other conjugation with macro molecular materials also changed the pharmacokinetic profiles of ODNs and siRNAs.

Bioimaging allows real time analysis of ODN and siRNA. ${ }^{192}$ Micro SPECT or other radioimaging techniques can provide detailed information on the distribution of ODNs and siRNAs. However, there are several underlying issues. One is how to separate the label from the molecule being studied, which is a common problem for almost all radiolabeling methods. More importantly, there may be a discrepancy between physical biodistribution and functional biodistribution of ODNs and siRNAs. For example, in a study, LNA ODNs were designed to cause an alteration in mRNA splicing. The major effects were observed in liver, colon, and small intestine; however the major site of accumulation of the LNA was the kidney. ${ }^{193}$ Therefore, one should be carefully in predicting pharmacological effects when using radiolabeling data although it represents the pharmacokinetics and biodistribution of gene drugs.

\subsection{Clinical Trials}

Several companies initiated clinical trials of ODNs in the early 1990s. The most intensively studied ODNs are phosphorothioate ODNs, which are well absorbed and distributed widely to most peripheral tissues, but poorly distributed to the brain. ${ }^{194}$ Other modified ODNs also proceeded to clinical trials. Table 2-1 shows a universal applicability of antisense strategies to treat a broad range of diseases including viral infections, cancer and inflammatory diseases. In 1998, the first antisense drug Vitravene (Fomivirsen) was approved by the FDA for treating cytomegalovirus (CMV) induced retinitis in patients with AIDS. ${ }^{58}$ However, it is the only ODN drug approved by the FDA so far, even though several PS ODNs have been in Phase III trials, such as Affinitac (ISIS 3521) and Alicaforsen (ISIS 2302). However, Alicaforsen failed to show significant 
Table 2-1 Current clinical trials for oligonucleotides and siRNA.

\begin{tabular}{|c|c|c|c|}
\hline Products & Nucleic acids & Disease & Status \\
\hline Genasense & AS-ODN & Cancer & Phase II/III \\
\hline AP 12009 & AS-ODN & Astrocytoma, glioblastoma & Phase IIb/III \\
\hline AEG35156 & AS-ODN & Cancer & Phase I/II \\
\hline OGX-427 & AS-ODN & Bladder neoplasm & Phase I \\
\hline SPC2996 & AS-ODN & Chronic lymphocytic leukaemia & Phase I/II \\
\hline G4460 & AS-ODN & Chronic myelogenous leukemia & Phase II \\
\hline Alicaforsen (ISIS) & AS-ODN & Crohn's disease & Phase III \\
\hline Angiozyme & Ribozyme & Matastatic colorectal cancer & Phase II \\
\hline Herzyme & Ribozyme & Cancer & Phase I \\
\hline Angiozyme & Aptamer & Cancer & Phase II \\
\hline Herzyme & Aptamer & Cancer & Phase I \\
\hline AGN211745 & siRNA & AMD & Phase II \\
\hline DOTAP:Chol-fus1 & siRNA & Non-small-cell lung cancer & Phase I \\
\hline I5NP & siRNA & AKI & Phase I \\
\hline Cand5 & siRNA & Diabetic macular edema & Phase II \\
\hline AVI-4658 (PMO) & Other & Becker's muscular dystrophy & Phase I/II \\
\hline
\end{tabular}


efficacy in a Phase III study for treating Crohn's disease ${ }^{195}$ and is now being investigated in a restructured Phase III trial. Many other ODNs have reached the stage of clinical trials. ISIS 104838 against tumor necrosis factor- $\alpha(\mathrm{TNF}-\alpha)$ is being tested for treating inflammatory diseases such as rheumatoid arthritis and psoriasis. ${ }^{184}$

A retrovirally expressed ribozyme that targets the HIV tat and rev exons, entered clinical testing in late 1996 and is currently in phase II testing for patients with AIDS-related lymphoma. Ribozyme Pharmaceuticals (Boulder, CO, USA) performs clinical trials on ANGIOZYME (Table 2-1). ANGIOZYME is a stabilized hammerhead ribozyme that is targeted against the vascular endothelial growth factor (VEGF) receptor. It is designed to reduce tumor growth by inhibiting angiogenesis. The third, HEPTAZYME, a ribozyme targeting the 5'-untranslated region (5'-UTR) of the hepatitis $\mathrm{C}$ virus (HCV) RNA genome, has recently completed a phase I/II clinical trial in patients with chronic hepatitis $\mathrm{C}$.

Acuity Pharmaceuticals performed the first clinical trial for siRNA therapy to Age-related Macular Degeneration (AMD) in 2004. After the successful Phase II trials reported that all doses were well tolerated without adverse systemic effects, testing has now moved into Phase III trials. The siRNA treatment for AMD was also performed by Allergan to Phase II trial. The trials related to various diseases, such as solid tumor cancer and acute kidney injury (AKI), are in good progress. The active trials so far are listed in the Table 2-1. However, another interesting report about a Phase II clinical trial by OPKO Health on the treatment of diabetic macular edema, which is swelling of the retina in diabetes mellitus due to leakage of fluid from blood vessels within the macula. It was shown that anti-VEGF siRNA efficacy in the eye is not due to specific gene silencing but because of nonspecific stimulation of the TLR3 pathway, ${ }^{196}$ which can reduce angiogenesis, but the therapeutic effects observed in other applications of siRNA are still encouraging. 


\section{CHAPTER 3. HPMA POLYMER-BASED SITE-SPECIFIC DELIVERY OF OLIGONUCLEOTIDES TO HEPATIC STELLATE CELLS}

\subsection{Introduction}

Chronic liver injury and inflammation of hepatocytes may lead to overproduction of type I collagen and other extracellular matrix (ECM) by hepatic stellate cells (HSCs), which are distributed throughout the hepatic lobule and serve as the principle fibrogenic cells. ${ }^{197}$ Activation of HSCs affects liver architecture and eventually liver function. ${ }^{198}$ Until now, no pharmaceutical intervention is available to treat this fibrotic disease. ${ }^{199}$ The application of most antifibrotic drugs has not been successful, partly because these drugs do not accumulate in the target liver cells or cause serious side effects elsewhere in the body. Alteration of the pharmacokinetic profiles of antifibrotic drugs by means of drug targeting represents a promising approach in the development of an effective antifibrotic drug. $^{200}$

Direct inhibition of type I collagen synthesis by HSCs is a potential target to prevent liver fibrosis. Earlier, we developed a triplex-forming oligonucleotide (TFO), which can form a triplex with the target sequence (C1) located in the rat $\alpha 1(\mathrm{I})$ collagen gene promoter and inhibit the transcription of this gene. ${ }^{34}$ Mannose-6-phosphate/insulin like growth factor II (M6P/IGFII) receptor is expressed on HSCs, and its expression is up-regulated upon activation of these cells due to acute or chronic liver injury. ${ }^{201}$ Therefore, this TFO molecule is a potential candidate for treating liver fibrosis.

Following systemic administration, oligonucleotides (ODNs) widely distribute throughout the body with higher accumulation in the liver and kidney. ${ }^{202-204} \mathrm{We}$ determined the in vivo distribution of the TFO molecules in normal and fibrotic rats. ${ }^{38}$ Almost $45 \%$ of the injected dose was accumulated in the liver at $30 \mathrm{~min}$ post tail vein injection in normal rats, but only $35 \%$ of injected dose in fibrotic rats. Since the intrahepatic distribution of the TFO was non-specific, we synthesized mannose-6-phosphate-bovine serum albumin (M6P-BSA) and conjugated to TFO via a disulfide bond for its enhanced delivery to HSCs. ${ }^{41}$ Since the treatment of liver fibrosis may require repeated injections of TFO at high dose, high molecular weight globular BSA (66430 Da) may not be a suitable carrier for TFO delivery to the HSCs due to possible immune reaction. N-(2-hydroxypropyl) methacrylamide (HPMA) copolymer has shown great potential for delivery of small molecular drugs. ${ }^{205-207}$ Although HPMA has also been used for oligonucleotide delivery, no targeting ligands were used for its sitespecific delivery to target cells and its biodistribution and uptake by different liver cell types was not determined after systemic administration. Even though biodistribution of oligonucleotides to the liver has been reported before, ${ }^{208-210}$ the authors did not determine oligonucleotides delivery to HSCs, which are the principal liver fibrotic cells.

In this chapter, we conjugated M6P to HPMA and then to TFO via GFLG linker, which is known to be cleaved by lysosomal enzymes. ${ }^{208}$ Following bioconjugation and purification, we determined i) whether TFO can be released from the conjugate after 
cellular uptake, ii) biodistribution of M6P-GFLG-HPMA-GFLG- ${ }^{32} \mathrm{P}-\mathrm{TFO}$ at the whole body, organ (liver) and cellular (liver cells) levels after tail vein injection into rats.

\subsection{Experimental Procedures}

\subsubsection{Materials}

Poly (HPMA-co-GFLG-ONP) was purchased from Varian Inc (Amherst, MA). p-Nitrophenyl- $\alpha$-D-mannopyranoside (pnpM), phosphorous oxide chloride, palladium (10 wt $\%$ on activated carbon), papain, methylene blue, N,N'-diisopropylethylamine, Histodenz (nycodenz) and pronase were purchased from Sigma-Aldrich (St. Louis, MO). Sephadex G75 (superfine) was procured from Pharmacia Fine Chemicals AB (Uppsala, Sweden). Dialysis tubing (molecular weight cutoff of $1000 \mathrm{Da}$ ) was purchased from Spectrum Laboratories, Inc. (Houston, TX). BioGel P-6 DG Gel was from Bio-Rad Laboratories (Hercules, CA). $\left[\gamma^{32} \mathrm{P}\right]$-dATP was purchased from MP Biomedicals (Irvine, $\mathrm{CA}$ ), and T4 polynucleotide kinase was from New England Biolabs (Beverly, MA). Soluene-350 (tissue solubilizer) and HionicFluor (scintillation fluid) were purchased from Perkin-Elmer (Boston, MA). Hydrogen peroxide $\left(\mathrm{H}_{2} \mathrm{O}_{2}\right)$ was purchased from Fisher Chemical (Fair Lawn, NJ). Heparin was purchased from American Pharmaceutical Partners, Inc. (Los Angeles, CA). $\mathrm{Ca}^{2+} / \mathrm{Mg}^{2+}$-free Hank's balanced salt solution (Cellgro) was purchased from MediaTech (Washington, DC), and type IV collagenase was from Worthington Biochemical Corporation (Lakewood, NJ). Isoflurane was purchased from Baxter Pharmaceutical Products, Inc. (Deerfield, IL). TFO, which was a 25 mer antiparallel fully phosphorothioate ODN (3'-GAGGGGGGAGGAGGGAAAGGAAGGG-5') targeting rat $\alpha 1$ (I) collagen gene promoter, and TFO-3'-NH2 were synthesized by Invitrogen (Carlsbad, CA). All solvents and chemicals used in this study were used as available without further purification.

\subsubsection{Animals}

Male Sprague-Dawley rats weighing 130-150 g were purchased from Harlan Co. (San Diego, CA) and were housed individually under the controlled light (12/12 h) and temperature conditions and had free access to food and water.

\subsubsection{Synthesis of p-Isothiocyanatophenyl-6-phospho- $\alpha-D$-mannopyranoside}

p-nitrophenyl- $\alpha$-D-mannopyranoside (pnpM) $(3 \mathrm{~g}, 10 \mathrm{mmol})$ was dissolved in pyridine ( $4 \mathrm{~mL}, 50 \mathrm{mmol})$, acetonitrile $(10 \mathrm{~mL}, 190 \mathrm{mmol})$, and water $(0.4 \mathrm{~mL}, 22$ mmol). To this solution, phosphorus oxide chloride ( $4 \mathrm{~mL}, 44 \mathrm{mmol}$ ) was added, and the mixture was stirred for $1 \mathrm{~h}$ at $0^{\circ} \mathrm{C}$. The reaction mixture was poured onto $120 \mathrm{~g}$ of ice. $\mathrm{pH}$ was adjusted to 7.0 by slowly adding $2.5 \mathrm{M} \mathrm{NaOH}$ on ice and the neutralized solution was evaporated to dryness. The solid material was dissolved in $150 \mathrm{~mL}$ water. The 
solution was concentrated under reduced pressure at $35^{\circ} \mathrm{C}$ in a rotary evaporator to a final volume of 3-4 mL. The concentrated solution was kept at $4^{\circ} \mathrm{C}$ overnight for crystallization and crystals was filtered and washed with $10 \mathrm{~mL}$ absolute ethanol. The compound was recrystallized from a $10 \mathrm{~mL}$ water/100 $\mathrm{mL}$ ethanol mixture, redissolved in water, and lyophilized to give p-nitrophenyl-6-phospho- $\alpha$-D-mannopyranoside (pnpM6P). pnpM6P (1 mmol) was dissolved in $20 \mathrm{~mL}$ of a 4:1 (v/v) methanol-water mixture. To this solution, $30 \mathrm{mg}$ of $10 \%$ palladium on activated carbon was added. The suspension was stirred under hydrogen $(1 \mathrm{~atm})$ at room temperature for two hours. After filtration, the methanolic solution was evaporated under reduced pressure at $40^{\circ} \mathrm{C}$ and lyophilized to give p-aminophenyl-6-phospho- $\alpha$-D-mannopyranoside (papM6P).

\subsubsection{Synthesis of M6P-GFLG-HPMA-GFLG-TFO Conjugate}

The synthesis scheme of M6P-GFLG-HPMA-GFLG-TFO is shown in Figure 3-1. Poly(HPMA-co-MA-GFLG-ONP) (5 mg, $2.25 \mu \mathrm{mol}$ of ONP) and TFO-NH2 (0.8 mg, 0.1 $\mu \mathrm{mol})$ were dissolved in anhydrous dimethyl sulfoxide (DMSO, $100 \mu \mathrm{L}$ ). Then, $\mathrm{N}, \mathrm{N}$-diisopropylethylamine (DIPEA, $3 \mu \mathrm{L}$ ) was added and reacted overnight at room temperature. papM6P (3 mg, $10 \mu \mathrm{mol})$ was dissolved in $\mathrm{H}_{2} \mathrm{O}(100 \mu \mathrm{L})$ and dimethyl sulfoxide (DMSO) $(200 \mu \mathrm{L})$ mixture, and DIPEA $(3 \mu \mathrm{L})$ was added. Reaction was allowed to proceed for $24 \mathrm{~h}$ at room temperature. The reaction mixture was dialyzed against $\mathrm{H}_{2} \mathrm{O}$ for a day, purified on a Sephadex $\mathrm{G} 75$ Gel column with pure water, freeze-dried, kept in $-80^{\circ} \mathrm{C}$ and resuspended in $0.9 \%$ saline before use.

\subsubsection{In Vitro Characterization}

The purity of intermediate and final products were monitored by reverse phase-high performance liquid chromatography (RP-HPLC), which was carried on Altech Prosphere C18 column $(250 \mathrm{~mm} \times 4.6 \mathrm{~mm} \times 5 \mu \mathrm{m})$ by Waters Breeze HPLC/GPC system (Waters, Milford, MA) with detection at $260 \mathrm{~nm}$ using a gradient of $40 \%$ to $100 \%$ acetonitrile in $0.1 \mathrm{M}$ triethylammonium acetate (TEAA) at a flow rate of $1 \mathrm{~mL} / \mathrm{min}$ at room temperature. M6P-GFLG-HPMA-GFLG- ${ }^{32} \mathrm{P}-\mathrm{TFO}$ was analyzed by $20 \%$ polyacrylamide gel electrophoresis (PAGE) at $80 \mathrm{~V}$ for $2 \mathrm{~h}$. The gels were stained with methylene blue or autoradiographed.

The molecular weight of M6P-GFLG-HPMA-GFLG-TFO was also measured by gel permeation chromatography (GPC), which was carried on Waters Ultrahydrogel 250 column $(7.8 \times 300 \mathrm{~mm})$ by Waters Breeze HPLC/GPC system (Waters, Milford, MA) with Waters 2414 Refractive Index detector and mobile phase containing $0.02 \%$ sodium azide $\left(\mathrm{NaN}_{3}\right)$ in HPLC water, at a flow rate of $0.5 \mathrm{~mL} / \mathrm{min}$. Polyethylene oxide (PEO) of different molecular weights (MW=20000, 31380, 50450 and $71700 \mathrm{Da}$ ) was used as standards for calibration. HPMA-co-MA-ONP was used as a positive control and the molecular weight of M6P-GFLG-HPMA-GFLG-TFO was determined. 


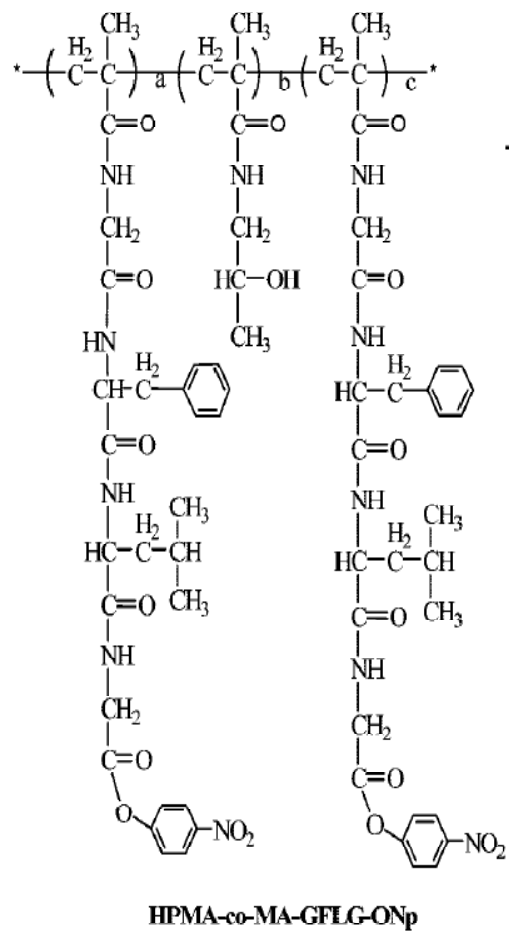

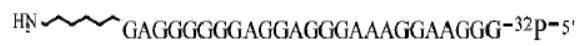

(i)
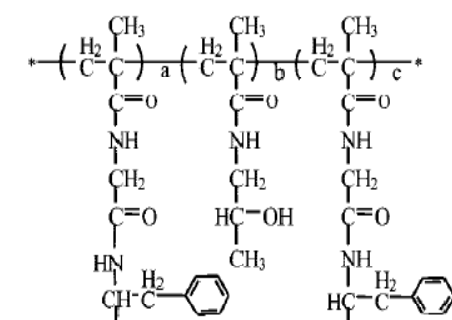

$\mathrm{C}=0 \quad \mathrm{C}=0$

管 $\mathrm{H}_{2} \mathrm{CH}_{2} \mathrm{CH}_{3}$

$\mathrm{NH}$

$\mathrm{CH}_{2}$

$C=0$

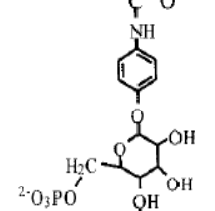

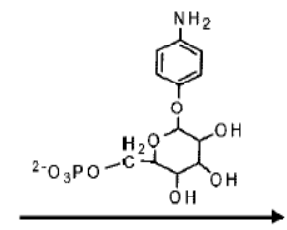

(ii)

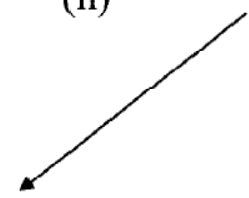

Figure 3-1 Synthesis scheme of M6P-GFLG-HPMA-GFLG-TFO. 


\subsubsection{Determination of Sugar Content}

Sugar content was determined by the resorcinol-sulfuric acid method. ${ }^{211}$ Two hundred microliters of $6 \mathrm{mg} / \mathrm{mL}$ resorcinol and $1 \mathrm{~mL}$ of $75 \%$ sulfuric acid were added to M6P-GFLG-HPMA-GFLG-TFO solution containing 5-100 nmol of sugar in $200 \mu \mathrm{L}$. The mixtures were vortexed and heated at $90^{\circ} \mathrm{C}$ for $30 \mathrm{~min}$ and subsequently placed in a cold water bath for $30 \mathrm{~min}$ in the dark. The optical density of the solution was measured at 430 $\mathrm{nm}$. PnpM was used to generate the standard curve to calculate the number of M6P per conjugate.

\subsubsection{In Vitro Enzymatic Dissociation and Triplex Formation of M6P-GFLG-HPMA-GFLG- ${ }^{32} P-T F O$}

To determine whether TFO will be dissociated from M6P-GFLG-HPMA-GFLG-TFO conjugate after cellular uptake, papain $(10 \mu \mathrm{M})$, glutathione (GSH, $250 \mathrm{mM})$ and M6P-GFLG-HPMA-GFLG- ${ }^{32}$ P-TFO (TFO concentration $100 \mu \mathrm{M}$ ) were incubated together at $37^{\circ} \mathrm{C}$ in $0.1 \mathrm{M}$ phosphate buffer containing $1 \mathrm{mM}$ EDTA (PE buffer, total volume of $300 \mu \mathrm{L}$ ). At 1, 2, 8 and $24 \mathrm{~h}, 100 \mu \mathrm{L}$ of the sample was collected and analyzed by $20 \%$ PAGE at $80 \mathrm{~V}$ for $2 \mathrm{~h}$, followed by autoradiography.

To determine whether the TFO released from M6P-GFLG-HPMA-GFLG-TFO can still form triplex, the target duplex DNA was prepared by equal amounts of ODNs, T1: 5'-GAGGGGGGAGGAGGGAAAGGAAGGGAAAGG-3' and T2:

5'-CCTTTCССТTCСТTТCССТССТССССССТC-3', being heated at $80^{\circ} \mathrm{C}$ for $5 \mathrm{~min}$ in $0.25 \mathrm{M} \mathrm{NaCl}$, followed by slow cooling to room temperature. Triplex formation was initiated by the mixing of $3 \mu \mathrm{L}$ of $3 \times$ buffer $(135 \mathrm{mM}$ Tris-acetate, $\mathrm{pH} 7.0,30 \mathrm{mM}$ $\left.\mathrm{MgCl}_{2}\right), 3.5 \mu \mathrm{L}$ of duplex DNA $(\sim 3 \mu \mathrm{g})$, and $2.5 \mu \mathrm{L}$ of the released ${ }^{32} \mathrm{P}-\mathrm{TFO}\left(\sim 1 \times 10^{5}\right.$ $\mathrm{cpm})$. The reaction mixture was incubated at $37^{\circ} \mathrm{C}$ for another $24 \mathrm{~h}$. Two microliters of a $50 \%$ glycerol solution containing bromophenol blue was added, and samples were directly loaded onto $15 \%$ native polyacrylamide gel, prepared in a buffer containing 50 $\mathrm{mM}$ Tris-acetate, $\mathrm{pH} 7.0$, and $10 \mathrm{mM} \mathrm{MgCl}_{2}$. Electrophoresis was performed at $8 \mathrm{~V} / \mathrm{cm}$ at $4^{\circ} \mathrm{C}$ in the buffer containing $89 \mathrm{mM}$ Tris-Borate and $20 \mathrm{mM} \mathrm{MgCl}_{2}$. The gel was autoradiographed at $4^{\circ} \mathrm{C}$ overnight.

\subsubsection{Transfection of TFO and M6P-GFLG-HPMA-GFLG-TFO}

Immortalized rat hepatic stellate cells (HSC-T6) kindly provided by Dr Scott Friedman of Mount Sinai School of Medicine, New York were seeded in 6-well plates at a density of $11 \times 10^{5}$ cells $12 \mathrm{~h}$ until $50 \%$ confluence in DMEM containing $10 \%$ of FBS. The growth medium was replaced with a pre-warmed serum-free DMEM medium. TFO was mixed with pyridinium cationic liposome at 3/1 (+/-) charge ratio and then used for transfection at the dose of $5 \mu \mathrm{g}$ TFO/well. For the conjugate,

M6P-GFLG-HPMA-GFLG-TFO was dissolved in saline and applied to the cells at the dose of $5 \mu \mathrm{g}$ TFO. Negative control wells were added the same amount 
M6P-GFLG-HPMA. Six hours later 10\% FBS was added to each well, and then the cells were cultured for additional $72 \mathrm{~h}$. Following transfection, the cell culture medium was concentrated using Microcon YM-30 columns (Millipore) to retain proteins of $>30 \mathrm{kDa}$. The concentrated medium was lysed using $2 \times$ Laemmli sodium dodecyl sulfate (SDS) sample buffer containing $100 \mathrm{mM}$ Tris, $\mathrm{pH}$ 6.8, $200 \mathrm{mM}$ dithiothreitol (DTT), 4\% SDS, $20 \%$ glycerol and $0.2 \%$ bromophenol blue. To detect $\beta$-actin which was used as an intrinsic reference of sample loading, the cells were lysed directly with $1 \times$ Laemmli SDS sample buffer. The lysate samples were boiled at $100^{\circ} \mathrm{C}$ for $5 \mathrm{~min}$ and subjected to $4 \%$ to 15\% SDS-polyacrylamide (SDS-PAGE) gel electrophoresis and subsequently transferred to Immobilon polyvinylidene fluoride (PVDF) membrane (Millipore). After blocking with $5 \%$ non fat dried milk in $1 \times$ PBST containing $0.05 \%$ Tween-20 in PBS for $1 \mathrm{~h}$ at room temperature, the membrane was incubated with goat anti-rat type I collagen and anti-actin primary antibodies (Santa Cruz) for $16 \mathrm{~h}$ at $4^{\circ} \mathrm{C}$. The membranes were then incubated with horseradish peroxidase (HRP)-conjugated donkey anti-goat secondary antibody (Santa Cruz) for $1 \mathrm{~h}$ at room temperature. Target proteins were detected by enhanced chemiluminescence (ECL, GE Healthcare).

\subsubsection{Biodistribution of M6P-GFLG-HPMA-GFLG- ${ }^{32} P-T F O$}

The animal protocol was approved by the Animal Care and Use Committee (ACUC), Department of Comparative Medicine, University of Tennessee Health Science Center, Memphis, TN 38163. Male Sprague-Dawley rats weighing 130-150 g were used in this study and three rats were used for each time point. Unlabeled and M6P-GFLG-HPMA-GFLG- ${ }^{32} \mathrm{P}-\mathrm{TFO}$ were mixed in saline to give a final concentration of $1 \mathrm{mg} / \mathrm{mL}$ and specific activity of $1 \times 10^{6} \mathrm{cpm} / \mathrm{mL}$. Rats were anesthetized by inhalation of isoflurane, and M6P-GFLG-HPMA-GFLG- ${ }^{32} \mathrm{P}-\mathrm{TFO}$ was injected via tail vein at a dose of $0.2 \mathrm{mg} / \mathrm{kg}$ body weight. At 2.5, 5, 15,30, 60 and $90 \mathrm{~min}$ postinjection, $0.5 \mathrm{~mL}$ blood was collected by cardiac puncture in heparinized tubes, and urine was collected directly from the bladder using a 0.26 gauge needle syringe. The animals were then sacrificed and major tissues (liver, kidney, spleen, heart, and lung) were collected, washed, blotted dry, weighed, and stored at $-80^{\circ} \mathrm{C}$. The radioactivity of the urine sample was counted directly after adding $10 \mathrm{~mL}$ of scintillation fluid. One hundred and fifty microliters of plasma and $150 \mathrm{mg}$ of each tissue were incubated with $2 \mathrm{~mL}$ tissue solubilizer for $3 \mathrm{~h}$ at $55^{\circ} \mathrm{C}$ and overnight at $37^{\circ} \mathrm{C}$ in a shaker. Four hundred microliters of $\mathrm{H}_{2} \mathrm{O}_{2}$ was added and incubated at $55^{\circ} \mathrm{C}$ for another $30 \mathrm{~min}$. Ten milliliters of scintillation fluid was added to each sample and the radioactivity was counted using a liquid scintillation counter.

\subsubsection{Determination of Pharmacokinetic Profiles}

Plasma data were analyzed using WinNonlin Professional (version 5.2, Pharsight Corporation, Mountain View, CA). M6P-GFLG-HPMA-GFLG- ${ }^{32} \mathrm{P}-\mathrm{TFO}$ plasma concentration data versus time were fitted into a two-compartment model. The tissue uptake clearance and index were calculated using the values up to 90 min after injection, assuming that TFOs were fairly stable within this period. 


\subsubsection{Induction of Liver Fibrosis}

Dimethylnitrosamine (DMN) induced liver fibrosis in rats has been shown to have a pathology closely resembling that of human cirrhosis. Male Sprague-Dawley rats weighing 130-150 g (Harlan Co., San Diego, CA) were housed individually under controlled light $(12 / 12 \mathrm{~h})$ and temperature conditions and had free access to food and water. To induce liver fibrosis, dimethyl nitrosamine (DMN) was injected intraperitoneally into rats at a dose of $10 \mathrm{mg} / \mathrm{kg}$ of body weight in saline. Injections were given in the afternoons of the first three consecutive days of each week for 4 weeks.

\subsubsection{Competition in Hepatic Uptake of M6P-BSA- ${ }^{33} P-T F O$}

Two minutes before the injection of M6P-GFLG-HPMA-GFLG- ${ }^{32} \mathrm{P}-\mathrm{TFO}$ at a TFO dose of $0.2 \mathrm{mg} / \mathrm{kg}$ (specific activity: $1 \times 10^{6} \mathrm{cpm} / \mathrm{mL}$ ), liver fibrotic rats received $10 \mathrm{mg} / \mathrm{kg}$

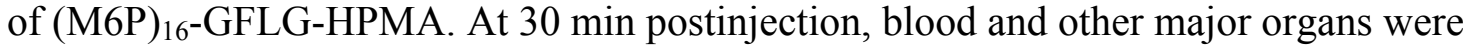
harvested as described above for radioactivity measurement.

\subsubsection{Isolated Rat Liver Perfusion}

To determine the effect of M6P on the hepatic uptake of TFOs by hepatocytes, HSCs, Kupffer and endothelial cells, the livers of normal and fibrotic rats were perfused in situ after intravenous administration of M6P-GFLG-HPMA-GFLG- ${ }^{32} \mathrm{P}-\mathrm{TFO}$, HPMA-GFLG- ${ }^{32} \mathrm{P}-\mathrm{TFO}$ or ${ }^{32} \mathrm{P}-\mathrm{TFO}$ at the dose of $0.2 \mathrm{mg}$ of TFO $/ \mathrm{kg}$ body weight. At 30 min post-administration, rats (200-250 g) were anesthetized by inhalation of isoflurane, $100 \mathrm{U}$ heparin was injected via the tail vein, the abdomen was opened, and the portal vein was cannulated with PE-60 polyethylene tube. The liver was first perfused with $2 \mathrm{~mL}$ of diluted heparin solution at 20 units $/ \mathrm{mL}$ to avoid blood clogs in the liver. The liver was pre-perfused in situ with $200 \mathrm{~mL}$ of $\mathrm{Ca}^{2+} / \mathrm{Mg}^{2+}$-free Hank's balanced salt solution at a flow rate of $15 \mathrm{~mL} / \mathrm{min}$ and was then perfused with Hank's balanced salt solution containing $0.05 \%$ type IV collagenase and $0.1 \%$ pronase for additional $250 \mathrm{~mL}$ at a flow rate of $10 \mathrm{~mL} / \mathrm{min}$. All the perfusion solutions were incubated at $37^{\circ} \mathrm{C}$. After perfusion, different liver cell types were separated and radioactivity was measured as described by Cheng et al. ${ }^{38}$ The contributions of various cell types to the total liver accumulation were calculated as the percentage of total hepatic uptake.

\subsubsection{Statistical Analysis}

Data were expressed as the mean \pm standard deviation (SD). The difference between any two groups was determined by ANOVA. $\mathrm{p}<0.05$ was considered statistically significant. 


\subsection{RESULTS}

\subsubsection{Synthesis and In Vitro Characterization of M6P-GFLG-HPMA-GFLG- ${ }^{32} P-T F O$}

The synthesis scheme of M6P-GFLG-HPMA-GFLG-TFO is shown in Figure 3-1. p-nitrophenyl- $\alpha$-D-mannopyranoside ( $\mathrm{pnpM}$ ) was phosphorylated to give $\mathrm{p}$-nitrophenyl 6-phospho- $\alpha$-D-mannopyranoside (pnpM6P). This intermediate product was characterized by electron spray ionization-mass spectrum (ES-MS) as described before (data not shown). ${ }^{41}$ RP-HPLC was used to monitor the purity of the intermediate and final products. From the RP-HPLC chromatograms of M6P, TFO, HPMA and M6P-GFLG-HPMA-GFLG-TFO (Figure 3-2), almost all free M6P and TFOs were removed from the conjugate after purification. M6P-GFLG-HPMA-GFLG- TFO was separated from free TFO using G75 column. As shown in Figure 3-2, M6P-GFLG-HPMA-GFLG- ${ }^{32} \mathrm{P}-\mathrm{TFO}$ conjugate was eluted earlier than ${ }^{32} \mathrm{P}-\mathrm{TFO}$. PAGE analysis of M6P-GFLG-HPMA-GFLG- ${ }^{32}$ P-TFO was also confirmed successful conjugation and purity of the conjugate. As shown in Figure 3-3, unlike free TFO, there was no band shift for the conjugate. We also measured the molecular weight of M6P-GFLG-HPMA-GFLG-TFO by gel permeation chromatography (GPC) and determined molecular weight was $51418 \mathrm{Da}$, which matched well with the calculated molecular weight by calculation of $51362 \mathrm{Da}$. Although poly(HPMA-co-GFLG-ONP) contains 8.33 mole $\%$ of ONP, the average number of M6P per conjugate was determined to be 14.67 .

\subsubsection{In Vitro Enzymatic Dissociation and Triplex Formation of M6P-GFLG-HPMA-GFLG- ${ }^{32} P-T F O$}

To determine whether TFO can be dissociated from M6P-GFLG-HPMA-GFLG ${ }^{32} \mathrm{P}-\mathrm{TFO}$ endocytosis in the lysosome, the radioactivity was measured after polyacrylamide gel electrophoresis and autoradiography at 1, 2, 8 and 24 $\mathrm{h}$ post-incubation of the conjugate with papain at $37^{\circ} \mathrm{C} .{ }^{212}$ The band of the conjugate gradually disappeared along the incubation time (Figure 3-4). In contrast, this conjugate resulted in no band shift in the absence of papain.

GFLG linker is expected to be stable in the bloodstream but cleaved inside the cells by enzymatic dissociation by lysosomal enzymes. ${ }^{213}$ The release from the M6P-GFLG-HPMA-GFLG-TFO conjugate will enable TFO to traffic to the nucleus for triplex formation with genomic DNA. There should be no adverse effect on triplex formation due to this conjugation and cleavage. The M6P-GFLG-HPMA-GFLG-TFO conjugate was treated with papain and incubated with the target duplex DNA for another $24 \mathrm{~h}$, and the sample was then applied on a $15 \%$ native polyacrylamide gel at $4^{\circ} \mathrm{C}$. Following electrophoresis, the gel was autoradiographed at $4^{\circ} \mathrm{C}$ overnight. As shown in Figure 3-5, there was triplex formation observed with target duplex DNA not only for free TFO (lane 2) but also for released TFO (lane 6), which was at a molar ratio of 200 between duplex DNA and TFO. 

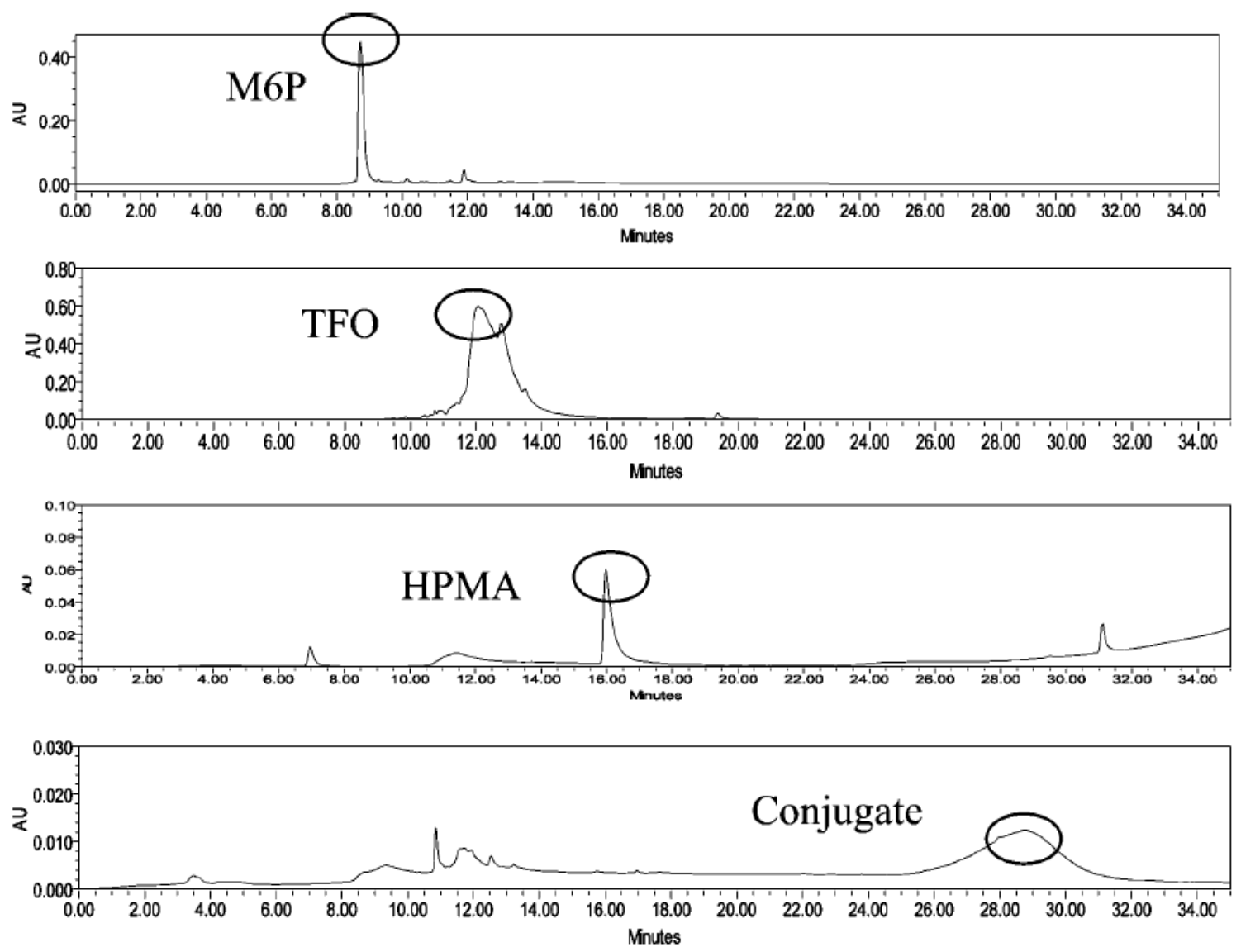

Figure 3-2 HPLC chromatography of M6P-GFLG-HPMA-GFLG-TFO conjugate. There is a new peak for conjugate different from the reactants, such as M6P, poly(HPMA-co-GFLG-ONP), and TFO. 


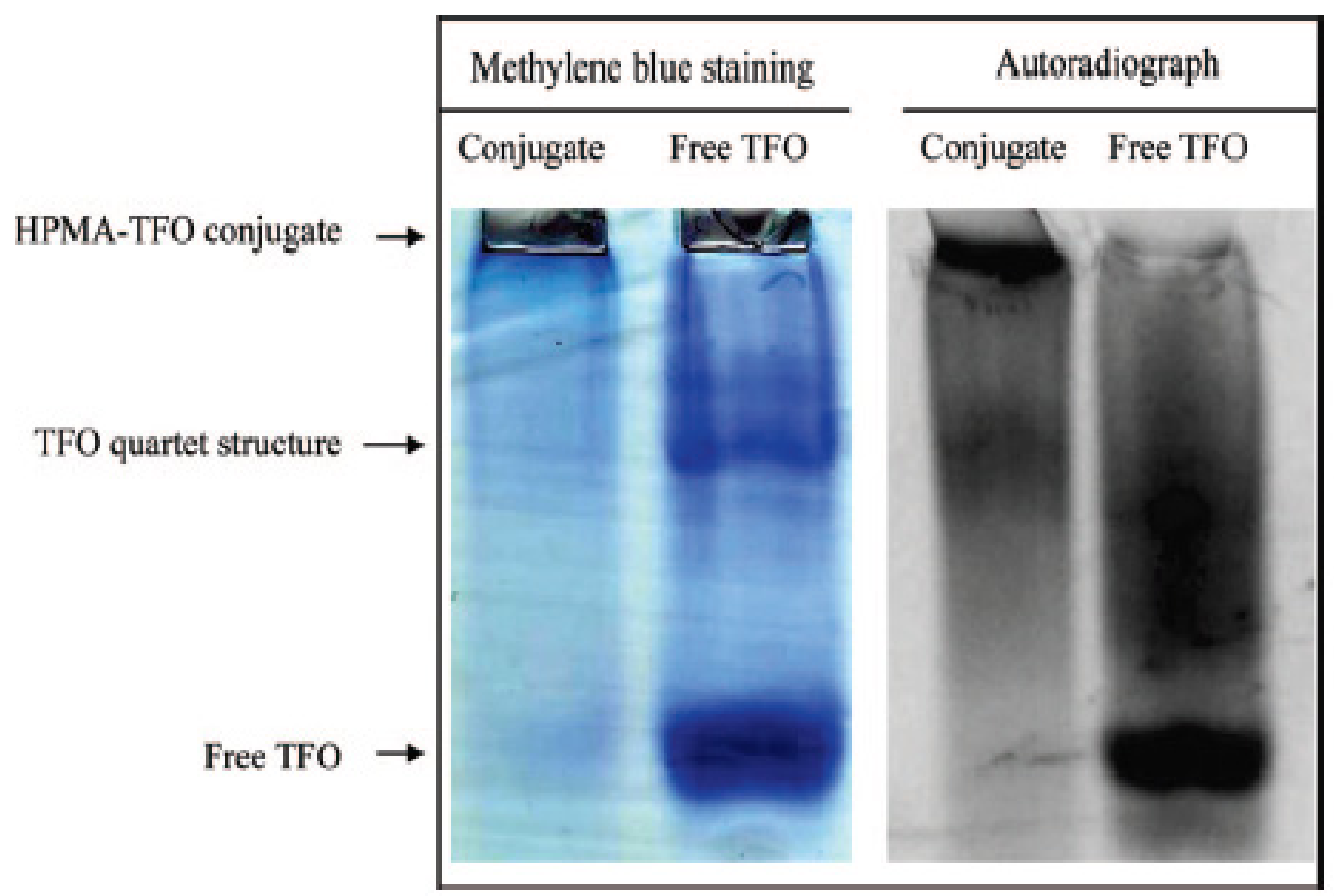

Figure 3-3 Polyacrylamide gel electrophoresis (PAGE) of M6P-GFLG-HPMA-GFLG-TFO. Methylene blue staining was on the left and autoradiography was on the right. There was no band shift for the conjugate. 


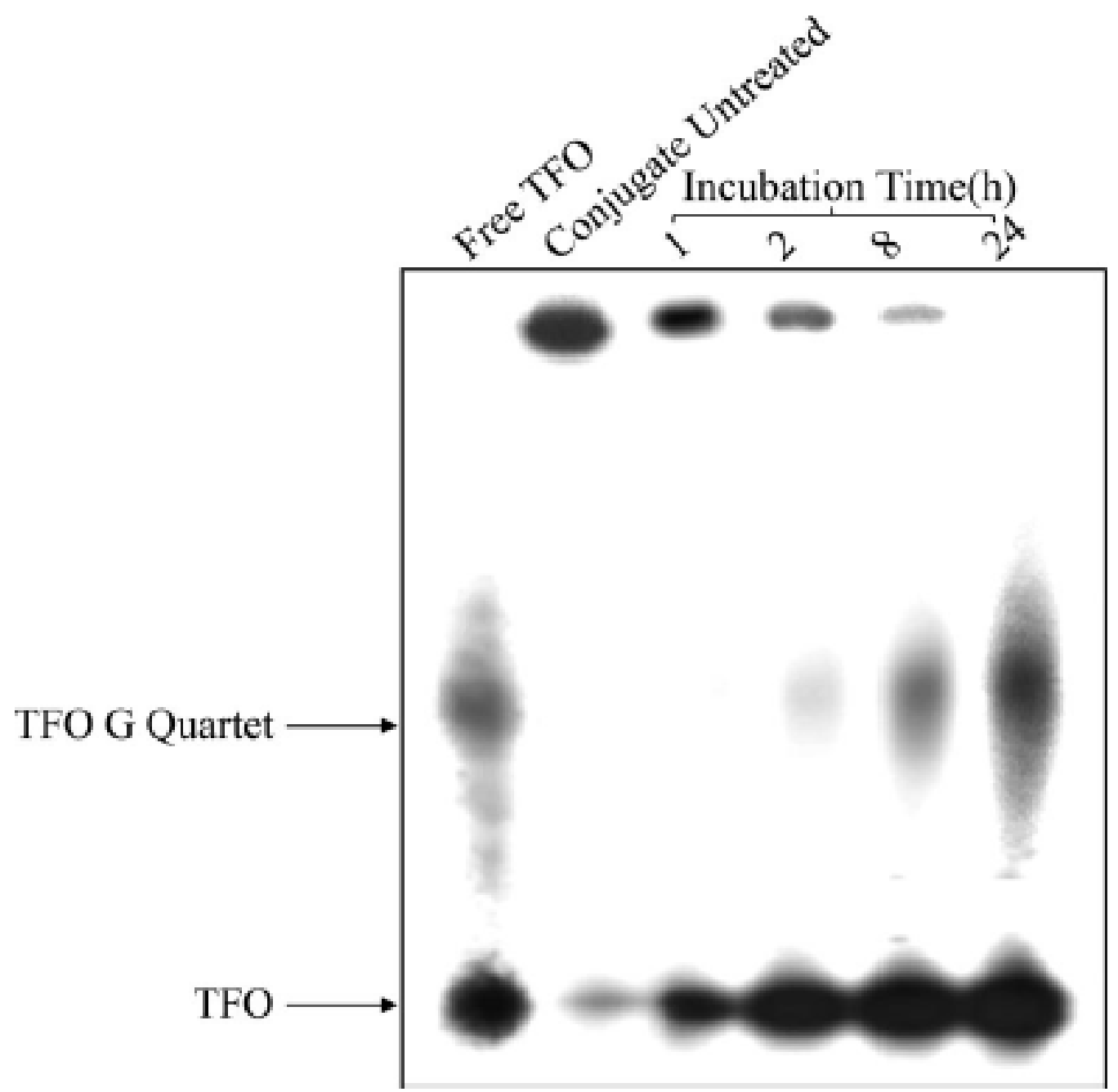

Figure 3-4 TFO dissociation from M6P-GFLG-HPMA-GFLG-TFO by papain. The conjugate was treated with papain for $1,2,8$, and $24 \mathrm{~h}$, followed by polyacrylamide gel electrophoresis (PAGE) and autoradiography. The conjugate not treated with papain was used as a control. 


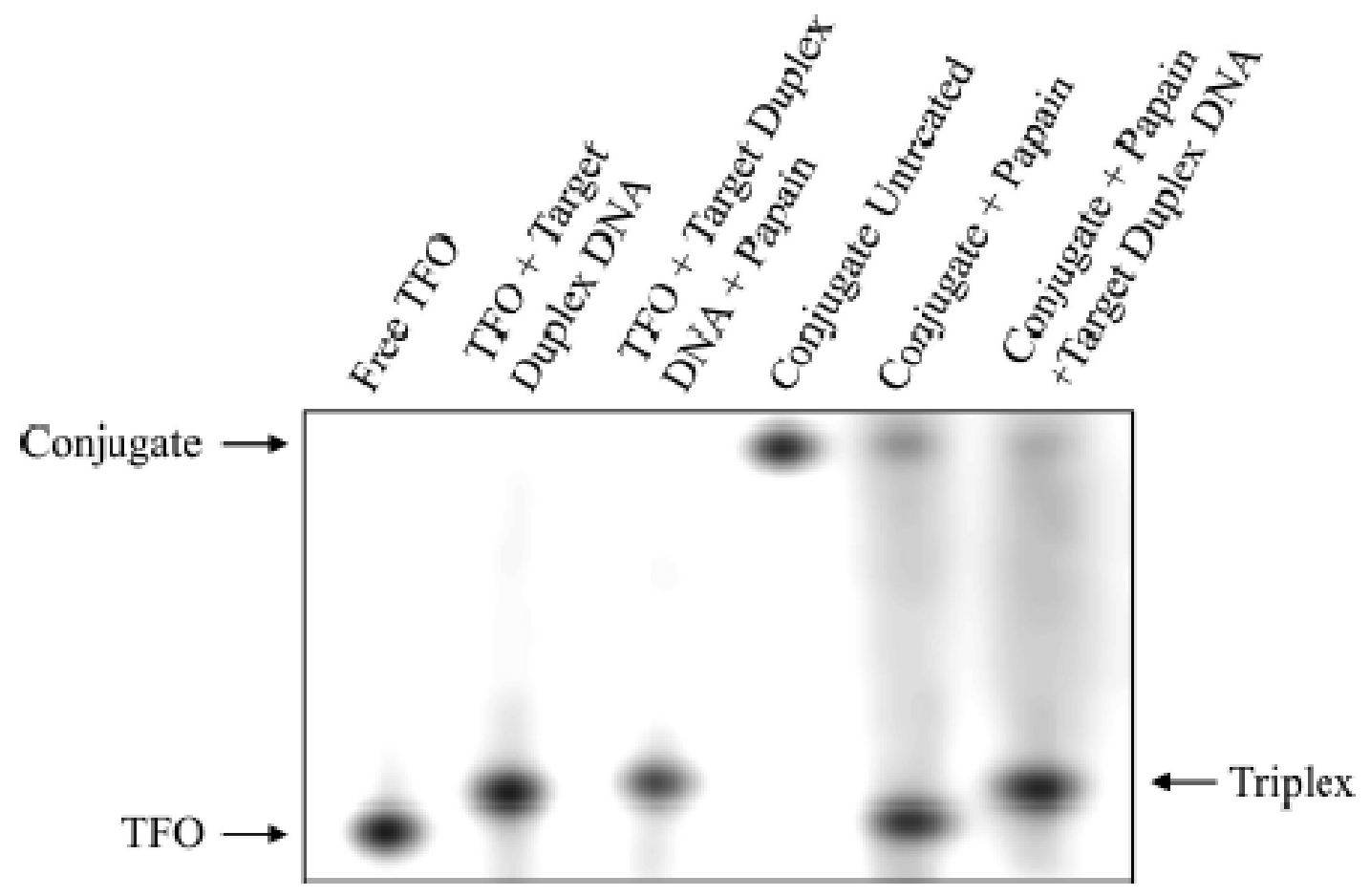

Figure 3-5 Triplex formation with target duplex DNA after release from M6P-GFLG-HPMA-GFLG- ${ }^{32}$ P-TFO conjugate. Samples were applied on $15 \%$ native PAGE at $4{ }^{\circ} \mathrm{C}$ in $89 \mathrm{mM}$ Tris-borate buffer, containing $\mathrm{MgCl}_{2}(20 \mathrm{mM})$ for $4 \mathrm{~h}$. Lane 1: ${ }^{32}$ P-TFO. Lane 2: duplex DNA/ ${ }^{32}$ PTFO (200:1) incubated for $24 \mathrm{~h}$. Lane 3: Duplex DNA $/{ }^{32}$ P-TFO (200: 1 ) and papain incubated for $24 \mathrm{~h}$. Lane 4: M6P-GFLG-HPMA-GFLG- ${ }^{32}$ P-TFO. Lane 5: M6P-GFLG-HPMA-GFLG- ${ }^{32} \mathrm{P}-\mathrm{TFO}$ incubated with papain for $24 \mathrm{~h}$ at $37^{\circ} \mathrm{C}$. Lane 6: duplex DNA/M6P-GFLG-HPMA-GFLG- ${ }^{32} \mathrm{P}-\mathrm{TFO}(200: 1)$ incubated for $24 \mathrm{~h}$ at $37^{\circ} \mathrm{C}$ with papain. 


\subsubsection{Inhibition of Collagen Type I Gene Expression by M6P-GFLG-HPMA-GFLG-TFO}

To confirm that TFO conjugation to M6P-HPMA does not adversely affect its ability to inhibit the transcription of type I collagen, we transfected HSC-T6 cells with TFO and M6P-GFLG-HPMA-GFLG-TFO. Compared to the control cells treated with M6P-HPMA (lane 1), type I collagen gene expression by the cells treated with M6P-GFLG-HPMA-GFLG-TFO (lane 2) decreased as efficiently as that by the cells treated with the TFO (lane 3) (Figure 3-6). Since the molecular weight of collagen type 1 precursor is $130-140 \mathrm{kDa}$ and that of mature collage type I is $70-90 \mathrm{Da}$, there were 2 bands for collagen.

\subsubsection{Biodistribution of M6P-GFLG-HPMA-GFLG-TFO}

Following synthesis and purification of M6P-GFLG-HPMA-GFLG- ${ }^{32} \mathrm{P}-\mathrm{TFO}$, we determined the biodistribution of this conjugate at 2.5, 5, 15,30, 60 and 90 min post injection into the rat tail vein. Figure 3-7 shows the time course of radioactivity in the plasma, urine, liver, kidney, lung, and heart. Conjugation with M6P-GFLG-HPMA-GFLG -ONP significantly increased the accumulation of ${ }^{32} \mathrm{P}-\mathrm{TFO}$ in the liver, as compared to our previously reported biodistribution data of free ${ }^{32} \mathrm{P}-\mathrm{TFO} .{ }^{38}$ Almost $70 \%$ of the conjugate accumulated in the liver at $30 \mathrm{~min}$ postinjection and $60 \%$ at 60 min postinjection (Figure 3-7), however, only $40 \%$ of free ${ }^{32} \mathrm{P}-\mathrm{TFO}$ was in the liver at $30 \mathrm{~min} .{ }^{38}$ Figure 3-8 shows the tissue concentration of M6P-GFLG-HPMA-GFLG- ${ }^{32} \mathrm{P}-\mathrm{TFO}$ in the plasma $(\mu \mathrm{g} / \mathrm{mL})$ and tissues $(\mu \mathrm{g} / \mathrm{g})$ after tail vein injection into rats. The conjugation with M6P-GFLG-HPMA-GFLG-ONP significantly increased the accumulation of radioactivity in the liver and was the highest at $30 \mathrm{~min}$ post-injection. The conjugate concentration was high not only in the liver, but also in the spleen and kidney. However, radioactivity in the kidney rapidly decreased with time. We also included the tissue accumulation data of ${ }^{32} \mathrm{P}-\mathrm{TFO}$ at $30 \mathrm{~min}$ post-injection for comparison.

We also calculated the pharmacokinetic profiles using two-compartment model by plotting plasma concentration versus time, analyzing data using WinNonlin professional software. As shown in Figure 3-9, there was good fit between observed and predicted results. Plasma elimination of M6P-GFLG-HPMA-GFLG- ${ }^{32} \mathrm{P}-\mathrm{TFO}$ was biphasic with a

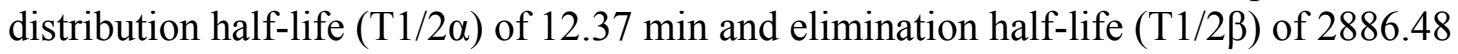
min. Figure 3-9 also summarizes the pharmacokinetic parameters such as AUC, Vd and CL. Consistent with the rapid clearance $(0.059 \mathrm{~mL} / \mathrm{min})$, M6P-GFLG-HPMA-GFLG- ${ }^{32}$ P-TFO had a large Vd of $231.33 \mathrm{~mL}$, Table 3-1 summarizes the AUC, tissue uptake rate index, and organ clearance for representative organs at 90 min after systemic administration of ${ }^{32} \mathrm{P}-\mathrm{TFO}$ and M6P-GFLG-HPMA-GFLG ${ }^{32} \mathrm{P}-\mathrm{TFO}$ in rats. The liver uptake rate indices and clearance of the conjugate were significantly higher than those of ${ }^{32} \mathrm{P}$-TFO: $2176 \pm 58$ versus $376 \pm 38$ $\mu \mathrm{L} / \mathrm{h} / \mathrm{g}$ and $11531 \pm 308$ versus $2218 \pm 206 \mu \mathrm{L} / \mathrm{h}$, respectively. The tissue uptake rate indices and organ clearances of heart, kidney, and lung were significantly higher than 


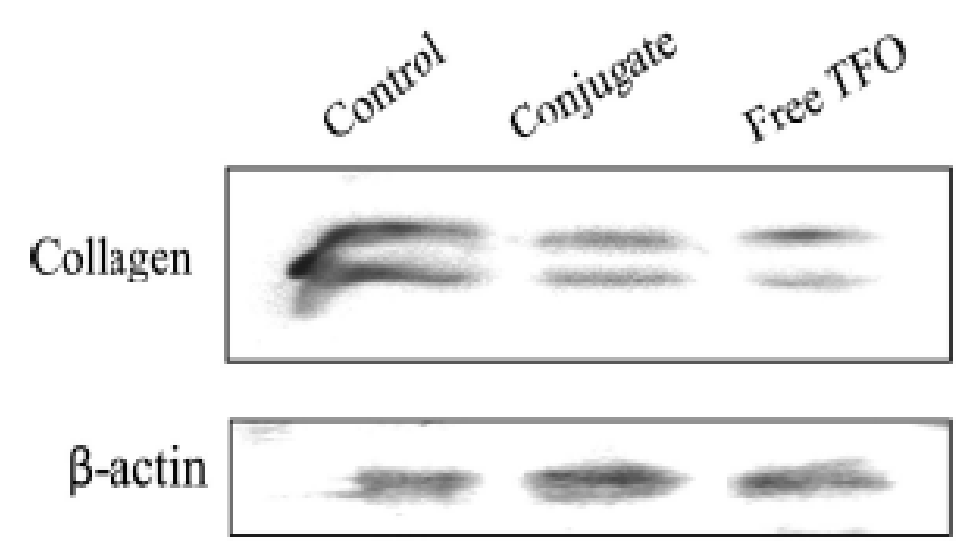

Figure 3-6 Inhibition of collagen gene expression. Transfection of HSC-T6 cells with TFO (lane 3) and M6P-GFLG-HPMA-GFLG-TFO (lane 2) inhibited collagen gene expression compared to the control group (lane 1). 


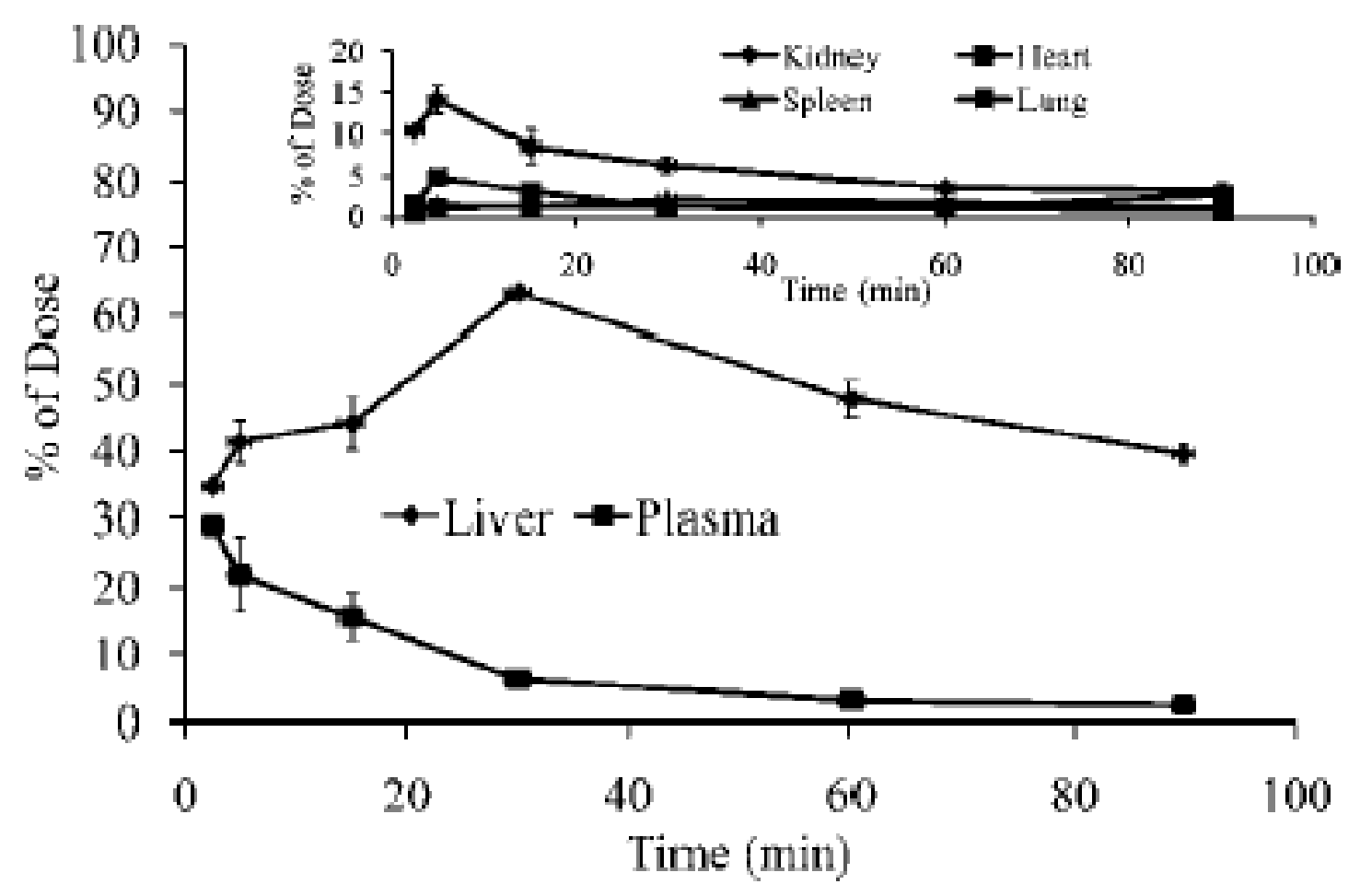

Figure 3-7 Biodistribution of M6P-GFLG-HPMA-GFLG- ${ }^{32} \mathrm{P}-\mathrm{TFO}$ and free ${ }^{32} \mathrm{P}-\mathrm{TFO}$ after tail vein injection into rats at a dose of $0.2 \mathrm{mg} \mathrm{TFO} / \mathrm{kg}$ of body weight. 


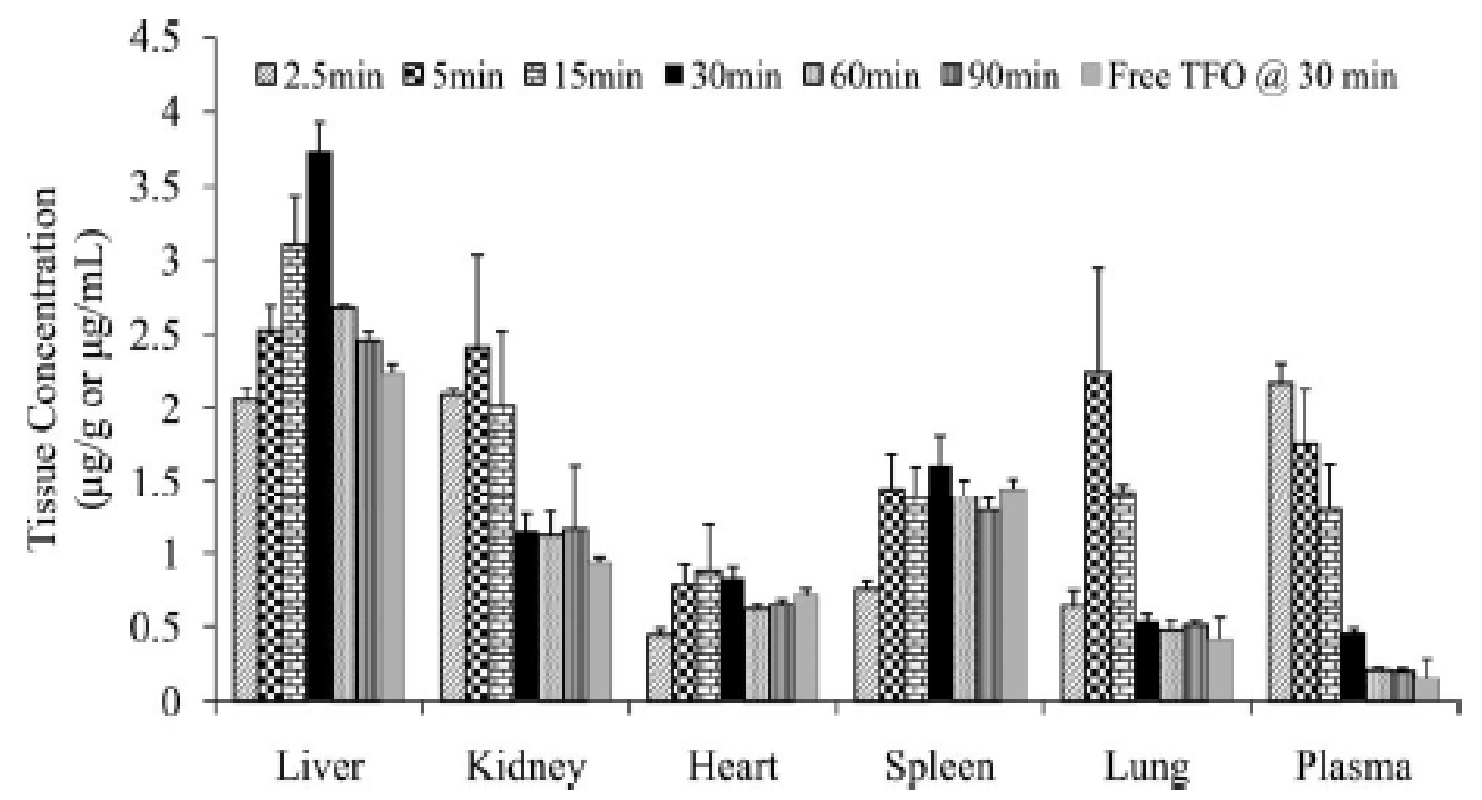

Figure 3-8 Concentration time profiles of radioactivity in different organs and the plasma after tail vein injection of M6P-GFLG-HPMA-GFLG- ${ }^{32} \mathrm{P}-\mathrm{TFO}$ and free ${ }^{32} \mathrm{P}-\mathrm{TFO}$ into rats at a dose of $0.2 \mathrm{mg} \mathrm{TFO} / \mathrm{kg}$ of body weight. 


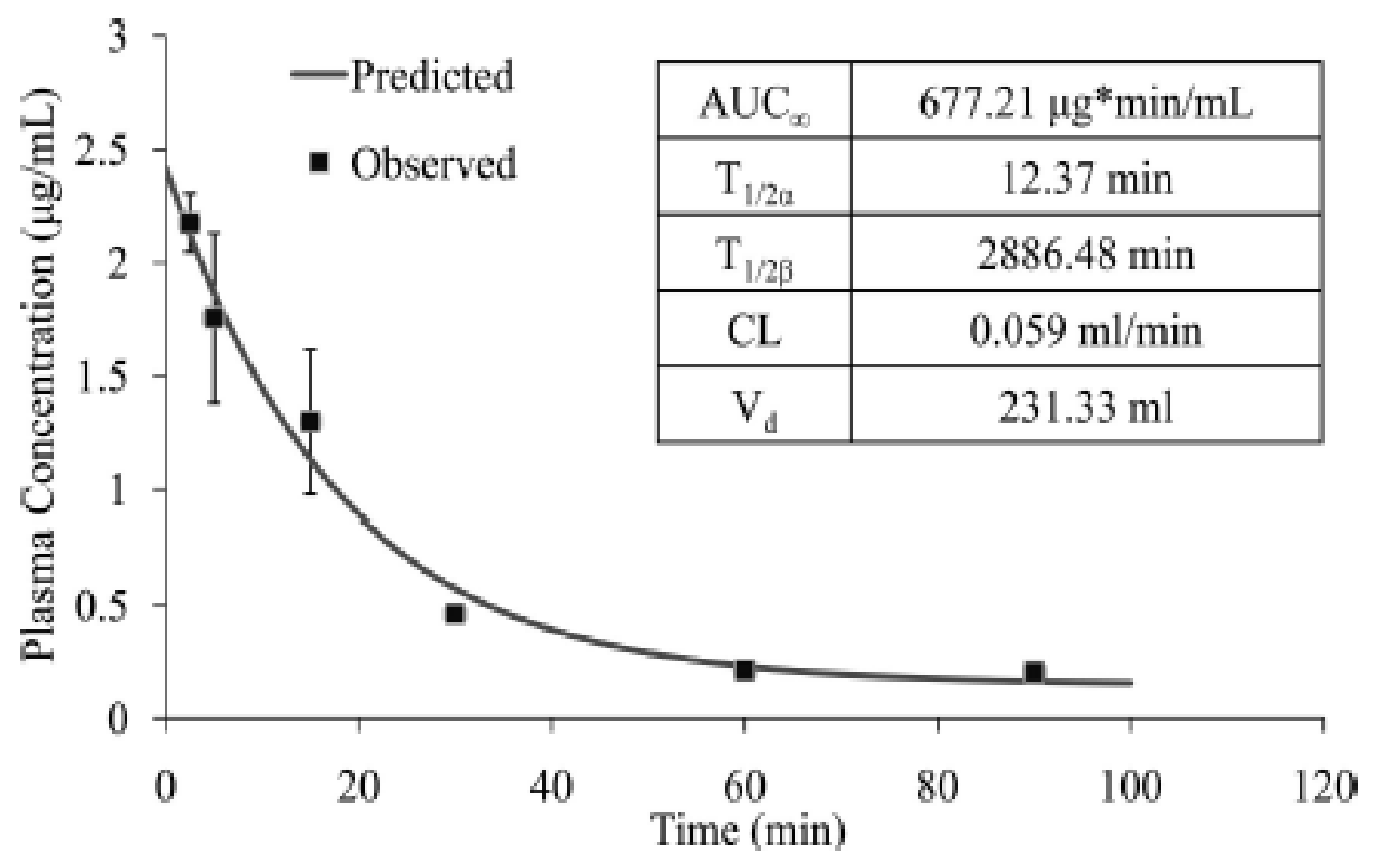

Figure 3-9 Pharmacokinetic profiles of M6P-GFLG-HPMA-GFLG- ${ }^{32} \mathrm{P}-\mathrm{TFO}$ at $30 \mathrm{~min}$ after post vein injection. Plasma data was analyzed using a two-compartment model with WinNonlin Enterprise (v 5.2) software. AUC: area under the curve; CL: clearance; $\mathrm{V}_{\mathrm{d}}$ : volume of distribution. 
Table 3-1 Tissue uptake rate index and clearance of ${ }^{32} \mathrm{P}-\mathrm{TFO}$ and M6P-GFLG-HPMA-GFLG- $-{ }^{32} \mathrm{P}-\mathrm{TFO}$ after systemic administration into rats.

\begin{tabular}{|c|c|c|c|c|c|c|c|c|c|c|c|}
\hline \multirow{2}{*}{ Sample } & \multirow{2}{*}{$\begin{array}{c}\mathrm{AUC}_{90} \\
(\mu \mathrm{g} / \mathrm{min} \\
/ \mathrm{mL})\end{array}$} & \multicolumn{5}{|c|}{ Tissue uptake rate index $(\mu \mathrm{L} / \mathrm{h} / \mathrm{g})$} & \multicolumn{5}{|c|}{ Organ clearance $(\mu \mathrm{L} / \mathrm{h})$} \\
\hline & & Liver & Kidney & Heart & Spleen & Lung & Liver & Kidney & Heart & Spleen & Lung \\
\hline Conjugate & 67.11 & $2176 \pm 58$ & $1033 \pm 391$ & $578 \pm 31$ & $1146 \pm 68$ & $450 \pm 112$ & $11531 \pm 308$ & $1137 \pm 430$ & $318 \pm 17$ & $436 \pm 26$ & $383 \pm 26$ \\
\hline TFO & 95.15 & $376 \pm 38$ & $235 \pm 29$ & $49 \pm 3$ & $205 \pm 10$ & $55 \pm 9$ & $2218 \pm 206$ & $241 \pm 41$ & $23 \pm 2$ & $83 \pm 7$ & $42 \pm 8$ \\
\hline
\end{tabular}


those of ${ }^{32} \mathrm{P}-\mathrm{TFO}$, possibly due to increase in vivo stability of TFO upon conjugation with M6P-GFLG-HPMA-GFLG-ONP.

\subsubsection{Effect of Fibrosis on Biodistribution}

We recently reported significant decrease in the hepatic uptake of ${ }^{33} \mathrm{P}-\mathrm{TFO}$ when injected into fibrotic rats. ${ }^{38}$ To make sure that there is no decrease in the hepatic uptake of M6P-GFLG-HPMA-GFLG- ${ }^{32}$ P-TFO, we determined the bio-distribution of this conjugate at 30 min post injection in fibrotic rats. To exclude the possibility that HPMA, the backbone, is not involved in the specific uptake of the TFO, we also determined the biodistribution of HPMA-GFLG- ${ }^{32} \mathrm{P}-\mathrm{TFO}$ at $30 \mathrm{~min}$ post-injection. There was no decrease in the hepatic uptake of M6P-GFLG-HPMA-GFLG- ${ }^{32} \mathrm{P}-\mathrm{TFO}$ in fibrotic rats (Figure 3-10). As expected, HPMA-GFLG- ${ }^{32} \mathrm{P}-\mathrm{TFO}$ accumulation in the liver was much less than that of M6P-GFLG-HPMA-GFLG- ${ }^{32} \mathrm{P}-\mathrm{TFO}$ and decreased when fibrotic rats were used (Figure 3-10). This may be due to the fact that M6P/IGFII receptors are up-regulated during fibrosis, ${ }^{214}$ which should increase the uptake by HSCs via receptor mediated endocytosis and cancel out any adverse effect created by decrease in sinusoidal gap.

\subsubsection{Competition in Hepatic Uptake}

To determine whether the hepatic uptake of M6P-GFLG-HPMA-GFLG- ${ }^{32} \mathrm{P}-\mathrm{TFO}$ is mediated by M6P/IGFII receptor mediated endocytosis, we preinjected excess amount of

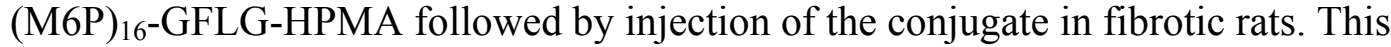
resulted in a significant decrease in the hepatic uptake of the conjugate in fibrotic rats (Figure 3-11), possibly due to M6P/IGFII receptor mediated endocytosis of the conjugates.

\subsubsection{Hepatic Cellular Localization}

To determine the uptake of M6P-GFLG-HPMA-GFLG- ${ }^{32} \mathrm{P}-\mathrm{TFO}$ by different liver cell types, the livers of normal and fibrotic rats were perfused at $30 \mathrm{~min}$ post tail vein injection, and hepatocytes, HSCs, Kupffers and endothelial cells were isolated for determining the amount of radioactivity in these cells. As shown in Figure 3-12, HSCs were the major site for the uptake of this conjugate. Furthermore, the uptake of M6P-GFLG-HPMA-GFLG- ${ }^{32}$ P-TFO by HSCs was significantly higher compared to free ${ }^{32} \mathrm{P}-\mathrm{TFO}$. Although there was no significant decrease in the overall hepatic recovery of M6P-GFLG-HPMA-GFLG- ${ }^{32} \mathrm{P}-\mathrm{TFO}$, uptake by the HSCs of DMN-induced liver fibrotic rats was much higher than that of normal rats $(79.63 \pm 2.56 \%$ versus $51.55 \pm 2.02 \%)$ (Figure 3-12). We also perfused the livers of both normal and fibrotic rats at $30 \mathrm{~min}$ post-injection of HPMA-GFLG- ${ }^{32} \mathrm{P}-\mathrm{TFO}$ and determined the intrahepatic distribution of the TFO by different liver cells. As can be seen in Figure 3-12, the conjugation of

${ }^{32} \mathrm{P}-\mathrm{TFO}$ with GFLG-HPMA did not significantly increase the TFO uptake by HSCs of 


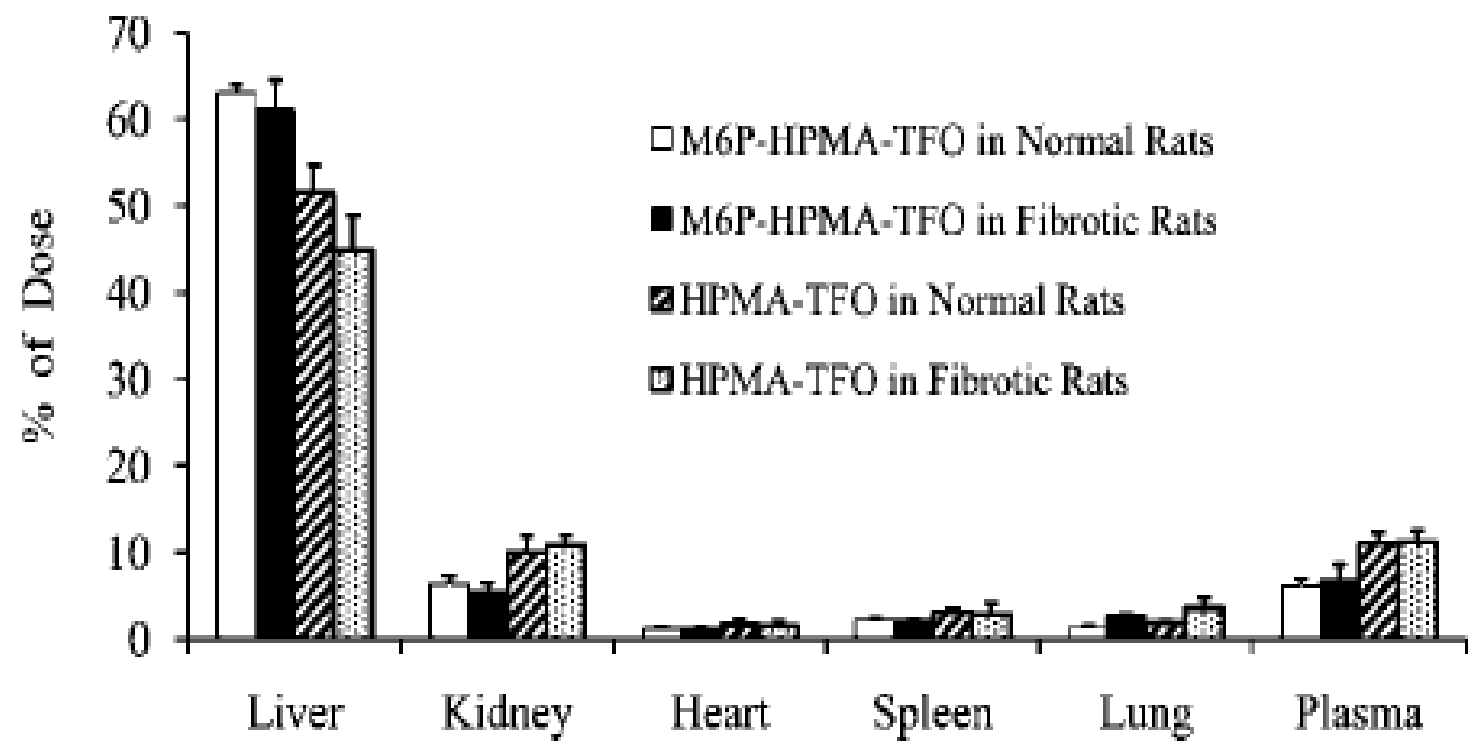

Figure 3-10 Effect of fibrosis on hepatic uptake of M6P-GFLG-HPMA-GFLG- ${ }^{32} \mathrm{P}-\mathrm{TFO}$ and HPMA- ${ }^{32} \mathrm{P}-\mathrm{TFO}$ after systemic administration into DMN-induced fibrotic rats. At 30 min post-injection of this conjugate $\left(1 \times 10^{6} \mathrm{cpm}\right)$ at a dose of $0.2 \mathrm{mg} / \mathrm{kg}$, blood was collected by cardiac puncture. Rats were sacrificed; major organs were isolated, washed with saline, and subjected into scintillation counting. Values are the mean \pm SD of 3 rats. 


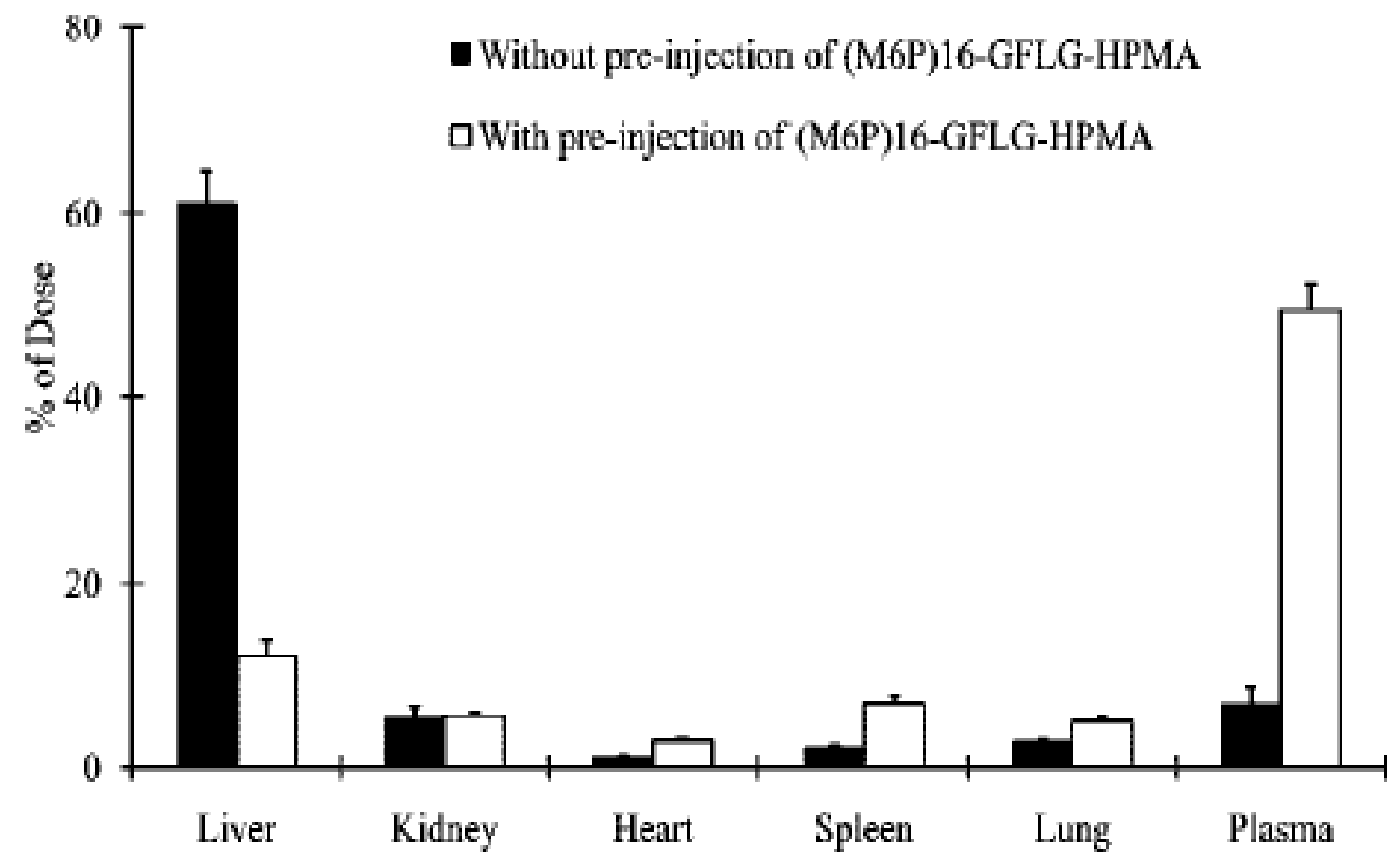

Figure 3-11 Effect of excess M6P-GFLG-HPMA on the biodistribution of M6P-GFLG-HPMA-GFLG- ${ }^{32} \mathrm{P}-\mathrm{TFO}$ in fibrotic rats. 
(A) Normal Rats

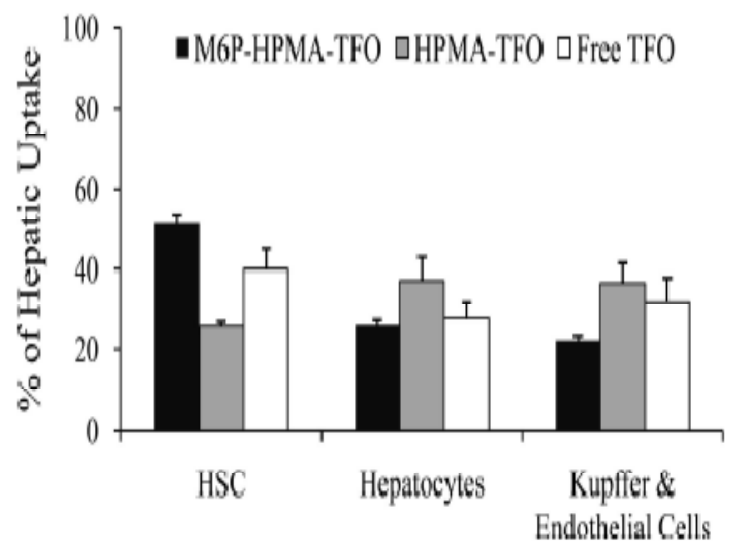

(B) Fibrotic Rats

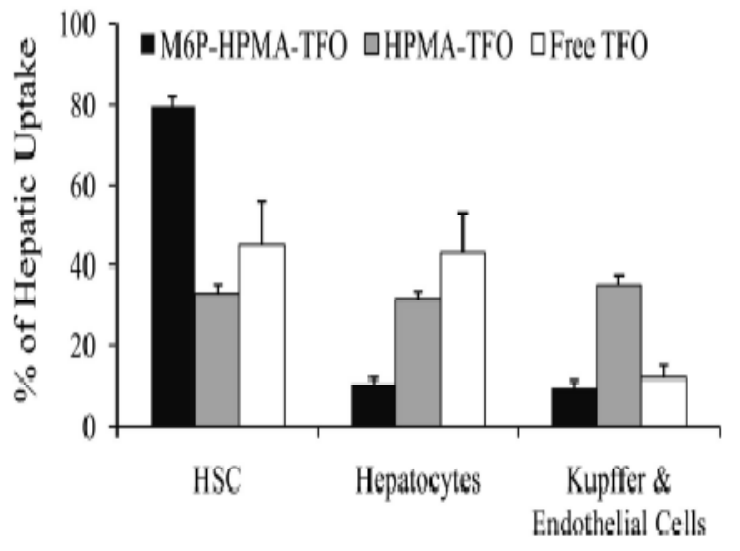

Figure 3-12 Intrahepatic distribution of M6P-GFLG-HPMA-GFLG- ${ }^{32} \mathrm{P}-\mathrm{TFO}$ in normal and fibrotic rats. The liver was perfused in situ by collagenase/pronase digestion at 30 min post-injection of M6P-GFLG-HPMA-GFLG- ${ }^{32} \mathrm{P}-\mathrm{TFO}$, HPMA-GFLG- ${ }^{32} \mathrm{P}-\mathrm{TFO}$, or ${ }^{32} \mathrm{P}-\mathrm{TFO}$ at dose of $0.2 \mathrm{mg}$ TFO $/ \mathrm{kg}$ of body weight. Hepatocytes, Kupffer and endothelial cells, and hepatic stellate cells (HSC) were separated, and the associated radioactivity was measured. The contribution of each liver cell type was exposed as percentage of total liver uptake. Results are expressed as the mean $\pm \mathrm{SD}(\mathrm{n}=3)$. 
both normal and fibrotic rats, suggesting the HPMA backbone is not involved in the specific uptake of the TFO.

\subsection{Discussion}

Excessive production of extracellular matrix (ECM), primarily type I collagen, by activated HSCs is known to be one of the major causes of liver fibrosis. ${ }^{197}$ The entire region spanning from -140 to -200 of $\alpha 1$ (I) collagen gene promoter exist as a symmetric polypurine-polypyrimidine tract in which the polypyrimidine sequence at -141 to -170 is called $\mathrm{C} 1$ region present on the non-coding strand, whereas the adjacent polypurine sequence from -171 to -200 is called $\mathrm{C} 2$ region located on the coding strand. The cis-acting element is the $\mathrm{C} 1$ and $\mathrm{C} 2$ regions, playing an important role in collagen transcription. ${ }^{35}$ We have previously demonstrated that TFOs could form triplex in this region and inhibit the activity of $\alpha 1$ (I) collagen promoter in vitro. ${ }^{34,36}$ In addition, TFOs have been shown to partly reverse fibrosis in a dimethylnitrosamine (DMN) induced liver fibrosis rats, indicating the therapeutic potential of these TFOs. ${ }^{38}$

M6P is a ligand for M6P/IGF II receptor, which is up-regulated in activated HSCs during liver fibrosis, enabling selective accumulation of M6P-conjugated carrier or drug molecules. ${ }^{215}$ M6P conjugation to albumin increased the uptake of M6P-BSA by HSCs in liver fibrotic rats. ${ }^{216}$ Conjugation of M6P to virus recombinant interleukein-10 (vIL-10) significantly increased the hepatic uptake of $v$ IL-10 by the liver.

To avoid the use of polycations, Rajur et al. conjugated ODNs to asialoorosomucoid via disulfide bond. ${ }^{217}$ However, this strategy is not suitable for the TFO, since direct conjugation of molecules to the TFOs often disrupts the triplex-forming ability of the TFOs, which is essential for transcription inhibition. Furthermore, liver fibrosis results in the loss of sinusoidal fenestrae, suggesting that particulate delivery systems may not be good for TFO delivery to the HSCs.

To avoid any non-specific ionic interaction and the large particle size, we recently conjugated TFO to M6P-BSA via a disulfide bond and demonstrated enhanced uptake by the HSCs after systemic administration of M6P-BSA-TFO into normal and fibrotic rats. ${ }^{218}$ However, repeated administration of this conjugate is likely to cause immune reaction due to BSA and thus we synthesized M6P-GFLG-HPMA-GFLG-ONP and conjugated to TFO, where GFLG is a lysosomally degradable tetrapeptide, and thus will facilitate lysosomal release of TFO and its escape to the cytoplasm after cellular uptake. Following synthesis and characterization using RP-HPLC and GPC, we determined whether TFO could be dissociated from the conjugate by incubating M6P-GFLG-HPMA-GFLG-TFO with papain and TFO release was monitored. As shown in Figure 3-4, free TFO release concentration increased with incubation time with papain, which is consistent with the literature. ${ }^{213}$ Papain is a cysteine protease hydrolase enzyme and belongs to the same family as cathepsin B, which is the most important enzyme in the lysosomes to cleave GFLG spacer. ${ }^{210,212,213}$ 
To make sure the TFO released from the conjugate can form a triplex to the target duplex DNA, we incubated M6P-GFLG-HPMA-GFLG-TFO with papain for $24 \mathrm{~h}$ and then used the released TFO to form triplex with the target duplex DNA. As can be seen in Figure 3-5, the TFO released from the conjugate formed triplex with duplex DNA.

To confirm that TFO conjugation to M6P-GFLG-HPMA does not adversely affect its ability to inhibit the transcription of type I collagen, we transfected HSC-T6 cells with M6P-GFLG-HPMA-GFLG-TFO. Compared to the control cells treated with M6P-HPMA, type I collagen gene expression by the cells treated with M6P-GFLG-HPMA-GFLG-TFO decreased as efficiently as that by the cells treated with the TFO (Figure 3-6).

Conjugation of TFO to M6P-GFLG-HPMA-GFLG-ONP significantly increased TFO delivery to the liver from $47 \%$ for ${ }^{32} \mathrm{P}-\mathrm{TFO}$ to $70 \%$ for M6P-GFLG-HPMA-GFLG- ${ }^{32} \mathrm{P}-\mathrm{TFO}$ at $30 \mathrm{~min}$ post injection. To determine whether the hepatic uptake of M6P-GFLG-HPMA-GFLG- ${ }^{32} \mathrm{P}-\mathrm{TFO}$ is mediated by M6P/IGF II receptor mediated endocytosis, we preinjected rats with excess amount of M6P-GFLG-HPMA-GFLG-ONP. This resulted in a significantly decrease in the hepatic accumulation of this conjugate in fibrotic rats (Figure 3-11). This confirmed the receptor-mediated uptake of this conjugate, since M6P/TGF II receptor is up-regulated in liver fibrotic rats, the hepatic accumulation was almost completely inhibiting by saturating this receptor. Rachmawati et al also demonstrated almost 50\% inhibition in the hepatic uptake of M6P-IL-10 conjugates in fibrotic rats. ${ }^{219}$

To determine the hepatic cellular localization, the liver was perfused at $30 \mathrm{~min}$ post-injection of M6P-GFLG-HPMA-GFLG- ${ }^{32} \mathrm{P}-\mathrm{TFO}$, HPMA-GFLG- ${ }^{32} \mathrm{P}-\mathrm{TFO}$ or ${ }^{32} \mathrm{P}-\mathrm{TFO}$ and different liver cells were separated by fractionation on the Histodenz gradient. As shown in Figure 3-12, almost $80 \%$ of the total liver uptake was contributed by HSCs cells of fibrotic rats, but only $55 \%$ by HSCs of normal rats. The percentage of uptake by hepatocytes was relatively low in both normal and fibrotic rats. However, the conjugation of TFO with GFLG-HPMA did not significantly increase the TFO uptake by HSCs of both normal and fibrotic rats (Figure 3-12); suggesting the HPMA backbone is not involved in the specific uptake of the TFO.

In conclusion, conjugate with M6P-GFLG-HPMA-GFLG-ONP significantly enhance the uptake of the TFO by HSCs and thus this conjugate may be suitable for inhibiting $\alpha 1$ (I) collagen gene expression by HSCs and potentially treat liver fibrosis. 


\section{CHAPTER 4. TREATMENT OF LIVER FIBROSIS AFTER SYSTEMIC ADMINISTRATION OF M6P-HPMA-TFO}

\subsection{Introduction}

Liver fibrosis is the excessive accumulation of extracellular matrix (ECM) proteins including type I collagen in the liver due to chronic hepatitis $\mathrm{C}(\mathrm{HCV})$, alcohol abuse and cholestasis. ${ }^{20}$ There is no drug available to really treat liver fibrosis until now. The commonly used method to treat liver fibrosis is to control the liver inflammation, ${ }^{221}$ as the inflammatory cytokines released due to liver injury activate hepatic stellate cells (HSCs), which are known to be vitamin A and fat storing cells. Following activation, HSCs transdifferentiate into myofibroblast-like cells and acquire contractile and profibrogenic properties, resulting in increased synthesis of type I collagen and other ECM proteins. ${ }^{201}$ Therefore, the reduction of excessive production of ECM, especially type I collagen is crucial to reverse hepatic fibrosis.

Type I collagen is the major component of ECM in the fibrotic liver. It is a heterotrimer composd of two $\alpha 1$ chains and one $\alpha 2$ chain. These chains are encoded by two distinct genes, COL1A1 and COL1A2, respectively. ${ }^{35}$ Recently, we used an antiparallel polypurine phosphorothioate TFO, which can form a triplex with the $\mathrm{C} 1$ sequence located in the rat $\alpha 1$ (I) collagen promoter, ${ }^{34}$ resulting in the transcription inhibition of type $\alpha 1$ (I) collagen. Systemic administration of TFO could decrease liver injury, inflammation and fibrosis. Common bile duct ligation (CBDL) leads to the formation of $\alpha$-smooth muscle action (SMA) positive myofibroblasts, which produce excessive type I collagen and fibrogenic cytokines and resulted in excessive accumulation of ECM.

TFOs distribute throughout the body after systemic administration, with higher accumulation in the liver and kidney. ${ }^{38}$ However, the cellular population which plays a major role in producing collagen in livers is HSCs. Therefore, it is critical to target TFOs to HSCs for efficient anti-fibrotic therapy. M6P/IGF II receptors on the surface of HSCs are up-regulated in fibrotic livers. We have demonstrated that TFO conjugated to mannose-6-phosphate bovine serum albumin (M6P-BSA) enhances TFO accumulation to HSCs. ${ }^{41}$ Since the repeated injection of M6P-BSA-TFO may not be good due to the potential immune reaction of high molecular weight globular BSA (66430 Da) and the closure of sinusoid gap in fibrotic livers, an alternative carrier with less immune reaction, lower molecular weight and size becomes necessary. Therefore, we conjugated M6P via GFLG linker to N-(2-hydroxypropyl) methacrylamide (HPMA) copolymer and then to TFO for target delivery to activated HSCs in the fibrotic liver. ${ }^{27}$ This conjugate enhanced both the elimination half life and target delivery of TFO.

In this chapter, we determined the effect of M6P-HPMA-TFO on treating liver fibrosis in rats undergoing CBDL. Following the systemic administration of M6P-HPMA-TFO three times a week for two weeks, liver samples were analyzed by real 
time RT-PCR, Western blot, histochemistry and immunofluorescence staining to determine the extent of liver fibrosis.

\subsection{Methods and Materials}

\subsubsection{Materials}

Poly (HPMA-co-GFLG-ONP) was purchased from Varian Inc. (Amherst, MA). p-Nitrophenyl- $\alpha$-D-mannopyranoside (pnpM), phosphorus oxide chloride, palladium (10 wt $\%$ on activated carbon), N,N'-diisopropylethylamine, hydroxyproline, Chloramine-T, 3,3 -diaminobenzidine (DAB) and goat serum were purchased from Sigma-Aldrich (St. Louis, MO). Dialysis tubing (molecular weight cutoff $1000 \mathrm{Da}$ ) was purchased from Spectrum Laboratories, Inc. (Houston, TX). Sephadex G75 (superfine) was procured from Pharmacia Fine Chemicals (Uppsala, Sweden). Rabbit anti-rat and goat anti-rat transforming growth factor (TGF)- $\beta 1$ and $\beta$-actin primary antibodies, bovine anti-mouse, donkey anti-goat and donkey anti-rabbit secondary antibodies were purchased from Santa Cruz Biotech, Inc. (Santa Cruz, CA). Other primary and secondary antibodies are all from Abcam Inc. (Cambridge, MA). Tumor necrosis factor (TNF)- $\alpha$ enzyme-linked immunosorbent assay (ELISA) kit was purchased from eBioscience, Inc. (San Diego, CA). Serum alanine transaminase (ALT) and aspartate transaminase (AST) kits were purchased from ID Labs, Inc (London, Canada). Citric acid and sodium citrate were procured from Curtin Matheson Scientific, Inc (Houston, TX). Sodium hydroxide was purchased from Fisher Scientific (Fair Lawn, NJ). TFO, which was a 25 mer anti-parallel fully phosphorothioate ODN (3'-GAGGGGGGAGGAGGGAAAGGAAGGG-5') targeting rat $\alpha 1$ (I) collagen gene promoter, and TFO-3'- $\mathrm{NH}_{2}$ were synthesized by Invitrogen (Carlsbad, CA). Isoflurane was purchased from Baxter Pharmaceutical Products, Inc. (Deerfield, IL). All solvents and chemicals used in this study were used as available without further purification.

\subsubsection{Synthesis and In Vitro Characterization of M6P-HPMA-TFO Conjugate}

For targeted delivery, TFO was conjugated to M6P-GFLG-HPMA as described previously. ${ }^{27}$ The conjugate was dialyzed against $\mathrm{H}_{2} \mathrm{O}$ for $24 \mathrm{~h}$, purified on a Sephadex G75 Gel column with pure water, freeze-dried, kept at $-80^{\circ} \mathrm{C}$, and resuspended in steriled saline before use. ${ }^{27}$ The conjugation was confirmed by polyacrylamide gel electrophoresis as described before. We also confirmed this by ${ }^{1} \mathrm{H}$ nuclear magnetic resonance (NMR) (JOEL, $\left.270 \mathrm{MHz}, \mathrm{T}=25^{\circ} \mathrm{C}\right)$ using deuterated water $\left(\mathrm{D}_{2} \mathrm{O}\right)$ as solvent. The chemical shifts were calibrated using $\mathrm{D}_{2} \mathrm{O}$ as an internal reference and given in parts per million.

TFO and M6P-HPMA-TFO solution dissolved in saline were injected to rats every two days by tail veins at the same dose of $8 \mathrm{mg} / \mathrm{kg}$. 


\subsubsection{Animals}

Male Sprague Dawley rats weighing 200-250 g were purchased from Harlan Co. (San Diego, CA) and were housed individually under the conditions as per the NIH (http://grants1.nih.gov/grants/olaw/references/phspol.html) and Institutional Animal Care and Use committee (IACUC) using the approved protocols. All animals were housed in microisolator cages in virus-free facilities and fed laboratory chow and water ad labium.

\subsubsection{Bile Duct Ligation and Treatment}

Common bile duct ligation (CBDL) or sham operation was performed. In the first day of CBDL, animals were divided into 4 groups: sham (midline abdominal incision and closure), CBDL, CBDL+TFO; and CBDL+M6P-HPMA-TFO. TFO and its conjugate were given via the tail vein injection from the first day of CBDL three times a week for two consecutive weeks. At the end of experiment, blood was collected when animals were sacritificed under isoflurane anesthesia by puncture of the right heart vehicle and exsanguinations. Liver tissues were collected, washed, blotted dry and frozen.

\subsection{5. $\quad$ Standard Serum Parameters}

Serum levels of aspartate aminotransferase (AST) and alanine aminotransferase (ALT) were used as markers of liver injury. Serum ALT and AST concentrations were measured using IDToxTM Alanine Transaminase color endpoint assay kit and IDToxTM Aspartate Transaminase Enzyme Assay Kit (ID LabsTM Inc, London, ON, Canada) according to the manufacturer's instructions and absorbance was measured using a spectrophotometer. Total bilirubin levels were measured using a MaxDiscovery ${ }^{\mathrm{TM}}$ Total bilirubin assay kit from Bioo scientific (Austin, TX) and absorbance at $560 \mathrm{~nm}$ was measured using a UV spectrophotometer.

\subsubsection{Hydroxyproline Assay}

Hepatic hydroxyproline content was quantified colorimetrically from $30 \mathrm{mg}$ of fresh liver tissue as previously described after hydrolyzing with $6 \mathrm{~N} \mathrm{HCl}$ and heating in an oven at $110^{\circ} \mathrm{C}$ for $18 \mathrm{~h}$. Citrate buffer and Chloramine-T reagent were added to the samples and allowed to react for $20 \mathrm{~min}$ at room temperature. Fresh prepared Ehrlich's reagent was then added to each sample and allowed to react for 15 min at a warm water bath $\left(60.8^{\circ} \mathrm{C}\right)$. Following cooling to room temperature, absorbance was read at $550 \mathrm{~nm}$ and the results were expressed as micrograms of hydroxyproline per grams of liver. 


\subsubsection{ELISA for Tumor Necrosis Factor- $\alpha$}

TNF- $\alpha$ concentration in serum of different groups were measured using enzyme-linked immunosorbent assay (ELISA), according to the manufacturer's protocol (eBioscience, San Diego, CA).

\subsubsection{Real Time RT-PCR}

Total liver RNA was isolated from $20 \mathrm{mg}$ of liver tissue by RNAeasy Mini Kit (Qiagen, Valencia, CA) and the RNA concentration was measured using a Nanodrop UV spectrophotometer (Thermo Scientific, Pittsburgh, PA). Then, 385 ng of the total RNA per sample was reverse transcribed into cDNA and amplified by real time PCR using SYBR Green-1 dye universal master mix on ABI Prism 7700 Sequence Detection System (Applied Biosystems, Inc., Foster City, CA). The sequences of primers for detecting TGF- $\beta 1, \alpha-$ SMA, TIMP- 1 and $18 \mathrm{~S}$ as an internal control are the same as our previous report. $^{222}$

\subsubsection{Histological Staining}

Formalin-fixed liver specimens from M6P-HPMA-TFO treated, TFO treated, BDL and non-fibrotic rats were dehydrated in $70 \%$ alcohol, incubated in xylene, and embedded in paraffin. Five-micron-thick tissue sections were cut and stained with Masson's Trichrome staining for collagen.

\subsubsection{Immunofluorescent Staining}

Immunofluorescent staining was performed on snap frozen liver tissue as described before. Briefly, $14 \mu \mathrm{m}$ cryosections were cut on lysine coated slides and fixed in $95 \%$ cold ethanol. Slides were air dried and stored at $-80^{\circ} \mathrm{C}$ till further use. The sections were blocked with $10 \%$ goat serum with $1 \%$ BSA in PBS for $1 \mathrm{~h}$ at room temperature and then incubated with the following primary antibodies, rabbit anti-rat $\alpha$-SMA, mouse anti-rat cytoketain-7 and mouse anti-rat CD11 for $1 \mathrm{~h}$ at room temperature. Then anti-rabbit Alexa Fluor 488 and anti-mouse Alexa Fluor 594 were applied to the cytosections. Nuclear staining was performed using 4',6-diamidino-2-phenylindole (DAPI) and was visualized on a Zeiss Apoplan Microscope.

\subsubsection{Western Blot}

Effect of M6P-HPMA-TFO conjugates on TGF- $\beta 1, \alpha-S M A$ and CK-7 was determined by Western blot analysis as described. ${ }^{222}$ Briefly, the liver tissues were homogenized and then lysed using Laemmli sodium dodecyl sulfate (SDS) buffer. The lysate was then boiled for $10 \mathrm{~min}$ and subjected to $4-15 \%$ precast SDS-polyacrylamide 
gel electrophoresis (SDS-PAGE) and subsequent transfer to Immobilon polyvinylidene fluoride membrane by iBLOT (Invitrogen, Carlsbad, CA). After blocking with 3\% nonfat dry milk in TBST containing $0.05 \%$ Tween- 20 for $1 \mathrm{~h}$ at room temperature, the membranes were incubated with various primary antibodies for $16 \mathrm{~h}$ at $4^{\circ} \mathrm{C}$. $\beta$-Actin polyclonal antibody was used as an internal control. After additional washing in blocking medium, horseradish peroxidase (HRP)-conjugated secondary antibody was added, followed by washing in TBST buffer before visualization using enhanced chemiluminescence (ECL) reaction and X-ray film exposure.

\subsubsection{Statistics Analysis}

Data are expressed as the mean \pm standard deviation (S.D.). Comparison was performed by the one-way ANOVA. $\mathrm{p}<0.05$ was considered statistically significant.

\subsection{Results}

\subsubsection{In Vitro Characterization of M6P-HPMA-TFO}

Following synthesis and purification, M6P-HPMA-TFO was characterized by $1 \mathrm{H}$ NMR. As depicted in Figure 4-1, the chemical shifts at $\delta=7.8$ is characteristics of the $\mathrm{NH}_{2}$ groupsin TFO. $\delta=8.1$ and $\delta=7.6$ are characteristics of ONP groups in HPMA. The chemical shift of protons in TFO base pairs $(\delta=1.2)$ and protons in phenol groups in M6P $(\delta=6.8)$ were found in M6P-HPMA-TFO (arrow heads, Figure 4-1), confirming TFO conjugation to M6P-HPMA-ONP.

\subsubsection{M6P-HPMA-TFO Inhibits Collagen and Attenuates Liver Fibrosis}

To confirm TFO conjugation to M6P-HPMA does not adversely affect the transcription inhibiting ability of TFO, the level of type I collagen gene expression was determined by Western blot analysis. Both TFO and M6P-HPMA-TFO treated groups significantly down-regulated type I collagen expression (Figure 4-2A), with relatively higher level of collagen gene silencing in M6P-HPMA-TFO treated group (Figure 4-2A).

To examine the effect of this conjugate on liver fibrosis, CBDL rat livers were isolated to confirm liver fibrosis by hydroxyproline assay and Masson's trichrome staining for total collagen. There was significant increase in hydroxyproline content in the liver after CBDL, suggesting the liver fibrosis model was successfully established. Hydroxyproline content of the livers of normal, fibrotic, TFO treated and M6P-HPMA-TFO treated rats were $4.64 \pm 1.55,12.56 \pm 1.66,8.51 \pm 1.58$ and $7.04 \pm 1.95 \mu \mathrm{g}$ per mg liver tissues, respectively (Figure 4-2B). Both TFO and M6P-HPMA-TFO injected groups showed significant reduction in total collagen. Compared to TFO treated 


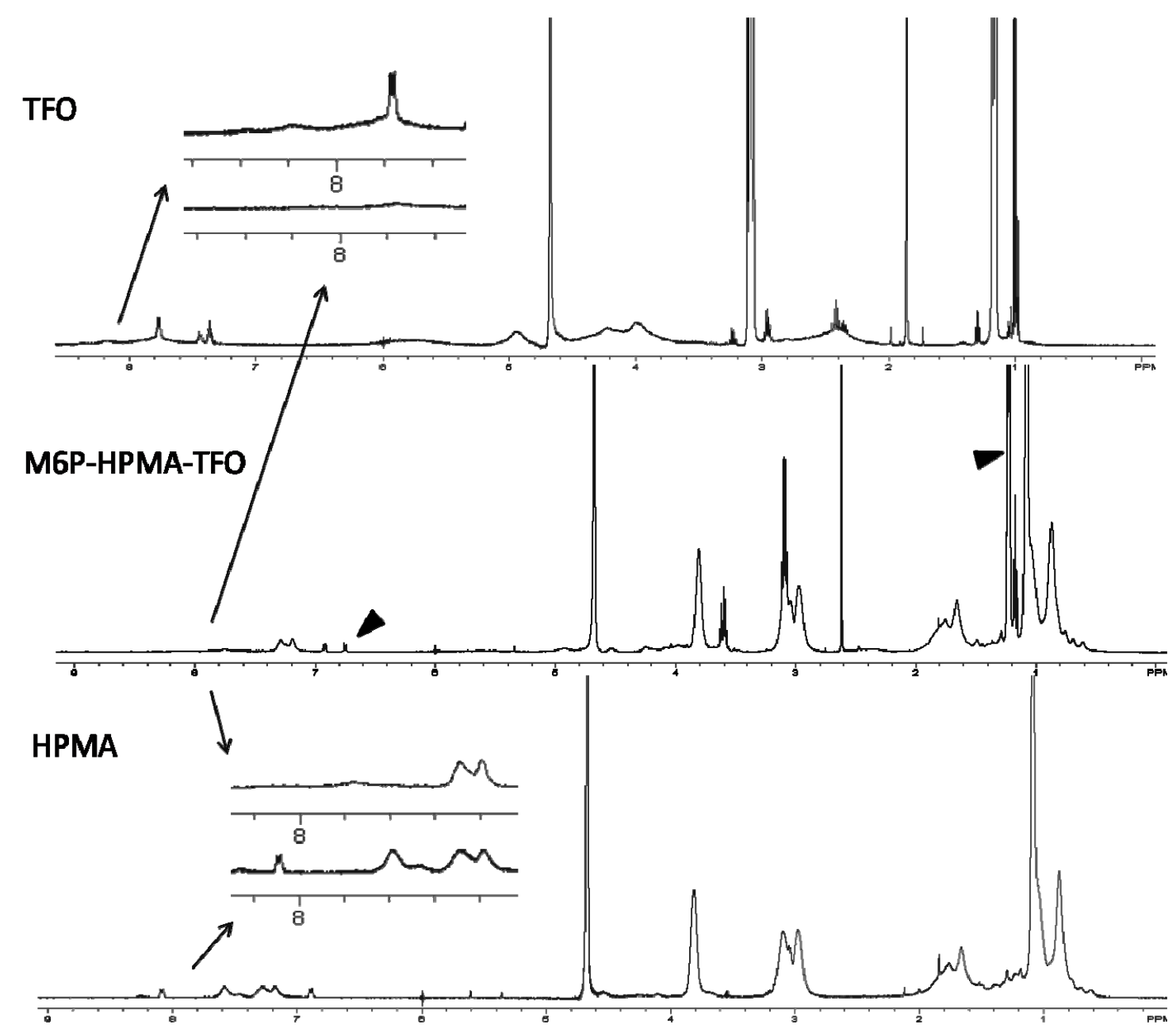

Figure 4-1 NMR characterization of M6P-HPMA-TFO. 


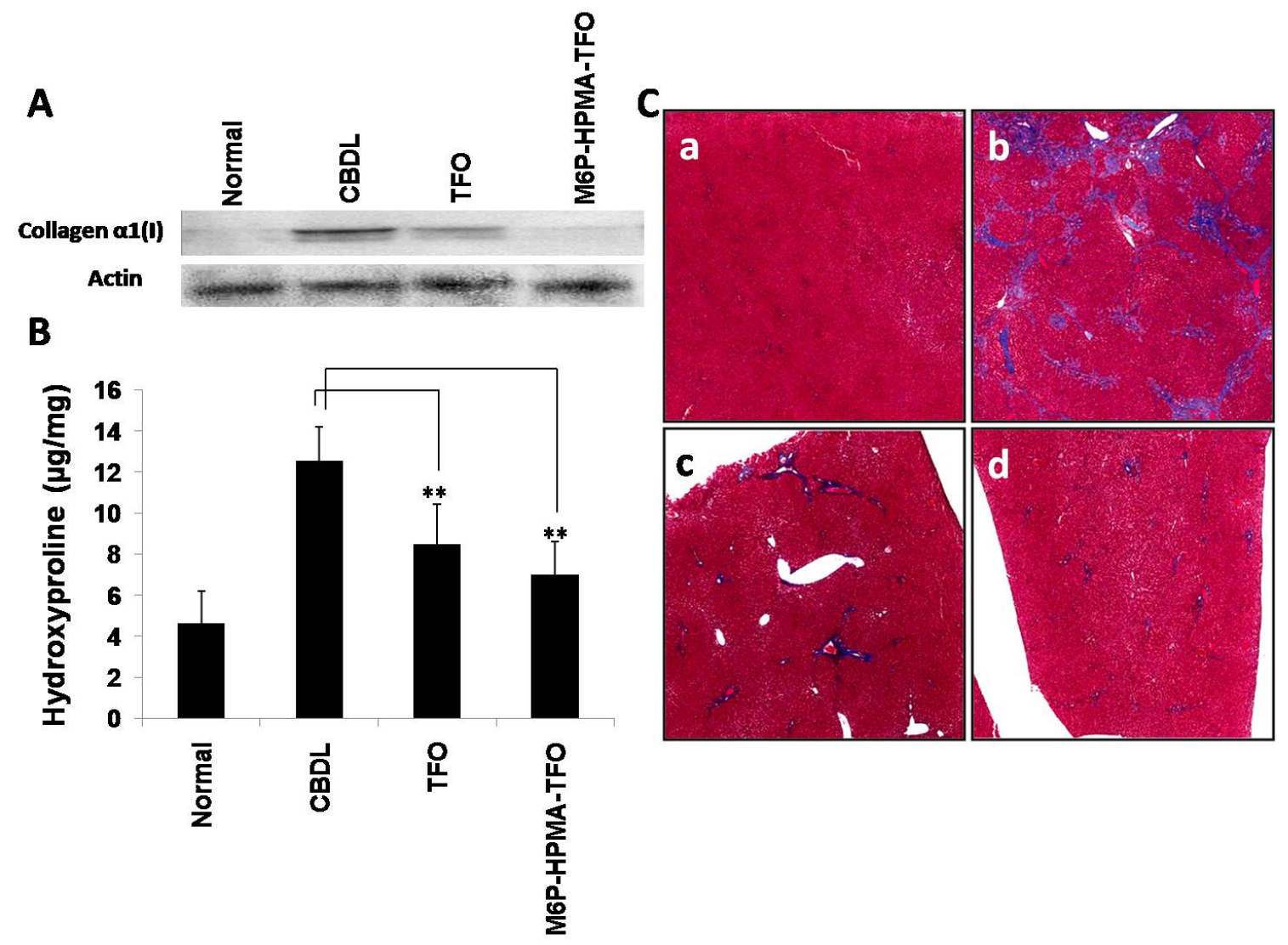

Figure 4-2 Measurement of type I collagen and total collagen. A) Western blot for type I collagen. B) Hydroxyproline level of total collagen. C) Histochemistry staining of total collagen, a) non-fibrotic rat, b) CBDL rat, c) free TFO group, d) M6P-GFLG-HPMA-GFLG-TFO group. 
rats, M6P- HPMA-TFO injected group showed relatively more inhibition of hydroxyproline (Figure 4-2B).

Figure 4-2C shows micrographs of Masson's trichrome staining of rat liver tissues at 2 weeks after CBDL. In sham operated rats, collagen expression was very low (Figure 4-2C.a). In rats administrated with M6P-HPMA-TFO (Fig 4-2C.d) and free TFO (Figure 4-2C.c), there was a significant decrease in collagen deposition compared to CBDL rats (Figure 4-2C.b).

\subsubsection{M6P-HPMA-TFO Inhibits Profibrogens}

TGF- $\beta 1$ is a potent fibrogenic cytokine believed to regulate fibrosis. Therefore, we next determined the effect of M6P-HPMA-TFO on hepatic TGF- $\beta 1$ expression by RT-PCR. Hepatic TGF- $\beta 1$ expression after CBDL was dramatically up-regulated. However, TFO and M6P-HPMA-TFO significantly decreased hepatic TGF- $\beta 1$ gene expression (Figure 4-3A). M6P-HPMA-TFO injected group showed even less TGF- $\beta 1$ gene expression. TGF- $\beta 1$ protein expression in the liver was also examined by Western blot. As shown in Figure 4-3D, TGF- $\beta 1$ protein expression reduced markedly in M6P-HPMA-TFO treated group than TFO group.

\subsubsection{Inhibition of Collagen Intrinsic Degradation}

TIMP-1 is the tissue inhibitor of metalloproteinases which can inhibit most interstitial collagenases and matrix metalloproteinases (MMPs), which have the ability to degrade the normal liver matrix. After CBDL, there was a significant increase in TIMP-1 expression in the liver, but its expression significantly decreased by TFO and M6P-HPMA-TFO treatment (Figure 4-3B). M6P-HPMA-TFO treatment showed better reduction in TIMP-1 level compared to TFO treatment (Figure 4-3B).

\subsubsection{Inhibition on Myofibroblast Activation}

The presence of $\alpha$-smooth muscle actin ( $\alpha$-SMA) positive cells has been reported to increase in the fibrotic liver. ${ }^{222}$ Therefore, we determined $\alpha$-SMA concentration in mRNA level before and after treatment. There was significant increase in $\alpha$-SMA mRNA level at two weeks after CBDL only (Figure 4-3C). Both TFO and M6P-HPMA-TFO treatment significantly decreased $\alpha$-SMA mRNA synthesis in CBDL rats. M6P-HPMA-TFO injection in alternative day for two weeks decreased $\alpha$-SMA expression even less (Figure 4-3C). $\alpha$-SMA gene expression was also determined at protein levels by Western blot in whole liver lysis showed the same trend as RT-PCR (Figure 4-3D).

There was significant increase in $\alpha$-SMA immunostaining in CBDL liver forming whorls of layers (onion skinning) around the proliferated bile ducts (Figure 4-4). 


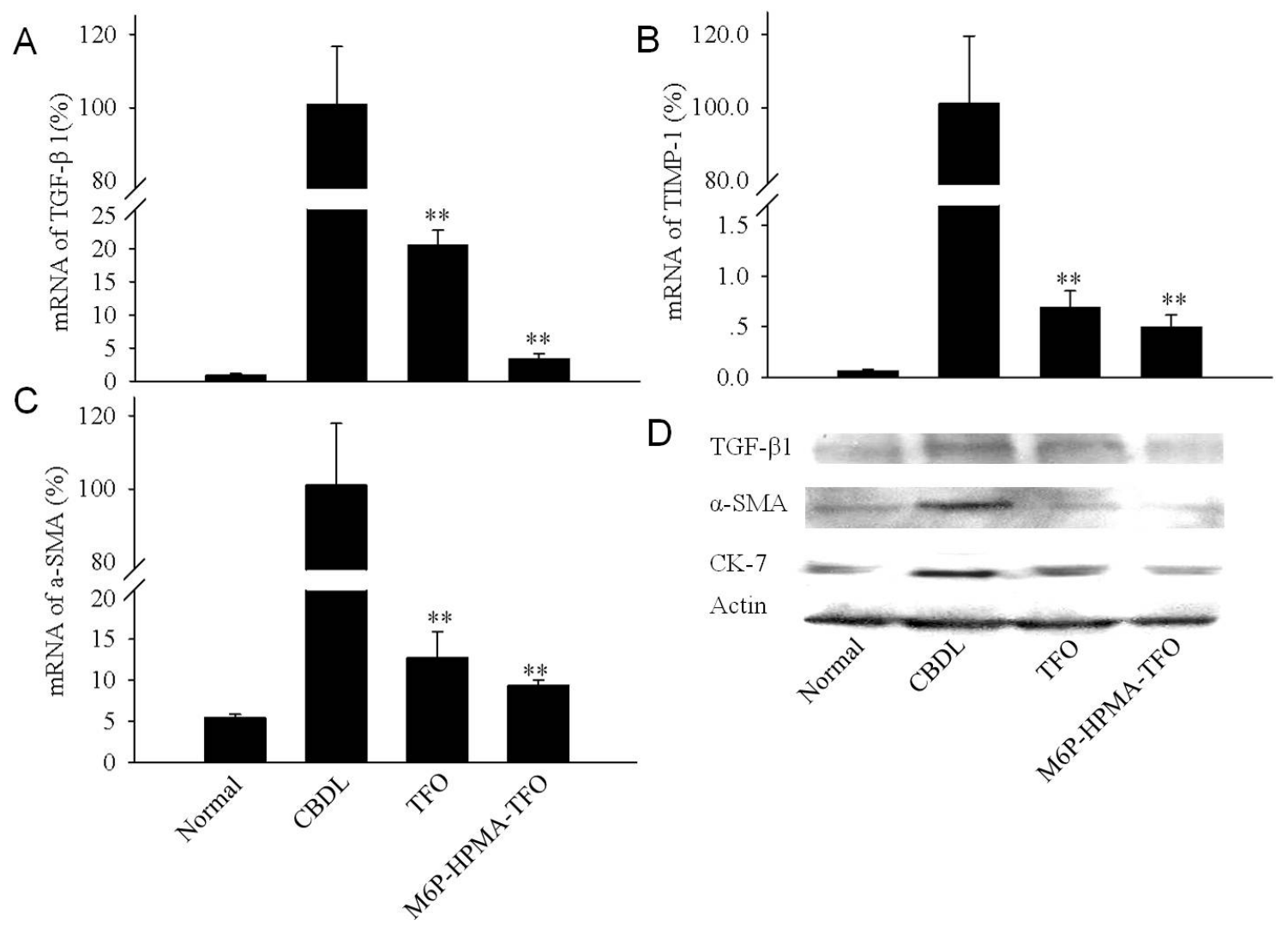

Figure 4-3 mRNA and protein expression level screening of fibrotic related factors. A) mRNA level of TGF- $\beta 1$, B) mRNA level of TIMP1, C) mRNA level of $\alpha$-SMA, D) protein expression of TGF- $\beta 1, \alpha-\mathrm{SMA}$ and $\mathrm{CK}-7$. 


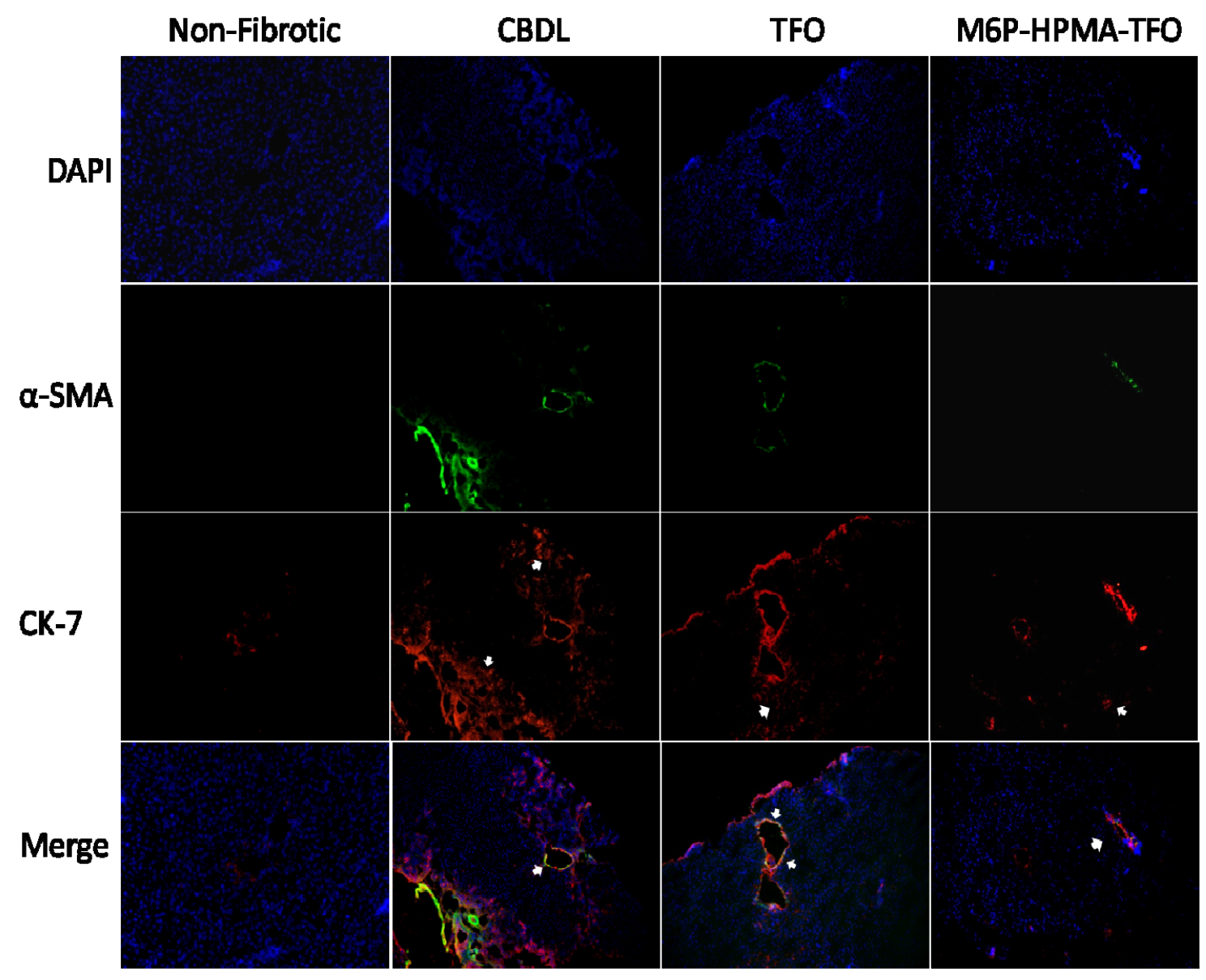

Figure 4-4 Co-localization of $\alpha$-SMA and CK-7. M6P-GFLG-HPMA-GFLG-TFO group showed higher expression of CK-7 and $\alpha$-SMA. 
Furthermore, two weeks after CBDL, CK-7 expression in hepatocytes was up-regulated but decreased with TFO and M6P-HPMA-TFO treatment (arrows). CK-7 is up-regulated in injured hepatocytes before they go to apoptosis even though it is always expressed in the epithelia around the bile ducts. ${ }^{223}$ M6P-HPMA-TFO treatment attenuated CK-7 expression in hepatocytes more than TFO treatment, which may imply the function of damaged hepatocytes was at least partially restored (Figure 4-4). Western blot showed the same trend (Figure 4-3D). Besides hepatocytes, clear co-localization of CK-7 and myofibroblast marker $\alpha$-SMA was observed in biliary epitheliums. M6P-HPMA-TFO injection decreased this co-localization, suggesting higher inhibition ability on myofibroblastic phenotypic transition compared to only TFO treatment (Figure 4-4).

\subsubsection{Effects on Serum Fibrotic and Pro-inflammatory Markers}

CBDL caused jaundice in rats, leading to the urine color change to dark yellow several days after CBDL. The level of traditional liver disease markers such as ALT and AST were determined. ALT and AST levels were higher in CBDL group. However, administration of TFO and M6P-HPMA-TFO resulted in decreased levels of ALT and AST. M6P-HPMA-TFO group showed even less ALT and AST levels compared to TFO group (Figure 4-5A \& B).

Jaundice comes from the decreased processing of total bilirubin. The amount of bilirubin was reduced in therapeutic groups, especially in M6P-HPMA-TFO group (Figure 4-5C), which may indicate the treatment makes the liver more robust and prevents it from being continually damaged by the retro-flowing bile.

There was a significant decrease in TNF- $\alpha$, one pro-inflammatory factor, level in treatment group. When CBDL rats were treated with TFO and M6P-HPMA-TFO, M6P-HPMA-TFO treatment control the inflammation better than free TFO (Figure $4-5 \mathrm{D})$.

\subsubsection{Inhibition on Neutrophil Infiltration}

Bile duct obstruction causes neutrophilic inflammation of the liver and leads to liver fibrosis. ${ }^{224}$ Activated neutrophils up-regulate adhesion molecules receptor (CD11/CD18)

on its membrane surface, then adheres to endothelial cells of hepatic sinusoids. ${ }^{225}$ In this study, CD 11 was used as a bio-marker to measure the neutrophil infiltration in fibrotic livers. The accumulated CD 11 staining indicated enhanced activated neutrophil accumulation in CBDL rats (Figure 4-6B). M6P-HPMA-TFO injection group (Figure 4-6D) decreased this accumulation more than free TFO group (Figure 4-6C). This phenomenon is partially due to the reduction of pro-inflammation, which was shown in the data of TNF- $\alpha$ level (Figure 4-5D). Increase in neutrophil infiltration is due to systemic or local exposure to pro-inflammatory mediators, such as TNF- $\alpha$, IL-1 and CXC chemokines. Another reason of the reduction of neutrophil infiltration may come from the 

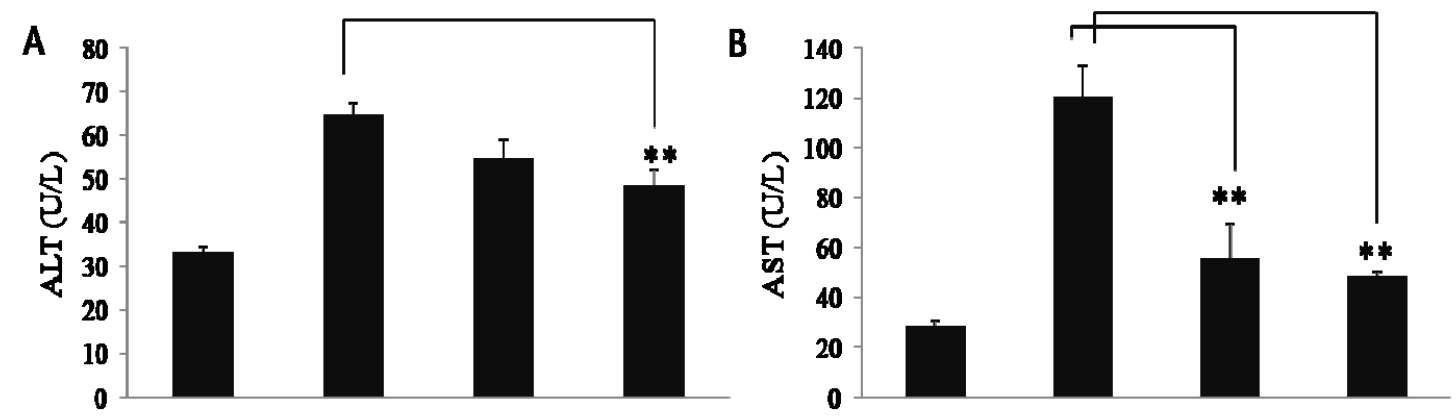

C
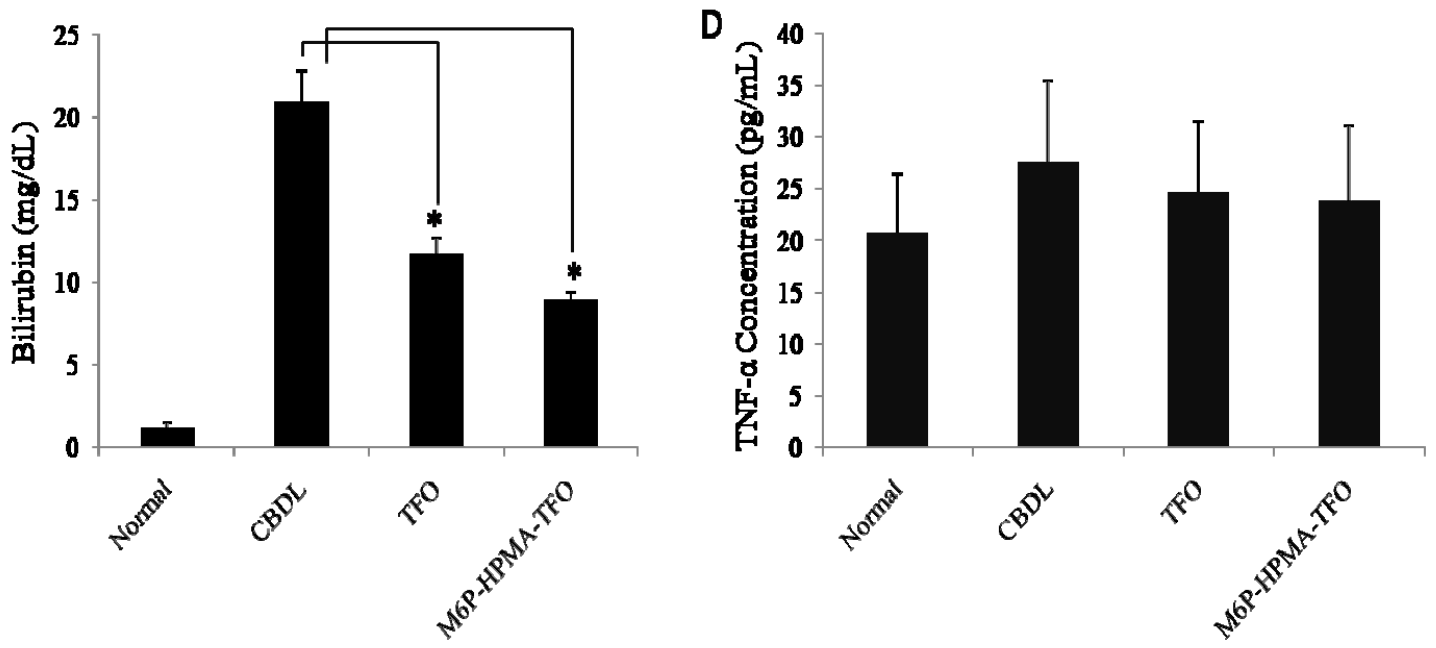

Figure 4-5 Blood chemistry assay. A) ALT, B) AST, C) Bilirubin, D) TNF- $\alpha$. 

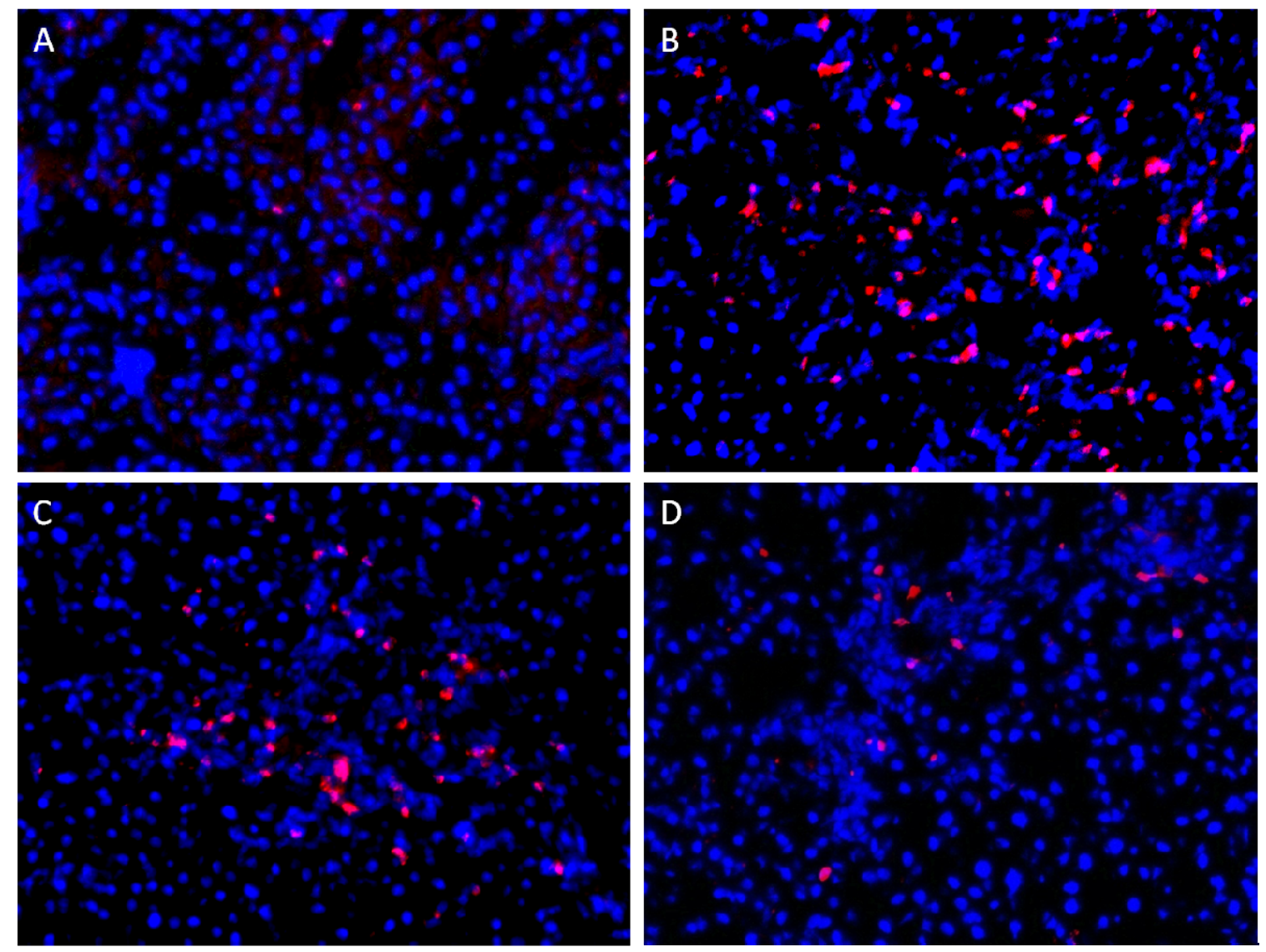

Figure 4-6 CD-11 staining to indicate neutrilphil infiltration. A) non-fibrotic rat, B) CBDL rat, C) free TFO group, D) M6P-GFLG-HPMA-GFLG-TFO group. 
protection of HPMA to TFO to prevent or relieve extra immune response compared to free TFO after systemic administration.

\subsection{Discussion}

Liver fibrosis is characterized by excessive production of collagen, primarily by activated HSCs. Therefore, we recently conjugated TFO targeting type I collagen transcription to M6P-HPMA for targeted delivery to HSCs after systemic administration.

Triplex formation happens at sequences containing a stretch of pyrimidines on one DNA strand and complementary purines on the other DNA strand. TFOs containing C and $\mathrm{T}$ nucleotides bind in a parallel direction to the purine-containing strand of double stranded DNA, whereas TFOs containing G and A nucleotides bind in an anti-parallel orientation. This combination is involved in gene regulation in many areas, including transcription, replication, repair and recombination. ${ }^{41,226,227}$ We demonstrated that TFO can form triplex with type I collagen promoter in genomic DNA to inhibit the transcription of this protein. ${ }^{34}$ This approach enhances the silence efficiency compared antisense strategies because it shut down the original source of type I collagen. However, to enter the nuclear, it is necessary for our TFOs to reach the cytoplasm of HSCs in high concentration in cytoplasm in HSCs, the target cells. Specific carriers and ligands were taken into account, such as BSA and M6P. BSA showed enhanced delivery efficiency, however, even though already conjugated to M6P, this conjugate still has immune and size problems. ${ }^{38}$ Therefore, HPMA copolymer, as an alternate carrier was considered. ${ }^{27}$

HMPA is a non-immunogenic copolymer containing a lysosomal degradable GFLG tetrapeptide spacer, facilitating TFO release from the conjugate for nuclear translocation to inhibit collagen transcription. Furthermore, we determined previously that TFO conjugation to M6P-HPMA has no adverse effect in its ability to triplex formation. ${ }^{27}$ M6P-HPMA-TFO also inhibited type I collagen synthesis in HSC-T6 cell line. The difference in TNF- $\alpha$ level is not significant between TFO group and M6P-HPMA-TFO group after two weeks short term therapeutics. Neutrophil infiltration was much less in M6P-HPMA-TFO group compared to TFO injected group. This result implies that reduced neutrophil accumulation might not only come from less exposure to pro-inflammatory factors, which were thought as one reason for neutrophil infiltration. The extra relief of neutrophil infiltration may be due to the protection from conjugated HPMA to TFO to prevent or at least lighten the attack from immune system to the intruder, free TFO.

Our short term experiments also unveiled that the continual TFO function helps the damaged hepatocytes to recover. CK-7 is expressed in the bile duct epithelia of both healthy and hepatic fibrotic rats. ${ }^{228}$ However, CK-7 is only expressed in injured hepatocytes. ${ }^{223}$ The staining of CK-7 in liver parenchyma were reduced a lot especially in M6P-HPMA-TFO treated group (Figure 4-4). This result indicates damaged hepatocytes recovered because of the inhibition of excessive collagen synthesis whereas staining of 
the hepatocytes by CK-7 predicts subsequent progression of fibrosis. M6P-HPMA-TFO group showed higher effects because of the efficient targeted delivery of TFO.

Cell adhesion molecules facilitate leukocyte-endothelial cell interactions and coordinate many other cell interactions through a variety of adhesion receptors, including integrins, selectins, immunoglobulin-like molecules and cadherins. ${ }^{229-231}$ These cell adhesion molecules regulate leukocyte infiltration through a mechanism of ligand binding. This typically includes qualitative changes in receptor surface expression and transient activation of leukocyte infiltration. ${ }^{232,233} \mathrm{CD}-11$ is $\alpha$ component of various integrins and therefore mediates leukocyte adhesion. The most frequently observed $\beta$ component is $\mathrm{CD} 18$ ( $\beta 2)$. CD11b/CD18 was initially described as receptors which facilitate the adhesion of T cells, expressing CD11a/CD18 too. ${ }^{233}$ Neutrophil infiltration into the inflammatory site and subsequent release of toxic factors by these cells is partly responsible for liver fibrosis. There was strong staining for CD11 in CBDL rats (Figure 4-6B), which indicated enhanced neutrophil accumulation. M6P-HPMA-TFO injection group (Figure 4-6D) relieve this infiltration more than free TFO group (Figure 4-6C). Besides systemic or local exposure to pro-inflammatory mediators, such as TNF- $\alpha$ (Figure 4-5D), neutrophil infiltration reduction may results from the protection of HPMA to TFO to decrease immune response.

Generally, M6P-HPMA-TFO enhanced the efficacy of TFO in hepatic fibrosis treatment. 


\section{CHAPTER 5. TGF- $\beta 1$ GENE SILENCING FOR TREATING LIVER FIBROSIS}

\subsection{Introduction}

Liver fibrosis is the excessive accumulation of extracellular matrix (ECM) proteins resulting from chronic liver damage. In general, it is an imbalance between the synthesis and degradation of ECM. In the presence of chronic liver injuries, hepatic stellate cells (HSCs) become activated and transform to proliferative myofibroblast-like cells, which account for major source of ECM expression. ${ }^{220,234}$

There is no standard treatment for liver fibrosis and therefore the effective anti-fibrotic medicines are needed urgently. ${ }^{220,235}$ Among many inflammatory cytokines involved in liver fibrosis, TGF- $\beta 1$ appears to be the most important (Figure 5-1) ${ }^{28,220}$ Cytokines of TGF family affect a variety of cellular processes, including differentiation, proliferation, apoptosis and migration. Among them, TGF- $\beta 1$ is the most potent profibrogenic factor involved in initiation and maintenance of fibrogenesis in the liver. ${ }^{236,237}$ Stimulation of activated HSC by TGF- $\beta 1$ is believed to be the key fibrogenic response in liver fibrosis because of following evidences: $i)$ higher TGF- $\beta 1$ expression in activated HSC; ii) potency of TGF- $\beta 1$ to up-regulate ECM expression; iii) higher expression of TGF- $\beta$ receptors on HSC; iv) TGF- $\beta 1$ increases the expression of tissue inhibitor of metalloproteinases 1 (TIMP-1). ${ }^{236-238}$ TGF- $\beta 1$ in the liver is also secreted by hepatocytes, kupffer cells, stellate cells, endothelial cells and infiltrating mononuclear cells. ${ }^{236}$

Strategies aimed at disrupting TGF- $\beta 1$ expression or signaling pathways are extensively being investigated because blocking this cytokine may not only inhibit matrix production, but also accelerate its degradation. ${ }^{239}$ Animal experiments using different strategies to block TGF- $\beta 1$ have demonstrated significant anti-fibrotic effect for liver fibrosis. ${ }^{44,237,240-244}$ RNA interference (RNAi) is the phenomenon in which siRNA of 21-23 nt in length silences a target gene by binding to its complementary mRNA and triggering its degradation. Potent knockdown of the target gene with high sequence specificity makes siRNA a promising therapeutic strategy. ${ }^{245}$ Compared to antisense oligonucleotides, neutralizing antibodies and soluble TGF- $\beta$ receptors strategies, siRNA has the potent knockdown of the target gene with high sequence specificity. siRNAs targeting other pathways have been proven effective in treating liver fibrosis ${ }^{246-249}$ and renal fibrosis. ${ }^{147}$ There are three ways to deliver siRNA: synthetic duplex, plasmid and viral vectors. While viral vectors give high transfection efficiency, their immune reactions limit their application in therapeutics. Plasmid DNA complexes with cationic liposomes to form particle in the size of $250 \mathrm{~nm}$, which may not pass through the sinusoidal gaps, because these fenestrae get lost during liver fibrosis. In contrast, low molecular weight of synthetic duplex siRNA is expected to pass through the sinusoidal gaps of fibrotic liver and thus it may be an ideal candidate for treating liver fibrosis.

In this study, we screened ten chemically synthesized siRNAs targeting different regions of TGF- $\beta 1$ mRNA and then converted the most potent siRNA sequences into 


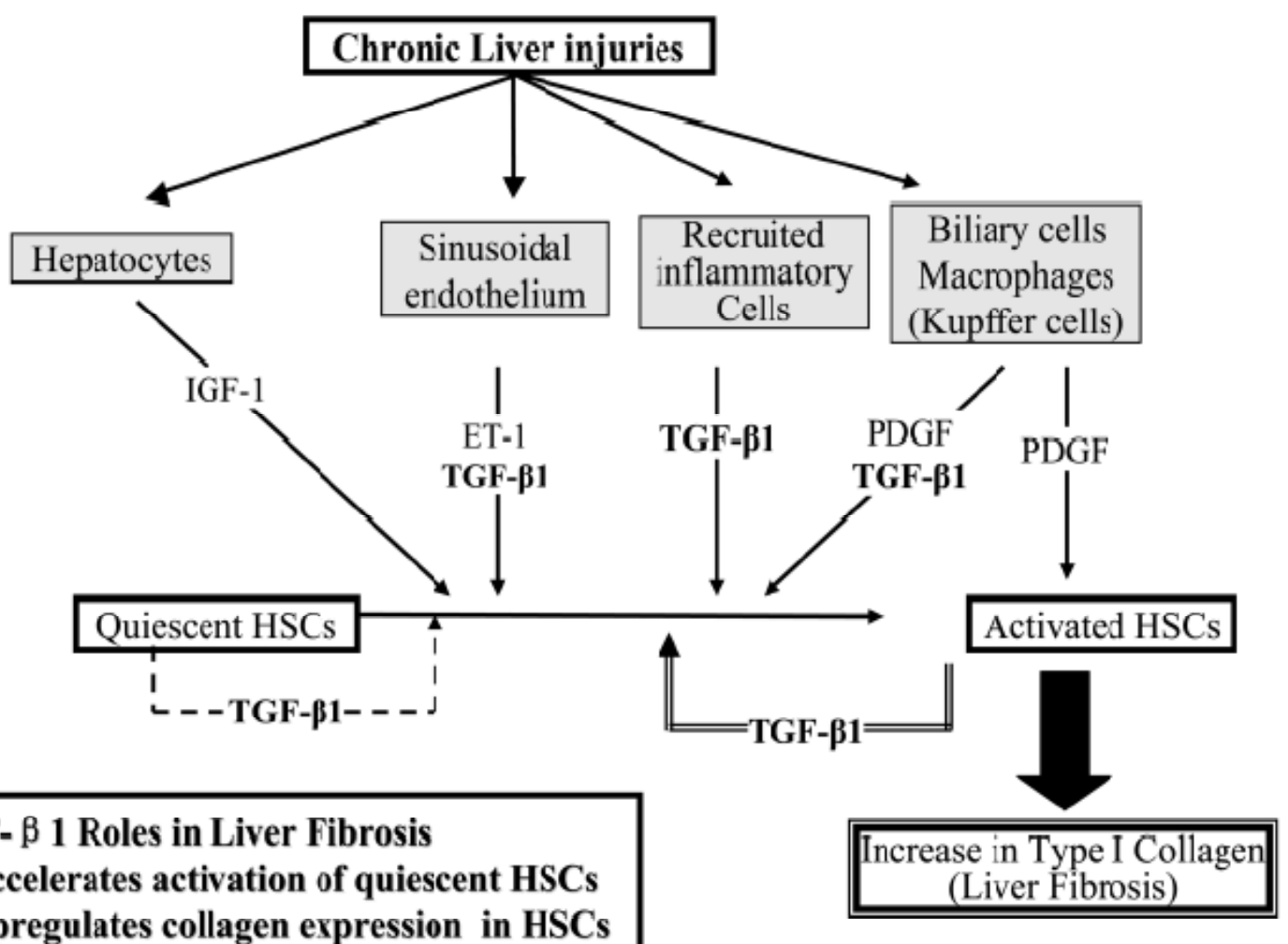

Figure 5-1 Role of TGF- $\beta 1$ in liver fibrosis. TGF- $\beta 1$ is the most potent single profibrogenic factor involved in initiation and maintenance of fibrogenesis in the liver. TGF- $\beta 1$ accelerates activation of quiescent hepatic stellate cells (HSCs), upregulates collagen expression, and decreases collagen degradation. 
shRNA via cloning into pScilence 1.0 vector. Both synthetic siRNA and shRNA expression plasmids were tested in HSC-T cells for gene silencing and therapeutic efficacy.

\subsection{Materials and Methods}

\subsubsection{Materials}

Trizol was purchased from Invitrogen Corporation (Carlsbad, CA). pScience1.0 was purchased from GenScript Corporation (Piscataway, NJ). Bovine serum albumin (BSA) (fraction V, purity $>98 \%$ ) was purchased from USB Corporation (Cleveland, $\mathrm{OH}$ ). Dulbecco's modified eagle's medium (DMEM), penicillin G $(5000 \mathrm{U} / \mathrm{mL})$, Trypsin-EDTA, Trizol, DNase I, Lipofectamine 2000 were purchased from Invitrogen Life Technologies (Carlsbad, CA). Restriction enzymes were purchased from New England Biolabs (Ipswich, MA). SYBR Green-1 dye universal master mix and Multiscript reverse transcriptase were purchased from Applied Biosystems, Inc. (Foster City, CA). TGF- $\beta 1$ ELISA kit was purchased from R\&D Systems, Inc. (Minneapolis, $\mathrm{MN}$ ). TNF- $\alpha$ and IL-1 $\beta$ ELISA kits were purchased from eBioscience, Inc. (San Diego, CA).

\subsection{2. $\quad$ siRNA Design and Synthesis}

Ten siRNA sequences targeting TGF- $\beta 1$ mRNA (Accession\#: NM_021578) and one control siRNA were designed using BLOCK-iTTM RNAi Designer and purchased from Invitrogen Corporation (Carlsbad, CA) and their sequences are listed in Table 5-1. These siRNAs are of 19-21 nt with a 2 thymidine deoxnucleotide overhangs at the 3 '-end. All designed siRNA sequences were blasted against the rat genome database to eliminate cross-silence phenomenon with non-target genes.

\subsubsection{Design and Construction of shRNA Expression Plasmids}

Following screening of different siRNA sequences, two effective and one control siRNA sequences were converted into shRNA (Table 5-2). pshRNA-769, pshRNA-1033 and control vectors were constructed using pScilence 1.0 vector, which carries shRNA expression cassette under the control of U6 promoter (GenScript Corporation, Piscataway, NJ). These shRNAs contain two complementary oligonucleotides that were annealed to form a double-stranded DNA for ligation into pScilence 1.0 vector at corresponding sites under U6 promoter using T4 DNA ligase for $2 \mathrm{~h}$ at $25^{\circ} \mathrm{C}$. Following transformation in Top10 supercompetent cells and amplification in broth media, plasmids were purified using QIAGEN Plasmid Kit. 
Table 5-1 Predesigned siRNAs for rat TGF- $\beta 1$ using BLOCK-iT RNAi designer.

\begin{tabular}{cc}
\hline Start & Sequence (DNA) \\
\hline 297 & GCCAGATCCTGTCCAAACT \\
448 & GGACTACTACGCCAAAGAA \\
499 & CGCAATCTATGACAAAACC \\
640 & GCAACACGTAGAACTCTAC \\
769 & GAACCAAGGAGACGGAATA \\
888 & GCACCATCCATGACATGAA \\
1033 & GCAGCTGTACATTGACTTT \\
1036 & GCTGTACATTGACTTTAGG \\
1167 & CCCTCTACAACCAACACAA \\
1245 & TCTACTACGTGGGTCGCAA \\
\hline
\end{tabular}


Table 5-2 Sequences of shRNA against different target regions of TGF- $\beta 1$.

\begin{tabular}{|c|c|}
\hline shRNA insert & Sequence \\
\hline Control shRNA & $\begin{array}{l}\text { Antisense: 5'-ACTTCATAAGGCGCATGCTTTCAAGAGAAGCATGCGCCTTATGAAGTTTTTTT-3' } \\
\text { Sense: 5'-AATTAAAAAAACTTCATAAGGCGCATGCTTCTCTTGAAAGCATGCGCCTTATGAAGTGGCC-3' }\end{array}$ \\
\hline shRNA-769 & $\begin{array}{l}\text { Antisense: 5'-GAACCAAGGAGACGGAATATTCAAGAGATATTCCGTCTCCTTGGTTCTTTTTT-3' } \\
\text { Sense: 5'-AATTAAAAAAGAACCAAGGAGACGGAATATCTCTTGAATATTCCGTCTCCTTGGTTCGGCC-3' }\end{array}$ \\
\hline shRNA-1033 & $\begin{array}{l}\text { Antisense: 5'-GCAGCTGTACATTGACTTTTTCAAGAGAAAAGTCAATGTACAGCTGCTTTTTT-3' } \\
\text { Sense: 5'-AATTAAAAAAGCAGCTGTACATTGACTTTTCTCTTGAAAAAGTCAATGTACAGCTGCGGCC-3' }\end{array}$ \\
\hline
\end{tabular}




\subsubsection{Transfection of SiRNA and ShRNA}

Immortalized rat hepatic stellate cells (HSC-T6), kindly provided by Dr Scott Friedman, Mount Sinai School of Medicine, New York, were seeded in 6-well plates at a density of $11 \times 10^{5}$ cells $12 \mathrm{~h}$ before transfection. siRNA duplexes were mixed with $4 \mu \mathrm{L}$ Lipofectamine 2000 in $200 \mu \mathrm{L}$ Opti-MEM I medium for $20 \mathrm{~min}$ at room temperature to allow complex formation. The transfection mixture was then added to each plate with 1.3 $\mathrm{mL}$ fetal bovine serum (FBS) free Dulbecco's Modified Eagle's Medium (DMEM) at a concentration of $150 \mathrm{nM}$ after washing cells with PBS. After $24 \mathrm{~h}$ of incubation, $0.5 \mathrm{~mL}$ DMEM containing 10\% FBS was added and incubated for another $24 \mathrm{~h}$.

In case of shRNA expression plasmids, cells were transfected with shRNA expression vectors at doses of $1 \mu \mathrm{g} /$ well. The shRNA plasmids were mixed with Lipofectamine 2000 in $250 \mu \mathrm{L}$ Opti-MEM I medium for $20 \mathrm{~min}$ at room temperature to allow complex formation. The transfection mixture was then added to each plate with $2 \mathrm{~mL}$ fetal bovine serum (FBS) free Dulbecco's Modified Eagle's Medium (DMEM). After $8 \mathrm{~h}$ of incubation, $200 \mu \mathrm{L}$ FBS was added and incubated for another $42 \mathrm{~h}$. The supernant was collected for western blot assay. Results were normalized with total protein content of cells.

\subsubsection{Real Time RT-PCR}

Total RNA was isolated from the cell pellets using Trizol reagent. Total RNA (1 $\mu \mathrm{g})$ was converted to cDNA using MultiScribe Reverse Transcriptase Reagent and random hexamers (Applied Biosystems, Inc., Branchburg. NJ). One hundred nanograms of the cDNA was amplified by Real Time PCR using SYBR Green-1 dye universal master mix on ABI Prism 7700 Sequence Detection System (Applied Biosystems, Inc., Foster City, CA). We used the following primers: (i) TGF- $\beta 1$ : Forward: 5'-CATCCATGACAT GAA CCG ACC CTT-3' and reverse: 5'-ACAGAAGTTGGCATGGTAGCCCTT-3'; (ii) TIMP-1: forward: 5'-CCTCTGGCATCCTCTTGTTGCTAT-3'and reverse:

5'-CATTTCCCACAG CGTCGAATCCTT-3'; (iii) $\alpha 1$ (I) collagen mRNA: Forward: 5'-TGGTCCCAAAGGTTC TCC TGG T-3' and reverse:

5'-TTAGGTCCAGGGAATCCCATCACA-3'; and (iv) $\alpha$-SMA: forward:

5'-ACAACGTGCCTATCTATG AGGGCT-3' and reverse:

5'-AGCGACATAGCACAGCTTCTCCTT-3'. We used 18S ribosomal RNA as an internal control: forward: 5'-GTCTGTGATGCCCTTAGATG-3' and reverse: 5'-AGCTTATGACCCGCACTTAC-3'. To confirm PCR specificity, the PCR products were subjected to a melting-curve analysis. Comparative threshold (CT) method was used for calculating the relative amount of mRNA of treated sample to control samples (Figure $5-2){ }^{250}$

In case of in vivo evaluation in fibrotic mice, we used the following primers: (i) TGF- $\beta 1$ : forward 5'-CATCCATGACATGAACCGGCC-3' and reverse: 5'-ACAGAAGTTGGCATGGTAGCCCTT-3'; (ii) TIMP-1: forward: 


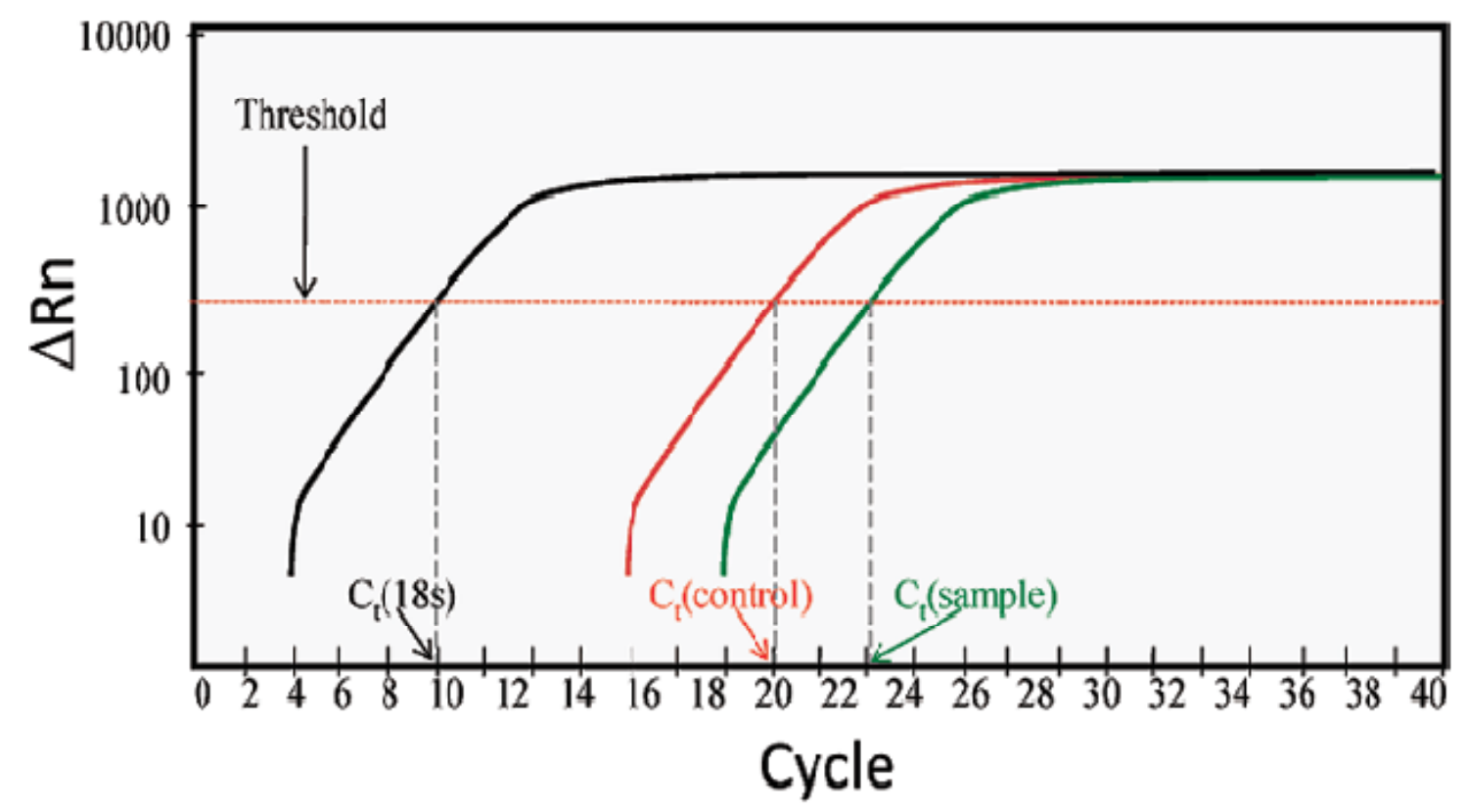

Figure 5-2 The real time PCR plot. Black curve is the intrinsic reference (18s). Red curve is the control group. Green curve is the group with treatment. The following two equations are used in calculating the silencing effect.

$\Delta \Delta \mathrm{C}_{\mathrm{t}}=\left[\mathrm{C}_{\mathrm{t}}(\right.$ sample $\left.)-\mathrm{C}_{\mathrm{t}}(18 \mathrm{~s})\right]-\left[\mathrm{C}_{\mathrm{t}}(\right.$ control $\left.)-\mathrm{C}_{\mathrm{t}}(18 \mathrm{~s})\right]$

Silencing effect $(\%$ of remaining mRNA $)=100 \times \frac{1}{2^{\Delta \Delta C t}}$ 
5'-CATCTGGCATCCTCTTGTTGCTAT-3'and reverse:

5'-CATTTCCCACAGCCTTGAATCCTT-3'; and (iii) $\alpha$-SMA were

5'-ACAACGTGCCTATCTATGAGGGCT-3' and reverse:

5'-AGCGACATAGCACAGCTTCTCCTT-3'. All other conditions were the same.

\subsubsection{Western Blot Assay}

Proteins in the cell culture medium were purified using Microcon YM-30 columns (Millipore) and then lysed using $2 \times$ Laemmli sodium dodecyl sulfate (SDS) sample buffer containing $100 \mathrm{mM}$ Tris, pH 6.8, $200 \mathrm{mM}$ dithiothreitol (DTT), 4\% SDS, 20\% glycerol and $0.2 \%$ bromophenol blue. To immunodetect cellular proteins, cells were lysed directly with $1 \times$ Laemmli SDS sample buffer. The lysate was then boiled at $100^{\circ} \mathrm{C}$ for $5 \mathrm{~min}$ and subjected to $6 \%$ or $10 \%$ SDS-polyacrylamide (SDS-PAGE) gel electrophoresis and subsequent transfer to Immobilon polyvinylidene fluoride (PVDF) membrane (Millipore). After blocking with 5\% nonfat dried milk in $1 \times$ PBST containing $0.05 \%$ Tween-20 in PBS for $1 \mathrm{~h}$ at room temperature, the membranes were incubated with rabbit anti-rat type I collagen (Calbiochem), TGF- $\beta 1$ (Santa Cruz); $\beta$-actin (Santa Cruz) primary antibodies for $16 \mathrm{~h}$ at $4^{\circ} \mathrm{C}$. Membrane was then incubated with horseradish peroxidase-conjugated goat anti-rabbit secondary antibody (Santa Cruz) for $1 \mathrm{~h}$ at room temperature. Target proteins were detected by enhanced chemiluminescence (ECL, GE Healthcare).

\subsubsection{ELISA Assay of TNF- $\alpha$ and IL-1 $\beta$}

Cells were transfected with shRNA expression vectors at doses of $1 \mu \mathrm{g} / \mathrm{well}$. The shRNA plasmids were mixed with $8 \mu \mathrm{L}$ Lipo-lin in $250 \mu \mathrm{L}$ Opti-MEM I medium for 20 min at room temperature to allow complex formation. The transfection mixture was then added to each plate with $2 \mathrm{~mL}$ fetal bovine serum (FBS) free Dulbecco's Modified Eagle's Medium (DMEM). After $3 \mathrm{~h}$ of incubation, $200 \mu \mathrm{L}$ FBS was added and incubated for another $42 \mathrm{~h}$. Then the supernant of the cultured cells were collected. The concentration of TNF- $\alpha$ and IL-1 $\beta$ were measured using enzyme-linked immunosorbent assay (ELISA) (eBioscience, USA), according to the manufacturer's protocol. Briefly, Corning Costar 96 well ELISA plate was coated with $100 \mu \mathrm{L} /$ well of capture antibody in Coating Buffer. Sealed the plate and incubated overnight at $4{ }^{\circ} \mathrm{C}$. Then aspirated wells and washed 5 times with $>250 \mu \mathrm{L} /$ well Wash Buffer. Blocked wells with $200 \mu \mathrm{L} /$ well of $1 \times$ Assay Diluent. The plate was incubated at room temperature for 1 hour. Wells were aspirated and washed 5 times again. $100 \mu \mathrm{L} /$ well of sample was added to the appropriate wells and was incubated for 2 hours under room temperature. The wells were washed again. 100 $\mu \mathrm{L} /$ well of detection antibody was added and incubated for 1 hour at room temperature. The wells were washed and added $100 \mu \mathrm{L} /$ well of Avidin-HRP. The plate was sealed and incubated at room temperature for 30 minutes. After being washed for 7 times, each well was added by $100 \mu \mathrm{L} /$ well and incubated at room temperature for 15 minutes. Then 50 $\mu \mathrm{L}$ of Stop Solution was added to each well. The TNF- $\alpha$ and IL-1 $\beta$ concentration was determined spectrophotometrically at an absorbance of $450 \mathrm{~nm}$. 


\subsubsection{Persistence of TGF- $\beta 1$ Gene Silencing}

HSC-T6 cells were seeded in 6-well plates at a density of $11 \times 10^{5}$ cells $12 \mathrm{~h}$ before use. Both siRNA and shRNA were applied to the cells when the conflunce of the cells reached $40 \%$. $1 \mu \mathrm{g}$ siRNA duplexes or shRNA were mixed with $8 \mu \mathrm{L}$ Lipofectamine in $200 \mu \mathrm{L}$ Opti-MEM I medium for $20 \mathrm{~min}$ at room temperature to allow complex formation. The transfection mixture was then added to each plate with $2 \mathrm{~mL}$ FBS free DMEM at a concentration of $150 \mathrm{nM}$ for siRNA and $1 \mu \mathrm{g}$ per well for shRNA, both conditions are optimized condition for siRNA and shRNA transfection in this case, after washing cells with PBS. After $8 \mathrm{~h}$ of incubation, $200 \mu \mathrm{L}$ FBS was added and incubated for another $24 \mathrm{~h}, 48 \mathrm{~h}$ and $72 \mathrm{~h}$. In different time points, the cells are collected and total mRNA was isolated for Real Time PCR analysis.

\subsubsection{Statistical Analysis}

Data were expressed as the mean \pm standard deviation (SD). Difference between any two groups was determined by ANOVA. $p<0.05$ was considered statistically significant.

\subsection{Results}

\subsubsection{Effect of siRNA Sequences and Dose on TGF- $\beta 1$ Gene Silencing}

To examine the ability of siRNA to silence gene expression, we selected 19 bp siRNA duplexes targeting different regions of the rat TGF- $\beta 1$ genome (Table 5-1). These siRNA duplexes were transfected into rat HSC-T6 cells after complex formation with Lipofectamine 2000. As shown in Figure 5-3, TGF- $\beta 1$ gene silencing by siRNAs was sequence specific. Three siRNAs targeting TGF- $\beta 1$ start sites of 769, 886, 1033 and 1036 caused significant inhibition of TGF- $\beta 1$ expression, while the control siRNA had no effect on TGF- $\beta 1$ gene expression. Among all the siRNA sequences tested, siRNA-1036 showed the highest silencing effect up to $70 \%$, while siNA- 1033 showed up to $55 \%$ of gene silencing. Therefore, we selected siRNA-1036 and siRNA-1033 to determine the effect of siRNA dose on TGF- $\beta 1$ gene silencing. We also tested the pool of siRNAs targeting 769 and 1033, which also showed high TGF- $\beta 1$ gene silencing (Figure 5-3). As shown in Figures 5-4A and 5-4B, there was significant increase in the TGF- $\beta 1$ silencing effect as we increased the doses of siRNA-1033 and siRNA-1036 from $10 \mathrm{nM}$ to $150 \mathrm{nM}$, with siRNA-1036 showing the highest TGF- $\beta 1$ gene silencing at $150 \mathrm{nM}$.

We also determined the level of TGF- $\beta 1$ secreted into the cell culture medium by Western blot analysis at days 2 post-transfection of HSC-T6 cells with siRNAs targeting TGF- $\beta 1$ start sites of 769 and 1033 as well as the pool of these two siRNAs. As shown in Figure 5-4, the level of TGF- $\beta 1$ protein secreted in the culture medium was significantly lower than that of non-transfected and control-siRNA transfected samples. 


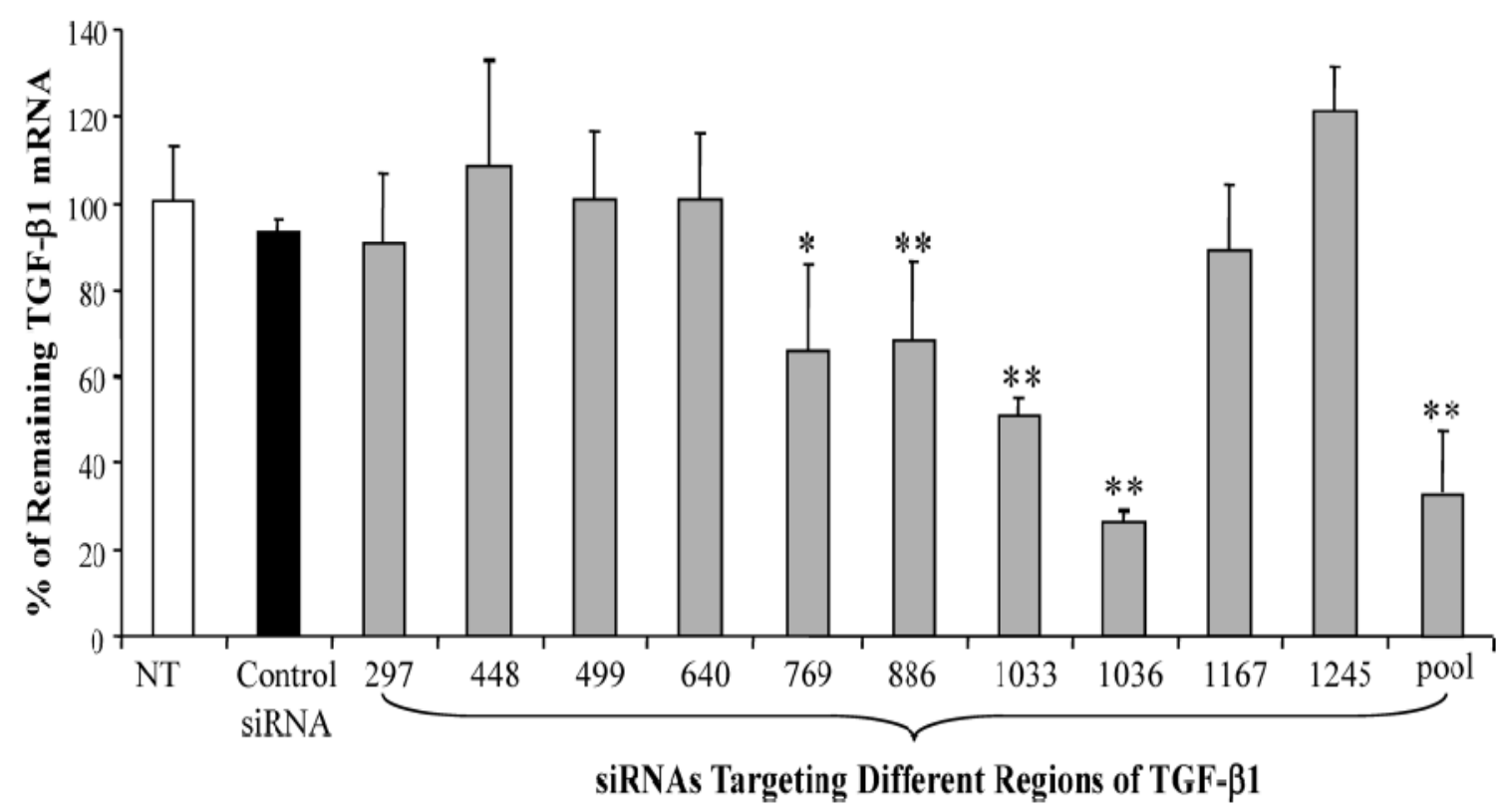

Figure 5-3 Effect of siRNA sequence on TGF- $\beta 1$ gene silencing. Ten different siRNAs targeting different regions of TGF- $\beta 1$ mRNA were transfected into HSC-T6 cells at a dose of $150 \mathrm{nM}$ after complex formation with Lipofectamine 2000 . TGF- $\beta 1$ gene silencing was determined by real time RT-PCR at $48 \mathrm{~h}$ post-transfection. Results were represented as the mean $\pm \mathrm{SD}(\mathrm{n}=3) .{ }^{*} \mathrm{p}<0.05, * * \mathrm{p}<0.01$. 

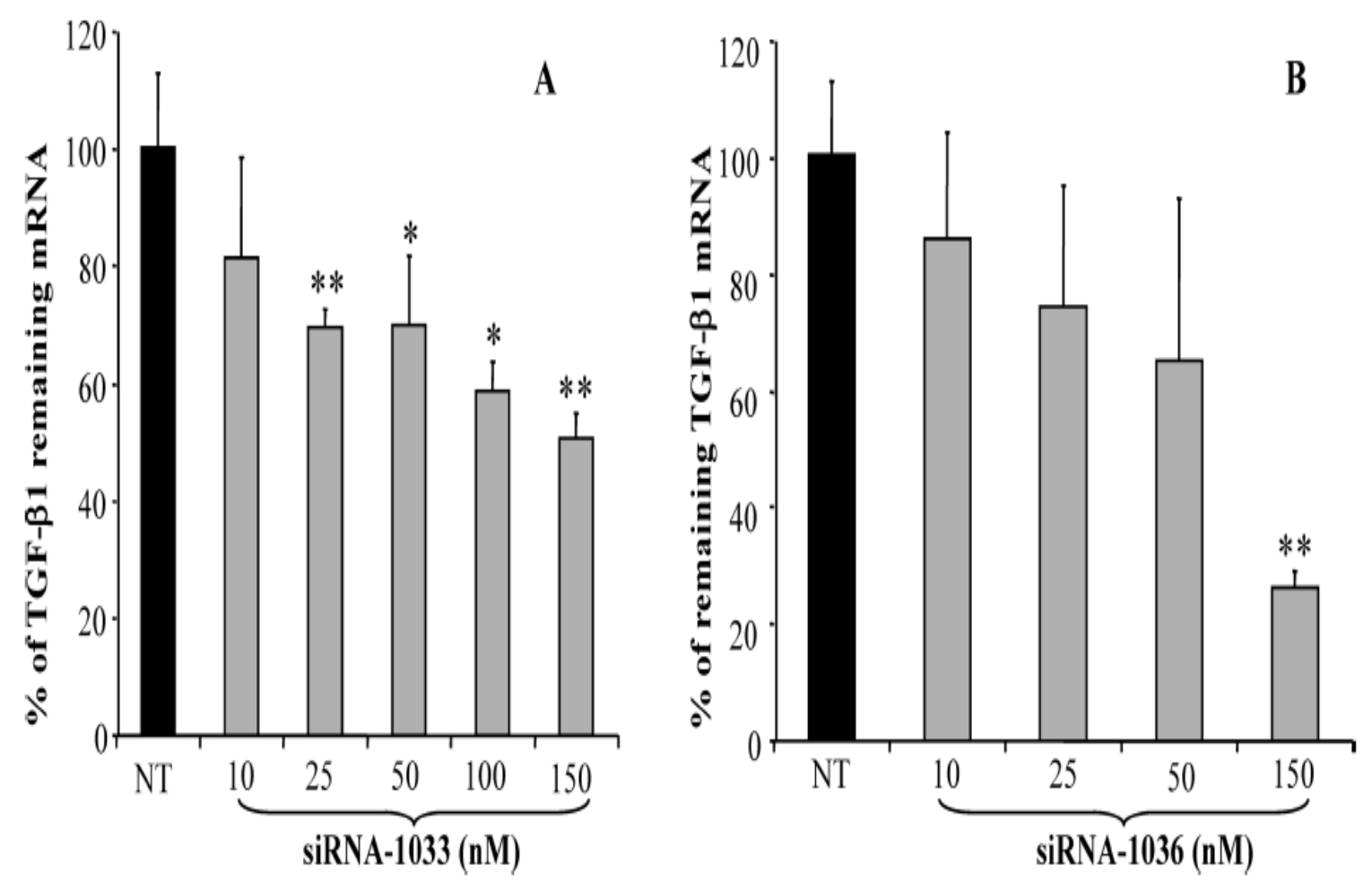

Figure 5-4 Effect of siRNA concentration on TGF- $\beta 1$ silencing. Lipofectamine 2000/siRNA complexes were transfected into HSC-T6 cells at doses of 10, 25, 50, 100, and $150 \mathrm{nM}$. TGF- $\beta 1$ gene silencing was determined by real time RT-PCR at $48 \mathrm{~h}$ post-transfection. Results were represented as the mean $\pm \mathrm{SD}(\mathrm{n}=3)$. ${ }^{*} \mathrm{p}<0.05,{ }^{* *} \mathrm{p}<0.01$. 


\subsubsection{Effect of TGF- $\beta 1$ Gene Silencing on TIMP-1 Expression}

TGF- $\beta 1$ is the key cytokine of fibrotic response to chronic liver injuries. It not only enhances ECM synthesis, but also inhibits ECM degradation by down-regulating matrix-degrading enzymes and inducing tissue inhibitor of metalloproteinases-1 (TIMP-1). ${ }^{251}$ Therefore, we measured TIMP-1 mRNA expression of HSC-T6 cells after transfection with TGF- $\beta 1$ siRNAs. As shown in Figure 5-5, both siRNA-1033 and siRNA-1036 inhibited TIMP-1 expression, while the control siRNA did not show any effect on TIMP-1 expression. These results suggest the similar inhibition profile of TIMP- 1 and TGF- $\beta 1$ after transfection of HSC-T6 cells with siRNA targeting TGF- $\beta 1$.

\subsubsection{Effect of siRNA on Type $\alpha(I)$ Collagen and $\alpha-S M A$ Expression}

Inhibition of TGF- $\beta 1$ should enhance the degradation of ECM, in which type I collagen is the main component. Therefore, we designed type $\alpha(\mathrm{I})$ collagen mRNA specific primers to determine the effect of TGF- $\beta 1$ siRNA on type $\alpha(\mathrm{I})$ collagen mRNA expression. At $150 \mathrm{nM}$, siRNA-1036 showed significant inhibition of type $\alpha(\mathrm{I})$ collagen mRNA (Figure 5-6A), while the control siRNA had little effect on $\alpha(\mathrm{I})$ collagen mRNA expression. We also determined the effect of TGF- $\beta 1$ gene silencing on type $\alpha(\mathrm{I})$ collagen protein expression by Western blot analysis of lysates of HSCT-6 cells after transfection with siRNA targeting TGF- $\beta 1$ start sites of 769 and 1033 as well as the pool of these two siRNAs (Figure 5-6B). Consistent with the RT-PCR results, there was significant decrease in collagen of siRNA-769, siRNA-1033 and siRNA pool, while there was no decrease in collagen concentration for the control-siRNA treated sample.

\subsubsection{Construction of shRNA Expression Vector}

Following screening of different siRNA sequences for TGF- $\beta 1$ gene silencing, we selected two potent siRNA sequences, which contain unique restriction sites at the 5' and 3 ' ends for cloning and a TTTTTT stretch in the sense to create the pol III terminal signal (Table 5-2). Then, these shRNA sequences were cloned into pRNAT-H1.1 and pSilencer1.0 as illustrated in Figure 5-7. The vector contains GFP under the control of CMV promoter, which can be used to track the transfection efficiency. pshRNA 769, pshRNA-1033 and pshRNA-1036 were confirmed by DNA sequencing using the following primers: forward: 5'-TAATACGACTCACTATAGGG-3' and reverse: 5'-TAGAAGGCACAGTCGAGG-3'.

\subsubsection{Effect of shRNA Expression on TGF- $\beta 1$ Silencing}

We determined the silencing effect of shRNA at different doses $(0.5$ and $1 \mu \mathrm{g})$ per well. shRNA expression plasmids showed higher TGF- $\beta 1$ gene silencing effect at $1 \mu \mathrm{g}$ than that at $0.5 \mu \mathrm{g}$ (Figure 5-8A). Among all the three shRNA expression plasmids we used, the plasmid encoding shRNA targeting 1033 and 1036 coding regions showed 


\section{TGF- $\beta 1>-\cdots$}

\section{$\beta$-Actin}

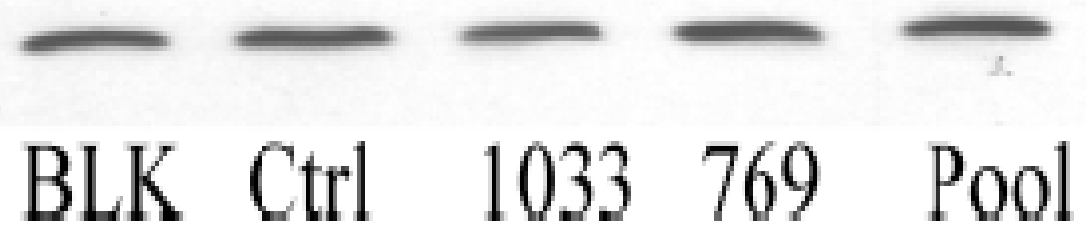

Figure 5-5 Western blot analysis for TGF- $\beta 1$ gene silencing after transfection of HSC-T6 cells with Lipofectamine/siRNA complexes. From left to right, no siRNA, control siRNA, siRNA-1033, siRNA-769 and pool of siRNA-1033 and siRNA-769. From top to bottom, TGF- $\beta 1$ and $\beta$-Actin. 


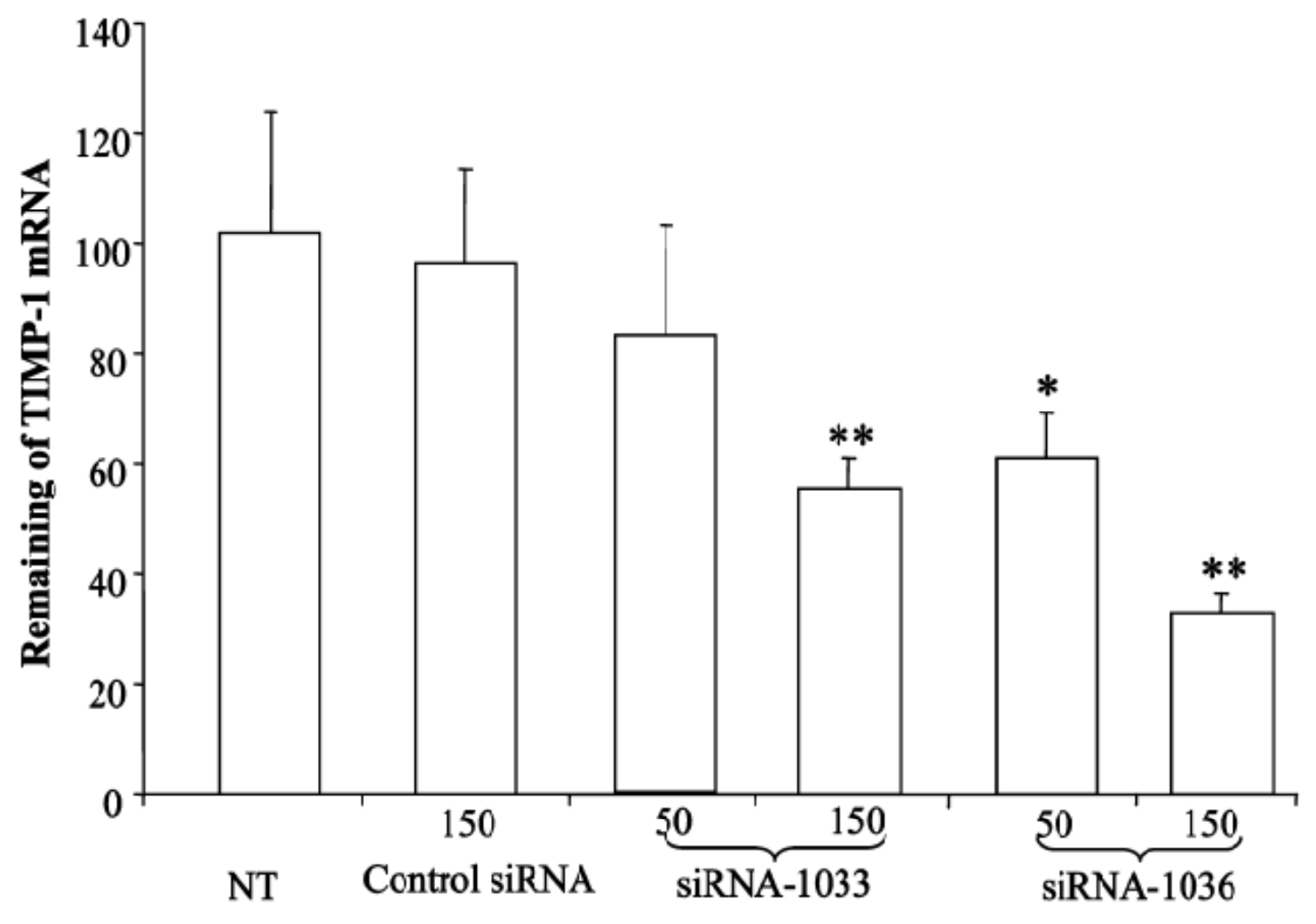

Figure 5-6 Effect of TGF- $\beta 1$ gene silencing on TIMP-1 gene expression after transfection of HSC-T6 cells with Lipofectamine 2000/siRNA complexes. At $48 \mathrm{~h}$ posttransfection, cells were harvested, total RNA was extracted, and TIMP-1 gene expression was determined at mRNA level using real time RT-PCR. Results were represented as the mean $\pm \mathrm{SD}(\mathrm{n}=3) .{ }^{*} \mathrm{p}<0.05,{ }^{* *} \mathrm{p}<0.01$. 


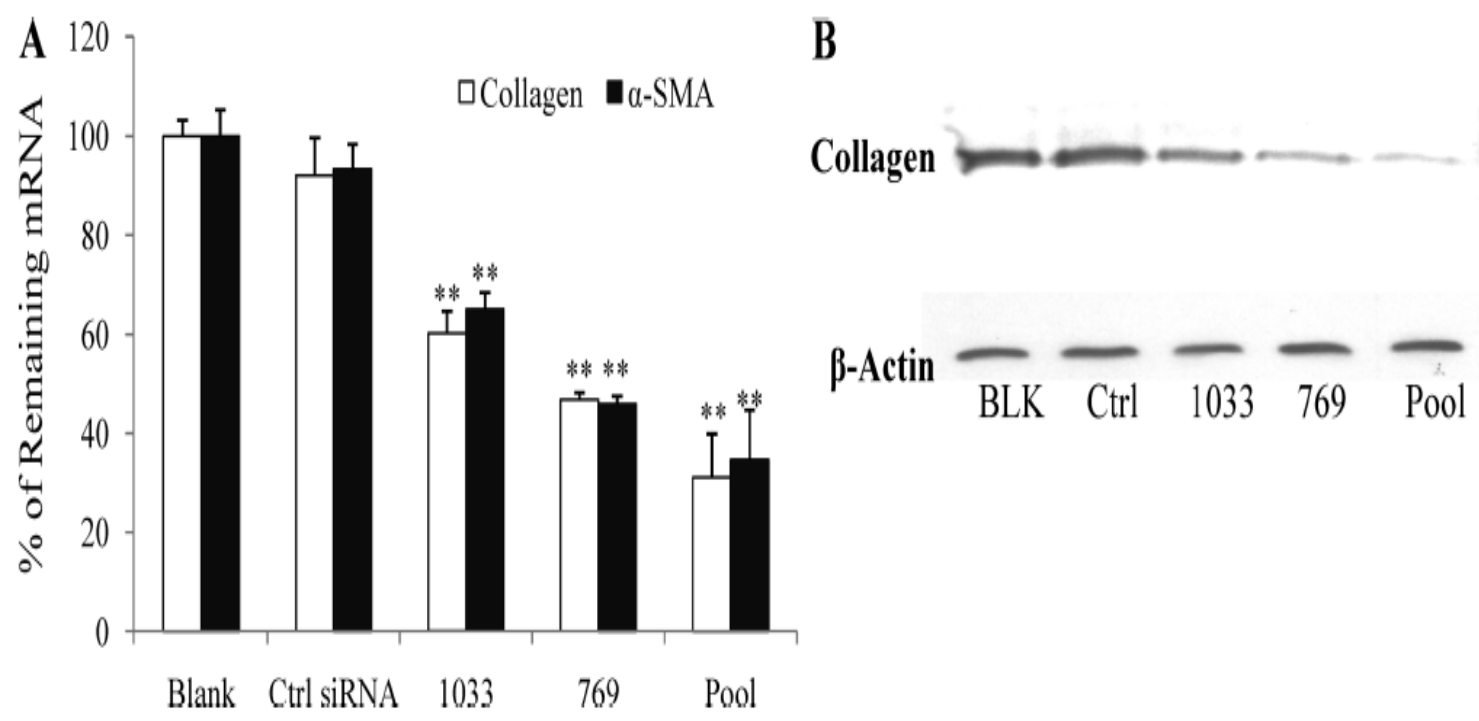

Figure 5-7 Effect of TGF- $\beta 1$ gene silencing on type $\alpha 1$ (I) collagen and $\alpha$-SMA expression after transfection of HSC-T6 cells Lipofectamine 2000/siRNA-1033, 769 and pool complexes. A) At $48 \mathrm{~h}$ post transfection, cells were harvested, total RNA was extracted, and type $\alpha 1$ (I) collagen and $\alpha$-SMA expression was determined at mRNA levels using real time RT-PCR. Results were represented as the mean $\pm \mathrm{SD}(\mathrm{n}=3)$. $*_{*} \mathrm{p}<0.01$. B) Western blot analysis for type $\alpha 1$ (I) collagen expression after TGF- $\beta 1$ gene silencing. 


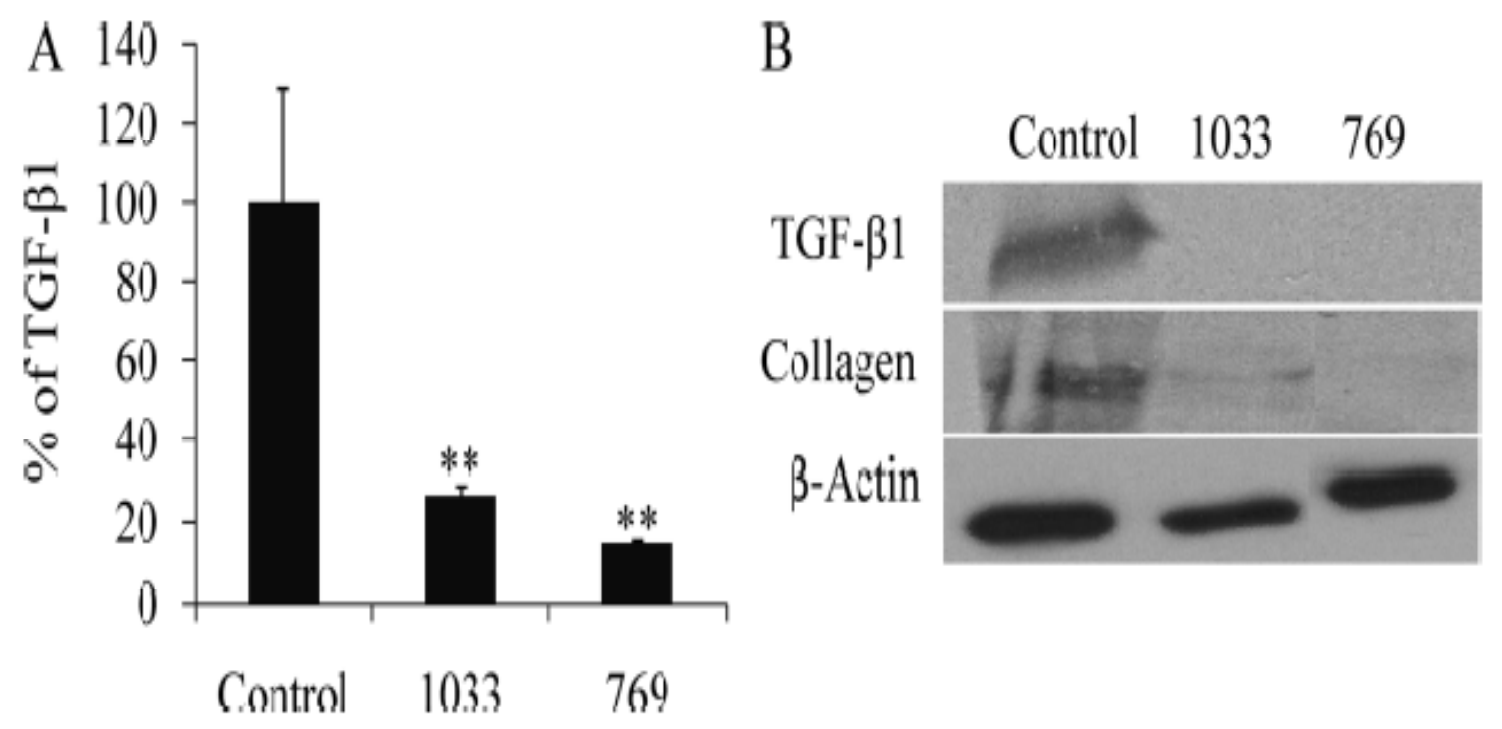

Figure 5-8 Effect of TGF- $\beta 1$ gene silencing on type $\alpha 1$ (I) collagen and TGF- $\beta 1$ expression after transfection of HSC-T6 cells with pshRNA-1033 or pshRNA-769 after complex formation with pyridinium lipid / DOPE cationic liposomes. ${ }^{147}$ At $42 \mathrm{~h}$ post-transfection, cells were harvested, total RNA was extracted, and TGF- $\beta 1$ expression was determined at mRNA levels using real time RT-PCR. A) In addition, protein levels of TGF- $\beta 1$ and type I collagen in the supernatant were determined using Western blot. B) Results were represented as the mean $\pm \mathrm{SD}(\mathrm{n}=3) .{ }^{* *} \mathrm{p}<0.01$. 
significantly higher TGF- $\beta 1$ gene silencing compared to that of the shRNA expression plasmid targeting TGF- $\beta 1$ start site of 769 . There was also similar decrease in TGF- $\beta 1$ gene expression (Figure 5-8B), which is in good agreement with the results of synthetic siRNAs. However, the silencing effect of shRNA was somewhat lower than that of synthetic siRNA targeting at the same coding region.

The shRNA constructed by pSilence1.0, delivered by mixture with Lipo-Lin, shows great transfect effect on protein level. The shRNA expression plasmids targeting TGF- $\beta 1$ start site of 1033 and 769 apparently decreased the expression of both TGF- $\beta 1$ and Collagen type I.

\subsubsection{ELISA Analysis of TNF- $\alpha$ and IL-1 $\beta$}

Since liver inflammation correlates with hepatic fibrosis, silence of TGF- $\beta 1$ might affect inflammation in the liver. To determine whether the down-regulation of TGF- $\beta 1$ decreased the expression of collagen through changing the extent of inflammation, we measured the concentration of TNF- $\alpha$ and IL- $1 \beta$ using ELISA kits.

From Figure 5-9, the amount of TNF- $\alpha$ was decreased to $43.41 \pm 4.03 \%$ and $51.85 \pm 4.75$ respectively comparing to the group which was not treated with TGF- $\beta 1$ shRNA. In the same trend, the level of IL- $1 \beta$ also been down regulated to $49.12 \pm 5.78 \%$ and $59.49 \pm 9.67 \%$ respectively comparing to the control group (Figure 5-9).

\subsubsection{Persistence of Gene Silencing}

To compare siRNA and shRNA on TGF- $\beta 1$ gene silencing, we transfected HSC-T6 cells with siRNA-1033 and pshRNA-TGF- $\beta 1-1033$ after complex formation with pyridinium liposomes at 3/1 (+/-) ratio. As shown in Figure 5-10, the level of TGF- $\beta 1$ mRNA was significantly low for the pshRNA-TGF- $\beta 1$ group, which was lowest at $48 \mathrm{~h}$ post-transfection compared to $24 \mathrm{~h}$ post-transfection for siRNA-TGF- $\beta 1$ group. The level of TGF- $\beta 1$ gene silencing significantly decreased with time, being the lowest at $72 \mathrm{~h}$ post-transfection for siRNA groups. However, for shRNA group, the silence still took effect after $72 \mathrm{~h}$.

\subsection{Discussion}

Liver fibrosis results from chronic injuries to the liver by chronic hepatitis, alcohol abuse, and toxic agents. Among all fibrogenic cytokines, TGF- $\beta$ is the key mediator of liver fibrosis and thus has gained very high attention. TGF- $\beta$ contains three isoforms, such as TGF- $\beta 1$, TGF- $\beta 2$ and TGF- $\beta 3$. TGF- $\beta 1$ is the dominant stimulus to ECM production from HSCs and its expression increases during liver fibrosis. TGF- $\beta 1$ can be secreted by Kupffer cells, biliary cells and infiltrated inflammatory cells, while the autocrine expression of TGF- $\beta 1$ by HSCs is the most important. Compared to quiescent 

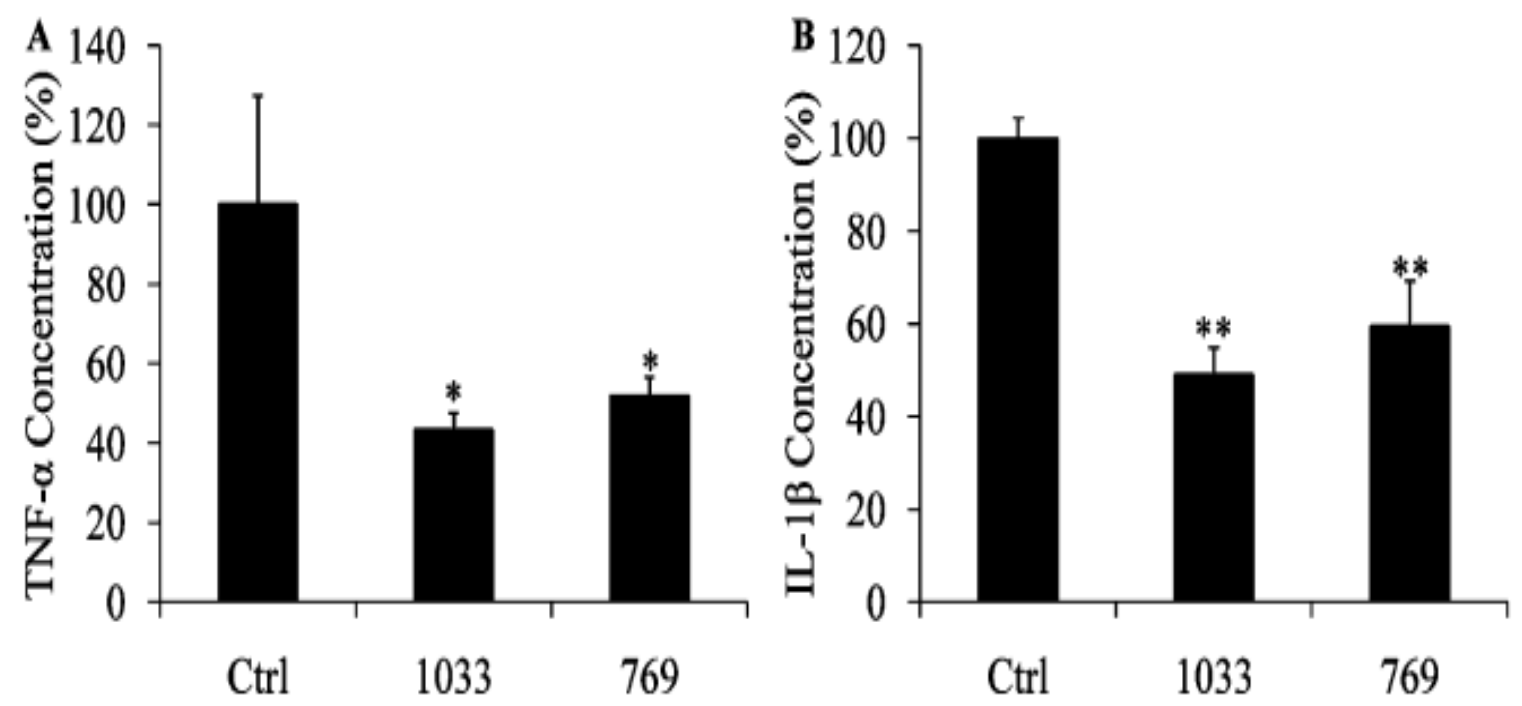

Figure 5-9 Effect of TGF- $\beta 1$ gene silencing on TNF- $\alpha$ and IL- $1 \beta$ secretion. HSC-T6 cells were transfected with pshRNA-1033 and pshRNA-769 after complex formation with pyridinium lipid (C16:1, amide linker, transisomer)/DOPE (1:1 mol/mol) cationic liposomes. Following transfection, TNF- $\alpha$ and IL-1 $\beta$ concentrations were measured by enzyme-linked immunosorbent assay (ELISA). Results were represented as the mean $\pm \mathrm{SD}$ $(\mathrm{n}=3) .{ }^{*} \mathrm{p}<0.05, * * \mathrm{p}<0.01$. 


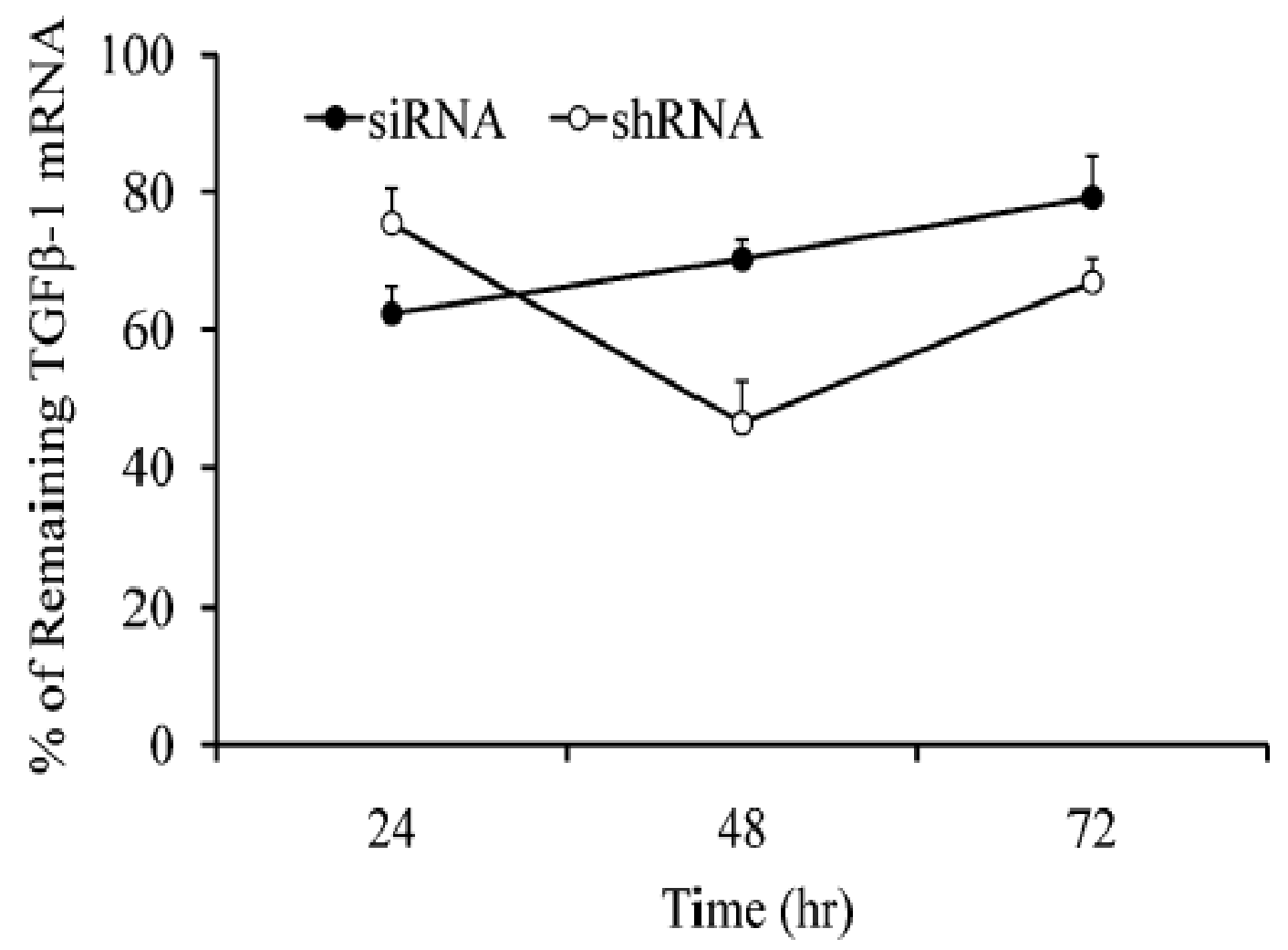

Figure 5-10 Persistence of siRNA and shRNA gene silencing. HSC-T6 cells were transfected with siRNA-1033 and shRNA-769 after complex formation with pyridinium lipid (C16:1, amide linker, trans)/DOPE(1:1 mol/mol) cationic liposomes as described above. After $3 \mathrm{~h}$ of transfection, $200 \mu \mathrm{L}$ of FBS was added and incubated for another 24 $\mathrm{h}, 48 \mathrm{~h}$ and $72 \mathrm{~h}$. At different time points, the cells were collected and total RNA was isolated for real time RT-PCR analysis. 
HSCs, fully activated HSCs are relatively unresponsive to TGF- $\beta 1$, possibly due to the down-regulation of TGF- $\beta$ receptor. TGF- $\beta 1$ knockout mice have shown reduced collagen accumulation to liver injury compared to that of normal mice. ${ }^{252}$ Disrupting TGF- $\beta 1$ synthesis or signaling pathways significantly decreased fibrosis in experimental animal model. ${ }^{237,240}$ However, due to its multiple actions, TGF- $\beta$ represents a potentially important link between neoplasia and fibrosis in the liver. Mutation of TGF- $\beta$ receptor or signaling intermediates has been found in various epithelial cancers. ${ }^{242}$ Strategies aimed at disrupting TGF- $\beta 1$ expression or signaling pathways are being extensively investigated for treating liver fibrosis and various animal studies demonstrated antifibrotic effect. ${ }^{44,237,240-244}$ Currently, neutralizing antibodies, soluble TGF- $\beta$ receptors, antisense oligonucleotide and siRNA were explored to block TGF- $\beta 1$ signaling pathway.

Selection of a potent siRNA sequence targeting a specific gene is a crucial step in developing its therapeutic applications. In this study, we used BLOCK-iT RNAi Designer from Invitrogen to design ten synthetic siRNAs (Table 5-1) targeting at different coding regions of TGF- $\beta 1$ mRNA. As shown in Figure 5-3, only 4 siRNAs showed significant effect on TGF- $\beta 1$ gene silencing, while other 6 siRNAs did not show any effect. All these 4 sRNAs are located in the coding regions of TGF- $\beta 1$ mRNA starting from 769 to 1036. Among these 4 siRNAs, siRNAs targeting 1033 and 1036 coding sites showed the highest TGF- $\beta 1$ gene silencing. This may be due to the local secondary structure of target mRNA.

Two selected siRNAs, such as siRNA-1033 and siRNA-1036, showed dose dependent inhibition of TGF- $\beta 1$ mRNA (Figure 5-3). The silencing effect of siRNA-1036 was similar to that of siRNA-1033 at low concentration, but more significant at high concentration $(150 \mathrm{nM})$. At low concentrations, both siRNAs showed little inhibition and therefore it is difficult to distinguish which one is more effective. Considering there is only 3 base sequence shift between siRNA-1033 and siRNA-1036, the significant difference in silencing indicated that the silencing was very sequence specific.

The in vivo stability of synthetic siRNA and the high cost for in vivo studies hamper its therapeutic application. Therefore, we decided to construct a plasmid based shRNA targeting the most potent region of the TGF- $\beta 1$ mRNA. Compared to synthetic siRNA, shRNA expression plasmid showed relatively poor transfection and gene silencing in HSC-T6 cell lines. The silencing effect of shRNAs targeted at 1033 and 1036 was less than synthetic siRNA targeted at the same position (Figure 5-2 and Figure 5-8A). Nevertheless, shRNA showed similar silencing trend as synthetic siRNA. Its consistent expression ability in the cells and ease to produce makes it a promising vector for therapeutic application.

The development of chronic liver diseases is mediated by sustained hepatic inflammation. Many studies have shown various cytokines, which are crucial indicators of inflammation, such as TGF- $\beta 1$ and TNF- $\alpha$, are very important activators during the process of liver fibrosis. ${ }^{253-255}$ TNF- $\alpha$ has been reported closely related to liver fibrosis and cirrhosis. ${ }^{255}$ We investigated the level of TNF- $\alpha$ after we treated HSCs with TGF- $\beta 1$ shRNA. It was demonstrated that application of TGF- $\beta 1$ shRNA decreased the TNF- $\alpha$ 
concentration in the cell culture serum. The possibility is that decreasing of TGF- $\beta 1$ might down regulate the extent of inflammation and then affect the level of TNF- $\alpha .{ }^{256}$ IL- $1 \beta$ is another very important in the liver fibrosis and enhanced local inflammatory response contribute to IL- 1 beta release, which is crucial to chronic liver fibrosis. IL-1 $\beta$ is another important cytokine, which is known to promote local inflammatory response and consequently promotes chronicliver fibrosis. ${ }^{257}$ Our results also showed that there is significant decrease in secreted IL- $1 \beta$ upon TGF- $\beta 1$ gene silencing (Figure 5-9B). Both TNF- $\alpha$ and IL- $1 \beta$ levels correlated with TGF- $\beta 1$ gene silencing levels.

In summary, we have successfully designed and validated TGF- $\beta 1$-specific siRNAs and then converted two potent siRNA sequences into shRNAs, which effectively silenced TGF- $\beta 1$ gene expression in HSC-T6 cells. TGF- $\beta 1$ gene silencing significantly reduced the production of type I collagen, TIMP-1, and inflammatory cytokines. Our results suggested that silencing of TGF- $\beta 1$ by siRNA and shRNA may be an efficient and more specific approach for therapy of liver fibrosis. 


\section{CHAPTER 6. GFAP PROMOTER-DRIVEN RNA INTERFERENCE ON TGF-B1 TO TREAT LIVER FIBROSIS}

\subsection{Introduction}

Fibrosis is a scarring response that occurs in almost all patients with chronic liver injury. If not controlled, liver fibrosis leads to cirrhosis, which is associated with nodule formation and organ contraction. ${ }^{258}$ Transforming Growth factor beta 1 (TGF- $\beta 1$ ) is a potent stimulus of hepatic stellate cells (HSCs), which produce excess collagen and other extracellular matrices (ECM) ${ }^{259}$ The positive feedback production of TGF- $\beta 1$ by activated HSCs liver injury make situation worse. Therefore, TGF- $\beta 1$ gene silencing produced by HSCs becomes crucial in treating liver fibrosis.

Strategies for disrupting TGF- $\beta 1$ expression are extensively being investigated because inhibiting this cytokine may not only inhibit ECM production but also accelerate its degradation. ${ }^{239}$ The use of different strategies to inhibit TGF- $\beta 1$ gene expression has shown antifibrotic effect for treating liver fibrosis in animal models. ${ }^{44,240-243}$ RNA interference (RNAi) is the phenomenon in which small interfering RNA (siRNA) of 21-23 nt in length silences target gene expression by binding to its complementary mRNA and triggering mRNA degradation. ${ }^{260}$ Compared to antisense ODNs, antibodies and soluble TGF- $\beta 1$ receptor, siRNA is likely to be quite potent in inhibiting TGF- $\beta 1$ gene expression. We have also recently demonstrated siRNA sequence and dose dependent TGF- $\beta 1$ gene silencing. ${ }^{29}$

The standard polymerase III (pol III) promoters, such as U6 and H1, have robust and constitutive activity across multiple cell types. ${ }^{261}$ However, these Pol III promoters do not provide the spatial or temporal control of target gene silencing that is desirable for treating liver fibrosis. TGF- $\beta 1$ is a growth factor involved in many physiological activities. The promoter which can only drive TGF- $\beta 1$ shRNA expression by specific cell types in the body should be utilized for target gene silencing.

In this chapter, to avoid the side effect of nonspecific TGF- $\beta 1$ gene silencing, we constructed an HSC-specific expression plasmid. It is the first time to construct TGF- $\beta 1$ pri-miRNA mimic plasmids driven by glial fibrillary acidic protein (GFAP) promoter, which relies on RNA polymerase II to achieve RNAi if the shRNA inserts are bracketed by pri-miRNA backbone. Even though we found that the silencing level of GFAP driven TGF- $\beta 1$ pri-miRNA mimics were less than U6 drivien ones, the pri-miRNA cluster mimics of two shRNA inserts showed high silencing effect to compensate this phenomenon. 


\subsection{Materials and Methods}

\subsubsection{Materials}

Dulbecco's modified Eagle's medium (DMEM), penicillin G (5000 U/mL), trypsin-EDTA, Trizol, DNase I, and Lipofectamine 2000 were purchased from Invitrogen (Carlsbad, CA). pSilencer1.0 was purchased from GenScript Corporation (Piscataway, NJ). All of the restriction enzymes were purchased from New England Biolabs (Ipswich, MA). SYBR Green-1 dye universal master mix and MultiScribe reverse transcriptase were purchased from Applied Biosystems, Inc. (Foster City, CA). All the primers for Real Time PCR were purchased from Integrated DNA Technologies, InC. TGF- $\beta 1$ primary antibody, type $\alpha 1$ (I) collagen primary antibody and rabbit anti-rat secondary antibody were purchased from Santa Cruz Biotech, Inc. (Santa Cruz, CA). TNF- $\alpha$ enzyme-linked immunosorbent assay (ELISA) kits were purchased from eBioscience, Inc. (San Diego, CA).

\subsubsection{Construction of shRNA Expression Plasmids}

Based on our recently published results, we selected two potent siRNA sequences targeting 769 and 1033 start sites of TGF- $\beta 1$ mRNA and converted them into shRNA sequences for cloning into pSilencer 1.0 vector, which carries an shRNA expression cassette under the control of a U6 promoter. These shRNAs contain two complementary oligonucleotides, which were annealed to form a double stranded DNA for ligation into pSilencer vector. pU6-shRNA-769, pU6-shRNA-1033, pU6-shRNA-769+1033 and pU6-shRNA-scramble were constructed as mentioned in our previous study. ${ }^{29}$ For HSC-specific gene silencing, GFAP promoter was cloned by the Long Range PCR kit by using pGFA2 vector as a template, which is a gift from Dr. Michael Brenner of the Depatment of Neurology at the University of Alabama. Then, GFAP promoter fragment was inserted into pRNAT H1.1 to replace the $\mathrm{H} 1$ promoter in pRNAT H1.1 to make pGFA-RNAT H1.1 vector. pGFAP-mi-shRNAs were constructed by inserting the pre-designed mi-shRNA sequence with microRNA 30 context to pGFA-RNAT H1.1. The sequences for all the predesigned mi-shRNA sequences are shown in Table 6-1.

\subsubsection{Transfection}

The day before transfection, HSC-T6 cells were seeded in 6 well plate at a number of $1 \times 10^{6}$ cells per well. When the cell confluence reached 80 to $90 \%$, cells were transfected with shRNA expression vectors at doses of $1 \mu \mathrm{g} / \mathrm{well}$. The shRNA plasmids were mixed with Lipofectamine 2000 at a 1:3 w/v $(\mu \mathrm{g} / \mu \mathrm{L})$ ratio in $250 \mu \mathrm{L}$ Dulbecco's Modified Eagle's Medium (DMEM) without serum for $20 \mathrm{~min}$ at room temperature to allow complex formation. The transfection mixture was then added to each plate with $2 \mathrm{~mL}$ fetal bovine serum (FBS) free DMEM. After $4 \mathrm{~h}$ of incubation, $200 \mu \mathrm{L}$ FBS was added per well and incubated for additional $42 \mathrm{~h}$. 
Table 6-1 Pri-miRNA mimic inserts sequences.

\begin{tabular}{|c|c|}
\hline mi-shRNA insert & Sequence \\
\hline mi-shRNA-1033 & $\begin{array}{l}\text { GGATCCGTCGACTAGGGATAACAGGGTAATTGTTTGAA } \\
\text { TGAGGCTTCAGTACTTTACAGAATCGTTGCCTGCACAT } \\
\text { CTTGGAAACAGCTGGGATTACTTCTTCAGGTTAACCCA } \\
\text { ACAGAAGGCTCGAGAAGGTATATTGCTGTTGACAGTG } \\
\text { AGCGCCGCAGCTGTACATTGACTTTGTGAAGCCACAGA } \\
\text { TGTAAAAGTCAATGTACAGCTGCTGCCTACTGCCTCGT } \\
\text { CTAGAAAGGGGCTACTTTAGGAGCAATTATCTTGTTTA } \\
\text { CTAAAACTGAATACCTTGCTATCTCTTTGATACATTTTT } \\
\text { TGGATCC }\end{array}$ \\
\hline mi-shRNA-769 & $\begin{array}{l}\text { GGATCCGTCGACTAGGGATAACAGGGTAATTGTTTGAA } \\
\text { TGAGGCTTCAGTACTTTACAGAATCGTTGCCTGCACAT } \\
\text { CTTGGAAACAGCTGGGATTACTTCTTCAGGTTAACCCA } \\
\text { ACAGAAGGCTCGAGAAGGTATATTGCTGTTGACAGTG } \\
\text { AGCGCCGAACCAAGGAGACGGAATAGTGAAGCCACAG } \\
\text { ATGTATATTCCGTCTCCTTGGTTCTGCCTACTGCCTCGT } \\
\text { CTAGAAAGGGGCTACTTTAGGAGCAATTATCTTGTTTA } \\
\text { CTAAAACTGAATACCTTGCTATCTCTTTGATACATTTTT } \\
\text { TGGATCC }\end{array}$ \\
\hline mi-shRNA-769+1033 & $\begin{array}{l}\text { GGATCCGTCGACTAGGGATAACAGGGTAATTGTTTGAA } \\
\text { TGAGGCTTCAGTACTTTACAGAATCGTTGCAGCTGTAC } \\
\text { ATTGACTTTGTGAAGCCACAGATGTAAAAGTCAATGTA } \\
\text { CAGCTGCGCCTGCACATCTTGGAAACAGCTGGGATTAC } \\
\text { TTCTTCAGGTTAACCCAACAGAAGGCTCGAGAAGGTAT } \\
\text { ATTGCTGTTGACAGTGAGCGCCGAACCAAGGAGACGG } \\
\text { AATAGTGAAGCCACAGATGTATATTCCGTCTCCTTGGT } \\
\text { TCTGCCTACTGCCTCGTCTAGAAAGGGGCTACTTTAGG } \\
\text { AGCAATTATCTTGTTTACTAAAACTGAATACCTTGCTAT } \\
\text { CTCTTTGATACATTTTTGGATCC }\end{array}$ \\
\hline
\end{tabular}




\subsubsection{Real Time PCR}

Following transfection, total RNA was extracted from the cells by RNAeasy Mini Kit and the RNA concentration was measured by UV spectrophotometry using Nanodrop spectronanometer (Thermo Scientific, Pittsburgh, PA). Then, 385 ng of the total RNA per sample was reverse transcribed into cDNA using MultiScribe Reverse Transcriptase Reagent and random hexamers. The obtained cDNA was amplified by real time PCR using SYBR Green-1 dye universal master mix on ABI Prism 7700 Sequence Detection System (Applied Biosystems, Inc., Foster City, CA).

\subsubsection{Western Blot Assay}

Transfected cells were lysed using $1 \times$ Laemmli sodium dodecyl sulfate (SDS) sample buffer. The lysed protein was then boiled at $100^{\circ} \mathrm{C}$ for $5 \mathrm{~min}$ and subjected to $4 \%$ or $10 \%$ SDS-polyacrylamide (SDS-PAGE) gel electrophoresis and subsequent transfer by iBLot system. After blocking with 5\% nonfat dried milk in $1 \times$ PBST containing $0.05 \%$ Tween-20 in PBS for $1 \mathrm{~h}$ at room temperature, the membranes were incubated with goat anti-rat type I collagen, rabbit anti-rat TGF- $\beta 1$ and goat anti-rat $\beta$-actin primary antibodies for $16 \mathrm{~h}$ at $4^{\circ} \mathrm{C}$. Membrane was then incubated with horseradish peroxidase conjugated goat anti-rabbit secondary antibody for $1 \mathrm{~h}$ at room temperature. Target proteins were detected by enhanced chemiluminescence detection kit (ECL, GE Healthcare).

\subsubsection{Trypan Blue Staining}

Transfected HSC-T6 cells were suspended in DMEM medium at a concentration of $1 \times 10^{5} / \mathrm{mL}$ in a $1.5 \mathrm{~mL}$ tube. Five hundred microliter of cells and $0.1 \mathrm{~mL} 0.4 \%$ trypan blue staining buffer were mixed thoroughly. The staining samples were allowed standing at the room temperature for $5 \mathrm{~min}$ and then $10 \mu \mathrm{L}$ was applied to a hemocytometer for cell counting.

\subsubsection{ELISA Assay of TNF- $\alpha$}

After transfection, the supernatant of the cultured cells was collected and the concentration of TNF- $\alpha$ was measured using enzyme-linked immunosorbent assay (ELISA) (eBioscience, San Diego, CA), according to the manufacturer's protocol.

\subsubsection{HSC Wound Healing Assay}

Wound healing assay of transfected HSC-T6 cells was carried out as described by Liu et al. ${ }^{262}$ with minor modification. Briefly, HSC-T6 cells were seeded in 6-well plates at a number of $1 \times 10^{6}$ per well and allowed to grow in DMEM growth medium containing 
$10 \%$ serum. The cells were then deprived of serum for $6 \mathrm{~h}$ when the cell's confluence reached 95\%. Then, the cells were transfected with Lipofectamine 2000/pshRNA complexes at a dose of $1 \mu \mathrm{g}$ plasmid per well. After transfection, the cells were still cultured in DMEM without serum. The cell monolayer was disrupted by a scratch to mimic wound. The cells were then cultured for additional $16 \mathrm{~h}$, and the wound was observed under a microscope.

\subsubsection{Cell Migration Assay}

Migration assays were performed in Transwell membrane filter inserts in 24-well tissue culture plates (BD Labware, Bedford, MA) with the pore size in polycarbonate membranes of $8 \mu \mathrm{m}$. The lower surface of inserts membranes were pre-incubated with fibronectin diluted in $10 \mathrm{mmol} / \mathrm{L} \mathrm{NaHCO}_{3} 4^{\circ} \mathrm{C}$ overnight and then blocked with $0.1 \%$ heat-inactivated BSA at $37^{\circ} \mathrm{C}$ for $30 \mathrm{~min}$. Cells tansfected with pri-miRNA mimics were detached, washed once in PBS, and then resuspended in DMEM containing $0.1 \%$ BSA. A $300 \mu \mathrm{L}$ cell suspension containing $8 \times 10^{3}$ cells was added to each insert. DMEM containing $1 \%$ FBS was added to the lower wells in the 24 -well cell culture plate. Migration was allowed to proceed at $37^{\circ} \mathrm{C}$ for $4 \mathrm{~h}$. Cells that did not migrate through the filters were removed by cotton swabs. After fixing and staining with Diff-Quick (Baxter Healthcare Corp., McGraw Park, IL), the cells were photographed by microscope.

\subsection{Results}

\subsubsection{Effect of shRNA Sequence on TGF- $\beta 1$ Silencing}

To examine the effect of shRNA sequence on TGF- $\beta 1$ gene silencing, we transfected HSC-T6 cells with pU6-shRNA-scramble, pU6-shRNA-769, pU6-shRNA-1033 and pU6-shRNA-769+1033 respectively after complex formation with Lipofectamine 2000. Real time RT-PCR results (Figure 6-1A) showed decrease in TGF- $\beta 1$ gene expression in HSC-T6 cells to $53.60 \pm 1.33 \%, 48.05 \pm 7.51 \%$ and $24.09 \pm 0.25 \%$ by pU6-shRNA-769, pU6-shRNA-1033 and pU6-shRNA-769+1033, respectively, compared to pU6-shRNA-scramble. The plasmid encoding two shRNAs targeting two different regions such as 769 and 1033 start sites of TGF- $\beta 1$ mRNA were more effective in TGF- $\beta 1$ gene silencing, compared to the plasmids encoding single siRNA.

TGF- $\beta 1$ gene silencing should enhance the degradation of type I collagen. We, therefore, determined the effect of TGF- $\beta 1$ gene silencing on type I collagen protein expression by Western blot analysis. There was significant decrease in collagen of pU6-shRNA-769, pU6-shRNA-1033 and pU6-shRNA-769+1033 treated groups, while little decrease in collagen concentration for the control shRNA treated samples (Figure 6-1B). 


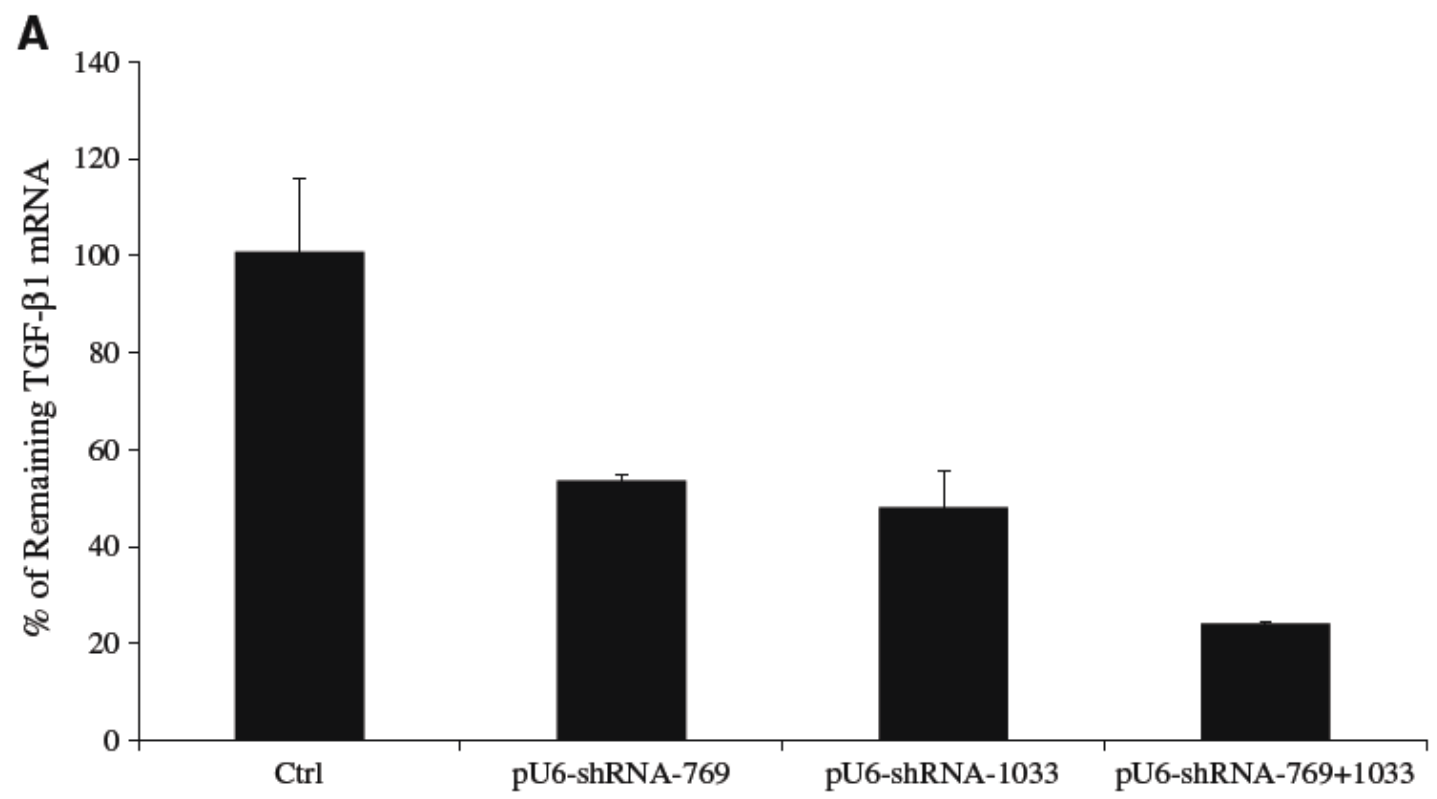

B

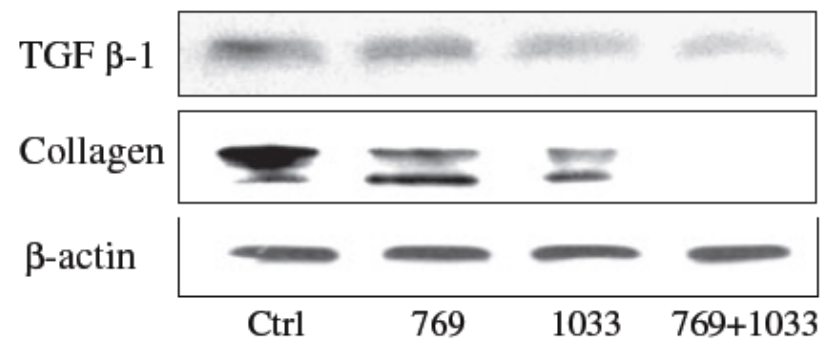

Figure 6-1 Effect of shRNA sequences on TGF- $\beta 1$ gene silencing and its effect on collagen gene expression. HSC-T6 cells were transfected by pU6-shRNA-scramble, pU6-shRNA-769, pU6-shRNA-1033 and pU6-shRNA-769+1033, respectively after complex formation with Lipofectamine 2000. A) Real-time RT-PCR. Results are expressed as the mean $\pm \mathrm{SD}(\mathrm{n}=4)$. B) Western blot analysis. 


\subsubsection{Effect of Promoters and Pri-miRNA Mimics on TGF- $\beta 1$ Gene Silencing}

For HSC-specific gene silencing, we used pGFA-shRNA-1033 driven by GFAP promoter but failed to produce any TGF- $\beta 1$ silencing effects (Figure 6-2A). However, still directed by GFAP promoter, the pri-miRNA mimic, pGFA-mi-shRNA-1033, reduced the total amount of TGF- $\beta 1$ mRNA to $44.05 \pm 1.60 \%$ of that in the HSC-T6 cell line administrated by pGFA-RNAT H1.1 with scramble sequence as the control (Figure 6-2A). pGFA-mi-shRNA-1033 driven by GFAP promoter had less silencing efficiency than pU6-shRNA-1033 driven by U6 promoter by comparing the third bar and the fourth bar in Figure 6-2A. The total amount of TGF- $\beta 1$ mRNA in HSC-T6 treated by pU6-shRNA was only $29.28 \pm 1.59 \%$ of the control.

HSC-T6 cells were transfected by GFAP promoter driven plasmids, which produced pri-miRNA mimics or pri-miRNA cluster mimics and decreased TGF- $\beta 1$ mRNA level (Figure 6-2B). Real time RT-PCR results revealed TGF- $\beta 1$ gene expression in HSC-T6 cells was decreased to $69.54 \pm 5.04 \%, 45.95 \pm 2.45 \%$ and $35.65 \pm 3.82 \%$ by pGFA-mi-shRNA-769, pGFA-mi-shRNA-1033 and pGFA-mi-shRNA-769+1033, respectively, compared to the control.

\subsubsection{HSC-T6 Proliferation and Apoptosis}

To determine the effect of GFAP driven TGF- $\beta 1$ pri-miRNA mimics and pri-miRNA cluster mimics on HSC-T6 proliferation and apoptosis, we did MTT assay. pGFA-mi-shRNA-769, pGFA-mi-shRNA-1033 and pGFA-mi-shRNA-769+1033 effectively prevented HSC-T6 cell proliferation (Figure 6-3A). Similarly, Western blot analysis showed decrease in the phosphorylation of extracellular-signal related kinase (p-ERK) due to TGF- $\beta 1$ gene silencing, which further proved the inhibition of HSC-T6 proliferation after transfection of HSC-T6 cells with TGF- $\beta 1$ pGFA-mi-shRNA plasmids (Figure 6-3B). Western blot of ERK did not show any change in band thickness, suggesting an equal amount of proteins in each sample.

Trypan blue staining and caspase activity analysis demonstrateded that TGF- $\beta 1$ silencing also increased HSC-T6 apoptosis (Figure 6-4A, B). The caspase-3/7 activity was dramatically increased in HSC-T6 cells after transfection of HSC-T6 cells with pGFA-mi-shRNAs, especially for 769+1033 cluster (Figure 6-4A), which correlated well with decrease in HSC-T6 cell viability (Figure 6-4B).

\subsubsection{TGF- $\beta 1$ pGFA-mi-shRNAs Decreases TNF- $\alpha$ Amount in Cell Culture Medium}

TNF- $\alpha$ concentration was decreased after transfection with pGFA-mi-shRNA

plasmids. pGFA-mi-shRNA-769, pGFA-mi-shRNA-1033 and pGFA-mi-shRNA-769+1033 decreased TNF- $\alpha$ concentration in HSC-T6 cell culture medium to $281.27,275.85$ and $235.55 \mathrm{pg} / \mathrm{mL}$ from $483.50 \mathrm{pg} / \mathrm{mL}$ of the control group (Figure 6-5). 

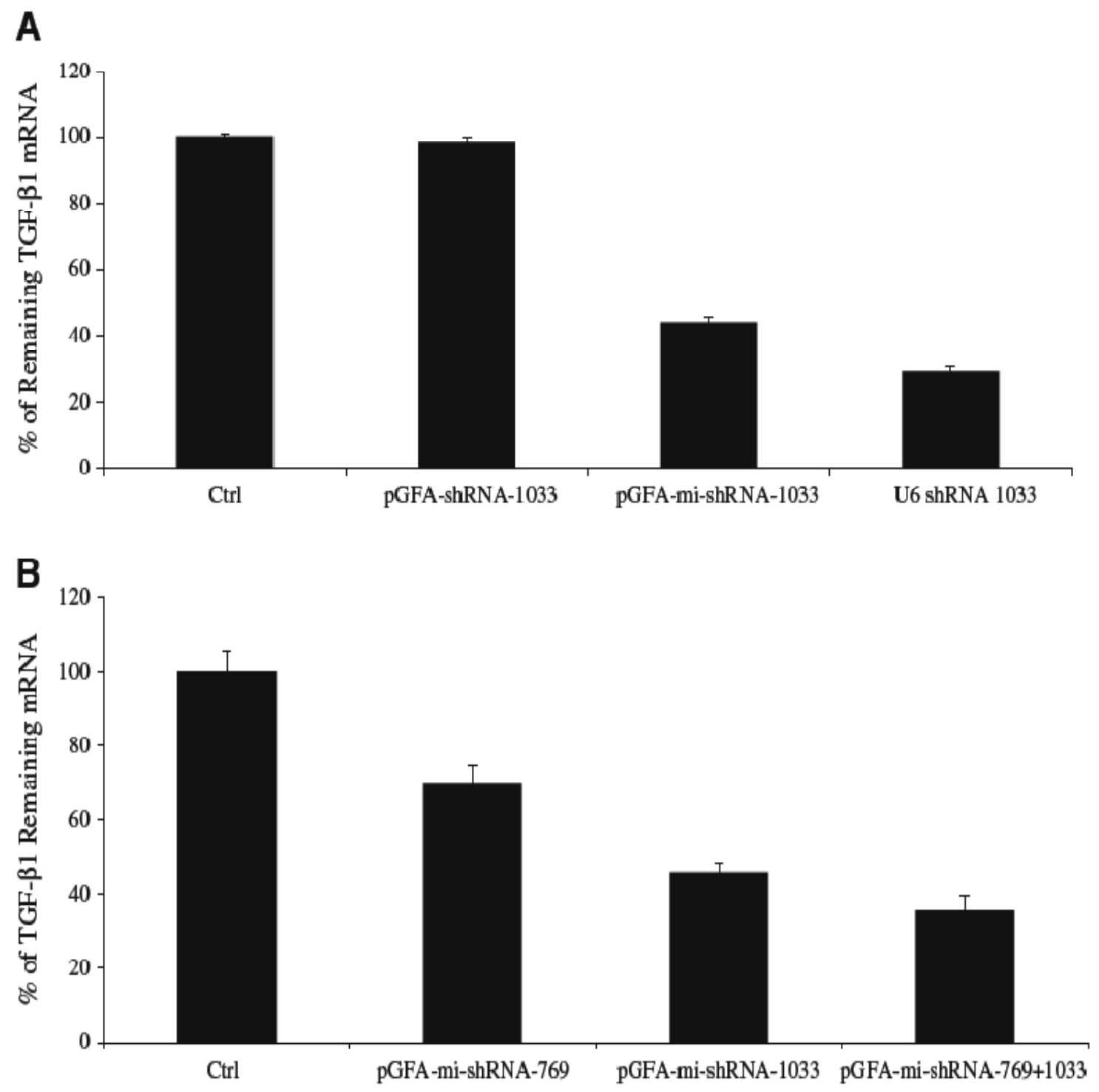

Figure 6-2 Effects of promoters and pri-miRNA mimics on TGF- $\beta 1$ silencing. After $42 \mathrm{~h}$ post-transfection of pri-miRNA mimics and pri-miRNA cluster mimics, cells were harvested, total RNA was extracted, and TGF- $\beta 1$ gene expression was determined at mRNA levels using real-time RT-PCR. Results were represented as the mean $\pm \mathrm{SD}(\mathrm{n}=3)$. A) pGFA-shRNA-1033 showed no TGF- $\beta 1$ gene silencing, whereas pGFA-mi-shRNA-1033 reduced the total amount of TGF- $\beta 1$ mRNA. pGFA-mi-shRNA-1033 had less silencing efficiency than pU6-shRNA-1033. B) pGFA-mi-shRNA-769, pGFA-mishRNA-1033 and pGFA-mishRNA-769+1033 decreased TGF- $\beta 1$ mRNA level in HSC-T6 cells. pGFA-mi-shRNA-769+1033, producing pri-miRNA cluster mimics, had more silencing effects. 


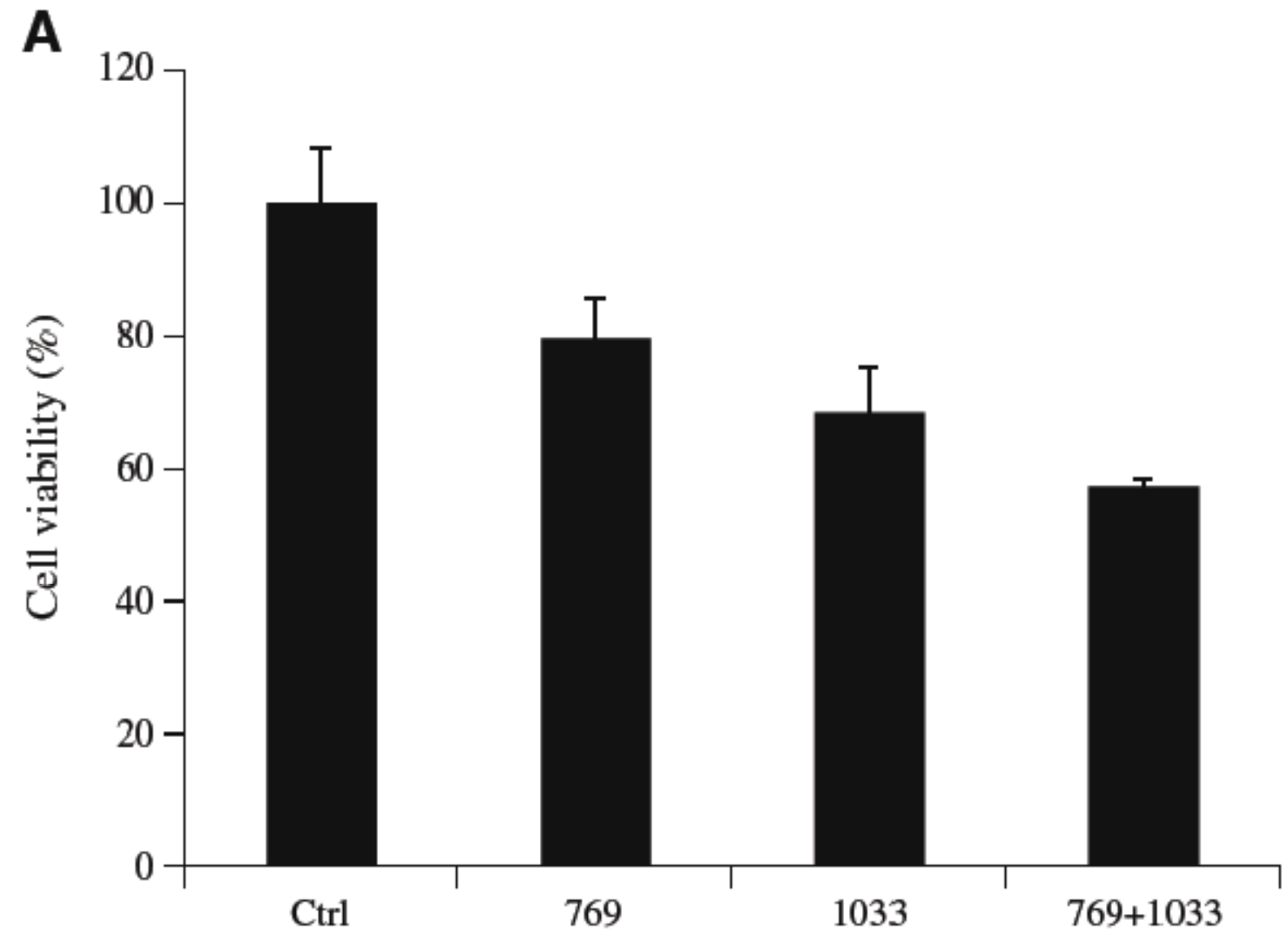

B

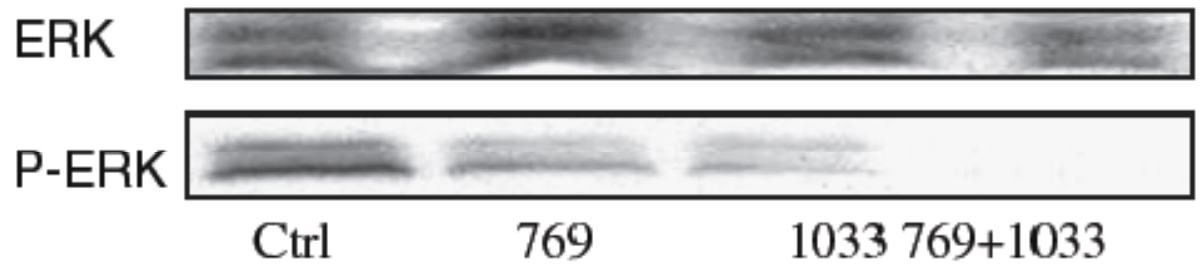

Figure 6-3 Effects of GFAP-driven TGF- $\beta 1$ pri-miRNA mimics and pri-miRNA cluster mimics on HSC-T6 proliferation. After $42 \mathrm{~h}$ posttransfection with TGF- $\beta 1$ pri-miRNA mimics plasmids and pri-miRNA cluster mimics plasmids, cells were harvested. A) MTT assay showed the cell viability of the HSC-T6 cells transfected by pGFA-control, pGFA-mi-shRNA-769, pGFAmi-shRNA-1033 and pGFA-mi-shRNA-769+1033. Results are expressed as the mean $\pm \mathrm{SD}(\mathrm{n}=3)$. B) Western blot analysis of $\mathrm{p}$-ERK after transfection of HSC-T6 cells with pGFA-control, pGFA-mishRNA-769, pGFA-mi-shRNA-1033 and pGFA-mi-shRNA-769+1033. Western blot analysis of ERK was also performed for normalizing the amounts of proteins in each sample. 

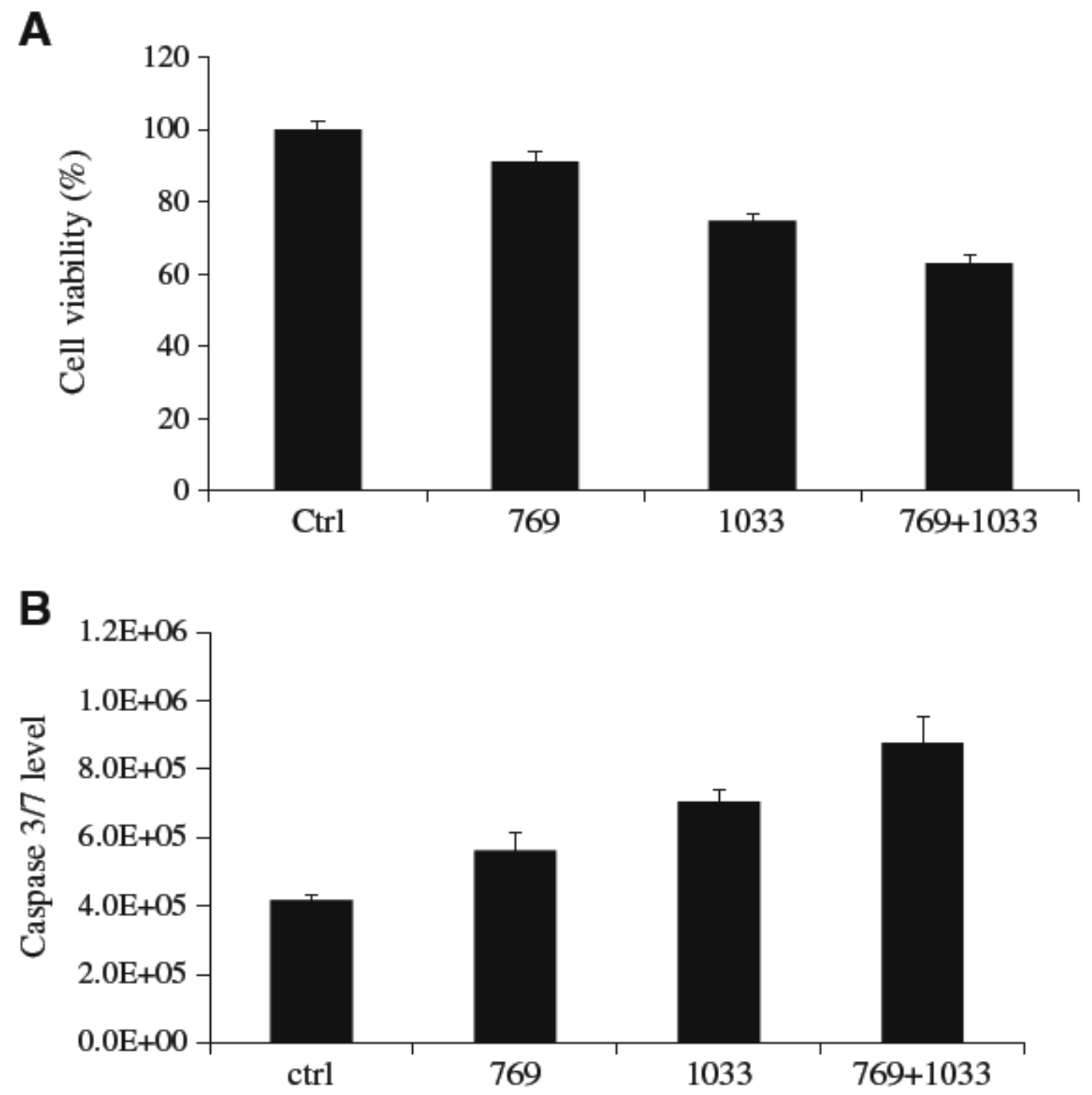

Figure 6-4 Effects of GFAP-driven TGF- $\beta 1$ pri-miRNA mimics and pri-miRNA cluster mimics on HSC-T6 apoptosis. After $42 \mathrm{~h}$ post-transfection with TGF- $\beta 1$ pri-miRNA mimics plasmids and pri-miRNA cluster mimics plasmids, cells were harvested. A) caspase activity analysis demonstrated the change after transfection of HSCT6 cells with pGFA-control, pGFA-mi-shRNA-769, pGFA-mi-shRNA-1033 and pGFA-mi-shRNA-769+1033. B) trypan blue staining displayed the ratio of apoptotic HSC-T6 cells after transfection with pGFA-control, pGFA-mi-shRNA-769, pGFA-mi-shRNA-1033 and pGFA-mi-shRNA-769+1033. Results are expressed as the mean $\pm \mathrm{SD}(\mathrm{n}=4)$. 


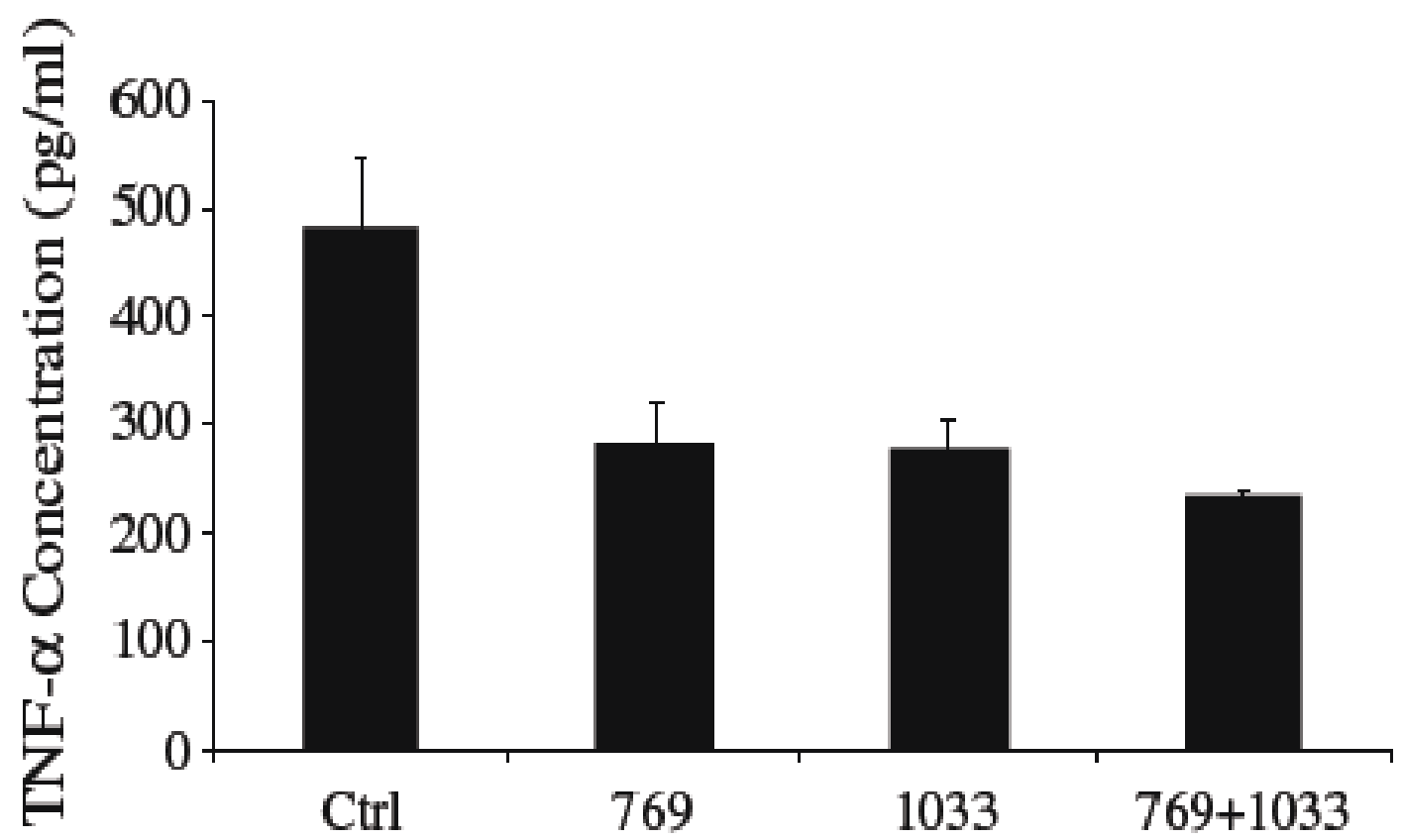

Figure 6-5 Effects of GFAP-driven TGF- $\beta 1$ pri-miRNA mimics and pri-miRNA cluster mimics on secretion of inflammatory cytokines by HSC-T6 cells. After $42 \mathrm{~h}$ post-transfection with TGF- $\beta 1$ pri-miRNA mimics plasmids and primiRNA cluster mimics plasmids, concentration of TNF- $\alpha$ in cell culture medium was measured by ELISA. Results are expressed as the mean $\pm \mathrm{SD}(\mathrm{n}=4)$. 


\subsubsection{Pmi-shRNAs-TGF- $\beta 1$ Impedes HSC-T6 Migration}

The wounded HSC-T6 monolayers transfected with GFAP promoter driven scrambled shRNA expression vectors induced cell migration leading to wound closure at $16 \mathrm{~h}$ after wounding (Figure 6-6A.a). However, transfection of pGFA-mi-shRNA-769 (Figure 6-6A.b), pGFA-mi-shRNA-1033 (Figure 6-6A.c) and pGFA-mi-shRNA-769+1033 (Figure 6-6A.d) impeded wound closure and remained wound gaps. In the directional cell migration toward fetal bovine sera, HSC-T6 transfected with pGFA-mi-shRNA-769+1033 showed the least migration ability (Figure 6-6B.d) compared to the control group (Figure 6-6B.a). Both of groups transfected with pGFA-mi-shRNA-769 (Figure 6-6B.b), pGFA-mi-shRNA-1033 (Figure 6-6B.c) also showed less migration.

\subsubsection{Cell Specificity of GFAP Promoter Driven shRNA Expression}

After transfection with pGFA-mi-shRNA-1033 and U6-mi-siRNA-1033, the remaining TGF- $\beta 1 \mathrm{mRNA}$ level in rat INS-1E cell line was decreased to $57.77 \pm 7.55 \%$ by U6 promoter driven plasmids, compared to the blank control (Figure 6-7). However, GFAP promoter did not show this phenomenon.

\subsection{Discussion}

Chronic liver injuries often activate HSCs to overproduce ECM proteins and several fibrogenic proteins. Among them, TGF- $\beta 1$ is the key mediator of liver fibrosis. TGF- $\beta 1$ knockout mice have shown reduced collagen accumulation in response to liver injury. ${ }^{252}$ Inhibition of TGF- $\beta 1$ gene expression or its signaling has been shown to decrease fibrosis in experimental animal model. ${ }^{251,263}$ Therefore, in this study, we selected two potent siRNA sequences for converting into shRNA and then cloning into pSilencer1.0 vector driven by a ubiquitous $\mathrm{U} 6$ promoter due to its relatively ease of producing shRNA transcripts. Transfection of HSC-T6 cells with these plasmids showed decent down-regulation of TGF- $\beta 1$ mRNA. Co-expression of two shRNAs targeting 769 and 1033 TGF- $\beta 1$ mRNA start sites showed higher TGF- $\beta 1$ gene silencing. This finding is in good agreement with our previous study where we demonstrated that the mixture of two potent siRNA sequences, targeting 769 and 1033 start sites of TGF- $\beta 1$ mRNA showed higher TGF- $\beta 1$ gene silencing compared to single siRNA application at the same total concentration. $^{29}$

TGF- $\beta 1$ is overexpressed by HSCs and U6 promoter drives high levels of shRNA expression by all types cells which may elicit toxicity. Pri-miRNA mimics have the potential to mitigate this problem as they can utilize low expression RNA polymerase II (pol II) promoter. GFAP is an intermediate filament protein identified as a biomarker for both quiescent and activated HSCs. ${ }^{264}$ Furthermore, GFAP promoter has recently been utilized for HSC-specific gene expression ${ }^{265,266}$ and is effective controlled by pol II, which can only recognize microRNA but not shRNA. Therefore, we selected miR-30 


\section{A}
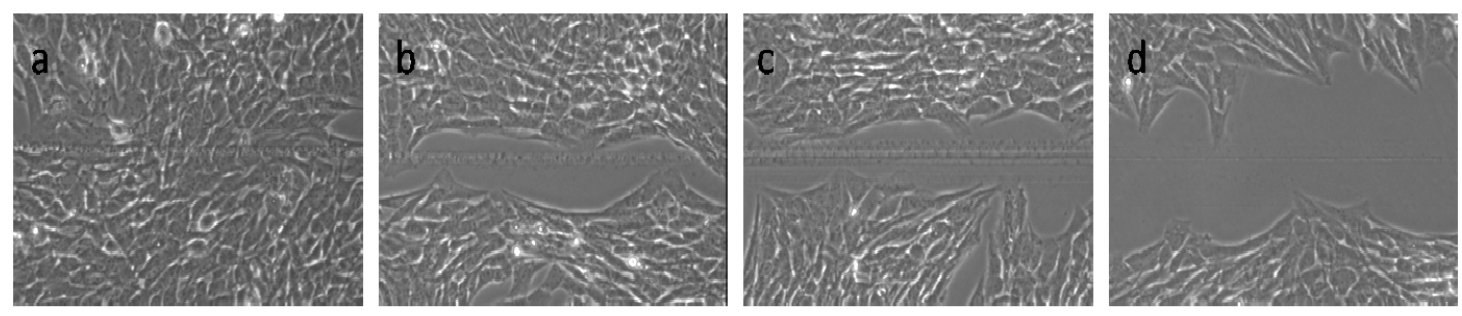

B
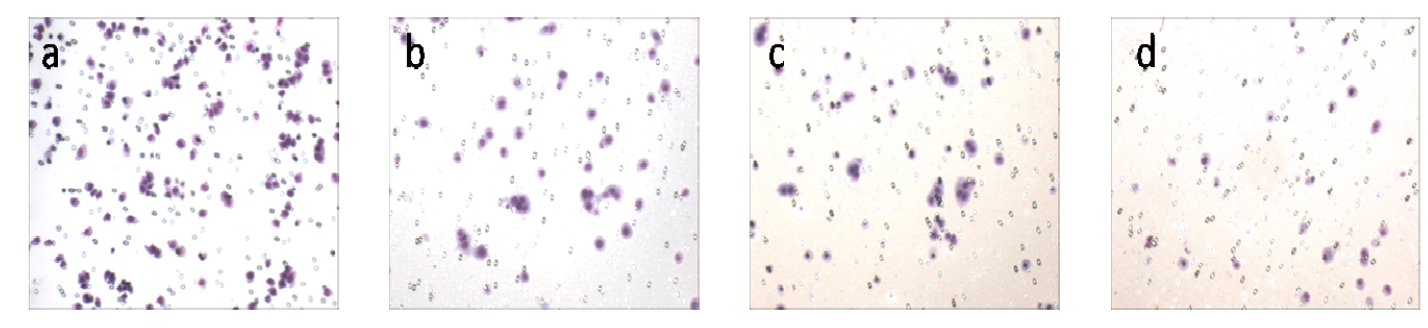

Figure 6-6 Wound-healing and chamber migration assays. A) HSCs were grown to confluence and were disrupted to generate a linear wound, and then transfected with a) pGFA-control, b) pGFA-mi-shRNA-769, c) pGFAmi-shRNA-1033 and d) pGFA-mi-shRNA-769+1033 plasmids after complex formation with lipofectamine 2000 at a dose of $1 \mu \mathrm{g} /$ well. After another $16 \mathrm{~h}$ incubation in none serum medium, photographs were taken by microscope. B) After transfection with a) pGFA-control, b) pGFA-mishRNA-769, c) pGFA-mi-shRNA-1033, and d) pGFA-mi-shRNA-769+1033 plasmids, purple spots indicated the migrated HSC-T6 cells to the bottom of the chambers. 


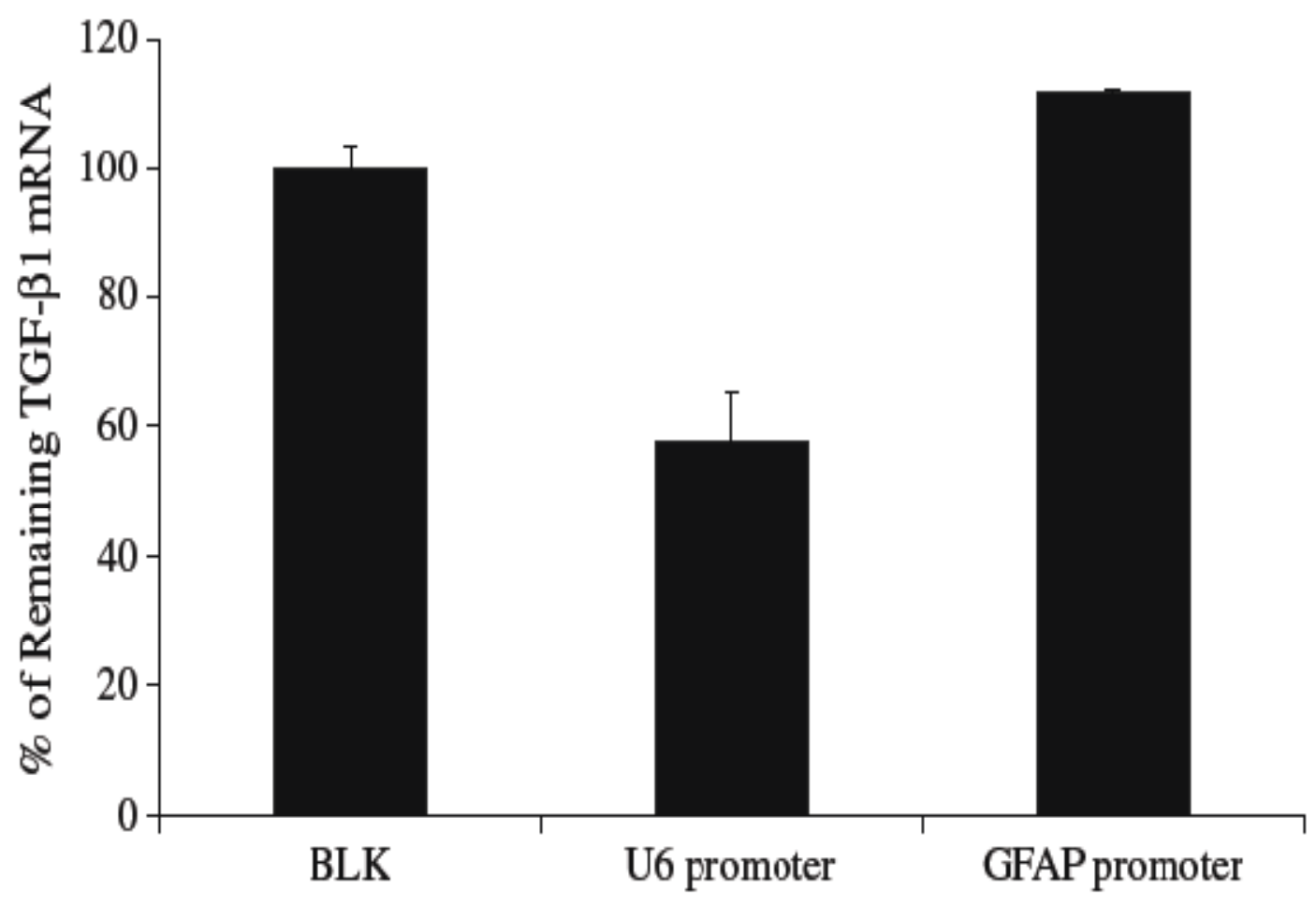

Figure 6-7 Cell specificity of GFAP promoter-driven shRNA expression. After transfection with pGFA-mi-shRNA-1033 and U6-mi-siRNA-1033, the remaining TGF- $\beta 1$ mRNA level in rat INS-1E cell line was decreased to $57.77 \pm 7.55 \%$ by U6 promoter driven plasmids, compared to the blank control. 
to be incorporated into the constructed plasmids. miR-30 has been investigated a lot in recent years, which are involved in many areas in the body. miR-30 was upregulated in activated HSCs. ${ }^{267} \mathrm{Ji}$ et al. demonstrated the expression of miRNA-30 was upregulated to more than 3 folds in activated HSCs than quiescent HSCs. This phenomenon facilitates TGF- $\beta 1$ gene silencing in activated HSCs and reduces the unnecessary silencing in quiescent HSCs. However, there were some studies already demonstrated that the silencing effects of pri-miRNA mimics were decreased compared to matched shRNAs. ${ }^{268,269}$ It is good for the body because there may be some protective machinery from excessive intrinsic microRNA production to prevent toxicity in the cells, compared to artificial shRNA. In our study, the pri-miRNA mimics driven by GFAP promoter showed TGF- $\beta 1$ gene silencing significantly less than the matched shRNA driven by U6 promoter (Figure 6-2A). The choice between target expression and non-specific expression was affected by the balance between shRNAs and pri-miRNA mimics silencing effects. Fortunately, the pri-miRNA cluster mimics, which can target multiple sites in the same gene, in our case is TGF- $\beta 1$, controlled TGF- $\beta 1$ level to a lower level relative to pri-miRNA mimics (Figure 6-2B).

Besides the function of promoters, the secondary structure of pre-miRNA mimics plays an important role in efficient processing of miRNA-based silencing in vitro ${ }^{270}$ and in vivo. ${ }^{271}$ The extended stem-loop structure in pri-miRNA mimics need to be bracketed by single stranded RNA, otherwise Drosha cannot recognize pri-miRNA transcriptions and process them correctly. ${ }^{272}$ From the secondary RNA structure software RNAstructure version 5.1, the pre-miRNA cluster mimic designed by our lab did have its extended stem-loop structure been flanked by single strand RNA (Figure 6-8).

TGF- $\beta 1$ is a protein that controls proliferation, apoptosis, and other functions in most cells. The TGF- $\beta$ family is considered as a negative cell proliferation controller and positive apoptosis inducer. TGF- $\beta 1$ has been shown recently to increase the proliferation of variety of cells, for instance, airway smooth muscle cells, ${ }^{273}$ colon carcinoma cells, ${ }^{274,275}$ and wounded epidermal cells. ${ }^{276}$ TGF- $\beta 1$ is the most potent stimulus to HSCs in hepatic fibrosis. In quiescent HSCs, TGF- $\beta 1$ inhibits the proliferation. However, once the quiescent HSCs are activated by the positive feedback activation mechanism, TGF- $\beta 1$ loses its control to inhibit the proliferation of activated HSCs. ${ }^{277}$ Although the mechanisms of TGF- $\beta 1$ to induce ECM accumulation have not been fully understood, TGF- $\beta 1$ has been suggested to activate MAPK/ERK pathway, ${ }^{278}$ which is related closely to HSC proliferation. Although SMAD pathway has been reported to get activated after TGF- $\beta 1$ application, it was also revealed that SMAD2 pathway was not initiated by TGF- $\beta 1 .{ }^{275}$ Our results suggest that TGF- $\beta 1$ gene silencing inhibited the proliferation of HSC-T6 cells by MAPK/ERK pathway (Figure 6-3B). The role of TGF- $\beta 1$ on apoptosis was also changed after HSC activation. Saile et al. reported that DNA-NF- $\kappa B$ binding ability was inhibited by TGF- $\beta 1$ in activated HSCs ${ }^{279}$ which meant TGF- $\beta 1$ had antiapoptotic effect on fibrotic HSCs. Thus, the inhibition of TGF- $\beta 1$ allowed activated HSCs to undergo apoptosis partial (Figure 6-4).

In conclusion, effective HSC-specific TGF- $\beta 1$ gene silencing is possible using GFAP promoter which is a pol II promoter to modulate miR30-shRNA expression in the 


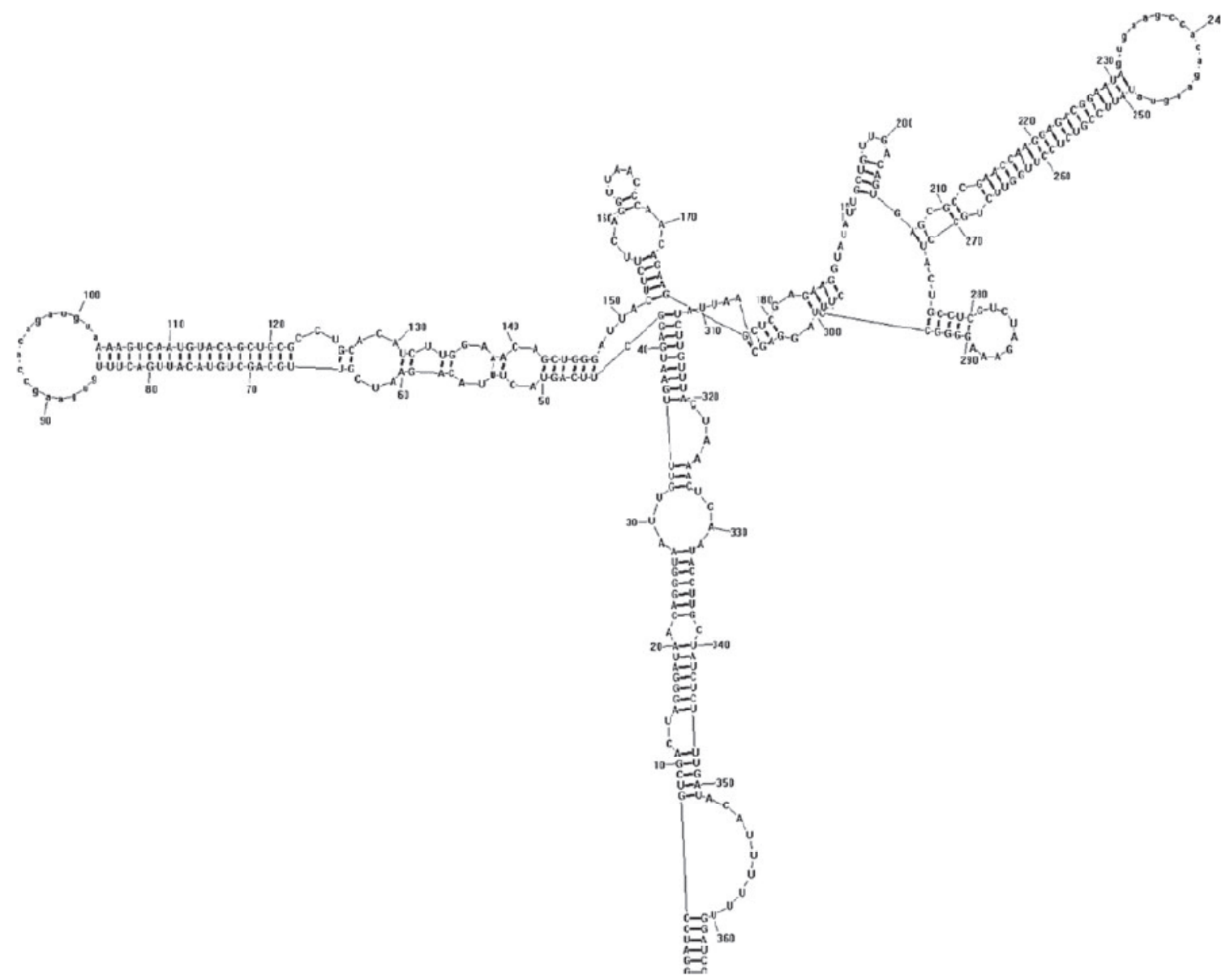

Figure 6-8 The prediction of the secondary structure of the pri-miRNA cluster mimic transcribed from pGFA-mi-shRNA-769+1033 plasmid. The arrows points to the single strands flanking the extended stem-loops of pri-miRNA cluster mimics. The ovals indicate the shRNA inserts. 
pri-miRNA mimic plasmids. The pGFA-mi-shRNA 769+1033 showed higher efficiency in reducing HSC TGF- $\beta 1$ mRNA level. 


\section{CHAPTER 7. SYSTEMIC ADMINISTRATION OF MICELLAR FORMULATED PTP, A NOVEL LPA ANTAGONIST, TO TREAT HEPATIC FIBROSIS}

\subsection{Introduction}

Hepatic fibrosis is excessive accumulation of ECM from an uncontrolled healing process leading to various liver injuries, such as viral infections, autoimmune reactions, etc. After hepatic damage, the connective tissue replaces injured hepatocytes, which prevents the regeneration of the liver. ${ }^{239}$ Fibrosis is also closely related the morbidity and mortality, including in cancer metastasis and chronic graft rejection. Damaged hepatocytes produce pro-inflammatory cytokines, such as TGF- $\beta 1$, and recruit inflammatory cells. Then, more pro-fibrotic mediators are secreted and results in the activation of $\alpha$-SMA expressing myofibroblasts. The major inducer of myofibroblast activation is TGF- $\beta 1$, which can be enhanced by IGF-1, bFGF, LPA etc.

Liver fibrosis has become a major problem on public health cost. However, efficient treatments that directly target fibrosis are lacking. So far, only pirfenidone has been evaluated in a Phase III trial. ${ }^{280}$ Treatment with this molecule decreases ECM accumulation in fibrotic liver. ${ }^{281}$ Nevertheless, pirfenidone also showed gastrointestinal adverse responses in a significant number of patients.

LPA is a growth factor-like mediator acting on G-protein-coupled receptors $(\mathrm{GPCR})^{282}$ presenting in various physiological fluids. LPA has been reported to involve in many diseases, such as cardiac ischemia, atheriosclerosis, obesity and hepatic fibrosis. The composition of LPA is long chain fatty acids, saturated (C18:0, C16:0) or unsaturated (C18:1, C20:4), linked to the glycerol backbone by acyl- or alkyl-group. At least six LPA receptors have been found. ${ }^{283}$ LPA1R, LPA2R and LPA3R are members of the endothelial differentiation gene family while LPA4R, LPA5R and LPA6R belong to the purinergic GPCR family. ${ }^{284}$ LPA1R is the most widely distributed subtypes throughout the organism. It is shown that LPA can activate hepatic myofibroblasts ${ }^{45}$ and increase the proliferation and survival of stellate cells. ${ }^{46,47}$ The correlations between plasma LPA concentration and the histological stages of liver fibrosis markers have been established in patients with chronic hepatitis $\mathrm{C}^{48,49}$

It is demonstrated that LPA1R antagonists to reduce the extent of fibrosis in kidney and lung. This result also provides a potential pharmacogical approach to treat liver fibrosis. Despite a growing number of LPAR antagonists have shown pharmacological ability, few of them have been validated for treatments in animals. It becomes very urgent that to develop more efficient anti-fibrotic drugs that may have important preclinical effects to imply the potent clinical applications in the management of liver fibrosis.

PTP, a novel synthesized LPA antagonist, showed significant inhibition effect on the proliferation of HSC-T6 cell line, which suggests the potent application on liver fibrosis therapeutics. However, the most difficult issue for PTP in vivo administration is the low water solubility. Surfactants, such as DMSO and Tween-80, have been commonly 
utilized to enhance the solubility for hydrophobic drugs. These surfactants also have their own problem, hepatic toxicity. To solve this problem, novel polymers synthesized by our group, poly(ethylene glycol)-b-poly(carbonate-co-lactide) copolymers, were used to make micellar formulation to enhance solubility.

In this chapter, PEG-PCcL micelles were applied to increase the aqueous solubility of PTP. After that, the anti-proliferation and anti-fibrosis abilities of this formulation were evaluated in vitro. Different doses of PTP loaded PEG-PCcL were intravenous administered to common bile duct ligated mice to check therapeutic effects on fibrosis in vivo.

\subsection{Material and Methods}

\subsubsection{Materials}

Goat anti-rat type I collagen and $\beta$-actin primary antibodies were purchased from Santa Cruz Biotech, Inc. (Santa Cruz, CA). Serum alanine transaminase (ALT) and aspartate transaminase (AST) kits were purchased from ID Labs, Inc (London, Canada). Citric acid and sodium citrate were procured from Curtin Matheson Scientific, Inc (Houston, TX). Sodium hydroxide was purchased from Fisher Scientific (Fair Lawn, NJ). Isoflurane was purchased from Baxter Pharmaceutical Products, Inc. (Deerfield, IL). All solvents and chemicals used in this study were used as available without further purification.

\subsubsection{In Vitro Application of PTP}

Cells were cultured in a 24-well plate to $60 \%$ confluence and treated with PTP dissolved in DMSO in various doses for $24 \mathrm{~h}$.

\subsubsection{MTT Assay}

HSC-T6 cells were seeded in 48 -well plate and reached $60 \%$ confluence before treated. After 24 hours treatment, the old medium was removed and $200 \mu \mathrm{L}$ medium with $0.5 \mathrm{mg} / \mathrm{mL}$ MTT was added. The cells were incubated for $1 \mathrm{~h}$ at $37^{\circ} \mathrm{C}$. After carefully removing the media again, $400 \mu \mathrm{L}$ of DMSO was added into each well to dissolve the formazan crystals. The absorbance was measured in a microplate reader at a wavelength of $560 \mathrm{~nm}$. Cell viability was expressed as the percentage of control group. 


\subsubsection{Determination of HSC-T6 Proliferation}

HSC-T6 cells were seeded in 6-well plate and reached 60\% confluence before treated. After 24 hours treatment and 1 hour before collection, BRDU was given to the cells. The proliferation of PTP treated HSC-T6 was determined by Cell Proliferation Assay Kit according to the manufacturer's protocol.

\subsubsection{Measurement of Caspase Activity}

Caspase-Glo 3/7 (Promega, Madison, WI) assay kit was used to determine the effect of PTP micelles on caspase-3/7 activity. Briefly, cell was seeded in 48-well plate and reached $60 \%$ confluence before treated. At $24 \mathrm{~h}$ post-treatment, supernant medium was moved and $100 \mu \mathrm{L}$ Caspase-Glo reagent was added to each well and incubated at room temperature for $1 \mathrm{~h}$. The contents were transferred into culture tubes, and luminescence was determined using a luminometer (Berthold, Germany).

\subsubsection{Preparation of Micelles}

PTP loaded micells were prepared by a film dispersion methods. ${ }^{285} 3 \mathrm{mg}$ PTP and 20 $\mathrm{mg}$ PEG-PCcL copolymer were dissolved in chloroform and a thin film was formed after removing organic solvent under reduced pressure. The resulting film then was hydrated and sonicated in $1 \mathrm{~mL}$ water. After that, the result formulation was centrifuged to remove un-encapsulated PTP and used at the same day.

\subsubsection{Determination of Drug Loading Efficiency}

To determine the PTP loading efficiency, un-encapsulated PTP during micelle preparation was dried and re-dissolved in dichloromethane. The drug concentration was measured by UV spectrometer and calculated according to the standard curve in various concentration of PTP in dichloromethane. The loading efficiency and density were calculated as previous publication. ${ }^{50}$

Drug loading efficiency=(Drug fed-Drug un-encapsulated $) /$ Drug fed

Drug loading density $=($ Drug fed-Drug un-encapsulated)/Polymer fed

\subsubsection{Particle Size Measurement}

The particle size distribution of micelles was determined by dynamic light scattering with Malvern Nano ZS. The intensity of scattered light was detected at $90^{\circ}$. 


\subsubsection{Animal Model and In Vivo Administration}

Male BALB/c mice weighing 20-25 g were purchased from Harlan Co. (San Diego, $\mathrm{CA}$ ) and were housed individually under the conditions as per the NIH (http://grants1.nih.gov/grants/olaw/references/phspol.html) and Institutional Animal Care and Use Committee (IACUC) using the approved protocols. All animals were housed in microisolator cages in virus-free facilities and fed laboratory chow and water ad labium.

Common bile duct ligation (CBDL) or sham operation was performed as described in our recent publication. ${ }^{222}$ In the first day of CBDL, animals were divided into 4 groups: sham (midline abdominal incision and closure), CBDL, CBDL+low dose PTP loaded micelles $(1 \mathrm{mg} / \mathrm{kg})$ and CBDL + high dose PTP loaded micelles $(10 \mathrm{mg} / \mathrm{kg})$. Micelle solution was given via the tail vein injection from the first day of CBDL three times a week for two consecutive weeks. At the end of experiment, blood was collected when animals were sacritificed under isoflurane anesthesia by puncture of the right heart vehicle and exsanguinations. Liver tissues were collected, washed, blotted dry and frozen.

\subsubsection{Standard Serum Parameters}

Traditional serum biomarkers for liver fibrosis, aspartate aminotransferase (AST) and alanine aminotransferase (ALT), were determined. Serum ALT and AST concentrations were measured using IDToxTM Alanine Transaminase color endpoint assay kit and IDToxTM Aspartate Transaminase Enzyme Assay Kit (ID LabsTM Inc, London, ON, Canada) according to the manufacturer's instructions and absorbance was measured using a spectrophotometer.

\subsubsection{Real Time PCR}

Total RNA was extracted from 20 mg liver tissue by RNAeasy Mini Kit and the RNA concentration was measured by UV spectrophotometry using Nanodrop spectronanometer (Thermo Scientific, Pittsburgh, PA). Then, $385 \mathrm{ng}$ of the total RNA per sample was reverse transcribed into cDNA using MultiScribe Reverse Transcriptase Reagent and random hexamers. The obtained cDNA was amplified by real time PCR using SYBR Green-1 dye universal master mix on ABI Prism 7700 Sequence Detection System (Applied Biosystems, Inc., Foster City, CA). The sequences of primers for detecting TGF- $\beta 1, \alpha$-SMA and $18 \mathrm{~S}$ as an internal control are the same as our previous report. ${ }^{22}$

\subsubsection{Hydroxyproline Assay}

$30 \mathrm{mg}$ liver tissues were hydrolyzd with $6 \mathrm{~N} \mathrm{HCl}$ and heating in an oven at $110^{\circ} \mathrm{C}$ for $18 \mathrm{~h}$. Then, citrate buffer and Chloramine-T reagent were added and allowed to react for 20 min at room temperature. Fresh prepared Ehrlich's reagent was then added to each 
sample and react for $15 \mathrm{~min}$ at a warm water bath $\left(60.8^{\circ} \mathrm{C}\right)$. Following cooling to room temperature, absorbance was read at $550 \mathrm{~nm}$ and the results were expressed as micrograms of hydroxyproline per grams of liver.

\subsubsection{Histological Staining}

Formalin-fixed liver specimens from mice in various groups were dehydrated in $70 \%$ alcohol, incubated in xylene, and embedded in paraffin. Five-micron-thick tissue sections were cut and stained with Masson's Trichrome staining for collagen.

\subsection{Results}

\subsubsection{Effects of PTP on HSC-T6 Cell Lines Proliferation and Apoptosis}

BRDU 1 hour pulse showed PTP inhibit HSC-T6 proliferation (Figure 7-1A). The green fluorescence indicated the cells which incorporated BRDU to the genome DNA during replication. MTT assay showed the same trend as BRDU assay (Figure 7-1B). However, PTP treated HSC-T6 cells did not show any inhibition on caspase activity (Figure 7-1C), which related to apoptosis. It suggests the decrease on cell viability only came from the down-regulation on proliferation.

\subsubsection{In Vitro Characterization of PTP in PEG-PCcL Micells}

PEG-PCcL ( $\mathrm{L}: \mathrm{C}=70: 30)$ micelles showed the least particle size in both no-PTP and PTP loaded situation, while PEG-PCcL ( $\mathrm{L}: \mathrm{C}=50: 50)$ and PEG-PCcL (L:C=30:70) micelles showed the slightly bigger in size (Table 7-1). After loading PTP, PEG-PCcL $(\mathrm{L}: \mathrm{C}=50: 50)$ micelles showed smaller size, but PEG-PCcL $(\mathrm{L}: \mathrm{C}=70: 30)$ micelles gave a much bigger size compare to their empty capsule. All of these micelles were stable for at least 5 days without significant change in particle size.

Under the same fed condition, PEG-PCcL $(\mathrm{L}: \mathrm{C}=50: 50)$ micelles showed the highest loading efficiency, while PEG-PCcL ( $\mathrm{L}: \mathrm{C}=30: 70)$ micelles showed the lowest (Table 7-2). The loading efficiency in PEG-PCcL $(\mathrm{L}: \mathrm{C}=50: 50)$ micelles can meet the requirement for in vivo administration.

\subsubsection{Blood Chemistry}

The level of traditional liver disease markers, ALT and AST, were determined (Figure 7-2). Administration of PTP loaded micelles in a dose of $1 \mathrm{mg} / \mathrm{kg}$ did not show any relief on both ALT and AST level. The dose of $10 \mathrm{mg} / \mathrm{kg}$ resulted in decreased levels of ALT and AST, which indicated the therapeutics on hepatic fibrosis. 
A
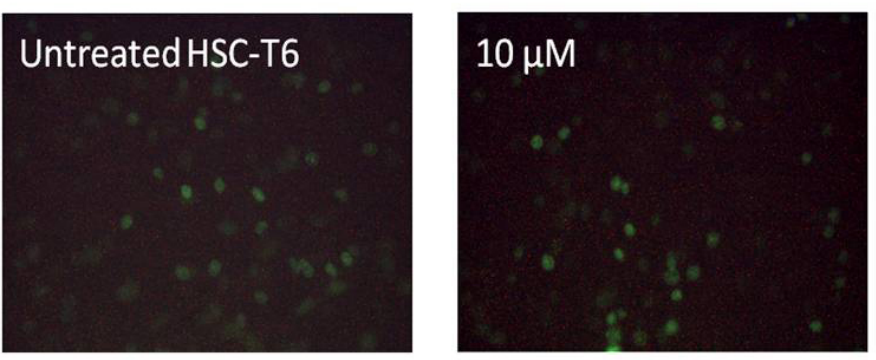

\section{$100 \mu \mathrm{M}$}

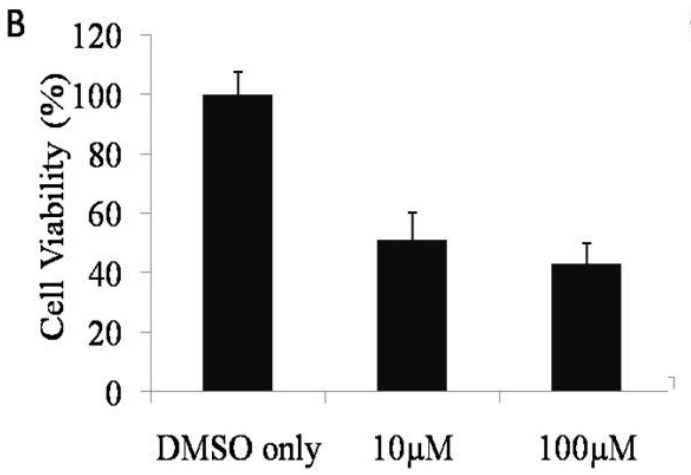

C

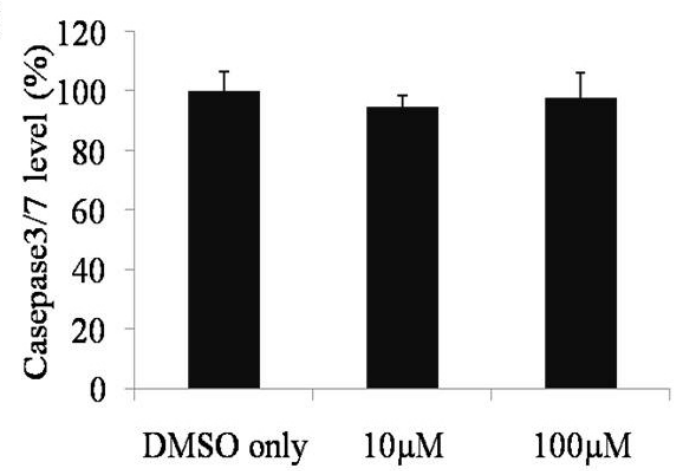

Figure 7-1 Effects of PTP on HSC-T6 proliferation and apoptosis. A) BRDU 1 hour pulse, green fluorescence indicated the cells which incorporated BRDU to the genome DNA during replication, B) MTT assay, demonstrating cell viability, C) caspase-3/7 activity assay. 
Table 7-1 Particle size measurement of empty and PTP loaded PEG-PCcL micelles.

\begin{tabular}{ccccccc}
\hline Polymer & $\begin{array}{c}\text { Z-Ave } \\
(\mathrm{nm})\end{array}$ & PDI & $\begin{array}{c}\text { PK1 mean } \\
(\mathrm{nm})\end{array}$ & $\begin{array}{c}\text { PK2 mean } \\
(\mathrm{nm})\end{array}$ & $\begin{array}{c}\text { PK1 } \\
(\%)\end{array}$ & $\begin{array}{c}\text { PK1 } \\
(\%)\end{array}$ \\
\hline PEG-PCcL (L:C=70:30) & 102 & 0.257 & 159 & 42.4 & 78.4 & 21.6 \\
PEG-PCcL (L:C=70:30) LPA-ANT & 106 & 0.256 & 132 & 39.5 & 85.2 & 92.8 \\
PEG-PCcL (L:C=50:50) & 127 & 0.156 & 155 & 48.3 & 84.3 & 7.2 \\
PEG-PCcL (L:C=50:50) LPA-ANT & 122 & 0.168 & 158 & 54.4 & 83.6 & 15.7 \\
PEG-PCcL (L:C=30:70) & 124 & 0.210 & 172 & 42.0 & & 92.8 \\
PEG-PCcL (L:C=30:70) LPA-ANT & 141 & 0.211 & 169 & & & 7.2 \\
\hline
\end{tabular}


Table 7-2 Micelle loading efficiency and loading density.

\begin{tabular}{cccc}
\hline Polymer & Drug concentration $(\mathrm{mg} / \mathrm{mL})$ & Loading efficiency $(\%)$ & Loading density $(\%)$ \\
\hline PEG-PCcL (L:C=70:30) & 0.99 & 33 & 5.0 \\
PEG-PCcL (L:C=50:50) & 1.70 & 57 & 8.5 \\
PEG-PCcL (L:C=30:70) & 0.48 & 17 & 2.5 \\
\hline
\end{tabular}


A

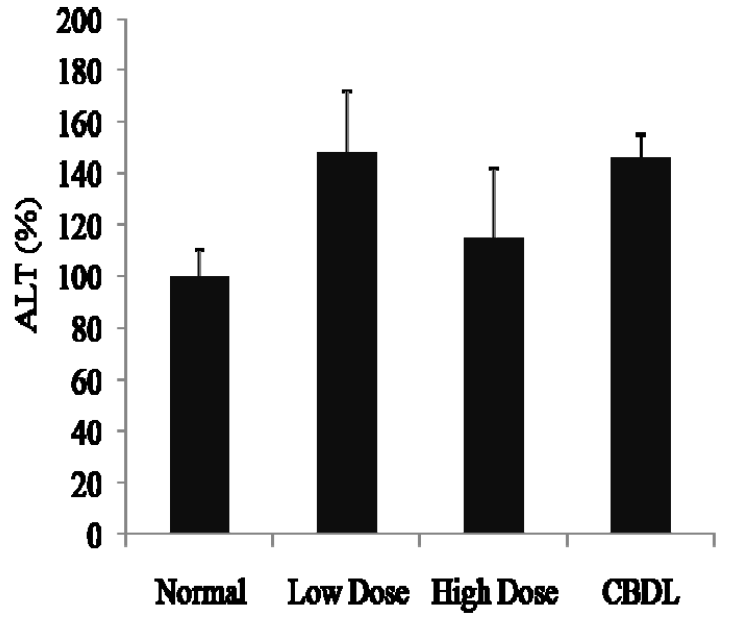

B

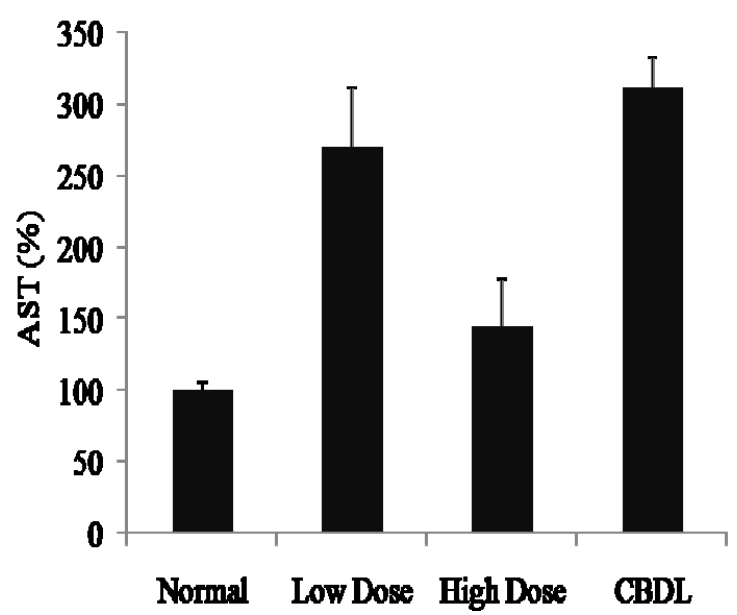

Figure 7-2 The level of traditional liver disease markers, ALT and AST, were determined. A) ALT level, B) AST level. 


\subsubsection{High Dose PTP Micelles Inhibits Both Profibrogens and Myofibroblast Activation}

TGF- $\beta 1$ is a potent fibrogenic cytokine believed to regulate fibrosis. Both low dose and high dose treated group showed significant decrease on hepatic TGF- $\beta 1$ gene expression (Figure 7-3A).

The progression of liver fibrosis enlarge the pool of the cells, which can express $\alpha$-smooth muscle actin ( $\alpha$-SMA) ${ }^{222}$ Therefore, we determined $\alpha$-SMA concentration in mRNA level before and after treatment. Only high dose treatment significantly decreased $\alpha$-SMA mRNA synthesis in CBDL mice (Figure 7-3B). Low dose group did not show significant inhibition on $\alpha$-SMA mRNA production.

\subsubsection{Collagen Levels}

To confirm therapeutic effects of PTP on inhibition of collagen production, Masson's trichrome staining was applied to mouse liver tissues. In sham operated mice, collagen expression was very low (Figure 7-4A). In rats administrated with PTP on a dose of $10 \mathrm{mg} / \mathrm{kg}$, there was a significant decrease in collagen deposition compared to CBDL mice.

The level of type I collagen gene expression, the major component in ECM, was also determined by Western blot analysis (Figure 7-4B). Only high dose treated group showed inhibition on type I collagen expression, with relatively higher level of collagen in low dose group.

To quantify the total collagen level, the liver tissue was measured by hydroxyproline assay (Figure 7-4C). There was significant increase in hydroxyproline content in the liver after CBDL, suggesting the liver fibrosis model was successfully established. Hydroxyproline content of the livers showed significant decrease in high dose treated group compared to CBDL group.

\subsection{Discussion}

In various organs in animals and humans, fibrosis is associated with increased LPA production or of some LPA receptor, mainly LPA1R, expression. ${ }^{286}$ Treatment of animal models with specific antagonists clearly indicates the contribution of LPA to the development of kidney, lung, vascular and dermal fibrosis. The involvement of LPA antagonist in liver fibrosis treatment is also strongly suspected but still unproven.

In our experiment, it was clearly shown that the LPA antagonist, PTP, inhibited the proliferation of hepatic stellate cells in vitro. The drug works in concentration of both 10 $\mu \mathrm{M}$ and $100 \mu \mathrm{M}$. There was no significant difference between $10 \mu \mathrm{M}$ and $100 \mu \mathrm{M}$ treated groups. MTT assay showed the same trend after measuring the cell viability. However, 

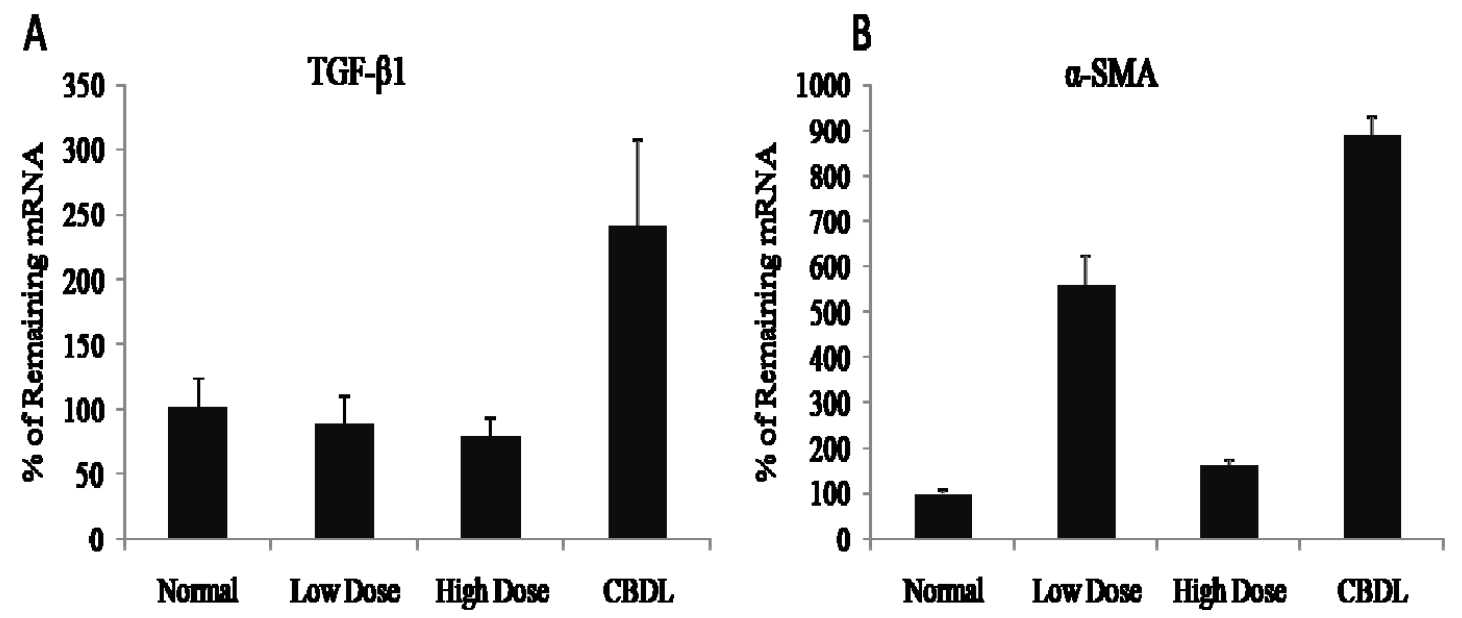

Figure 7-3 mRNA expression level of fibrotic related factors. A) mRNA level of TGF- $\beta 1$, B) mRNA level of $\alpha$-SMA. 


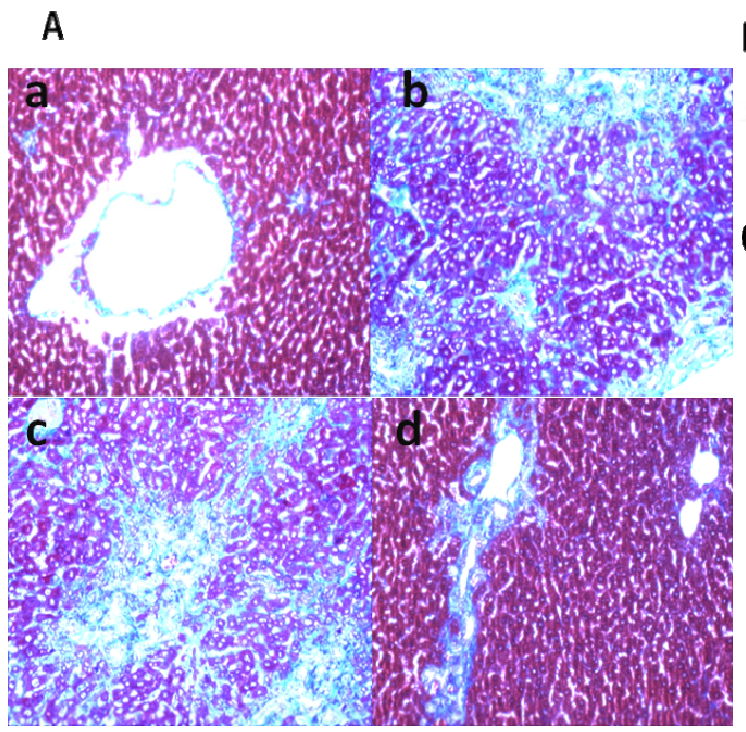

\section{B}

PC1 NC1 HD1 LD1 PC2 NC2 HD2 LD2 HD3

c

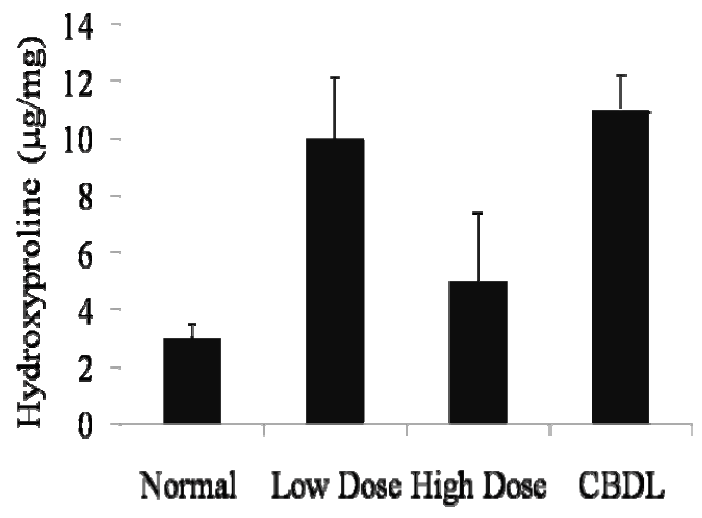

Figure 7-4 Measurement of type I collagen and total collagen. A) Mason's Trichrome staining for total collagen, a) non-fibrotic mice, b) CBDL mice, c) low dose group, d) high dose group. B) Western blot for type I collagen, upper is type I collagen, lower is $\beta$ actin, $\mathrm{PC}$ means $\mathrm{CBDL}$ mice, $\mathrm{NC}$ means normal mice, LD means low dose group and HD means high dose group. C) Hydroxyproline level of total collagen. 
the caspase-3/7 activity did not show any inhibition or activation from these two treated groups. These results may suggest PTP only affect the proliferation process in liver fibrosis. Inhibition on proliferation in HSC-T6 cell line gave us the hint to slow down the HSCs activation by this strategy. Inhibition of proliferation may have the similar results as activation of apoptosis in vitro. However, it is totally different in vivo. Induction of apoptosis in vivo may create disaster during treating procedure, while control on proliferation has fewer problems. ${ }^{287}$

Many potent drugs have the same problem, low aqueous solubility, to prevent their practical application in vivo. Polymeric micelles are capable of preventing drug degradation and have the potential of targeted delivery after modification to avoid adverse effects. ${ }^{288}$ In this study, PEG-PCcL copolymers were utilized to make micelle delivery system to enhance the solubility of PTP. The maximum solubility by formulated with $20 \mathrm{mg}$ PEG-PCcL ( $\mathrm{L}: \mathrm{C}=50: 50$ ) reached to $1.7 \mathrm{mg} / \mathrm{mL}$. Polymeric micelles enhance solubilization of hydrophobic compounds by accommodating them in their hydrophobic core of polymer. When the drugs are loaded, the size of micelles may change compared to empty ones. PTP loaded PEG-PCcL ( $\mathrm{L}: \mathrm{C}=30: 70)$ micelles increase particle size to almost $115 \%$ and PTP loaded PEG-PCcL ( $\mathrm{L}: \mathrm{C}=70: 30)$ micelles also increase particle size slightly. Whereas, PTP loaded PEG-PCcL ( $\mathrm{L}: \mathrm{C}=50: 50)$ micelles decrease the particle after formulation, which may suggest the higher compatibility between PEG-PCcL $(\mathrm{L}: \mathrm{C}=50: 50)$ and PTP. This higher compatibility can enhance the drug loading efficiency and make the whole delivery system more shrinkable.

LPA antagonist used as an agent to treat liver fibrosis is applicable if reasonable aqueous solubility can be reached. The control on proliferation is much easier to handle to than the control on apoptosis in treating fibrotic disease. PTP showed good therapeutic effects both in vitro and in vivo, which may act as an excellent drug to treat hepatic fibrosis in the future. 


\section{LIST OF REFERENCES}

1. McCaughan GW, Gorrell MD, Bishop GA, Abbott CA, Shackel NA, McGuinness PH, Levy MT, Sharland AF, Bowen DG, Yu D: Molecular pathogenesis of liver disease: An approach to hepatic inflammation, cirrhosis and liver transplant tolerance, Immunological Reviews 2000, 174:172-191

2. Malhi H, Gores GJ, Lemasters JJ: Apoptosis and necrosis in the liver: A tale of two deaths, Hepatology 2006, 43:S31-S44

3. Lemasters JJ, Ji S, Thurman RG: Centrilobular injury following hypoxia in isolated, perfused rat liver, Science 1981, 213:661-663

4. Faubion WA, Guicciardi ME, Miyoshi H, Bronk SF, Roberts PJ, Svingen PA, Kaufmann SH, Gores GJ: Toxic bile salts induce rodent hepatocyte apoptosis via direct activation of Fas, Journal of Clinical Investigation 1999, 103:147-154

5. Wu CH, Walton $\mathrm{CM}, \mathrm{Wu} \mathrm{GY}$ : Targeted inhibition of type I procollagen synthesis by antisense DNA oligonucleotides, Gene Therapy and Regulation 2000, 1:193205

6. Casini A, Ceni E, Salzano R, Biondi P, Parola M, Galli A, Foschi M, Caligiuri A, Pinzani M, Surrenti C: Neutrophil derived superoxide anion induces lipid peroxidation and stimulates collagen synthesis in human hepatic stellate cells: Role of nitric oxide, Hepatology 1997, 25:361-367

7. Patel T, Roberts LR, Jones BA, Gores GJ: Dysregulation of apoptosis as a mechanism of liver disease: An overview, Seminars in Liver Disease 1998, 18:105-114

8. Canbay A, Higuchi H, Bronk SF, Taniai M, Sebo TJ, Gores GJ: Fas enhances fibrogenesis in the bile duct ligated mouse: A link between apoptosis and fibrosis, Gastroenterology 2002, 123:1323-1330

9. Pietrangelo A, Gualdi R, Casalgrandi G, Montosi G, Ventura E: Molecular and cellular aspects of iron-induced hepatic cirrhosis in rodents, Journal of Clinical Investigation 1995, 95:1824-1831

10. Brady LM, Beno D, Davis BH: Bile acid stimulation of early growth response gene and mitogen-activated protein kinase is protein kinase $\mathrm{C}$-dependent, Biochemical Journal 1996, 316:765-769 
11. Nieto N, Greenwel P, Friedman SL, Zhang F, Dannenberg AJ, Cederbaum AI: Ethanol and arachidonic acid increase 2 (I) Collagen expression in rat hepatic stellate cells overexpressing cytochrome P450 2E1, Journal of Biological Chemistry 2000, 275:20136-20145

12. Fadok VA, Bratton DL, Konowal A, Freed PW, Westcott JY, Henson PM: Macrophages that have ingested apoptotic cells in vitro inhibit proinflammatory cytokine production through autocrine/paracrine mechanisms involving TGF-beta, PGE2, and PAF, Journal of Clinical Investigation 1998, 101:890-898

13. Kurosaka K, Watanabe N, Kobayashi Y: Production of proinflammatory cytokines by resident tissue macrophages after phagocytosis of apoptotic cells, Cellular Immunology 2001, 211:1-7

14. Brenzel A, Gressner AM: Characterization of insulin-like growth factor (IGF)-I receptor binding sites during in vitro transformation of rat hepatic stellate cells to myofibroblasts, Clinical Chemistry and Laboratory Medicine 1996, 34:401-410

15. Pinzani M, Abboud HE, Aron DC: Secretion of insulin-like growth factor-I and binding proteins by rat liver fat-storing cells: Regulatory role of platelet-derived growth factor, Endocrinology 1990, 127:2343-2349

16. Issa R, Williams E, Trim N, Kendall T, Arthur M, Reichen J, Benyon R, Iredale J: Apoptosis of hepatic stellate cells: Involvement in resolution of biliary fibrosis and regulation by soluble growth factors, Gut 2001, 48:548-557

17. Saile B, Matthens N, Knittel T, Ramadori G: Transforming growth factor and tumor necrosis factor inhibit both apoptosis and proliferation of activated rat hepatic stellate cells, Hepatology 1999, 30:196-202

18. Issa R, Zhou X, Trim N, Millward-Sadler H, Krane S, Benyon C, Iredale J: Mutation in collagen-1 that confers resistance to the action of collagenase results in failure of recovery from CCl4-induced liver fibrosis, persistence of activated hepatic stellate cells, and diminished hepatocyte regeneration, The FASEB Journal 2003, 17:47-49

19. Guedez L, Stetler-Stevenson WG, Wolff L, Wang J, Fukushima P, Mansoor A, Stetler-Stevenson M: In vitro suppression of programmed cell death of B cells by tissue inhibitor of metalloproteinases-1, Journal of Clinical Investigation 1998, 102:2002-2010

20. Benyon RC, Hovell CJ, Da Gaca M, Jones EH, Iredale JP, Arthur MJP: Progelatinase A is produced and activated by rat hepatic stellate cells and promotes their proliferation, Hepatology 1999, 30:977-986 
21. Arthur MJP: Fibrogenesis II: Metalloproteinases and their inhibitors in liver fibrosis, American Journal of Physiology-Gastrointestinal and Liver Physiology 2000, 279:G245-G249

22. Friedman SL, Roll FJ, Boyles J, Bissell DM: Hepatic lipocytes: The principal collagen-producing cells of normal rat liver, Proceedings of the National Academy of Sciences of the United States of America 1985, 82:8681-8685

23. Friedman SL: The cellular basis of hepatic fibrosis: Mechanisms and treatment strategies, New England Journal of Medicine 1993, 328:1828-1835

24. Maher JJ, McGuire RF: Extracellular matrix gene expression increases preferentially in rat lipocytes and sinusoidal endothelial cells during hepatic fibrosis in vivo, Journal of Clinical Investigation 1990, 86:1641-1648

25. Pinzani M: Liver fibrosis, Springer Seminars in Immunopathology 1999, 21:475490

26. Friedman SL: Hepatic stellate cells: Protean, multifunctional, and enigmatic cells of the liver, Physiological Reviews 2008, 88:125-172

27. Yang N, Ye Z, Li F, Mahato RI: HPMA polymer-based site-specific delivery of oligonucleotides to hepatic stellate cells, Bioconjugate Chemistry 2009, 20:213221

28. Gressner AM, Weiskirchen R, Breitkopf K, Dooley S: Roles of TGF-beta in hepatic fibrosis, Frontiers in Bioscience 2002, 7:D793-D807

29. Cheng K, Yang N, Mahato RI: TGF-beta1 gene silencing for treating liver fibrosis, Molecular Pharmaceutics 2009, 6:772-779

30. Yang N, Mahato RI: GFAP promoter-driven RNA interference on TGF-betal to treat liver fibrosis, Pharmaceutical Research 2011, 28:752-761

31. Rogers FA, Lloyd JA, Glazer PM: Triplex-forming oligonucleotides as potential tools for modulation of gene expression, Current Medicinal Chemistry: Anti-Cancer Agents 2005, 5:319-326

32. Casey BP, Glazer PM: Gene targeting via triple-helix formation, Progress in Nucleic Acid Research and Molecular Biology 2001, 67:163-192

33. Letai AG, Palladino MA, Fromm E, Rizzo V, Fresco JR: Specificity in formation of triple-stranded nucleic acid helical complexes: Studies with agarose-linked polyribonucleotide affinity columns, Biochemistry 1988, 27:9108-9112 
34. Ye Z, Guntaka RV, Mahato RI: Sequence-specific triple helix formation with genomic DNA, Biochemistry 2007, 46:11240-11252

35. Joseph J, Kandala JC, Veerapanane D, Weber KT, Guntaka RV: Antiparallel polypurine phosphorothioate oligonucleotides form stable triplexes with the rat type 1 (I) collagen gene promoter and inhibit transcription in cultured rat fibroblasts, Nucleic Acids Research 1997, 25:2182-2188

36. Nakanishi M, Guntaka RV, Weber KT: Triple helix formation with the promoter of human 1 (I) procollagen gene by an antiparallel triplex-forming oligodeoxyribonucleotide, Nucleic Acids Research 1998, 26:5218-5222

37. Wooldridge JE, Ballas Z, Krieg AM, Weiner GJ: Immunostimulatory oligodeoxynucleotides containing $\mathrm{CpG}$ motifs enhance the efficacy of monoclonal antibody therapy of lymphoma, Blood 1997, 89:2994-2998

38. Cheng K, Ye Z, Guntaka RV, Mahato RI: Biodistribution and hepatic uptake of triplex-forming oligonucleotides against type 1 (I) collagen gene promoter in normal and fibrotic rats, Molecular Pharmaceutics 2005, 2:206-217

39. Yu RZ, Geary RS, Monteith DK, Matson J, Truong L, Fitchett J, Levin AA: Tissue disposition of 2-O-(2-methoxy)-ethyl modified antisense oligonucleotides in monkeys, Journal of Pharmaceutical Sciences 2004, 93:48-59

40. Agrawals S, Temsamani J, Galbraith W, Tang J: Pharmacokinetics of antisense oligonucleotides, Clinical Pharmacokinetics 1995, 28:7-16

41. Ye Z, Cheng K, Guntaka RV, Mahato RI: Targeted delivery of a triplex-forming oligonucleotide to hepatic stellate cells, Biochemistry 2005, 44:4466-4476

42. Bissell D, Wang SS, Jarnagin W, Roll FJ: Cell-specific expression of transforming growth factor-beta in rat liver: Evidence for autocrine regulation of hepatocyte proliferation, Journal of Clinical Investigation 1995, 96:447-455

43. Gressner A: Cytokines and cellular crosstalk involved in the activation of fat-storing cells, Journal of Hepatology 1995, 22:28-36

44. Kim KH, Kim HC, Hwang MY, Oh HK, Lee TS, Chang YC, Song HJ, Won NH, Park KK: The antifibrotic effect of TGF-betal siRNAs in murine model of liver cirrhosis, Biochemical and Biophysical Research Communications 2006, 343:1072-1078

45. Tangkijvanich P, Melton AC, Chitapanarux T, Han J, Yee HF: Platelet-derived growth factor-BB and lysophosphatidic acid distinctly regulate hepatic myofibroblast migration through focal adhesion kinase, Experimental Cell Research 2002, 281:140-147 
46. Ikeda H, Yatomi Y, Yanase M, Satoh H, Nishihara A, Kawabata M, Fujiwara K: Effects of lysophosphatidic acid on proliferation of stellate cells and hepatocytes in culture, Biochemical and Biophysical Research Communications 1998, $248: 436-440$

47. Ikeda H, Nagashima K, Yanase M, Tomiya T, Arai M, Inoue Y, Tejima K, Nishikawa T, Omata M, Kimura S: Involvement of Rho/Rho kinase pathway in regulation of apoptosis in rat hepatic stellate cells, American Journal of Physiology-Gastrointestinal and Liver Physiology 2003, 285:G880-G886

48. Watanabe N, Ikeda H, Nakamura K, Ohkawa R, Kume Y, Aoki J, Hama K, Okudaira S, Tanaka M, Tomiya T: Both plasma lysophosphatidic acid and serum autotaxin levels are increased in chronic hepatitis C, Journal of Clinical Gastroenterology 2007, 41:616-623

49. Watanabe N, Ikeda H, Nakamura K, Ohkawa R, Kume Y, Tomiya T, Tejima K, Nishikawa T, Arai M, Yanase M: Plasma lysophosphatidic acid level and serum autotaxin activity are increased in liver injury in rats in relation to its severity, Life Sciences 2007, 81:1009-1015

50. Danquah M, Fujiwara T, Mahato RI: Self-assembling methoxypoly (ethylene glycol)-b-poly (carbonate-co-L-lactide) block copolymers for drug delivery, Biomaterials 2010, 31:2358-2370

51. Kim SH, Jeong JH, Lee SH, Kim SW, Park TG: Local and systemic delivery of VEGF siRNA using polyelectrolyte complex micelles for effective treatment of cancer, Journal of Controlled Release 2008, 129:107-116

52. Ptasznik A, Nakata Y, Kalota A, Emerson SG, Gewirtz AM: Short interfering RNA (siRNA) targeting the Lyn kinase induces apoptosis in primary, and drug resistant, BCR-ABL1(+) leukemia cells, Nature Medicine 2004, 10:1187-1189

53. Xia CF, Zhang Y, Zhang Y, Boado RJ, Pardridge WM: Intravenous siRNA of brain cancer with receptor targeting and avidin-biotin technology, Pharmaceutical Research 2007, 24:2309-2316

54. Morrissey DV, Lockridge JA, Shaw L, Blanchard K, Jensen K, Breen W, Hartsough K, Machemer L, Radka S, Jadhav V, Vaish N, Zinnen S, Vargeese C, Bowman K, Shaffer CS, Jeffs LB, Judge A, MacLachlan I, Polisky B: Potent and persistent in vivo anti-HBV activity of chemically modified siRNAs, Nature Biotechnology 2005, 23:1002-1007

55. Okumura A, Pitha PM, Harty RN: ISG15 inhibits Ebola VP40 VLP budding in an L-domain-dependent manner by blocking Nedd4 ligase activity, Proceedings of the National Academy of Sciences of the United States of America 2008, 105:3974-3979 
56. Sharma HW, Narayanan R: The therapeutic potential of antisense oligonucleotides, Bioessays 1995, 17:1055-1063

57. Orr RM: Technology evaluation: Fomivirsen, Isis Pharmaceuticals Inc/CIBA vision, Current Opinion in Molecular Therapeutics 2001, 3:288-294

58. Marwick C: First "antisense" drug will treat CMV retinitis, JAMA: The Journal of the American Medical Association 1998, 280:871

59. Loke SL, Stein CA, Zhang XH, Mori K, Nakanishi M, Subasinghe C, Cohen JS, Neckers LM: Characterization of oligonucleotide transport into living cells, Proceedings of the National Academy of Sciences of the United States of America 1989, 86:3474-3478

60. Breslauer KJ, Frank R, Blocker H, Marky LA: Predicting DNA duplex stability from the base sequence, Proceedings of the National Academy of Sciences of the United States of America 1986, 83:3746-3750

61. Boiziau C, Kurfurst R, Cazenave C, Roig V, Thuong NT, Toulme JJ: Inhibition of translation initiation by antisense oligonucleotides via an RNase-H independent mechanism, Nucleic Acids Research 1991, 19:1113-1119

62. Walder RY, Walder JA: Role of RNase H in hybrid-arrested translation by antisense oligonucleotides, Proceedings of the National Academy of Sciences of the United States of America 1988, 85:5011-5015

63. Bonham MA, Brown S, Boyd AL, Brown PH, Bruckenstein DA, Hanvey JC, Thomson SA, Pipe A, Hassman F, Bisi JE, et al.: An assessment of the antisense properties of RNase H-competent and steric-blocking oligomers, Nucleic Acids Research 1995, 23:1197-1203

64. Rogers FA, Lloyd JA, Glazer PM: Triplex-forming oligonucleotides as potential tools for modulation of gene expression, Current Medicinal Chemistry Anticancer Agents 2005, 5:319-326

65. Casey BP, Glazer PM: Gene targeting via triple-helix formation, Progress in Nucleic Acid Research and Molecular Biology 2001, 67:163-192

66. Letai AG, Palladino MA, Fromm E, Rizzo V, Fresco JR: Specificity in formation of triple-stranded nucleic acid helical complexes: Studies with agarose-linked polyribonucleotide affinity columns, Biochemistry 1988, 27:9108-9112

67. Ye Z, Guntaka RV, Mahato RI: Sequence-specific triple helix formation with genomic DNA, Biochemistry 2007, 46:11240-11252 
68. Maher LJ, 3rd: Prospects for the therapeutic use of antigene oligonucleotides, Cancer Investigation 1996, 14:66-82

69. Praseuth D, Guieysse AL, Helene C: Triple helix formation and the antigene strategy for sequence-specific control of gene expression, Biochimica et Biophysica Acta 1999, 1489:181-206

70. Yamamoto S, Yamamoto T, Shimada S, Kuramoto E, Yano O, Kataoka T, Tokunaga T: DNA from bacteria, but not from vertebrates, induces interferons, activates natural killer cells and inhibits tumor growth, Microbiology and Immunology 1992, 36:983-997

71. Krieg AM: Lymphocyte activation by $\mathrm{CpG}$ dinucleotide motifs in prokaryotic DNA, Trends in Microbiology 1996, 4:73-76

72. Krieg AM: Immune effects and mechanisms of action of $\mathrm{CpG}$ motifs, Vaccine 2000, 19:618-622

73. Redford TW, Yi AK, Ward CT, Krieg AM: Cyclosporin A enhances IL-12 production by CpG motifs in bacterial DNA and synthetic oligodeoxynucleotides, The Journal of Immunology 1998, 161:3930-3935

74. Yi AK, Klinman DM, Martin TL, Matson S, Krieg AM: Rapid immune activation by $\mathrm{CpG}$ motifs in bacterial DNA. Systemic induction of IL-6 transcription through an antioxidant-sensitive pathway, The Journal of Immunology 1996, 157:5394-5402

75. Krieg AM: CpG motifs in bacterial DNA and their immune effects, Annual Review of Immunology 2002, 20:709-760

76. Hartmann G, Weiner G, Krieg A: CpG DNA: A potent signal for growth, activation, and maturation of human dendritic cells, Proceedings of the National Academy of Sciences of the United States of America 1999, 96:9305-9310

77. Barchet W, Wimmenauer V, Schlee M, Hartmann G: Accessing the therapeutic potential of immunostimulatory nucleic acids, Current Opinion in Immunology 2008, 20:389-395

78. Kanzler H, Barrat FJ, Hessel EM, Coffman RL: Therapeutic targeting of innate immunity with Toll-like receptor agonists and antagonists, Nature Medicine 2007, $13: 552-559$

79. Fonseca DE, Kline JN: Use of CpG oligonucleotides in treatment of asthma and allergic disease, Advanced Drug Delivery Reviews 2009, 61:256-262 
80. Doudna JA, Cech TR: The chemical repertoire of natural ribozymes, Nature 2002, 418:222-228

81. Doherty EA, Doudna JA: Ribozyme structures and mechanisms, Annual Review of Biophysics Biomolecular Structure 2001, 30:457-475

82. Puerta-Fernandez E, Romero-Lopez C, Barroso-delJesus A, Berzal-Herranz A: Ribozymes: Recent advances in the development of RNA tools, FEMS Microbiology Reviews 2003, 27:75-97

83. Li QX, Tan P, Ke N, Wong-Staal F: Ribozyme technology for cancer gene target identification and validation, Advances in Cancer Research 2006, 96:103-143

84. Akashi H, Matsumoto S, Taira K: Gene discovery by ribozyme and siRNA libraries, Nature Reviews Molecular Cell Biology 2005, 6:413-422

85. Khan AU: Ribozyme: A clinical tool, Clinica Chimica Acta 2006, 367:20-27

86. Mannironi C, Di Nardo A, Fruscoloni P, Tocchini-Valentini G: In vitro selection of dopamine RNA ligands, Biochemistry 1997, 36:9726-9734

87. Nieuwlandt D, Wecker M, Gold L: In vitro selection of RNA ligands to substance P, Biochemistry 1995, 34:5651-5659

88. Geiger A, Burgstaller P, von der Eltz H, Roeder A, Famulok M: RNA aptamers that bind L-arginine with sub-micromolar dissociation constants and high enantioselectivity, Nucleic Acids Research 1996, 24:1029-1036

89. Lupold SE, Hicke BJ, Lin Y, Coffey DS: Identification and characterization of nuclease-stabilized RNA molecules that bind human prostate cancer cells via the prostate-specific membrane antigen, Cancer Research 2002, 62:4029-4033

90. Nahvi A, Sudarsan N, Ebert MS, Zou X, Brown KL, Breaker RR: Genetic control by a metabolite binding mRNA, Chemistry and Biology 2002, 9:1043

91. Winkler W, Nahvi A, Breaker RR: Thiamine derivatives bind messenger RNAs directly to regulate bacterial gene expression, Nature 2002, 419:952-956

92. Winkler WC, Cohen-Chalamish S, Breaker RR: An mRNA structure that controls gene expression by binding FMN, Proceedings of the National Academy of Sciences of the United States of America 2002, 99:15908-15913

93. Tomita N, Kashihara N, Morishita R: Transcription factor decoy oligonucleotide based therapeutic strategy for renal disease, Clinical and Experimental Nephrology 2007, 11:7-17 
94. Morishita R, Gibbons GH, Horiuchi M, Ellison KE, Nakama M, Zhang L, Kaneda Y, Ogihara T, Dzau VJ: A gene therapy strategy using a transcription factor decoy of the E2F binding site inhibits smooth muscle proliferation in vivo, Proceedings of the National Academy of Sciences of the United States of America 1995, 92:5855-5859

95. Romanelli A, Pedone C, Saviano M, Bianchi N, Borgatti M, Mischiati C, Gambari R: Molecular interactions with nuclear factor kappaB (NF-kappaB) transcription factors of a PNA-DNA chimera mimicking NF-kappaB binding sites, European Journal of Biochemistry 2001, 268:6066-6075

96. Stojanovic T, Wagner AH, Wang S, Kiss E, Rockstroh N, Bedke J, Grone HJ, Hecker M: STAT-1 decoy oligodeoxynucleotide inhibition of acute rejection in mouse heart transplants, Basic Research in Cardiology 2009, 104:719-729

97. De Croos J, Pilliar R, Kandel R: AP-1 DNA binding activity regulates the cartilage tissue remodeling process following cyclic compression in vitro, Biorheology 2008, 45:459-469

98. Cho-Chung YS: CRE-enhancer DNA decoy: A tumor target-based genetic tool, Annals of the New York Academy of Sciences 2003, 1002:124-133

99. Pontes O, Li CF, Nunes PC, Haag J, Ream T, Vitins A, Jacobsen SE, Pikaard CS: The Arabidopsis chromatin-modifying nuclear siRNA pathway involves a nucleolar RNA processing center, Cell 2006, 126:79-92

100. Lee YS, Nakahara K, Pham JW, Kim K, He Z, Sontheimer EJ, Carthew RW: Distinct roles for Drosophila Dicer-1 and Dicer-2 in the siRNA/miRNA silencing pathways, Cell 2004, 117:69-81

101. Matranga C, Tomari Y, Shin C, Bartel DP, Zamore PD: Passenger-strand cleavage facilitates assembly of siRNA into Ago2-containing RNAi enzyme complexes, Cell 2005, 123:607-620

102. Ameres SL, Martinez J, Schroeder R: Molecular basis for target RNA recognition and cleavage by human RISC, Cell 2007, 130:101-112

103. Hutvagner G, Zamore PD: A microRNA in a multiple-turnover RNAi enzyme complex, Science 2002, 297:2056-2060

104. Overhoff M, Sczakiel G: Phosphorothioate-stimulated uptake of short interfering RNA by human cells, EMBO Reports 2005, 6:1176-1181

105. Stein C: Phosphorothioate antisense oligodeoxynucleotides: Questions of specificity, Trends in Biotechnology 1996, 14:147-149 
106. Amarzguioui M, Holen T, Babaie E, Prydz H: Tolerance for mutations and chemical modifications in a siRNA, Nucleic Acids Research 2003, 31:589-595

107. Braasch DA, Jensen S, Liu Y, Kaur K, Arar K, White MA, Corey DR: RNA interference in mammalian cells by chemically-modified RNA, Biochemistry 2003, 42:7967-7975

108. Chiu YL, Rana TM: siRNA function in RNAi: A chemical modification analysis, RNA 2003, 9:1034-1048

109. Connolly BA, Eckstein F, Grotjahn L: Direct mass spectroscopic method for determination of oxygen isotope position in adenosine 5'-O-(1-thiotriphosphate): Determination of the stereochemical course of the yeast phenylalanyl-tRNA synthetase reaction, Biochemistry 1984, 23:2026-2031

110. Cain K, Partis MD, Griffiths DE: Dibutylchloromethyltin chloride, a covalent inhibitor of the adenosine triphosphate synthase complex, The Biochemical Journal 1977, 166:593-602

111. Lato SM, Ozerova ND, He K, Sergueeva Z, Shaw BR, Burke DH: Boron containing aptamers to ATP, Nucleic Acids Research 2002, 30:1401-1407

112. Porter KW, Briley JD, Shaw BR: Direct PCR sequencing with boronated nucleotides, Nucleic Acids Research 1997, 25:1611-1617

113. Shaw BR, Dobrikov M, Wang X, Wan J, He K, Lin JL, Li P, Rait V, Sergueeva ZA, Sergueev D: Reading, writing, and modulating genetic information with boranophosphate mimics of nucleotides, DNA, and RNA, Annals of the New York Academy of Sciences 2003, 1002:12-29

114. Summers JS, Shaw BR: Boranophosphates as mimics of natural phosphodiesters in DNA, Current Medicinal Chemistry 2001, 8:1147-1155

115. Hall IH, Burnham BS, Rajendran KG, Chen SY, Sood A, Spielvogel BF, Shaw BR: Hypolipidemic activity of boronated nucleosides and nucleotides in rodents, Biomedicine and Pharmacotherapy 1993, 47:79-87

116. Hall AH, Wan J, Shaughnessy EE, Ramsay Shaw B, Alexander KA: RNA interference using boranophosphate siRNAs: Structure-activity relationships, Nucleic Acids Research 2004, 32:5991-6000

117. Kurreck J, Wyszko E, Gillen C, Erdmann VA: Design of antisense oligonucleotides stabilized by locked nucleic acids, Nucleic Acids Research 2002, 30:1911-1918 
118. Allerson CR, Sioufi N, Jarres R, Prakash TP, Naik N, Berdeja A, Wanders L, Griffey RH, Swayze EE, Bhat B: Fully 2'-modified oligonucleotide duplexes with improved in vitro potency and stability compared to unmodified small interfering RNA, Journal of Medicinal Chemistry 2005, 48:901-904

119. Nishizaki T, Iwai S, Ohtsuka E, Nakamura H: Solution structure of an RNA: 2'-O-methylated RNA hybrid duplex containing an RNA.DNA hybrid segment at the center, Biochemistry 1997, 36:2577-2585

120. Urban E, Noe CR: Structural modifications of antisense oligonucleotides, Farmaco 2003, 58:243-258

121. Sierakowska H, Sambade MJ, Agrawal S, Kole R: Repair of thalassemic human beta-globin mRNA in mammalian cells by antisense oligonucleotides, Proceedings of the National Academy of Sciences of the United States of America 1996, 93:12840-12844

122. Layzer JM, McCaffrey AP, Tanner AK, Huang Z, Kay MA, Sullenger BA: In vivo activity of nuclease-resistant siRNAs, RNA 2004, 10:766-771

123. Wahlestedt C, Salmi P, Good L, Kela J, Johnsson T, Hokfelt T, Broberger C, Porreca F, Lai J, Ren K, Ossipov M, Koshkin A, Jakobsen N, Skouv J, Oerum H, Jacobsen MH, Wengel J: Potent and nontoxic antisense oligonucleotides containing locked nucleic acids, Proceedings of the National Academy of Sciences of the United States of America 2000, 97:5633-5638

124. Swayze EE, Siwkowski AM, Wancewicz EV, Migawa MT, Wyrzykiewicz TK, Hung G, Monia BP, Bennett CF: Antisense oligonucleotides containing locked nucleic acid improve potency but cause significant hepatotoxicity in animals, Nucleic Acids Research 2007, 35:687-700

125. Elmen J, Thonberg H, Ljungberg K, Frieden M, Westergaard M, Xu Y, Wahren B, Liang Z, Orum H, Koch T, Wahlestedt C: Locked nucleic acid (LNA) mediated improvements in siRNA stability and functionality, Nucleic Acids Research 2005, 33:439-447

126. Miyagishi M, Hayashi M, Taira K: Comparison of the suppressive effects of antisense oligonucleotides and siRNAs directed against the same targets in mammalian cells, Antisense and Nucleic Acid Drug Development 2003, 13:1-7

127. Semizarov D, Frost L, Sarthy A, Kroeger P, Halbert DN, Fesik SW: Specificity of short interfering RNA determined through gene expression signatures, Proceedings of the National Academy of Sciences of the United States of America 2003, 100:6347-6352 
128. Qiu S, Adema CM, Lane T: A computational study of off-target effects of RNA interference, Nucleic Acids Research 2005, 33:1834-1847

129. Davis S, Lollo B, Freier S, Esau C: Improved targeting of miRNA with antisense oligonucleotides, Nucleic Acids Research 2006, 34:2294-2304

130. Yu D, Kandimalla ER, Roskey A, Zhao Q, Chen L, Chen J, Agrawal S: Stereo-enriched phosphorothioate oligodeoxynucleotides: Synthesis, biophysical and biological properties, Bioorganic and Medicinal Chemistry 2000, 8:275-284

131. Robbins M, Judge A, MacLachlan I: siRNA and innate immunity, Oligonucleotides 2009, 19:89-102

132. Cekaite L, Furset G, Hovig E, Sioud M: Gene expression analysis in blood cells in response to unmodified and 2'-modified siRNAs reveals TLR-dependent and independent effects, Journal of Molecular Biology 2007, 365:90-108

133. Takakura Y, Mahato RI, Hashida M: Extravasation of macromolecules, Advanced Drug Delivery Reviews 1998, 34:93-108

134. Seymour LW: Passive tumor targeting of soluble macromolecules and drug conjugates, Critical Reviews in Therapeutic Drug Carrier Systems 1992, 9:135187

135. Karnovsky MJ: The ultrastructural basis of capillary permeability studied with peroxidase as a tracer, The Journal of Cell Biology 1967, 35:213-236

136. Schnittler HJ, Wilke A, Gress T, Suttorp N, Drenckhahn D: Role of actin and myosin in the control of paracellular permeability in pig, rat and human vascular endothelium, The Journal of Physiology 1990, 431:379-401

137. Brett J, Gerlach H, Nawroth P, Steinberg S, Godman G, Stern D: Tumor necrosis factor/cachectin increases permeability of endothelial cell monolayers by a mechanism involving regulatory G proteins, The Journal of Experimental Medicine 1989, 169:1977-1991

138. Fang J, Seki T, Maeda H: Therapeutic strategies by modulating oxygen stress in cancer and inflammation, Advanced Drug Delivery Reviews 2009, 61:290-302

139. Cheng K, Ye Z, Guntaka RV, Mahato RI: Biodistribution and hepatic uptake of triplex-forming oligonucleotides against type alpha1(I) collagen gene promoter in normal and fibrotic rats, Molecular Pharmaceutics 2005, 2:206-217

140. Lebedeva I, Benimetskaya L, Stein CA, Vilenchik M: Cellular delivery of antisense oligonucleotides, European Journal of Pharmaceutics and Biopharmaceutics 2000, 50:101-119 
141. Farhood H, Serbina N, Huang L: The role of dioleoyl phosphatidylethanolamine in cationic liposome mediated gene transfer, Biochimica et Biophysica Acta 1995, 1235:289-295

142. Xu Y, Szoka FC, Jr.: Mechanism of DNA release from cationic liposome/DNA complexes used in cell transfection, Biochemistry 1996, 35:5616-5623

143. Boussif O, Lezoualch F, Zanta MA, Mergny MD, Scherman D, Demeneix B, Behr JP: A versatile vector for gene and oligonucleotide transfer into cells in culture and in vivo: Polyethylenimine, Proceedings of the National Academy of Sciences of the United States of America 1995, 92:7297-7301

144. Macara IG: Transport into and out of the nucleus, Microbiology and Molecular Biology Reviews 2001, 65:570-594

145. Felgner PL, Gadek TR, Holm M, Roman R, Chan HW, Wenz M, Northrop JP, Ringold GM, Danielsen M: Lipofection: A highly efficient, lipid-mediated DNA transfection procedure, Proceedings of the National Academy of Sciences of the United States of America 1987, 84:7413-7417

146. Zimmermann TS, Lee AC, Akinc A, Bramlage B, Bumcrot D, Fedoruk MN, Harborth J, Heyes JA, Jeffs LB, John M, Judge AD, Lam K, McClintock K, Nechev LV, Palmer LR, Racie T, Rohl I, Seiffert S, Shanmugam S, Sood V, Soutschek J, Toudjarska I, Wheat AJ, Yaworski E, Zedalis W, Koteliansky V, Manoharan M, Vornlocher HP, MacLachlan I: RNAi-mediated gene silencing in non-human primates, Nature 2006, 441:111-114

147. Zhu L, Lu Y, Miller DD, Mahato RI: Structural and formulation factors influencing pyridinium lipid-based gene transfer, Bioconjugate Chemistry 2008, 19:2499-2512

148. Morille M, Passirani C, Vonarbourg A, Clavreul A, Benoit JP: Progress in developing cationic vectors for non-viral systemic gene therapy against cancer, Biomaterials 2008, 29:3477-3496

149. Sato Y, Murase K, Kato J, Kobune M, Sato T, Kawano Y, Takimoto R, Takada K, Miyanishi K, Matsunaga T, Takayama T, Niitsu Y: Resolution of liver cirrhosis using vitamin A-coupled liposomes to deliver siRNA against a collagen-specific chaperone, Nature Biotechnology 2008, 26:431-442

150. Akinc A, Zumbuehl A, Goldberg M, Leshchiner ES, Busini V, Hossain N, Bacallado SA, Nguyen DN, Fuller J, Alvarez R, et al.: A combinatorial library of lipid-like materials for delivery of RNAi therapeutics, Nature Biotechnology 2008, 26:561-569 
151. Godbey WT, Wu KK, Mikos AG: Poly(ethylenimine) and its role in gene delivery, Journal of Controlled Release 1999, 60:149-160

152. Wagner E, Ogris M, Zauner W: Polylysine-based transfection systems utilizing receptor-mediated delivery, Advanced Drug Delivery Reviews 1998, 30:97-113

153. Haensler J, Szoka FC, Jr.: Polyamidoamine cascade polymers mediate efficient transfection of cells in culture, Bioconjugate Chemistry 1993, 4:372-379

154. van de Wetering P, Moret EE, Schuurmans-Nieuwenbroek NM, van Steenbergen MJ, Hennink WE: Structure-activity relationships of water-soluble cationic methacrylate/methacrylamide polymers for nonviral gene delivery, Bioconjugate Chemistry 1999, 10:589-597

155. Fischer D, Bieber T, Li Y, Elsasser HP, Kissel T: A novel non-viral vector for DNA delivery based on low molecular weight, branched polyethylenimine: Effect of molecular weight on transfection efficiency and cytotoxicity, Pharmaceutical Research 1999, 16:1273-1279

156. Fischer D, Li Y, Ahlemeyer B, Krieglstein J, Kissel T: In vitro cytotoxicity testing of polycations: Influence of polymer structure on cell viability and hemolysis, Biomaterials 2003, 24:1121-1131

157. Funhoff AM, van Nostrum CF, Koning GA, Schuurmans-Nieuwenbroek NM, Crommelin DJ, Hennink WE: Endosomal escape of polymeric gene delivery complexes is not always enhanced by polymers buffering at low $\mathrm{pH}$, Biomacromolecules 2004, 5:32-39

158. Godbey WT, Barry MA, Saggau P, Wu KK, Mikos AG:

Poly(ethylenimine)-mediated transfection: A new paradigm for gene delivery, Journal of Biomedical Materials Research 2000, 51:321-328

159. Forrest ML, Koerber JT, Pack DW: A degradable polyethylenimine derivative with low toxicity for highly efficient gene delivery, Bioconjugate Chemistry 2003, 14:934-940

160. Petersen H, Fechner PM, Martin AL, Kunath K, Stolnik S, Roberts CJ, Fischer D, Davies MC, Kissel T: Polyethylenimine-graft-poly(ethylene glycol) copolymers: Influence of copolymer block structure on DNA complexation and biological activities as gene delivery system, Bioconjugate Chemistry 2002, 13:845-854

161. Vinogradov SV, Bronich TK, Kabanov AV: Self-assembly of polyamine-poly(ethylene glycol) copolymers with phosphorothioate oligonucleotides, Bioconjugate Chemistry 1998, 9:805-812 
162. Leclercq F, Dubertret C, Pitard B, Scherman D, Herscovici J: Synthesis of glycosylated polyethylenimine with reduced toxicity and high transfecting efficiency, Bioorganic and Medicinal Chemistry Letters 2000, 10:1233-1235

163. Bosman AW, Janssen HM, Meijer EW: About dendrimers: Structure, physical properties, and applications, Chemical Reviews 1999, 99:1665-1688

164. Bielinska A, Kukowska-Latallo JF, Johnson J, Tomalia DA, Baker JR, Jr.: Regulation of in vitro gene expression using antisense oligonucleotides or antisense expression plasmids transfected using starburst PAMAM dendrimers, Nucleic Acids Research 1996, 24:2176-2182

165. Zhou J, Wu J, Hafdi N, Behr JP, Erbacher P, Peng L: PAMAM dendrimers for efficient siRNA delivery and potent gene silencing, Chemical Communications 2006, 2362-2364

166. Rajur SB, Roth CM, Morgan JR, Yarmush ML: Covalent protein-oligonucleotide conjugates for efficient delivery of antisense molecules, Bioconjugate Chemistry 1997, 8:935-940

167. Maier MA, Yannopoulos CG, Mohamed N, Roland A, Fritz H, Mohan V, Just G, Manoharan M: Synthesis of antisense oligonucleotides conjugated to a multivalent carbohydrate cluster for cellular targeting, Bioconjugate Chemistry 2003, 14:18-29

168. Jeong JH, Mok H, Oh YK, Park TG: siRNA conjugate delivery systems, Bioconjugate Chemistry 2008, 20:5-14

169. Ye Z, Cheng K, Guntaka RV, Mahato RI: Targeted delivery of a triplex-forming oligonucleotide to hepatic stellate cells, Biochemistry 2005, 44:4466-4476

170. Ye Z, Cheng K, Guntaka RV, Mahato RI: Receptor-mediated hepatic uptake of M6P-BSA-conjugated triplex-forming oligonucleotides in rats, Bioconjugate Chemistry 2006, 17:823-830

171. Kopecek J, Kopeckova P, Minko T, Lu Z: HPMA copolymer-anticancer drug conjugates: Design, activity, and mechanism of action, European Journal of Pharmaceutics and Biopharmaceutics 2000, 50:61-81

172. Yang N, Ye Z, Li F, Mahato RI: HPMA polymer-based site-specific delivery of oligonucleotides to hepatic stellate cells, Bioconjugate Chemistry 2009, 20:213221

173. Zhao H, Greenwald RB, Reddy P, Xia J, Peng P: A new platform for oligonucleotide delivery utilizing the PEG prodrug approach, Bioconjugate Chemistry 2005, 16:758-766 
174. Kim SH, Jeong JH, Lee SH, Kim SW, Park TG: PEG conjugated VEGF siRNA for anti-angiogenic gene therapy, Journal of Controlled Release 2006, 116:123129

175. Zhu L, Ye Z, Cheng K, Miller DD, Mahato RI: Site-specific delivery of oligonucleotides to hepatocytes after systemic administration, Bioconjugate Chemistry 2008, 19:290-298

176. Soutschek J, Akinc A, Bramlage B, Charisse K, Constien R, Donoghue M, Elbashir S, Geick A, Hadwiger P, Harborth J, John M, Kesavan V, Lavine G, Pandey RK, Racie T, Rajeev KG, Rohl I, Toudjarska I, Wang G, Wuschko S, Bumcrot D, Koteliansky V, Limmer S, Manoharan M, Vornlocher HP: Therapeutic silencing of an endogenous gene by systemic administration of modified siRNAs, Nature 2004, 432:173-178

177. Cheng K, Ye Z, Guntaka RV, Mahato RI: Enhanced hepatic uptake and bioactivity of type alpha1(I) collagen gene promoter-specific triplex-forming oligonucleotides after conjugation with cholesterol, Journal of Pharmacology and Experimental Therapeutics 2006, 317:797-805

178. Lorenz C, Hadwiger P, John M, Vornlocher HP, Unverzagt C: Steroid and lipid conjugates of siRNAs to enhance cellular uptake and gene silencing in liver cells, Bioorganic and Medicinal Chemistry Letters 2004, 14:4975-4977

179. Nishina K, Unno T, Uno Y, Kubodera T, Kanouchi T, Mizusawa H, Yokota T: Efficient in vivo delivery of siRNA to the liver by conjugation of alphatocopherol, Molecular Therapy 2008, 16:734-740

180. Agrawal S, Temsamani J, Tang JY: Pharmacokinetics, biodistribution, and stability of oligodeoxynucleotide phosphorothioates in mice, Proceedings of the National Academy of Sciences of the United States of America 1991, 88:75957599

181. Cossum PA, Sasmor H, Dellinger D, Truong L, Cummins L, Owens SR, Markham PM, Shea JP, Crooke S: Disposition of the 14C-labeled phosphorothioate oligonucleotide ISIS 2105 after intravenous administration to rats, Journal of Pharmacology and Experimental Therapeutics 1993, 267:11811190

182. Geary RS, Watanabe TA, Truong L, Freier S, Lesnik EA, Sioufi NB, Sasmor H, Manoharan M, Levin AA: Pharmacokinetic properties of 2'-O-(2-methoxyethyl) modified oligonucleotide analogs in rats, Journal of Pharmacology and Experimental Therapeutics 2001, 296:890-897 
183. Zhang R, Diasio RB, Lu Z, Liu T, Jiang Z, Galbraith WM, Agrawal S: Pharmacokinetics and tissue distribution in rats of an oligodeoxynucleotide phosphorothioate (GEM 91) developed as a therapeutic agent for human immunodeficiency virus type-1, Biochemical Pharmacology 1995, 49:929-939

184. Sewell KL, Geary RS, Baker BF, Glover JM, Mant TG, Yu RZ, Tami JA, Dorr FA: Phase I trial of ISIS 104838, a 2'-methoxyethyl modified antisense oligonucleotide targeting tumor necrosis factor-alpha, Journal of Pharmacology and Experimental Therapeutics 2002, 303:1334-1343

185. Yu RZ, Kim TW, Hong A, Watanabe TA, Gaus HJ, Geary RS: Cross-species pharmacokinetic comparison from mouse to man of a second-generation antisense oligonucleotide, ISIS 301012, targeting human apolipoprotein B-100, Drug Metabolism and Disposition 2007, 35:460-468

186. Fluiter K, ten Asbroek AL, de Wissel MB, Jakobs ME, Wissenbach M, Olsson H, Olsen $\mathrm{O}$, Oerum H, Baas F: In vivo tumor growth inhibition and biodistribution studies of locked nucleic acid (LNA) antisense oligonucleotides, Nucleic Acids Research 2003, 31:953-962

187. Yang CJ, Wang L, Wu Y, Kim Y, Medley CD, Lin H, Tan W: Synthesis and investigation of deoxyribonucleic acid/locked nucleic acid chimeric molecular beacons, Nucleic Acids Research 2007, 35:4030-4041

188. McMahon BM, Mays D, Lipsky J, Stewart JA, Fauq A, Richelson E: Pharmacokinetics and tissue distribution of a peptide nucleic acid after intravenous administration, Antisense and Nucleic Acid Drug Development 2002, $12: 65-70$

189. Braasch DA, Paroo Z, Constantinescu A, Ren G, Oz OK, Mason RP, Corey DR: Biodistribution of phosphodiester and phosphorothioate siRNA, Bioorganic and Medicinal Chemistry Letters 2004, 14:1139-1143

190. van de Water FM, Boerman OC, Wouterse AC, Peters JG, Russel FG, Masereeuw R: Intravenously administered short interfering RNA accumulates in the kidney and selectively suppresses gene function in renal proximal tubules, Drug Metabolism and Disposition 2006, 34:1393-1397

191. Wolfrum C, Shi S, Jayaprakash KN, Jayaraman M, Wang G, Pandey RK, Rajeev KG, Nakayama T, Charrise K, Ndungo EM: Mechanisms and optimization of in vivo delivery of lipophilic siRNAs, Nature Biotechnology 2007, 25:1149-1157

192. Merkel OM, Librizzi D, Pfestroff A, Schurrat T, Behe M, Kissel T: In vivo SPECT and real-time gamma camera imaging of biodistribution and pharmacokinetics of siRNA delivery using an optimized radiolabeling and purification procedure, Bioconjugate Chemistry 2008, 20:174-182 
193. Roberts J, Palma E, Sazani P, Orum H, Cho M, Kole R: Efficient and persistent splice switching by systemically delivered LNA oligonucleotides in mice, Molecular Therapy 2006, 14:471-475

194. Crooke ST: Progress in antisense technology: The end of the beginning, Methods in Enzymology 2000, 313:3-45

195. Dove A: Isis and antisense face crucial test without Novartis, Nature Biotechnology 2000, 18:19

196. Kleinman ME, Yamada K, Takeda A, Chandrasekaran V, Nozaki M, Baffi JZ, Albuquerque RJ, Yamasaki S, Itaya M, Pan Y, Appukuttan B, Gibbs D, Yang Z, Kariko K, Ambati BK, Wilgus TA, DiPietro LA, Sakurai E, Zhang K, Smith JR, Taylor EW, Ambati J: Sequence- and target-independent angiogenesis suppression by siRNA via TLR3, Nature 2008, 452:591-597

197. Ye Z, Houssein HSH, Mahato RI: Bioconjugation of oligonucleotides for treating liver fibrosis, Oligonucleotides 2007, 17:349-404

198. Friedman SL: Molecular regulation of hepatic fibrosis, an integrated cellular response to tissue injury, Journal of Biological Chemistry 2000, 275:2247-2250

199. Pinzani M, Rombouts K, Colagrande S: Fibrosis in chronic liver diseases: Diagnosis and management, Journal of Hepatology 2005, 42:S22-S36

200. Hagens WI, Mattos A, Greupink R, de Jager-Krikken A, Reker-Smit C, van Loenen-Weemaes AM, Gouw ASH, Poelstra K, Beljaars L: Targeting 15d-prostaglandin J2 to hepatic stellate cells: Two options evaluated, Pharmaceutical Research 2007, 24:566-574

201. Cheng K, Mahato RI: Gene modulation for treating liver fibrosis, Critical Reviews in Therapeutic Drug Carrier Systems 2007, 24:93-146

202. Sawai K, Mahato RI, Oka Y, Takakura Y, Hashida M: Disposition of oligonucleotides in isolated perfused rat kidney: Involvement of scavenger receptors in their renal uptake, Journal of Pharmacology and Experimental Therapeutics 1996, 279:284-290

203. Agrawal S, Temsamani J, Tang JY: Pharmacokinetics, biodistribution, and stability of oligodeoxynucleotide phosphorothioates in mice, Proceedings of the National Academy of Sciences of the United States of America 1991, 88:75957599

204. Takakura Y, Mahato RI, Yoshida M, Kanamaru T, Hashida M: Uptake characteristics of oligonucleotides in the isolated rat liver perfusion system, Antisense and Nucleic Acid Drug Development 1996, 6:177-183 
205. Kopeek J, Kopekova P, Minko T, Lu ZR: HPMA copolymer-anticancer drug conjugates: Design, activity, and mechanism of action, European Journal of Pharmaceutics and Biopharmaceutics 2000, 50:61-81

206. Gao SQ, Lu ZR, Petri B, Kopeckova P, Kopecek J: Colon-specific 9-aminocamptothecin-HPMA copolymer conjugates containing a 1, 6-elimination spacer, Journal of Controlled Release 2006, 110:323-331

207. Sirova M, Strohalm J, Subr V, Plocova D, Rossmann P, Mrkvan T, Ulbrich K, Rihova B: Treatment with HPMA copolymer-based doxorubicin conjugate containing human immunoglobulin induces long-lasting systemic anti-tumour immunity in mice, Cancer Immunology, Immunotherapy 2007, 56:35-47

208. Jensen KD, Kopeckova P, Kopecek J: Antisense oligonucleotides delivered to the lysosome escape and actively inhibit the hepatitis B virus, Bioconjugate Chemistry 2002, 13:975-984

209. Jensen KD, Nori A, Tijerina M, Kopeckova P, Kopecek J: Cytoplasmic delivery and nuclear targeting of synthetic macromolecules, Journal of Controlled Release 2003, 87:89-105

210. Wang L, Kristensen J, Ruffner DE: Delivery of antisense oligonucleotides using HPMA polymer: Synthesis of A thiol polymer and its conjugation to watersoluble molecules, Bioconjugate Chemistry 1998, 9:749-757

211. Monsigny M, Petit C, Roche AC: Colorimetric determination of neutral sugars by a resorcinol sulfuric acid micromethod, Analytical Biochemistry 1988, 175:525530

212. Lu ZR, Gao SQ, Kopeckova P, Kopecek J: Modification of cyclosporin A and conjugation of its derivative to HPMA copolymers, Bioconjugate Chemistry 2001, 12:129-133

213. Ding H, Kopeckova P, Kopecek J: Self-association properties of HPMA copolymers containing an amphipathic heptapeptide, Journal of Drug Targeting 2007, 15:465-474

214. De Bleser PJ, Scott CD, Niki T, Xu G, Wisse E, Geerts A: Insulin like growth factor II/mannose 6 phosphate receptor expression in liver and serum during acute CCl4 intoxication in the rat, Hepatology 1996, 23:1530-1537

215. Beljaars L, Olinga P, Molema G, De Bleser P, Geerts A, Groothuis GMM, Meijer DKF, Poelstra K: Characteristics of the hepatic stellate cell selective carrier mannose 6 phosphate modified albumin (M6P28 HSA), Liver 2001, 21:320-328 
216. Beljaars L, Molema G, Weert B, Bonnema H, Olinga P, Groothuis GM, Meijer DK, Poelstra K: Albumin modified with mannose 6 phosphate: A potential carrier for selective delivery of antifibrotic drugs to rat and human hepatic stellate cells, Hepatology 1999, 29:1486-1493

217. Rajur S, Roth C, Morgan J, Yarmush M: Covalent protein-oligonucleotide conjugates for efficient delivery of antisense molecules, Bioconjugate Chemistry 1997, 8:935-940

218. Ye Z, Cheng K, Guntaka RV, Mahato RI: Receptor-mediated hepatic uptake of M6P-BSA-conjugated triplex-forming oligonucleotides in rats, Bioconjugate Chemistry 2006, 17:823-830

219. Rachmawati H, Reker-Smit C, Lub-de Hooge MN, van Loenen-Weemaes A, Poelstra K, Beljaars L: Chemical modification of interleukin-10 with mannose 6-phosphate groups yields a liver-selective cytokine, Drug Metabolism and Disposition 2007, 35:814-821

220. Bataller R, Brenner DA: Liver fibrosis, The Journal of Clinical Investigation 2005, 115:209-218

221. Lotersztajn S, Julien B, Teixeira-Clerc F, Grenard P, Mallat A: Hepatic fibrosis: Molecular mechanisms and drug targets, Annual Review of Pharmacology and Toxicology 2005, 45:605-628

222. Panakanti R, Pratap A, Yang N, Jackson JS, Mahato RI: Triplex forming oligonucleotides against type alpha 1 (I) collagen attenuates liver fibrosis induced by bile duct ligation, Biochemical Pharmacology 2010, 80:1718-1726

223. Saito JM, Maher JJ: Bile duct ligation in rats induces biliary expression of cytokine-induced neutrophil chemoattractant, Gastroenterology 2000, 118:11571168

224. Gujral JS, Farhood A, Bajt ML, Jaeschke H: Neutrophils aggravate acute liver injury during obstructive cholestasis in bile duct-ligated mice, Hepatology 2003, $38: 355-363$

225. Cooney M, Czernuszewicz G, Postel EH, Flint SJ, Hogan ME: Site-specific oligonucleotide binding represses transcription of the human c-myc gene in vitro, Science 1988, 241:456-459

226. Vasquez KM, Narayanan L, Glazer PM: Specific mutations induced by triplex-forming oligonucleotides in mice, Science 2000, 290:530-533 
227. Vasquez KM, Wang G, Havre PA, Glazer PM: Chromosomal mutations induced by triplex-forming oligonucleotides in mammalian cells, Nucleic Acids Research 1999, 27:1176-1181

228. Van Eyken P, Sciot R, Desmet V: A cytokeratin immunohistochemical study of cholestatic liver disease: Evidence that hepatocytes can express' bile duct-type' cytokeratins, Histopathology 1989, 15:125-135

229. Harlan JM: Leukocyte-endothelial interactions, Blood 1985, 65:513-525

230. Wright SD, Detmers PA: Adhesion-promoting receptors on phagocytes, Journal of Cell Science Supplement 1988, 9:99-120

231. McEver RP: Leukocyte-endothelial cell interactions, Current Opinion in Cell Biology 1992, 4:840-849

232. Bevilacqua MP: Endothelial-leukocyte adhesion molecules, Annual Review of Immunology 1993, 11:767-804

233. Springer TA: Adhesion receptors of the immune system, Nature 1990, 346:425434

234. Mahato RI, Cheng K, Guntaka RV: Modulation of gene expression by antisense and antigene oligodeoxynucleotides and small interfering RNA, Expert Opinion on Drug Delivery 2005, 2:3-28

235. Iredale JP: Cirrhosis: New research provides a basis for rational and targeted treatments, The British Medical Journal 2003, 327:143-147

236. Bauer M, Schuppan D: TGF-beta1 in liver fibrosis: Time to change paradigms, FEBS Letters 2001, 502:1-3

237. Shek FW, Benyon RC: How can transforming growth factor beta be targeted usefully to combat liver fibrosis, European Journal of Gastroenterology and Hepatology 2004, 16:123-126

238. Knittel T, Mehde M, Kobold D, Saile B, Dinter C, Ramadori G: Expression patterns of matrix metalloproteinases and their inhibitors in parenchymal and non-parenchymal cells of rat liver: Regulation by TNF-alpha and TGF-beta1, Journal of Hepatology 1999, 30:48-60

239. Friedman SL: Liver fibrosis-from bench to bedside, Journal of Hepatology 2003, 38:S38-S53 
240. Qi Z, Atsuchi N, Ooshima A, Takeshita A, Ueno H: Blockade of type transforming growth factor signaling prevents liver fibrosis and dysfunction in the rat, Proceedings of the National Academy of Sciences of the United States of America 1999, 96:2345-2349

241. Gouville AC, Boullay V, Krysa G, Pilot J, Brusq JM, Loriolle F, Gauthier JM, Papworth SA, Laroze A, Gellibert F: Inhibition of TGF signaling by an ALK5 inhibitor protects rats from dimethylnitrosamine induced liver fibrosis, British Journal of Pharmacology 2005, 145:166-177

242. George J, Roulot D, Koteliansky VE, Bissell DM: In vivo inhibition of rat stellate cell activation by soluble transforming growth factor type II receptor: A potential new therapy for hepatic fibrosis, Proceedings of the National Academy of Sciences of the United States of America 1999, 96:12719-12724

243. Okuno M, Akita K, Moriwaki H, Kawada N, Ikeda K, Kaneda K, Suzuki Y, Kojima S: Prevention of rat hepatic fibrosis by the protease inhibitor, camostat mesilate, via reduced generation of active TGF-beta, Gastroenterology 2001, 120:1784-1800

244. Arias E, Anderson RN, Kung HC, Murphy SL, Kochanek KD: Deaths: Final data for 2001, National Vital Statistics Reports 2003, 52:1-116

245. Li G, Shi Y, Li D, Xie Q, Guo Q, Jin Y: Effect of small interfering RNA targeting connective tissue growth factor on the synthesis and secretion of extracellular matrix in hepatic stellate cells, Chinese Journal of Hepatology 2004, 12:526-529

246. Zhou X, Murphy FR, Gehdu N, Zhang J, Iredale JP, Benyon RC: Engagement of v 3 integrin regulates proliferation and apoptosis of hepatic stellate cells, Journal of Biological Chemistry 2004, 279:23996-24006

247. Lindquist JN, Parsons CJ, Stefanovic B, Brenner DA: Regulation of 1 (I) collagen messenger RNA decay by interactions with $\mathrm{CP}$ at the 3 '-untranslated region, Journal of Biological Chemistry 2004, 279:23822-23829

248. Song E, Lee SK, Wang J, Ince N, Ouyang N, Min J, Chen J, Shankar P, Lieberman J: RNA interference targeting Fas protects mice from fulminant hepatitis, Nature Medicine 2003, 9:347-351

249. Kushibiki T, Nagata-Nakajima N, Sugai M, Shimizu A, Tabata Y: Delivery of plasmid DNA expressing small interference RNA for TGF-beta type II receptor by cationized gelatin to prevent interstitial renal fibrosis, Journal of Controlled Release 2005, 105:318-331

250. Pfaffl MW: A new mathematical model for relative quantification in real-time RT-PCR, Nucleic Acids Research 2001, 29:e45 
251. Liu X, Hu H, Yin JQ: Therapeutic strategies against TGF signaling pathway in hepatic fibrosis, Liver International 2006, 26:8-22

252. Hellerbrand C, Stefanovic B, Giordano F, Burchardt ER, Brenner DA: The role of TGF-beta1 in initiating hepatic stellate cell activation in vivo, Journal of Hepatology 1999, 30:77-87

253. Chen Y, Mahato RI: siRNA pool targeting different sites of human hepatitis B surface antigen efficiently inhibits HBV infection, Journal of Drug Targeting 2008, 16:140-148

254. Malizia G, Brunt EM, Peters MG, Rizzo A, Broekelmann TJ, McDonald JA: Growth factor and procollagen type I gene expression in human liver disease, Gastroenterology 1995, 108:145-156

255. Jonsson JR, Barrie HD, O'Rourke P, Clouston AD, Powell EE: Obesity and steatosis influence serum and hepatic inflammatory markers in chronic hepatitis C, Hepatology 2008, 48:80-87

256. Tipoe GL, Liong EC, Casey CA, Donohue Jr TM, Eagon PK, So H, Leung TM, Fogt F, Nanji AA: A voluntary oral ethanol feeding rat model associated with necroinflammatory liver injury, Alcoholism: Clinical and Experimental Research 2008, 32:669-682

257. Bortolami M, Kotsafti A, Cardin R, Farinati F: Fas/FasL system, IL-1 expression and apoptosis in chronic HBV and HCV liver disease, Journal of Viral Hepatitis 2008, 15:515-522

258. Friedman SL: Mechanisms of hepatic fibrogenesis, Gastroenterology 2008, 134:1655-1669

259. Cutroneo KR: TGF-induced fibrosis and SMAD signaling: Oligo decoys as natural therapeutics for inhibition of tissue fibrosis and scarring, Wound Repair and Regeneration 2007, 15:S54-S60

260. De Paula D, Bentley M, Mahato RI: Hydrophobization and bioconjugation for enhanced siRNA delivery and targeting, RNA 2007, 13:431-456

261. Rumi M, Ishihara S, Aziz M, Kazumori H, Ishimura N, Yuki T, Kadota C, Kadowaki Y, Kinoshita Y: RNA polymerase II mediated transcription from the polymerase III promoters in short hairpin RNA expression vector, Biochemical and Biophysical Research Communications 2006, 339:540-547 
262. Liu Y, Wen XM, Lui ELH, Friedman SL, Cui W, Ho NPS, Li L, Ye T, Fan ST, Zhang H: Therapeutic targeting of the PDGF and TGF-signaling pathways in hepatic stellate cells by PTK787/ZK22258, Laboratory Investigation 2009, $89: 1152-1160$

263. Doh KO, Jung HK, Moon IJ, Kang HG, Park JH, Park JG: Prevention of CCl4-induced liver cirrhosis by ribbon antisense to transforming growth factor-B1, International Journal of Molecular Medicine 2008, 21:33-39

264. Cassiman D, Libbrecht L, Desmet V, Denef C, Roskams T: Hepatic stellate cell/myofibroblast subpopulations in fibrotic human and rat livers, Journal of Hepatology 2002, 36:200-209

265. Chen S, Chen Y, Zhang X, Qian H, Chen W, Xie W: Targeted inhibition of platelet-derived growth factor receptor subunit in hepatic stellate cells ameliorates hepatic fibrosis in rats, Gene Therapy 2008, 15:1424-1435

266. Maubach G, Lim MCC, Zhang CY, Zhuo L: GFAP promoter directs lacZ expression specifically in a rat hepatic stellate cell line, World Journal of Gastroenterology 2006, 12:723-730

267. Ji J, Zhang J, Huang G, Qian J, Wang X, Mei S: Over-expressed microRNA-27a and $27 \mathrm{~b}$ influence fat accumulation and cell proliferation during rat hepatic stellate cell activation, FEBS Letters 2009, 583:759-766

268. Boudreau RL, Monteys AM, Davidson BL: Minimizing variables among hairpin based RNAi vectors reveals the potency of shRNAs, RNA 2008, 14:1834-1844

269. Li L, Lin X, Khvorova A, Fesik SW, Shen Y: Defining the optimal parameters for hairpin-based knockdown constructs, RNA 2007, 13:1765-1774

270. Lee Y, Ahn C, Han J, Choi H, Kim J, Yim J, Lee J, Provost P, Rådmark O, Kim S: The nuclear RNase III Drosha initiates microRNA processing, Nature 2003, 425:415-419

271. Chen CZ, Li L, Lodish HF, Bartel DP: MicroRNAs modulate hematopoietic lineage differentiation, Science 2004, 303:83-86

272. Han J, Lee Y, Yeom KH, Nam JW, Heo I, Rhee JK, Sohn SY, Cho Y, Zhang BT, Kim VN: Molecular basis for the recognition of primary microRNAs by the Drosha-DGCR8 complex, Cell 2006, 125:887-901

273. Chen G, Khalil N: TGF-beta1 increases proliferation of airway smooth muscle cells by phosphorylation of map kinases, Respiratory Research 2006, 7:2 
274. Wu S, Theodorescu D, Kerbel RS, Willson J, Mulder KM, Humphrey LE, Brattain MG: TGF-beta1 is an autocrine-negative growth regulator of human colon carcinoma FET cells in vivo as revealed by transfection of an antisense expression vector, The Journal of Cell Biology 1992, 116:187-196

275. Yan Z, Kim GY, Deng X, Friedman E: Transforming growth factor 1 induces proliferation in colon carcinoma cells by Ras-dependent, smad-independent down-regulation of p21cip1, Journal of Biological Chemistry 2002, 277:98709879

276. Sun T, Adra S, Smallwood R, Holcombe M, MacNeil S: Exploring hypotheses of the actions of TGF-beta1 in epidermal wound healing using a 3D computational multiscale model of the human epidermis, Plos One 2009, 4:e8515

277. Purps O, Lahme B, Gressner AM, Meindl-Beinker NM, Dooley S: Loss of TGF beta dependent growth control during HSC transdifferentiation, Biochemical and Biophysical Research Communications 2007, 353:841-847

278. Lee MK, Pardoux C, Hall MC, Lee PS, Warburton D, Qing J, Smith SM, Derynck R: TGF-beta activates Erk MAP kinase signalling through direct phosphorylation of ShcA, The EMBO Journal 2007, 26:3957-3967

279. Saile B, Matthes N, El Armouche H, Neubauer K, Ramadori G: The bcl, NF kappa B and p53/p21WAF1 systems are involved in spontaneous apoptosis and in the anti-apoptotic effect of TGF-beta or TNF-alpha on activated hepatic stellate cells, European Journal of Cell Biology 2001, 80:554-561

280. Maher T: Pirfenidone in idiopathic pulmonary fibrosis, Drugs of Today 2010, $46: 473-482$

281. Zhao XY, Zeng X, Li XM, Wang TL, Wang BE: Pirfenidone inhibits carbon tetrachloride and albumin complex induced liver fibrosis in rodents by preventing activation of hepatic stellate cells, Clinical and Experimental Pharmacology and Physiology 2009, 36:963-968

282. Hecht JH, Weiner JA, Post SR, Chun J: Ventricular zone gene-1 (vzg-1) encodes a lysophosphatidic acid receptor expressed in neurogenic regions of the developing cerebral cortex, The Journal of Cell Biology 1996, 135:1071-1083

283. Chun J, Goetzl EJ, Hla T, Igarashi Y, Lynch KR, Moolenaar W, Pyne S, Tigyi G: International union of pharmacology. XXXIV. Lysophospholipid receptor nomenclature, Pharmacological Reviews 2002, 54:265-269

284. Noguchi K, Herr D, Mutoh T, Chun J: Lysophosphatidic acid (LPA) and its receptors, Current Opinion in Pharmacology 2009, 9:15-23 
285. Li F, Lu Y, Li W, Miller DD, Mahato RI: Synthesis, formulation and in vitro evaluation of a novel microtubule destabilizer, SMART-100, Journal of Controlled Release 2010, 143:151-158

286. Rancoule C, Pradere JP, Gonzalez J, Klein J, Valet P, Bascands JL, Schanstra JP, Saulnier-Blache JS: Lysophosphatidic acid-1-receptor targeting agents for fibrosis, Expert Opinion on Investigational Drugs 2011, 1-11

287. Zhu J, Wu J, Frizell E, Liu SL, Bashey R, Rubin R, Norton P, Zern MA: Rapamycin inhibits hepatic stellate cell proliferation in vitro and limits fibrogenesis in an in vivo model of liver fibrosis, Gastroenterology 1999, 117:1198-1204

288. Otsuka H, Nagasaki Y, Kataoka K: PEGylated nanoparticles for biological and pharmaceutical applications, Advanced Drug Delivery Reviews 2003, 55:403-419 


\section{VITA}

Ningning Yang was born in Lianshui, Jiangsu, China, in 1977 and grew up in Nanjing, Jiangsu, China until she obtained her Masters degree. She joined Nanjing University and obtained her Bachelors degree in AstroPhysics in 1999 and Masters degree in Physiology in 2003. In the fall of 2006, she joined the graduate program in the Department of Pharmaceutical Sciences, the University of Tennessee Health Science Center, Memphis, from where she will receive her Ph.D. in Pharmaceutics (2011) under the supervision of Prof. Ram I. Mahato.

\section{HONORS and AWARDS}

1. Best poster award. Treatment of liver fibrosis after systemic administration of M6P-HPMA-TFO. The 15th International Symposium on Recent Advances in Drug Delivery Systems. (Salt Lake City, UT, 2011)

2. Selected podium presentation. M6P assisted site-specific delivery of oligonucleotides to hepatic stellate cells. The 35th Controlled Release Society Annual Meeting \& Exposition. (New York , NY, 2008)

\section{CERTIFICATE}

Base Programming for SAS 9

\section{ORIGINAL ARTICLES}

1. Pratap A, Pannakanti R, Yang N, Eason JD, Mahato RI: Cyclopamine attenuates acute warm ischemia reperfusion injury in cholestatic rat liver: Hope for marginal livers, Molecular Pharmaceutics 2011, 8:958-968

2. Yang N, Mahato RI: GFAP promoter driving RNA interference on TGF- $\beta 1$ to treat liver fibrosis, Pharmaceutical Research 2011, 28:752-761

3. Panakanti R, Pratap A, Yang N, Jackson JS, Mahato RI: Triplex forming oligonucleotides against type $\alpha 1$ (I) collagen attenuates liver fibrosis induced by bile duct ligation, Biochemical Pharmacology 2010, 80:1718-1726

4. Pratap A, Panakanti R, Yang N, Eason JD, Mahato RI: Inhibition of endogenous hedgehog signaling protects against acute liver injury after ischemia reperfusion, Pharmaceutical Research 2010, 27:2492-2504 
5. Cheng K, Yang $N^{*}$, Mahato RI: TGF- $\beta 1$ gene silencing for treating liver fibrosis, Molecular Pharmaceutics 2009, 6:772-779 (*co-first author)

6. Yang N, Ye Z, Li F, Mahato RI: HPMA Polymer-based site-specific delivery of oligonucleotides to hepatic stellate cells, Bioconjugate Chemistry 2009, 20:213221

7. Yang N*, Hou Y: The physiological significance of rabbit directionally selective retinal ganglion cells branching pattern properties, Chinese Journal of Neuroanatomy 2004, 20:329-336 (*corresponding author)

\section{BOOK CHAPTER}

Yang N, Mahato RI: Delivery and targeting of oligonucleotides and siRNA. In Narang AS and Mahato RI editors: Targeted Delivery of Small and Macromolecular Drugs, Boca Raton, FL: CRC Press, 2009, pp. 147-169

\section{PRESENTATIONS}

1. Yang N, Panakanti R and Mahato RI (Post). Treatment of liver fibrosis after systemic administration of M6P-HPMA-TFO. (The 15th International Symposium on Recent Advances in Drug Delivery Systems, Salt Lake City, Utah, 2011)

2. Yang $\mathrm{N}$ and Mahato RI (Post). Hepatic stellate cell-specific TGF- $\beta 1$ gene silencing for treating liver fibrosis. (ASGCT 13th Annual Meeting, Washington, DC, 2010)

3. Yang N (Podium). M6P assisted site-specific delivery of oligonucleotides to hepatic stellate cells. (The 35th Controlled Release Society Annual Meeting \& Exposition, New York, NY, 2008) 